
T. E. Venkata Balaji

An Introduction to Families, Deformations and Moduli

This work is licensed under the Creative Commons License 3.0 "by-nd", allowing you to download, distribute and print the document in a few copies for private or educational use, given that the document stays unchanged and the creator is mentioned.

You are not allowed to sell copies of the free version.

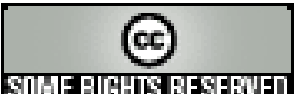

SORERIGHIS RESERVED 
erschienen in der Reihe der Universitätsdrucke im Universitätsverlag Göttingen 2010 
T. E. Venkata Balaji

\section{An Introduction to Families, Deformations and Moduli}

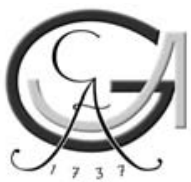

Universitätsverlag Göttingen 2010 
Bibliographische Information der Deutschen Nationalbibliothek

Die Deutsche Nationalbibliothek verzeichnet diese Publikation in der Deutschen Nationalbibliographie; detaillierte bibliographische Daten sind im Internet über $<$ http://dnb.ddb.de> abrufbar.

\author{
Address of the Author \\ T. E. Venkata Balaji \\ Department of Mathematics \\ Indian Institute of Technology Madras \\ Chennai 600036, India. \\ e-mail: tevbal@iitm.ac.in
}

This work is protected by German Intellectual Property Right Law.

It is also available as an Open Access version through the publisher's homepage and the Online Catalogue of the State and University Library of Goettingen

(http://www.sub.uni-goettingen.de). Users of the free online version are invited to read, download and distribute it. Users may also print a small number for educational or private use. However they may not sell print versions of the online book.

Satz und Layout: T. E. Venkata Balaji

Umschlaggestaltung: Jutta Pabst

Titelabbildung: T. E. Venkata Raghavan

(C) 2010 Universitätsverlag Göttingen

http:/ / univerlag.uni-goettingen.de

ISBN: 978-3-941875-32-6 
to

my father

Shri T. E. Parthasarathy 



\section{Contents}

$\begin{array}{ll}\text { Preface } & \text { ix }\end{array}$

Introduction $\quad$ xi

The Goals of this Book xxi

A Historical Note $\quad$ xxiii

1 Classification of Annuli and Elliptic Curves 1

1.1 Overview of this Chapter . . . . . . . . . . . 1

1.2 Preliminaries . . . . . . . . . . . . . . . . . 2

1.2.1 Topological Coverings . . . . . . . . . . . . . 2

1.2.2 Branched and Unbranched Coverings of Riemann

Surfaces .................... 4

1.2.3 Relations between $\pi_{1}(M)$ and Coverings of $M$. . 6

1.3 Uniformization of Riemann Surfaces . . . . . . . . . . 8

1.3.1 The Fundamental Theorem . . . . . . . . . . . . . 9

1.3.2 Surfaces with Universal Covering the Sphere . . . . 10

1.3.3 Surfaces with Universal Covering $\mathbb{C}$. . . . . . . . . 10

1.3.4 Surfaces with abelian $\pi_{1}$ and Covering $\boldsymbol{U} \ldots \ldots .11$

1.4 Classification of Annuli up to Conformal Equivalence . . . . 15

1.5 Classification of Elliptic Curves . . . . . . . . . . . . 17

1.5.1 Set-theoretic Classification of Elliptic Curves . . . . 17

1.5.2 Quotients, Projective Embeddings and Automorphic Functions . . . . . . . . . . . . . . 19

1.5.3 The Riemann Surface Structure on $U / \operatorname{PSL}(2, \mathbb{Z}) \ldots 30$

2 Families: Global Deformations 33

2.1 Overview of this Chapter . . . . . . . . . . . . . 33

2.2 Differentiable Families . . . . . . . . . . . . . . . . . 34

2.2.1 The Definition of a Differentiable Family . . . . . . 34 
2.2.2 Examples of Differentiable Families . . . . . . . . . 36

2.2.3 Notions of Triviality and Operations on Differentiable Families . . . . . . . . . . . . 37

2.3 The Fundamental Idea of Kodaira-Spencer . . . . . . . . . . 40

2.3.1 The Local Triviality of Differentiable Families . . . . 40

2.3.2 $C^{\infty}$-Deformations of Complex Structure . . . . . . 40

2.4 Complex Analytic Families . . . . . . . . . . . . . 45

2.4.1 The Definition of a Complex Analytic Family . . . . 46

2.4.2 Examples of Complex Analytic Families . . . . . . . 48

2.4.3 Notions of Triviality and Operations on Complex Analytic Families . . . . . . . . . . . . . . . . . . 48

2.4.4 Remarks on Holomorphic Deformations of Complex Structure ................. 50

2.5 Functorial Properties of Families . . . . . . . . . . . 51

2.5.1 The Functor of Families . . . . . . . . . . . . . 51

2.5.2 The Functor of Equivalence Classes of Families . . . 53

2.6 Two Motivating Examples of Families of Complex Tori . . . 53

2.6.1 The Complex Analytic Family $\mathcal{B}$ of Complex Tori . 53

2.6.2 The Complex Analytic Family $\mathcal{C}$ of Complex Tori . . 54

2.7 Algebraizability and Analytic Deformations . . . . . . . . 57

2.7.1 Algebraizability of Complex Tori . . . . . . . . 58

2.7.2 Non-algebraic Deformations of Complex Algebraic Tori .................... 59

2.8 Discontinuous/Continuous Variation of Complex Structure . 60

2.8.1 Continuous Variation of Complex Structure . . . . . 60

2.8.2 Discontinuous Variation of Complex Structure: Jump Phenomena .............. 60

3 Theory of Local Moduli: Infinitesimal Deformations 63

3.1 Overview of this Chapter . . . . . . . . . . . 63

3.2 Infinitesimal Deformations \& Deformation Maps of KodairaSpencer ................. 66

3.2.1 Infinitesimal Deformations for Differentiable Families . . . . . . . . . . . . . . 66

3.2.2 Infinitesimal Kodaira-Spencer Maps for Differentiable Families . . . . . . . . . . . . . . . 68

3.2.3 The Fundamental Sequence of Vector Bundles for a Differentiable Family . . . . . . . . . . . . 69

3.2.4 Reformulation of the Definition of Differentiable Family in Terms of Differentiable Fiber Bundles . . 70

3.2.5 The Fundamental Sequence of Sheaves for a Differentiable Family . . . . . . . . . . . . . 
3.2.6 The Global Kodaira-Spencer Map for a Differentiable Family . . . . . . . . . . . . . . 72

3.2.7 Local Triviality and the Kodaira-Spencer Maps . . . 73

3.2.8 Infinitesimal Deformations and Kodaira-Spencer Maps for Complex Analytic Families . . . . . . . . 76

3.2.9 Relationships of the Infinitesimal Kodaira-Spencer Maps for a Complex Analytic Family to those of the Underlying Differentiable Family . . . . . . . . . . .

3.2.10 The Global Kodaira-Spencer Map for a Complex Analytic Family . . . . . . . . . . . . .

3.2.11 The Relationship of the Global Kodaira-Spencer Map for a Complex Analytic Family to that of the Underlying Differentiable Family . . . . . . . . . . . . 84

3.3 Behaviour of Infinitesimal Kodaira-Spencer Maps Under Change of the Parameter . . . . . . . . . . . . . . 89

3.3.1 "Chain Rule" for Derivative of Complex Structure . 89

3.3.2 Indispensability of the Assumption of Regularity . . 91

3.4 The Theorems of Completeness and Existence . . . . . . . . 92

3.4.1 Primary Obstructions to Infinitesimal Deformations 92

3.4.2 Complete Families \& the Theorem of Completeness 96

3.4.3 Effective Families and the Number of Moduli . . . . 98

3.4.4 Examples of Complete Effectively Parametrized Families: The Case of Complex Tori . . . . . . . . . 101

3.4.5 Obstructions to Infinitesimal Deformation of Complex Structure: A Reformulation . . . . . . 105

3.4.6 The Theorem of Existence and Number of Moduli . 110

3.5 Deformations of Complex Fiber Bundles . . . . . . . . . . . 112

3.5.1 Differentiable and Complex Analytic Families of Complex Fiber Bundles . . . . . . . . . . . . . . 112

3.5.2 Fundamental Sequences and Diagrams for Families of Complex Fiber Bundles . . . . . . . . . . . . . . . 114

3.5.3 The Global and Infinitesimal Kodaira-Spencer Maps for a Family of Complex Fiber Bundles . . . . . . . 116

3.6 Kuranishi's Theorem and Local Moduli Spaces . . . . . . . 119

3.6.1 Deformations of Complex Analytic Spaces . . . . . . 119

3.6.2 The Infinitesimal Kodaira-Spencer Maps for an Analytic Family of Complex Analytic Spaces . . . . 121

3.6.3 Kuranishi's Theorem and Local Moduli Spaces . . . 121

3.7 Examples of Local Moduli Spaces . . . . . . . . . . . . . 123

3.7.1 Riemann's Formula: Local Moduli for Curves . . . . 123

3.7.2 An Example of a Singular Local Moduli Space . . . 124

3.7.3 Local Moduli Spaces for Complex Tori . . . . . . . . 124 
3.8 Deformations and Local Moduli for Vector Bundles over a

Compact Riemann Surface . . . . . . . . . . . . . . . 125

3.8.1 Families of Vector Bundles and the Infinitesimal

Deformation Maps . . . . . . . . . . 125

3.8.2 Local Moduli for Simple Vector Bundles over a

Compact Riemann Surface . . . . . . . . . . . 126

3.9 Deformation of Schemes and Geometric Vector Bundles over

Schemes . . . . . . . . . . . . . 127

3.9.1 Algebraic Families of Schemes and Vector Bundles . 128

3.9.2 Infinitesimal Deformation Maps for Algebraic

Families of Schemes . . . . . . . . . . . 130

3.9.3 Infinitesimal Deformation Maps for Algebraic

Families of Algebraic Vector Bundles . . . . . . . . . 131

4 Theory of Global Moduli: Fine Moduli Spaces and Coarse Moduli Spaces

133

4.1 Overview of this Chapter . . . . . . . . . . . . 133

4.2 The General Definition of a Family . . . . . . . . . . . . 136

4.2.1 Classification Problems and Families . . . . . . . . 136

4.2.2 Conditions on the Parameter Category $\mathcal{C}$. . . . . 136

4.2.3 Definition of a Functor of Families . . . . . . . . . 137

4.2.4 Remarks on the Above Definition . . . . . . . . . 137

4.2.5 The Functor of Equivalence Classes of Families . . . 138

4.3 Examples of Functors of Families . . . . . . . . . . . . . . 139

4.3.1 Example: Problem of Moduli of a Compact Complex

Manifold ................ . . . 139

4.3.2 Example: Problem of Moduli of Vector Bundles on a Compact Riemann Surface . . . . . . . . . . . . 139

4.3.3 Example: Problem of Moduli of Elliptic Curves . . . 142

4.4 Representable Functors . . . . . . . . . . . . . . . . 143

4.4.1 Yoneda's Lemma . . . . . . . . . . . . . . . . . 143

4.4.2 Examples of Representable Functors . . . . . . . . . 145

4.5 Functorial Definitions: Moduli Problems, Fine Moduli Spaces and Coarse Moduli Spaces . . . . . . . . . . . . . 148

4.5.1 Moduli Problems . . . . . . . . . . . . . . . 148

4.5.2 Examples of Moduli Problems . . . . . . . . . . . 149

4.5.3 Fine Moduli Spaces . . . . . . . . . . . . . . . 150

4.5.4 Coarse Moduli Spaces . . . . . . . . . . . . . . . 152

4.5.5 When Coarse Moduli Spaces are Fine Moduli Spaces 156

4.6 Example of a Fine Moduli Space: Holomorphic Line Bundles and the Jacobian . . . . . . . . . . . . . . 156

4.6.1 The Picard Group of a Ringed Space . . . . . . . . . 156 
4.6.2 The Chern Class of a Line Bundle . . . . . . . . . 157

4.6.3 The Degree of a Vector Bundle . . . . . . . . . . 159

4.6.4 The Moduli Problem for Degree Zero Line Bundles . 161

4.6.5 Construction of the Jacobian . . . . . . . . . . . 162

4.6.6 Local Moduli for Degree Zero Line bundles and the Poincaré Family . . . . . . . . . . . . . . . . . 163

4.6.7 Fine Moduli for Line Bundles . . . . . . . . . . . . . 165

4.7 The Necessity of the Concept of a Coarse Moduli Space: The Example of Elliptic Curves . . . . . . . . . . . . . . . 168

4.7.1 Local Moduli for Elliptic Curves . . . . . . . . . . 169

4.7.2 The Elliptic Modular Function $J_{F}$ Associated to a Family $F \ldots \ldots \ldots \ldots$

4.7.3 The Coarse Moduli Space for Elliptic Curves _ . . . 172

4.8 The Non-existence of Fine Moduli for Elliptic Curves . . . . 177

4.8.1 Local Obstructions to Existence of a Tautological Family . . . . . . . . . . . . . . . . 177

4.8.2 Global Obstructions to Existence of a Fine Moduli Space . . . . . . . . . . . . . . 181

Appendix: Analytic Spaces, Schemes and Cohomology 183

A.1 Sheaves . . . . . . . . . . . . . . . . . . 183

A.2 Locally Ringed Spaces . . . . . . . . . . . . . . 186

A.3 Pullbacks and Pushforwards of Sheaves of Modules . . . . . 186

A.4 Examples of Locally Ringed Spaces . . . . . . . . . . . 187

A.4.1 The Local Model for Differentiable Manifolds . . . . 188

A.4.2 The Local Model for Complex Manifolds . . . . . . . 188

A.4.3 The Local Model for Schemes . . . . . . . . . . . . 188

A.4.4 The Local Model for Complex Analytic Spaces . . . 188

A.5 Manifolds, Analytic Spaces and Schemes as Locally Ringed

Spaces . . . . . . . . . . . . . . . . . . . . . . . 189

A.5.1 Definition of Differentiable Manifold of Class $C^{\infty}$. . 189

A.5.2 Definition of Complex Manifold . . . . . . . . . . 189

A.5.3 Definition of Scheme . . . . . . . . . . . . . 189

A.5.4 Definition of Complex Analytic Space . . . . . . 189

A.6 Some Definitions from Scheme Theory . . . . . . . . . . 190

A.6.1 Affine Schemes and Commutative Rings . . . . . . 190

A.6.2 Integral Schemes . . . . . . . . . . . . . . 190

A.6.3 Schemes of Finite Type over $\mathbb{C}$, Open and Closed

Subschemes . . . . . . . . . . . . . . . . . 191

A.6.4 Local Structure of Closed Subschemes . . . . . . . . 191

A.6.5 Fiber Products . . . . . . . . . . . . . . . . . . 191

A.6.6 Separated Schemes . . . . . . . . . . . . . . 192 
A.6.7 Proper Morphisms and Projective Schemes . . . . . 192

A.6.8 Smooth Schemes of Finite Type over $\mathbb{C}$. . . . . . . 193

A.6.9 Quasi-coherent and Coherent Algebraic Sheaves . . 193

A.7 Some Definitions from Complex Analytic Space Theory . . 194

A.7.2 Proper Morphisms . . . . . . . . . . . . . . . 194

A.7.3 Fiber Products . . . . . . . . . . . . . . . . 195

A.7.5 Coherent Analytic Sheaves . . . . . . . . . . . 195

A.8 Sheaf Cohomology . . . . . . . . . . . . . . . 195

A.9 Čech Cohomology . . . . . . . . . . . . . . . . 198

A.10 Algebraizability of Complex Analytic Spaces . . . . . . . . 199

A.10.1 The Complex Analytic Space Associated to a Scheme of Finite Type Over $\mathbb{C}$. . . . . . . . . . . . . . . 199

A.10.2 The Coherent Analytic Sheaf Associated to a Coherent Algebraic Sheaf . . . . . . . . . 200

A.10.3 Cohomologies of a Coherent Algebraic Sheaf and its Associated Coherent Analytic Sheaf . . . . . . . . 201

A.10.4 Properties of the Associated Complex Analytic Space .................... . 201

A.10.5 The GAGA Correspondence. . . . . . . . . . 202 


\section{Preface}

Broadly speaking, Moduli Theory involves the discovery of rich geometric structures on the set of isomorphism (or suitable equivalence) classes of familiar objects from Geometry, such as manifolds, varieties and bundles over them. This book is a humble and basic introduction to the fundamental notions of family and deformation, together with their role in the construction of moduli spaces. The attempt is to capture the genesis of the main definitions and results of the theory from heuristic ideas. This is done by fostering a mathematical intuition that is grounded in some basic (though nontrivial) motivating examples. These examples serve not only to point to the kind of results one can expect, but also to pathologies that commonly arise in the construction of moduli spaces.

Structure of the Book. A more detailed introduction begins in page xi. An objective and detailed list of the goals of this book is given in page xxi, followed by a brief history of the initial developments in Moduli Theory from page xxiii. There are 4 chapters followed by an Appendix and References.

Target Audience and Prerequisites. This book is addressed to an audience between the Masters level and the Graduate level. One of its guiding principles is to attempt to show that algebra, topology and analysis work together to reveal beautiful geometry, thus giving an integrated view of Mathematics to students at the senior Masters level. More specifically, this book is aimed at the reader who has had some exposure to algebraic geometry, algebraic topology and complex manifold theory. The material from this book has formed the basis for introductory courses on Moduli for doctoral students at the Chennai Mathematical Institute (CMI) and at the Indian Institute of Technology Madras (IIT-M). The material can be covered in two three-month semesters of teaching meeting 4 hours a week with two extra weekly hours for seminars by students.

Exercises and Supplements. The vigilant reader may take up as exercises the verifications of statements whose proofs are claimed to be 
straightforward or routine. Since the author would like to keep the size of the book to a minimum, there are several results in the book whose proofs are referred to other books or articles. The interested reader could take up the study of such material as supplementary reading. A classroom instructor using this book for a course may assign the study of such material to student-groups for discussion and subsequent presentation in seminar sessions running concurrently with the course.

Suggestions for Further Reading. The reader who wishes to further study Classification Theory, Deformation Theory and Moduli Theory may refer to the more advanced texts listed under the References.

Acknowledgements. The author is grateful to the Golden Jubilee Book Writing Scheme of IIT-Madras for its support in the preparation of this book. He is indebted to Prof. Dr. Ulrich Stuhler, Prof. Dr. Ralf Meyer and the Mathematics Institute at the University of Goettingen, Germany, for their encouragement and for supporting the publication of the book. The author is also much thankful to Prof. Dr. Ulf Rehmann of the Department of Mathematics at the University of Bielefeld, Germany, for his encouragement, for forwarding an anonymous review of the first draft of the book and for suggesting that the book be published by the University Press of Goettingen.

November 2009, Chennai

T. E. Venkata Balaji. 


\section{Introduction}

This exposition is presented in four chapters. Each of these chapters begins with an overview of the main topics and results discussed in that chapter. Nevertheless, the purpose of the following discussion is twofold: to serve as a general introduction and also to present the main ideas of each chapter.

Suppose we are given a set of objects in geometry of a certain type i.e., all the objects of this given set are endowed with a fixed type of mathematical structure. Suppose further that it is possible to put an additional mathematical structure on each of these objects; in order to distinguish this additional structure from the structure already present, we will refer to the latter as the underlying structure. The additional structure may have to satisfy some compatibility conditions relative to the underlying structure.

It often happens that there may exist non-isomorphic additional structures on an object i.e., its underlying structure may "admit more than one additional structure". The set-theoretic classification problem is to describe all possible non-isomorphic additional structures that can be imposed on a given underlying structure. This is usually achieved by constructing a natural set-theoretic bijection from the set $S$ of isomorphism classes of additional structures (on the same underlying structure) to a well-known set.

Once this has been achieved, the classification problem may be called a structural classification problem, if we can discover on the set $S$ and on the well-known set, abstract mathematical structures which get identified via the bijection.

For example, consider the underlying structure to be the differentiable manifold $S^{1} \times S^{1} \subset \mathbb{R}^{3}$, which is the familiar torus. This torus can be given the additional structure of a compact complex manifold which makes it into a compact Riemann surface of genus 1, called an elliptic curve. There exist infinitely many non-isomorphic elliptic curves, so that the problem of set-theoretic classification is to describe the set $S$ of isomorphism classes of elliptic curves. 
A bijective mapping called the elliptic modular function $\widetilde{J}$ can be defined on this set $S$ and the values of this function include every possible complex number. Thus, the set $S$ of isomorphism classes of elliptic curves is bijective to the set $\mathbb{C}$ of complex numbers. Given an elliptic curve $E$, the complex number $\widetilde{J}([E])$ associated to its isomorphism class $[E]$ via the elliptic modular function $\widetilde{J}$, is called its $j$-invariant and this number therefore completely characterizes the isomorphism class of the elliptic curve. Next, all the natural mathematical structure on $\mathbb{C}$, (its topology, real and complex manifold structure, scheme structure etc.,) can be transported to the set $S$ via $\widetilde{J}$, but this seems to be artificial.

What happens actually is that there exists a natural structure of Riemann surface $\widetilde{S}$ on $S$, relative to which $\widetilde{J}: \widetilde{S} \longrightarrow \mathbb{C}$ becomes a biholomorphic mapping! In fact, the elliptic modular function $\widetilde{J}: \widetilde{S} \longrightarrow \mathbb{C}$ descends from an elliptic modular function $J: U \longrightarrow \mathbb{C}$ (associated to a natural family of elliptic curves over $U$, where $U$ is the upper halfplane in $\mathbb{C}$ ) because the quotient Riemann surface $\widetilde{S}$ of $U$ by $\operatorname{PSL}(2, \mathbb{Z})$ is set-theoretically the same as $S$ and $J$ is invariant under this action of PSL $(2, \mathbb{Z})$. Hence, as it turns out, there is nothing artificial in transporting the structure on $\mathbb{C}$ to $S$ via $\widetilde{J}$ ! This is the main theme of chapter 1 .

Now let us return to the general discussion i.e., of the classification of nonisomorphic additional structures on a given underlying structure.

If there is a way to make sense of a "variation of the additional structure", then one may investigate if such a variation is continuous or if it is a discrete variation.

If the variation of the additional structure is continuous, (this is usually achieved using the notion of a "family" — we will elaborate later in this introduction, the role of families in the formulation of a classification problem) then we may call the problem of classification as a problem of moduli of the additional structure. Such a problem of moduli poses the following questions: Can the various additional structures (imposed on the same underlying structure) be parametrized by elements of a topological space? Does the parameter topological space have any natural mathematical structure (like that of a manifold, analytic space, scheme etc.,)? If so, how does this structure relate to the variation of the additional structures? How many minimum "independent" parameters are required to completely achieve the parametrization? It is possible to "arrive at" one additional structure from another in a "continuous manner"? What are the additional structures that occur "sufficiently close" to a fixed additional structure?

The study of the local properties around a point of the parameter space is called a local moduli problem and the study of the global properties of the parameter space is called a global moduli problem. 
To illustrate the ideas of the above discussion, let us look at the following situation naturally arising in Geometry. Consider the set of isomorphism classes of compact connected oriented real differentiable 2-dimensional manifolds (surfaces). Any representative surface of an element of this set may be given a system of local complex coordinates which make it into a compact Riemann surface. Thus, the underlying structure is that of a compact connected oriented real differentiable 2-dimensional manifold (surface), and the additional structure that may be imposed is that of a compact Riemann surface.

So, the problem of classification in this situation is to investigate the various distinct structures of compact Riemann surface that can be imposed on a given compact oriented real surface. Of course, the additional structure of Riemann surface imposed must be compatible with the underlying structure of real manifold i.e., any choice of local complex coordinates, giving the additional structure, should also serve as $C^{\infty}$ coordinates.

By a standard theorem for the classification of compact connected oriented real 2-dimensional surfaces, any such surface is topologically isomorphic to a (hollow) sphere attached with a fixed number of (hollow) cylindrical handles. This fixed number is called the genus of the surface. For example, the sphere has genus 0 , and the torus has genus 1 . Therefore the present problem of classification reduces to the study of the various distinct structures of compact Riemann surface that can be imposed on a surface of fixed genus $g$. Any integer invariant of the underlying structure, such as the genus, must obviously be fixed for further classification.

The next question relevant to the present classification problem is whether we can make sense of a variation of complex structures on the same underlying surface of fixed genus. More generally, we may ask what a variation of complex structures on the same underlying real differentiable compact connected manifold could mean.

Recall that a compact complex manifold of dimension $n$ is obtained by glueing (i.e., identifying) open subsets of a finite number of domains in $n$-dimensional complex space by biholomorphic maps called transition functions. These functions are injective holomorphic maps depending on $n$ independent complex variables and take values in $n$-dimensional complex space. It is intuitively clear that, if we allow the transition functions to vary according to an extra variable called the parameter, then corresponding to different values of this parameter we may hope to obtain different compact complex manifolds (in spite of the fact that we are glueing the same finite set of domains, since the nature of the identifications, which depend on the transition functions, would change according to the parameter). Thus, as the parameter varies, say the parameter is a variable point on a parameter space — a complex or differentiable manifold — we would obtain a 
collection of compact complex manifolds, one for each point of the parameter space. Then this collection can be thought of as a family of compact complex manifolds varying with a parameter. We may call the family a differentiable family (respectively a complex analytic family) if the transition functions depend on the parameter differentiably (respectively holomorphically). But the question now is whether all the compact complex manifolds of such a family have (up to isomorphism) the same underlying differentiable manifold structure - for only then can we think of this family as a family of varying complex structures on the same underlying differentiable manifold.

Hence we reduce to the following question: how do we formulate the notion of a family so as to make the ideas of the above paragraph precise? To begin with, it is natural to think of the various compact complex manifolds of a family as fibers of a differentiable (respectively holomorphic) map from a differentiable (respectively complex) manifold (whose local coordinates induce complex structures on the fibers) into a parameter manifold which is also a differentiable (respectively complex) manifold.

But then, what is the condition on this differentiable (respectively holomorphic) map, which will ensure that the underlying differentiable manifold structures of all the fibers are mutually diffeomorphic i.e., to ensure that the fibers are various complex structures on the same underlying differentiable manifold?

This is where the beautiful idea of K.Kodaira and D.C.Spencer comes in. They realized that the only condition on the map that is necessary is that its Jacobian matrix at each point of the source manifold must be of maximal rank. With this condition, they show that for a family of compact connected complex manifolds, not only are the underlying differentiable manifolds of the fibers of this family mutually diffeomorphic, but also that fibers sufficiently close to a given fiber are all compact complex manifolds obtained by glueing the same finite set of domains in complex $n$-dimensional space but by different transition functions which depend on a variable point on the parameter manifold.

This discovery of Kodaira-Spencer is the starting point for their Theory of Deformations of Complex Structures and is the main theme of chapter 2 .

Suppose we are given a family of complex structures parametrized by a base manifold. Given a point of the base, we know that the complex structure on the fiber over this point depends on the transition functions corresponding to this point. Therefore, it is natural to expect that, given a tangent direction at this point, the derivatives of these transition functions along that direction should "measure the rate of change of complex structure" of the fiber with respect to the parameter at that point. This is how the infinitesimal deformation of the complex structure of a fiber of 
a family along a prescribed tangent direction on the base arises, and when it is expressed in an intrinsic form free of the local coordinates on the base and those on the fiber, gives an element of the first cohomology group of the fiber (as compact connected complex manifold) with values in its sheaf of germs of holomorphic vector fields. The study of this formulation and its properties is the main theme of chapter 3 .

The main theme of chapter 4 is to construct examples of two kinds of global moduli space: fine moduli space and coarse moduli space. The method of construction used to obtain such global moduli spaces may be called a passage from the local moduli spaces to a global moduli space, which involves "glueing of various local moduli spaces". We illustrate some of the main ideas of chapter 4 in the following discussion on the problem of moduli of compact Riemann surfaces of fixed genus.

We first explain the Teichmüller-Bers construction. Let $\mathcal{M}_{g}$ denote the set of isomorphism classes of compact Riemann surfaces of genus $g \geq 2$. Since the underlying differentiable manifolds, of any two compact Riemann surfaces of the same genus, are isomorphic, the problem of classification is to describe the various distinct additional structures of compact Riemann surface that can be imposed on the underlying structure of differentiable manifold of any compact Riemann surface of genus $g$.

As a preliminary step towards converting this classification problem into a moduli problem, we first give the structure of a topological space to the set $\mathcal{M}_{g}$. It is in this step that the notion of a family is crucially used. To explain how, let $(F, T, p)$ be a complex analytic family of compact Riemann surfaces of genus $g$ parametrized by a complex analytic space $T$. Thus $p: F \longrightarrow T$ is a proper morphism of complex analytic spaces whose Jacobian is of maximal rank at each point of $F$, and each fiber $F_{t}:=p^{-1}(t), t \in T$, is a compact Riemann surface of genus $g$. Given such a family, we can define the following classifying map:

$$
\nu_{(F, T, p)}: T \longrightarrow \mathcal{M}_{g}, t \mapsto\left[F_{t}\right],
$$

where $\left[F_{t}\right]$ denotes the isomorphism class of the compact Riemann surface $F_{t}$ of genus $g$. It is now natural to expect that any "good" structure of topological space that we may want to impose on $\mathcal{M}_{g}$ should make $\nu_{(F, T, p)}$ into a continuous map. Using this as a guideline, we define the topology on $\mathcal{M}_{g}$ to be the strongest (i.e., the finest) such that the classifying maps corresponding to every possible family are all continuous. This topology is called the topology defined by families and the corresponding topological space structure on $\mathcal{M}_{g}$ will be denoted by $\mathcal{M}_{g}^{T O P}$. Note the following universal property on $\mathcal{M}_{g}^{T O P}$ : if $\mathcal{M}_{g}^{\prime}$ is any topological space structure on $\mathcal{M}_{g}$ such that the classifying maps corresponding to each family are all 
continuous, then the set-theoretic identity mapping $1_{\mathcal{M}_{g}}$ on $\mathcal{M}_{g}$ includes a continuous map $\mathcal{M}_{g}^{T O P} \longrightarrow \mathcal{M}_{g}^{\prime}$.

Now that we have topologized $\mathcal{M}_{g}$, we may pose the following modulitheoretic questions which may be divided into two major categories: questions pertaining to the problem of global moduli of compact Riemann surfaces of genus $g$, and questions pertaining to the problem of local moduli of a given compact Riemann surface of genus $g$.

The questions pertaining to the global moduli problem are: What is the fundamental group of $\mathcal{M}_{g}^{T O P}$ ? What are its higher homotopy groups? Is it a noetherian topological space, and if so, what is its dimension? What are the homology groups of $\mathcal{M}_{g}^{T O P}$ with co-efficients in $\mathbb{Z}, \mathbb{R}$ or $\mathbb{C}$, and what are the cohomology groups of $\mathcal{M}_{g}^{T O P}$ with values in naturally occurring sheaves of abelian groups on $\mathcal{M}_{g}^{T O P}$ ? Is there a natural compactification of $\mathcal{M}_{g}^{T O P}$, and if so, what is the nature of each of the boundary points? Does $\mathcal{M}_{g}^{T O P}$ admit the structure of an analytic space or scheme? If so, what are the properties of this structure and how do these relate to families? In particular, if there exist singular points, does there exist a canonical explicit desingularization which also occurs as a moduli space for a more general moduli problem?

Given a compact Riemann surface $X$ of genus $g$, let $m \in \mathcal{M}_{g}$ denote its isomorphism class. Then the problem of local moduli of $X$ poses the following questions: What is the structure of a sufficiently small neighborhood of $m$ ? (Note that each point occurring in a sufficiently small neighborhood of $m$ can be regarded as a local deformation of the complex structure of $X$.) If $\mathcal{M}_{g}^{T O P}$ admits an analytic space structure, atleast in some neighborhood of $m$, then is $m$ a singular point? If so, what is the type of singularity at $m$ ? (This is the same as asking for a local model for the singularity at $m$.) Can the tangent space at $m$ be described explicitly? Can the dimension of this tangent space be computed? What is the relationship of the structure in a neighborhood of $m$ to families of deformations of $X$ ?

Having posed the above questions, let us see the kind of answers we are able to get.

Let $C$ be a compact Riemann surface of genus $g$. Then its fundamental group is independent of its complex structure, so we will let $\pi_{1, g}$ denote the fundamental group of any compact Riemann surface of genus $g$.

The Theorem of Uniformization of Riemann surfaces asserts that there exists a subgroup $G_{U}$, of automorphisms of the upper half-plane $U$ in $\mathbb{C}$, isomorphic to $\pi_{1, g}$, which acts without fixed points and properly discontinuously on $U$ so that the set-theoretic quotient $U / G_{U}$ can be given a natural complex structure of a compact Riemann surface of genus $g$ isomorphic to $C$, and such that the canonical map $U \longrightarrow U / G_{U}$ becomes a holomorphic 
covering map. Of course, $U$ may be replaced by a domain $D \subset P_{\mathbb{C}}^{1}$ which is biholomorphic to $U$ via an automorphism of the extended complex plane $P_{\mathbb{C}}^{1}$, and $G_{U}$ may be identified with the corresponding subgroup $G_{D}$ of automorphisms of $D$ isomorphic to $\pi_{1, g}$, so that we again have $D / G_{D} \cong C$.

There exists a bounded domain $\mathbb{T}_{g} \subset \mathbb{C}^{3 g-3}$, called the Teichmüller Space of genus $g$, which was constructed originally by Teichmüller as an abstract metric space and was shown by him to be homeomorphic to $\mathbb{R}^{6 g-6}$. To each $t \in \mathbb{T}_{g}$, there is attached a domain $D_{t} \subset P_{\mathbb{C}}^{1}$, such that the union $\cup_{t \in \mathbb{T}_{g}}\{t\} \times D_{t}$ is an open set $\Omega_{g}$ in the product $\mathbb{T}_{g} \times P_{\mathbb{C}}^{1}$, and since the projection map $p_{1}: \mathbb{T}_{g} \times P_{\mathbb{C}}^{1} \longrightarrow \mathbb{T}_{g}$ is of maximal rank everywhere, so is the induced map $p: \Omega_{g} \longrightarrow \mathbb{T}_{g}$, so that $\left(\Omega_{g}, \mathbb{T}_{g}, p\right)$ is actually a complex analytic family of domains in $P_{\mathbb{C}}^{1}$ parametrized by the Teichmüller space. Further, for each $t \in \mathbb{T}_{g}$, there is a subgroup $G_{t}$ of automorphisms of $D_{t}$ consisting of Möbius transformations (i.e., automorphisms of $P_{\mathbb{C}}^{1}$ ) and which is isomorphic to $\pi_{1, g}$, which also acts properly discontinuously and without fixed points on $D_{t}$ so that the quotient $D_{t} / G_{t}$ is a compact Riemann surface $C_{t}$ of genus $g$ and every compact Riemann surface of genus $g$ occurs as such a quotient for a suitable $t \in \mathbb{T}_{g}$. The crucial point now is that there exists a subgroup $G$ of automorphisms of $\Omega_{g}$, which is isomorphic to $\pi_{1, g}$, and which acts properly discontinuously and without fixed points on $\Omega_{g}$ in such a way that it leaves each fiber $\{t\} \times D_{t}$ of the family $p: \Omega_{g} \longrightarrow \mathbb{T}_{g}$ invariant and further, the action on any such fiber $\{t\} \times D_{t}$ identified with $D_{t}$ is nothing but the action of $G_{t}$ on $D_{t}$. Thus, the quotient $\mathcal{C}_{g}:=\Omega_{g} / G$ exists as a complex manifold, and the map $p: \Omega_{g} \longrightarrow \mathbb{T}_{g}$ induces a holomorphic map $\pi: \mathcal{C}_{g} \longrightarrow \mathbb{T}_{g}$, whose Jacobian is of maximal rank everywhere, and each of whose fibers $\pi^{-1}(t)=p^{-1}(t) / G \cong D_{t} / G_{t}=C_{t}$ is a compact Riemann surface of genus $g$, so that $\left(\mathcal{C}_{g}, \mathbb{T}_{g}, \pi\right)$ is a complex analytic family of compact Riemann surfaces of genus $g$ called the Teichmüller-Bers family. Actually, the quotient map $\Omega_{g} \longrightarrow \mathcal{C}_{g}$ is a holomorphic covering map and is hence locally biholomorphic, so that the map $\pi$ is locally given by a biholomorphic map $\mathcal{C}_{g} \rightarrow \Omega_{g}$ followed by the projection $p$, from which it is obvious that $\pi$ is of maximal rank at each point of $\Omega_{g}$.

It now happens that the Teichmüller space $\mathbb{T}_{g}$ contains "redundant parameters" i.e., there are many points of $\mathbb{T}_{g}$ over which the fibers of $\pi$ are isomorphic. Teichmüller discovered that the quotient group of the group of automorphisms of $\pi_{1, g}$ by its normal subgroup of inner automorphisms of $\pi_{1, g}$ i.e.,

$$
\Gamma_{g}:=\operatorname{Aut}\left(\pi_{1, g}\right) / \operatorname{Int}\left(\pi_{1, g}\right),
$$

called the Teichmüller modular group of genus $g$ or the mapping class group of genus $g$, has a natural action on $\mathbb{T}_{g}$, so that $C_{t} \cong C_{t^{\prime}}$ if and only if $t$ and $t^{\prime}$ belong to the same $\Gamma_{g}$-orbit. Thus, there is a natural set-theoretic bijection of the quotient $\mathbb{T}_{g} / \Gamma_{g}$ with $\mathcal{M}_{g}$. Further, the properties of the 
action of $\Gamma_{g}$ on $\mathbb{T}_{g}$ allow us to give a natural structure of complex analytic space on this quotient, such that the canonical quotient map from $\mathbb{T}_{g}$ into this quotient is a holomorphic map of complex analytic spaces. It is, in fact, a ramified covering of the quotient, with the points of ramification corresponding to points of $\mathbb{T}_{g}$ which have nontrivial stabilizers. For each $t \in \mathbb{T}_{g}$, the stabilizer subgroup $\Gamma_{g}(t)$ is the subgroup of $\Gamma_{g}$ consisting of elements which fix $t$, and this stabilizer subgroup is a finite group isomorphic in a natural way to the finite group of automorphisms of the compact Riemann surface $C_{t}$. Since there exist fixed points for the action of $\Gamma_{g}$ i.e., there exist points of $\mathbb{T}_{g}$ having nontrivial stabilizers, the quotient $\mathbb{T}_{g} / \Gamma_{g}$ fails to be a manifold i.e., it has singular points. However, this quotient is a Hausdorff connected topological space. Hence, we may give the set $\mathcal{M}_{g}$ the structure of a Hausdorff connected complex analytic space i.e., that of $\mathbb{T}_{g} / \Gamma_{g}$ transported via the natural bijection of this quotient with $\mathcal{M}_{g}$. We shall denote this structure on $\mathcal{M}_{g}$ by $\mathcal{M}_{g}^{H O L}$. But what has this structure got to do with $\mathcal{M}_{g}^{T O P}$ ?

Let $(F, T, p)$ be any complex analytic family of compact Riemann surfaces of genus $g$, so that we have the continuous classifying map $\nu_{(F, T, p)}$ : $T \longrightarrow \mathcal{M}_{g}^{T O P}$ as explained earlier. The Teichmüller-Bers family $\left(\mathcal{C}_{g}, \mathbb{T}_{g}, \pi\right)$ possesses the following locally-universal property: for each $t \in T$, there exists a neighborhood $U_{t}$ of $t$ in $T$, and a holomorphic map $\nu_{t}: U_{t} \longrightarrow \mathbb{T}_{g}$ which when followed by the holomorphic projection map $\mathbb{T}_{g} \longrightarrow \mathcal{M}_{g}^{H O L}$ is set-theoretically the same as the map $\nu_{(F, T, p)}: T \longrightarrow \mathcal{M}_{g}$ restricted to $U_{t}$. Hence, we see that the set-theoretic map $\nu_{(F, T, p)}: T \longrightarrow \mathcal{M}_{g}$ can also be considered as a holomorphic map $\nu_{(F, T, p)}: T \stackrel{T}{\longrightarrow} \mathcal{M}_{g}^{H O L}$. But by the universal property of $\mathcal{M}_{g}^{T O P}$ explained earlier, it follows that the identity map on $\mathcal{M}_{g}$ induces a continuous map from $\mathcal{M}_{g}^{T O P}$ to $\mathcal{M}_{g}^{H O L}$.

Further, if $V$ is an open subset of $\mathcal{M}_{g}^{T O P}$, since the classifying map for the Teichmüller-Bers family is continuous, the inverse image $\widetilde{V}$ of $V$ under this map is an open set in $\mathbb{T}_{g}$, and by the very definition of the quotient topology, its image in $\mathcal{M}_{g}^{H O L}$ is also an open subset. But the underlying set of this image is simply $V$ itself, and hence we see that the identity mapping on $\mathcal{M}_{g}$ induces a continuous map from $\mathcal{M}_{g}^{H O L}$ to $\mathcal{M}_{g}^{T O P}$. Combined with the last line of the previous paragraph, we thus see that the underlying topological structure of $\mathcal{M}_{g}^{H O L}$ is homeomorphic to $\mathcal{M}_{g}^{T O P}$. Thus, we may think of $\mathcal{M}_{g}^{H O L}$ as a complex analytic space structure on the underlying topological space $\mathcal{M}_{g}^{T O P}$. This justifies the appropriateness of the topology on $\mathcal{M}_{g}$ defined via families in the first place.

Hence, for each family $(F, T, p)$ of compact Riemann surfaces of genus $g$ parametrized by a complex analytic space $T$, the classifying map can be thought of as a holomorphic map into $\mathcal{M}_{g}^{H O L}$. This is expressed by 
saying that $\mathcal{M}_{g}^{H O L}$ is a coarse moduli space for compact Riemann surfaces of genus $g$.

Unfortunately, the Teichmüller family $\left(\mathcal{C}_{g}, \mathbb{T}_{g}, \pi\right)$ does not descend to give a family on $\mathcal{M}_{g}^{H O L}$. If such a thing had happened, then we would call $\mathcal{M}_{g}^{H O L}$ a fine moduli space (and the descended family a universal family), which means that every morphism of complex analytic spaces $T \longrightarrow \mathcal{M}_{g}^{H O L}$ is the classifying map associated to some family on $T$ and further, the classifying map corresponding to any family depends precisely on the equivalence class of that family. It can be shown that there cannot exist a fine moduli space for compact Riemann surfaces of genus $g$, or equivalently that there does not exist a universal family of compact Riemann surfaces of genus $g$.

The study of the factors — global and local obstructions — responsible for the non-existence of a fine moduli space is also a related problem. In chapter 4 , we discuss the problem of moduli of compact Riemann surfaces of genus 1 (these are called elliptic curves), both from the complex analytic viewpoint and the algebraic viewpoint, and we also describe the nature of the global and local obstructions to the non-existence of a fine moduli space for this problem.

As far as the properties of $\mathcal{M}_{g}^{T O P}$ are concerned, J. Harer has proved that the cohomology group $\mathrm{H}^{2}\left(\mathcal{M}_{g}^{T O P}, \mathbb{Z}\right) \cong \mathbb{Z}$, and the Stability Theorem that

$$
\mathrm{H}^{k}\left(\mathcal{M}_{g}^{T O P}, \mathbb{Z}\right) \cong \mathrm{H}^{k}\left(\mathcal{M}_{g+1}^{T O P}, \mathbb{Z}\right) \text { if } g \geq 3 k+1 .
$$

He has also proved that $\mathcal{M}_{g}^{T O P}$ is homotopy-equivalent to a simplicial complex of dimension $4 g-4$. Thus, we have the Cohomology Vanishing Theorem that

$$
\mathrm{H}^{i}\left(\mathcal{M}_{g}^{T O P}, \mathbb{Z}\right)=0 \text { if } i \geq 4 g-4 .
$$

Since a compact Riemann surface of genus $g$ corresponds to a smooth algebraic curve of genus $g$ by J.-P.Serre's GAGA Theorem, $\mathcal{M}_{g}$ can also be thought of as the set of algebraic isomorphism classes of curves of genus $g$, and we could formulate an algebraic version of the moduli problem of compact Riemann surfaces of genus $g$, by considering only algebraic families. D.Mumford's Geometric Invariant Theory allows us to construct a coarse moduli space $\mathcal{M}_{g}^{A L G}$ for this algebraic moduli problem, whose underlying complex analytic space is simply $\mathcal{M}_{g}^{H O L} \cdot \mathcal{M}_{g}^{A L G}$ is found to be irreducible and separated, and this is to be expected since these properties are the counterparts of connectedness and Hausdorffness of $\mathcal{M}_{g}^{H O L}$. This invariant-theoretic construction also gives a natural compactification $\overline{\mathcal{M}}_{g}^{A L G}$ of $\mathcal{M}_{g}^{A L G}$, and the boundary points $\overline{\mathcal{M}}_{g}^{A L G} \backslash \mathcal{M}_{g}^{A L G}$ are certain curves of genus $g$, called stable curves, which possess certain well-defined singularities i.e., their underlying complex analytic spaces can 
be thought of as "Riemann surfaces with singularities". It turns out that $\overline{\mathcal{M}}_{g}^{A L G}$ is also a coarse moduli space for a more general moduli problem viz. that of stable curves of genus $g$.

C.S.Seshadri's Geometric Invariant Theory Over Arbitrary Base extends moduli constructions such as the one above to the case when the base field $\mathbb{C}$ is replaced by any commutative ring, or more generally, by a locally noetherian scheme.

Finally, let us remark that the problem of moduli of holomorphic line bundles on any fixed compact Riemann surface (of arbitrary genus) always admits a fine moduli space for its solution, and this is proved in chapter 4 . This result admits a generalisation called the Narasimhan-Seshadri Theorem, references to which may be found in the bibliographies of [7] or [31]. 


\section{The Goals of this Book}

The Goals of this Book are:

(1) to explain the notion of a family and construct nontrivial examples of families;

(2) to explain the notion of an infinitesimal deformation of complex structure;

(3) to compute all the infinitesimal deformations of any complex torus (of arbitrary dimension) and of any holomorphic line bundle of degree zero on a compact Riemann surface;

(4) to explain the definitions and the major results of the Theory of Local Moduli of Kodaira-Spencer and apply these results to justify Riemann's formula for the number of moduli of a compact Riemann surface;

(5) to explain Kuranishi's Theorem and to give some of its applications;

(6) to explain the notion of a local moduli space and give some examples;

(7) to explain the notion of a moduli problem, of a coarse moduli space and of a fine moduli space;

(8) to construct the fine moduli space from local moduli spaces for the problem of moduli of holomorphic line bundles of degree zero on a compact Riemann surface;

(9) to construct the coarse moduli space from local moduli spaces for the problem of moduli of elliptic curves over the field of complex numbers and

(10) to present the Theory of Moduli as a topic whose study draws from and enriches diverse areas of Mathematics. 



\section{A Historical Note}

The term moduli is due to Riemann, who heuristically justified his formula for the dimension, of the "space" of isomorphism classes of compact Riemann surfaces of genus $g \geq 2$, as $3 g-3$ :

"hängt ... von $3 g-3$ stetig veränderlichen Grossen ab, welche die moduln dieser Klasse genannt werden sollen."

The above words are from Riemann's famous paper on Abelian Functions [16] in 1857. In modern mathematical terminology, Riemann's result can be stated as follows: the local moduli space of a compact Riemann surface of genus $g \geq 2$ is smooth of complex dimension $3 g-3$. O.Teichmüller, in the 30's, gave the precise formulation of Riemann's results, and established some of these results using his Theory of Extremal Quasi-Conformal Mappings [19].

The remaining statements formulated by Teichmüller were proved by Lipman Bers in the 60's [29]. The solution to the complex analytic version of the problem of moduli of compact Reimann surfaces of fixed genus was thus completed. In today's mathematical language, the Teichmüller-Bers Theorem may be stated as follows: there exists a coarse moduli space for the problem of moduli of compact Riemann surfaces of any fixed genus $g$; further, this moduli space is obtained naturally as the quotient of the Teichmüller space of genus $g$ by the Teichmüller modular group of genus $g$, and there exists a locally-universal family of compact Riemann surfaces of genus $g$ on this Teichmüller space.

Meanwhile, the next important development in the theory of deformations of complex structures was obtained in 1957 by Fröhlicher and Nijenhuis [10]. Their result may be stated as follows: a compact complex manifold, whose first cohomology group with values in its sheaf of germs of holomorphic vector fields vanishes, does not undergo changes in its complex structure under sufficiently small perturbations.

It was this result which inspired K.Kodaira and D.C.Spencer to pioneer the study of the Theory of Deformations of Complex Structures [6]. They 
developed this theory during the period 1958 through 1960. This theory may be called the Theory of Local Moduli, and it focusses on the study of arbitrarily small deformations of the complex structure of a compact complex manifold. Such arbitrarily small deformations are called infinitesimal deformations. These are by definition, certain first cohomology classes, namely certain elements of the first cohomology group of the compact complex manifold with values in its sheaf of germs of holomorphic vector fields. Kodaira-Spencer Theory is able to show that every first cohomology class of the above cohomology group corresponds to an infinitesimal deformation only under the hypothesis that the second cohomology group of the compact complex manifold, with values in its sheaf of germs of holomorphic vector fields, vanishes.

An infinitesimal deformation may be thought of as a derivative of the complex structure of the compact complex manifold, and hence under the hypothesis just mentioned, we may say that every first cohomology class may be integrated to obtain a smooth deformation (whose derivative of complex structure is precisely the cohomology class we have integrated). This theorem of Kodaira-Spencer is called the Theorem of Existence, and it may be considered as a theorem for the integrability of formal infinitesimal deformations.

The next important contribution to the Theory of Local Moduli is due to Kuranishi in 1962. His deep theorem extends the Theorem of Existence of Kodaira-Spencer mentioned above. Kuranishi's Theorem [14] asserts that every first cohomology class of a compact complex manifold (relative to its sheaf of germs of holomorphic vector fields) can be integrated to obtain a deformation, but in general the deformation so obtained need not be a smooth deformation.

Finally, Kuranishi's Theorem was extended by Grauert [15] to the case of deformations of the complex structure on any complex analytic space.

So far, we have been considering the problem of moduli of a compact complex analytic space from the complex analytic viewpoint. However, when the compact complex analytic space is also algebraizable, since we know that its algebraic structure is determined uniquely up to isomorphism, we can formulate the problem of moduli of its algebraic structure also.

A.Grothendieck has reformulated the foundations of Algebraic Geometry by developing his Theory of Schemes from the late 50's and since then, the language and tools developed by him have been indispensable in the formulation and solution of various moduli problems in Algebraic Geometry.

The notion of a formal deformation of an algebraic object, such as a scheme or a fiber bundle over a scheme, is due to A.Grothendieck. 
Next, in 1968, M.Schlessinger [20] proved an important theorem which ensures the existence of a versal deformation for the algebraic structure of an algebraic object, provided certain simple criteria are satisfied.

Some of the main contributors to the development of the Theory of Global Moduli in Algebraic Geometry and their theories are: D.Mumford for his Geometric Invariant Theory, Artin for his Theory of Algebraic Spaces [30], C.S.Seshadri for his Geometric Invariant Theory over Arbitrary Base and P.Griffiths for his Theory of Period Mappings.

For complete references to the works of the above mathematicians, and for an ocean of references pertaining to developments in Moduli Theory, see the bibliography in [31]. 



\section{Classification of Annuli and Elliptic Curves}

\subsection{Overview of this Chapter}

In this chapter we classify conformal equivalence classes (i.e., holomorphic isomorphism classes) of annuli and elliptic curves (compact connected Riemann surfaces of genus 1 ). This classification is achieved by applying the Theory of Uniformization of Riemann surfaces. The classification of annuli is done in section 1.4, and that of elliptic curves is done in section 1.5

Any Riemann surface can be uniformized in the sense that it is the quotient of a simply connected domain in the extended complex plane by a group of Möbius transformations. The quotient is a universal covering with deck transformation group isomorphic to the fundamental group of the given Riemann surface. This uniformization is introduced in section 1.3 alongwith a description of those Möbius transformations that give rise to deck transformations in the universal covering.

The main aim of section 1.3 is to develop the complete structure of universal coverings of annuli and elliptic curves and in the process we describe the universal coverings of those Riemann surfaces whose fundamental groups are abelian. For this we introduce the notions of parabolic, elliptic, hyperbolic and loxodromic Möbius transformations.

We get, as by-product of the discussion in section 1.3, some important results such as the torsion-freeness of the fundamental group of a Riemann surface; the absence of elliptic elements in the covering group of a Riemann surface and that the complex 1-dimensional tori (elliptic curves) are the only Riemann surfaces whose fundamental groups are free abelian groups on two generators.

Before all this, the fundamental notions of topological and holomorphic covering, ramified covering, universal covering, deck transformation group, covering group and Galois covering are reviewed in section 1.2. The 
relation between conjugacy classes of subgroups of the fundamental group of a Riemann surface to the coverings it has is also recalled.

The main aim of subsection 1.5.2 is to develop the necessary theory required to endow the quotient of the upper half-plane by the unimodular group with the structure of an abstract Riemann surface and show that this structure is biholomorphic to the complex plane. Thus it is indicated there how the quotient of a Riemann surface by a group of biholomorphic automorphisms is naturally again a Riemann surface. The group is assumed to act properly discontinuously, though it may have fixed points. The case of complex tori of arbitrary dimension is discussed in detail.

The notions of Kleinian, Fuchsian, discontinuous and discrete groups are introduced in (1.5.2.3) and (1.5.2.4), and some relationships between these notions are explained. The elliptic modular function is recalled in (1.5.2.6) and it is required in the classification of elliptic curves. Its definition as usual is via the zeros of the derivative of the Weierstrass $\wp$-function. The latter also justifies the name "elliptic curve" by identifying it with the underlying compact Riemann surface of a smooth projective algebraic curve of genus 1 embedded in two-dimensional complex projective space.

The set-theoretic classification achieved moots the question of a relationship between the natural geometry on the parameter space and the geometry of the objects being classified. This leads to the study of "families" - a concept explained in the next chapter.

\subsection{Preliminaries}

This section rapidly reviews fundamental results from the Theory of Coverings of Riemann surfaces. For more details, see [1] and [2]. All the topological spaces considered will be assumed to be path connected, locally path connected, locally simply connected and Hausdorff. All these properties are for example satisfied by any locally-Euclidean space (topological manifold).

\subsubsection{Topological Coverings}

\subsubsection{The Definition of a Topological Covering}

Let $M$ be a topological space. A topological covering of $M$ consists of a topological space $\widehat{M}$ and a continuous map $p: \widehat{M} \longrightarrow M$ such that the following condition holds: every point of $M$ has an open neighborhood whose inverse image under $p$ is a disjoint union of open sets each of which $p$ maps homeomorphically onto the given neighborhood in $M$. Note in particular that $p$ is a surjective local homeomorphism. $\widehat{M}$ is called the covering space and $p$ the covering map associated to this topological covering. 


\subsubsection{Properties of Local Homeomorphisms}

Assume that $p: \widehat{M} \longrightarrow M$ is a local homeomorphism. Let $f: N \longrightarrow M$ be a continuous map admitting two liftings $g_{1}$ and $g_{2}$. This means that the $g_{i}$ are continuous maps from $N$ into $\widehat{M}$ such that $p \circ g_{1}=f=p \circ g_{2}$. If $g_{1}$ and $g_{2}$ assume the same value at some point of $N$, then we can show that they are equal.

Hence $p$ being a local homeomorphism ensures uniqueness of lifting.

In particular, for a (continuous) curve or path $f:[0,1] \subset \mathbb{R} \longrightarrow M$, a lifting $g$ with $g(0)$ prescribed is unique if it exists. We can further show that if two such curves are homotopic with the same end points, and if the liftings at each level of the homotopy exist (for a prescribed lift of the initial point), then the liftings are themselves homotopic with the same end points. In particular this means they have the same final points in $\widehat{M}$.

\subsubsection{The Curve Lifting Property}

If we assume that $p: \widehat{M} \longrightarrow M$ is a topological covering, which is more than assuming that $p$ is a local homeomorphism, then we can show that $p$ has the curve lifting property: every curve in $M$ can be uniquely lifted to one in $\widehat{M}$ starting at a chosen initial point. Hence by the last line of the previous paragraph homotopic curves can be lifted to homotopic curves and we thus get the Monodromy Theorem for the covering $p: \widehat{M} \longrightarrow M$. Conversely, we can prove that a local homeomorphism $p: \widehat{M} \longrightarrow M$ with the curve lifting property is a topological covering.

\subsubsection{The Sheet Number of a Covering}

If $p: \widehat{M} \longrightarrow M$ is a topological covering, then the set-theoretic fibers $p^{-1}(m)$ of $p$ for all $m \in M$ have the same cardinality. This (cardinal) number is called the number of sheets of the covering.

\subsubsection{Coverings Determined by Discrete Proper Local Homeomorphisms}

We have seen above that if $p: \widehat{M} \longrightarrow M$ is a local homeomorphism with the curve lifting property, then $p$ is a topological covering. Alternatively, if it is known that $p$ is a local homeomorphism and is a proper, discrete map, then we can conclude that $p$ is a covering map.

Recall that a map of topological spaces is said to be discrete if each of its fibers (inverse images of singleton subsets) is a topologically discrete subspace of its domain. For example, any nonconstant holomorphic map of Riemann surfaces is discrete (and open too). Also recall that a map of 
locally compact Hausdorff spaces is said to be proper if the preimage of every compact set is compact. For example, every continuous map of a compact locally Euclidean Hausdorff space into any locally compact Hausdorff space is proper. It can be verified that the image of a closed set under a proper map is closed.

Now let $p: \widehat{M} \longrightarrow M$ be a proper discrete local homeomorphism. We quickly indicate how it is a covering map. Let $m \in M$. Then $p^{-1}(m)$ is discrete and compact, hence a finite set of points $\left\{\widehat{m}_{1}, \ldots, \widehat{m}_{n}\right\}$. Since $p$ is a local homeomorphism, for each $j=1, \ldots, n$ there exist an open neighborhood $U_{j}$ of $m$ and an open neighborhood $W_{j}$ of $\widehat{m}_{j}$ such that $p$ maps $W_{j}$ homeomorphically onto $U_{j}$. We may assume $W_{j}$ are pairwise disjoint. Now $\cup_{j=1}^{n} W_{j}$ is an open neighborhood of $p^{-1}(m)$, so its complement $\widehat{F}$ in $\widehat{M}$ is closed. Since $p$ is proper, $F:=p(\widehat{F})$ is closed in $M$. Then $m$ is not in $F$ and $V:=(M-F) \cap\left(\cap_{j=1}^{n} U_{j}\right)$ is an open neighborhood of $m$ whose inverse image under $p$ is a disjoint union of open neighborhoods each of which $p$ maps homeomorphically

onto $V$ QED

\subsubsection{Branched and Unbranched Coverings of Riemann Surfaces}

\subsubsection{Complex Structure on the Covering Space}

Let $M$ be a Riemann surface. Let $p: \widehat{M} \longrightarrow M$ be a topological covering of $M$. Since $p$ is a surjective local homeomorphism, we can transport the complex structure on $M$ to $\widehat{M}$ thereby making $\widehat{M}$ into a Riemann surface and $p$ into a holomorphic mapping. Since $p$ is locally an injective holomorphic map, it is also locally biholomorphic.

Suppose $\widehat{M}$ is equipped with another complex structure so that it is again a Riemann surface relative to which $p$ becomes a holomorphic map. Then $p$ is again locally biholomorphic relative to the new complex structure. Thus the identity map on $\widehat{M}$ gives a holomorphic isomorphism between the two complex structures on $\widehat{M}$.

Hence any topological covering space of a Riemann surface acquires naturally a unique Riemann surface structure that makes the covering map holomorphic.

With the above Riemann surface structure on $\widehat{M}$, we call $p: \widehat{M} \longrightarrow M$ a holomorphic covering of the Riemann surface $M$. 


\subsubsection{Simple Examples of Covering Maps of Riemann Surfaces}

The following are some of the basic examples of coverings of Riemann surfaces:

1. $p_{k}: \mathbb{C}^{*} \longrightarrow \mathbb{C}^{*}$ where $\mathbb{C}^{*}:=\mathbb{C}-\{0\}$ and $p_{k}(z):=z^{k},(k \in \mathbb{N})$;

2. the exponential map from $\mathbb{C}$ to $\mathbb{C}^{*}$;

3. $p: \mathbb{C} \longrightarrow \mathbb{C} / L$ where $L=L(\tau)=\{n+m \tau ; n, m \in \mathbb{Z}\}$, with $\tau \in \mathbb{C}$ such that $\operatorname{Im}(\tau)>0$, is a lattice and $p$ is the canonical projection. $\mathbb{C} / L$ is topologically a torus. It has a canonical complex structure inherited from $\mathbb{C}$ via $p$ locally. It is hence a compact Riemann surface of genus 1 and is in fact the underlying compact Riemann surface of an elliptic algebraic curve. For more details, see example (1.5.2.2.1), (1.5.2.6.1) and (1.5.2.6.2).

\subsubsection{Ramified Coverings}

Now let $p: \widehat{M} \longrightarrow M$ be a proper non-constant holomorphic mapping of Riemann surfaces. A point $\widehat{m} \in \widehat{M}$ is called a branch point or ramification point of $p$ if there is no neighborhood of $\widehat{m}$ restricted to which $p$ is injective. Let $A$ be the set of branch points of $p$. Then this is a discrete set whose complement is open. Hence $A$ is both discrete and closed. Since $p$ is proper, discrete and open, the set $B:=p(A)$ is also closed and discrete. $B$ is called the set of critical values of $p$. Now let $\widehat{M}_{1}$ denote the complement in $\widehat{M}$ of the inverse image of $B$ under $p$, and $M_{1}$ the complement of $B$ in $M$. Then the restriction of $p$ to $\widehat{M}_{1}$ is a proper, nonconstant holomorphic map of Riemann surfaces which is unbranched, i.e., it has no branch points.

It is easily seen that this map is a discrete proper local homeomorphism and so it is a covering map by (1.2.1.5). Hence the number of sheets of the covering (see (1.2.1.4)) is defined and is finite. Let this number be $n$. This means that every value in $M_{1}$ is taken by $p$ exactly $n$ times. But what about values in $B$ ? It turns out that if we consider the (local) multiplicities with which $p$ takes a value in $B$, then $p$ also takes each value in $B$, counting multiplicities, exactly $n$ times. Hence such a proper nonconstant holomorphic map $p: \widehat{M} \longrightarrow M$ is called an $n$-sheeted ramified holomorphic covering map.

Let us apply the results above to a nonconstant meromorphic function on a compact Riemann surface. Incidentally, every known proof of the existence of nonconstant meromorphic functions on an arbitrary Riemann surface is nontrivial. A way out for a compact Riemann surface is to embed it into complex $n$-dimensional projective space $P_{\mathbb{C}}^{n}$ as a complex submanifold for a suitable $n$. Then Chow's Theorem (A.10.5.2) shows that it acquires the additional structure of a smooth projective algebraic curve. 
Now any nontrivial projection onto any subspace $P_{\mathbb{C}}^{1}$ gives rise to a nontrivial meromorphic function and every meromorphic function is obtained in this way.

Any given nonconstant meromorphic function $f$ on a compact Riemann surface can be thought of as a nonconstant proper holomorphic map into the Riemann Sphere $\mathbb{C} \cup\{\infty\}=P_{\mathbb{C}}^{1}$. Applying the results of the first two paragraphs above gives the following.

Any meromorphic function on a compact Riemann surface has as many zeros as it has poles, where the zeros and poles are counted with multiplicities.

As another application of the results of the first two paragraphs above, we may consider a polynomial of degree $n$ as a holomorphic proper self-map of the Riemann sphere. Since it takes any finite value exactly $n$ times, we get the following.

A polynomial of degree $n$ in one variable takes the value $\infty$ exactly $n$ times.

\subsubsection{Holomorphic Liftings}

Before ending this subsection, we make some remarks on the holomorphicity of continuous liftings. Let $p: \widehat{M} \longrightarrow M$ be an unbranched holomorphic map of Riemann surfaces. This is equivalent to assuming that $p$ is a holomorphic local homeomorphism. Now let $f: N \longrightarrow M$ be any holomorphic map of Riemann surfaces and let a lifting of $f$ to a continuous map $g: N \longrightarrow \widehat{M}$ exist. Then it is easily verified that $g$ is also holomorphic.

An important consequence of the above is the following. Let $p_{i}: \widehat{M}_{i} \longrightarrow$ $M$ be unbranched holomorphic maps of Riemann surfaces for $i=1,2$. Then any continuous map $f: \widehat{M}_{1} \longrightarrow \widehat{M}_{2}$ which is fiber-preserving, i.e., satisfying $p_{2} \circ f=p_{1}$, is holomorphic. Therefore any such fiber-preserving map $f$ which is a homeomorphism is also biholomorphic.

\subsubsection{Relations between $\pi_{1}(M)$ and Coverings of $M$}

Let us denote by $\pi_{1}(M)$ the (first) fundamental group or first homotopy group of any path connected topological space $M$. Recall that $\pi_{1}(M)$ consists of homotopy equivalence classes of loops starting and ending at a chosen point of $M$ called the base point. Path connectedness ensures that a change of the base point would alter $\pi_{1}(M)$ only up to an isomorphism. Now let $p: \widehat{M} \longrightarrow M$ be a topological covering. Since $\pi_{1}(-)$ is a covariant functor from the category of topological spaces (and continuous maps) to the category of groups (and group homomorphisms), we get an induced group homomorphism $\pi_{1}(p): \pi_{1}(\widehat{M}) \longrightarrow \pi_{1}(M)$. Due to the unique path lifting 
property and the Monodromy theorem (1.2.1.3), $\pi_{1}(p)$ is injective and its image determines a subgroup of $\pi_{1}(M)$. For the same reasons, $\pi_{1}(M, m)$ acts on the fiber $p^{-1}(m)$ : given $\widehat{m} \in p^{-1}(m)$ and $[\gamma] \in \pi_{1}(M, m),[\gamma] \cdot \widehat{m}$ is the terminal point of the unique lift of $\gamma$ starting at $\widehat{m}$. It is easy to check that this action moves any point of the fiber to any other point, thereby giving a natural identification of the fiber with the coset space $\pi_{1}(M) / \operatorname{Image}\left(\pi_{1}(p)\right)$.

\subsubsection{Universal Coverings}

Let $M$ be a connected manifold. Then there exists a connected, simply connected manifold $\widetilde{M}$ and a covering map $\widetilde{p}: \widetilde{M} \longrightarrow M$. Since $\widetilde{M}$ is simply connected, its fundamental group is trivial. Any such covering $\widetilde{p}: \widetilde{M} \longrightarrow M$ is called a universal covering of $M$. This is because it satisfies the following universal property. Let $p: \widehat{M} \longrightarrow M$ be any covering of $M$. Let $\widehat{m} \in \widehat{M}$ and $\widetilde{m} \in \widetilde{M}$ be given such that $\widetilde{m}$ is mapped by $\widetilde{p}$ to the same point of $M$ as is $\widehat{m}$ by $p: \widehat{M} \longrightarrow M$. Then there exists a unique lifting of $\widetilde{p}: \widetilde{M} \longrightarrow M$ to a continuous map $f: \widetilde{M} \longrightarrow \widehat{M}$ which takes $\widetilde{m}$ to $\widehat{m}$. The proof of this result uses the hypotheses that $\widetilde{M}$ is simply connected, pathwise connected and locally pathwise connected. Hence given any two universal coverings $\widetilde{p}_{i}: \widetilde{M}_{i} \longrightarrow M,(i=1,2)$, there exists a unique fiberpreserving homeomorphism $f: \widetilde{M}_{1} \longrightarrow \widetilde{M}_{2}$. In this sense, the universal covering $\widetilde{p}: \widetilde{M} \longrightarrow M$ is uniquely determined up to a unique isomorphism.

In particular, if $M$ is a Riemann surface, so that we get a unique structure of Riemann surface on its universal covering space $\widetilde{M}$ as indicated in (1.2.2.1), then we call $\widetilde{M}$ the holomorphic universal covering space of $M$.

\subsubsection{Deck Transformations, Galois Coverings and the Fundamental Group}

A covering transformation or deck transformation of a topological covering $p: \widehat{M} \longrightarrow M$ is a fiber-preserving homeomorphism of $\widehat{M}$ onto itself. The set $\operatorname{Deck}_{M}(\widehat{M}, p)$ of deck transformations becomes naturally a subgroup of the group $\operatorname{Aut}(\widehat{M})$ of automorphisms of $\widehat{M}$. If $\alpha \in \operatorname{Aut}(\widehat{M})$, the mapping $\beta \mapsto \alpha \beta \alpha^{-1}$ gives an isomorphism of $\operatorname{Deck}_{M}(\widehat{M}, p)$ with $\operatorname{Deck}_{M}\left(\widehat{M}, p \alpha^{-1}\right)$ inside $\operatorname{Aut}(\widehat{M})$. Thus the deck transformation group is determined up to conjugation in $\operatorname{Aut}(\widehat{M})$.

The covering $p: \widehat{M} \longrightarrow M$ is said to be Galois (or normal or regular) if $\operatorname{Deck}_{M}(\widehat{M}, p)$ acts transitively on every fiber of $p$. This means that the orbit of any point $\widehat{m} \in \widehat{M}$ is the whole fiber $p^{-1}(p(\widehat{m}))$ to which it belongs. For example the universal covering $\widetilde{p}: \widetilde{M} \longrightarrow M$ of a connected manifold 
$M$ is Galois with deck transformation group isomorphic to $\pi_{1}(M)$. In this case the deck transformation group is called the covering group of $M$.

It is clear that this covering group is determined up to conjugation in the full group of automorphisms of the universal covering space.

\subsubsection{Galois Theory of Coverings}

If $p: \widehat{M} \longrightarrow M$ is a covering of the connected manifold $M$ and $\widehat{M}$ is also a connected manifold, then by the very definition of the universal covering $\widetilde{p}: \widetilde{M} \longrightarrow M$, there exists a continuous fiber-preserving map $f: \widetilde{M} \longrightarrow \widehat{M}$. This turns out to be a covering map and hence it is the universal covering for $\widehat{M}$. Thus $G:=\operatorname{Deck}_{\widehat{M}}(\widetilde{M}, f)$ is isomorphic to the fundamental group of $\widehat{M}$ and is a subgroup of $\operatorname{Deck}_{M}(\widetilde{M}, \widetilde{p}) \cong \pi_{1}(M)$.

Suppose we replace $p: \widehat{M} \longrightarrow M$ by an isomorphic covering, i.e., by a covering $\bar{p}: \bar{M} \longrightarrow M$ coming with a homeomorphism $\alpha: \widehat{M} \longrightarrow \bar{M}$ satisfying $p=\bar{p} \circ \alpha$. Then we obtain a conjugate of $G$ in the fundamental group of $M$. Hence an isomorphism class of coverings $[p: \widehat{M} \longrightarrow M]$ uniquely determines a conjugacy class $[G]$ of subgroups of $\pi_{1}(M)$. If $p$ : $\widehat{M} \longrightarrow M$ is Galois, $G$ turns out to be normal in $\pi_{1}(M)$ and hence all of its conjugates coincide.

Conversely, given a subgroup $G$ of $\pi_{1}(M)$, the latter as usual being identified with the covering group of $M$, we let $\widehat{M}:=\widetilde{M} / G$ i.e., the set of orbits of $G$ in $\widetilde{M}$. Endow $\widehat{M}$ with the quotient topology. The covering map $\widetilde{p}: \widetilde{M} \longrightarrow M$ induces a continuous map $p: \widehat{M} \longrightarrow M$ and it is checked that this is a covering of $M$. Then this covering determines a subgroup of the fundamental group of $M$ as above and this subgroup is found to be conjugate to $G$. Replacing $G$ by any of its conjugates in $\pi_{1}(M)$ would lead to a covering isomorphic to $p: \widehat{M} \longrightarrow M$. Further, in the case when $G$ is normal, this covering $p: \widehat{M} \longrightarrow M$ turns out to be Galois and we have $\operatorname{Deck}_{M}(\widehat{M}, p)=\operatorname{Deck}_{M}(\widetilde{M}, \widetilde{p}) / G$. Of course the case when $G$ is the trivial subgroup of the fundamental group of $M$ gives the universal covering $\widetilde{p}: \widetilde{M} \longrightarrow M$ itself!

Thus there is a bijection between the set of conjugacy classes of subgroups of $\pi_{1}(M)$ and the set of isomorphism classes of coverings of $M$.

\subsection{Uniformization of Riemann Surfaces}

In this section we classify all those Riemann surfaces whose fundamental groups are commutative. We recall the following nontrivial result. For a proof, see [1], Chap. IV, Sec. 4. 


\subsubsection{The Fundamental Theorem}

1.3.1.1 Theorem (Classification of Simply-Connected Surfaces). Let $M$ be a simply connected Riemann surface. Then $M$ is conformally equivalent to one and only one of the following:

1. the Riemann sphere $\mathbb{C} \cup\{\infty\}$;

2. the complex plane $\mathbb{C}$;

3. the unit disc $\Delta=\{z \in \mathbb{C} ;|z|<1\}$ or equivalently the upper half-plane $U=\{z \in \mathbb{C} ; \operatorname{Im}(z)>0\}$.

1.3.1.2 Notation. $\quad \mathbf{S L}(2, \mathbb{C})$ denotes the special linear group of $2 \times 2$ complex matrices of determinant 1 . Restricting to real and further to integer entries leads to the subgroups $\mathbf{S L}(2, \mathbb{R})$ and $\mathbf{S L}(2, \mathbb{Z})$ respectively. We define the projective special linear groups $\operatorname{PSL}(2, \mathbb{C}), \operatorname{PSL}(2, \mathbb{R})$ and $\operatorname{PSL}(2, \mathbb{Z})$ as quotients of the respective special linear groups by the normal subgroup $\left\{ \pm I_{2}\right\}$. Each element in $\operatorname{PSL}(2,-)$ can be represented by exactly two matrices of determinant 1 , with one representative being the negative of the other.

We denote by $\mathbf{P} \Delta(2, \mathbb{C})$ the subgroup of $\mathbf{P S L}(2, \mathbb{C})$ represented by upper triangular matrices.

Notice that each Möbius transformation corresponds to one and only one element of PSL $(2, \mathbb{C})$. Thus the groups of biholomorphic automorphisms of the extended plane $\mathbb{C} \cup\{\infty\}$, the upper half-plane $U=\{z \in \mathbb{C} ; \operatorname{Im}(z)>$ $0\}$ and the complex plane $\mathbb{C}$ are respectively identified with $\operatorname{PSL}(2, \mathbb{C})$, $\operatorname{PSL}(2, \mathbb{R})$ and $\mathbf{P} \Delta(2, \mathbb{C})$.

The unimodular group $\mathbf{P S L}(2, \mathbb{Z})$ of Möbius transformations will be the major object of study in (1.5).

We immediately deduce the following from theorem (1.3.1.1).

1.3.1.3 Corollary (Uniformization for Arbitrary Surfaces). Let $M$ be a Riemann surface with universal covering $\widetilde{p}: \widetilde{M} \longrightarrow M$. Then $\widetilde{M}$ can be taken to be exactly one of the simply connected surfaces 1, 2 or 3 of theorem (1.3.1.1). For each of these choices, the corresponding covering group $\operatorname{Deck}_{M}(\widetilde{M}, \widetilde{p})$ - which is isomorphic to $\pi_{1}(M)$-is a group of Möbius transformations that acts properly discontinuously without fixed points and is a subgroup respectively of $\mathbf{P S L}(2, \mathbb{C}), \mathbf{P} \Delta(2, \mathbb{C})$ or $\mathbf{P S L}(2, \mathbb{R})$.

Proof. For the definitions of properly discontinuous actions see (1.5.2.1.1) and (1.5.2.3). It is obvious from the definition of the deck transformation group that it acts without fixed points and properly discontinuously in the cases mentioned QED 
1.3.1.4 Remark. The covering group of a Riemann surface as determined by the above corollary actually turns out to be topologically discrete. This will be proved independently in (1.5.2.4). However, we shall use this result in our arguments below.

\subsubsection{Surfaces with Universal Covering the Sphere}

Consider a Riemann surface $M$ which admits a covering by the Riemann sphere $\mathbb{C} \cup\{\infty\}$. Since the Riemann sphere is simply connected, any such covering is a universal covering. Now any holomorphic automorphism of the Riemann sphere is a Möbius transformation. If it is not trivial, it has at least one and at most two fixed points. Such a map cannot be a deck transformation. Thus the deck transformation group (which is isomorphic $\left.\pi_{1}(M)\right)$ must be trivial. So $M$ must be simply-connected. But then the identity mapping serves as a universal covering map for $M$. The universal property satisfied by a universal covering (1.2.3.1) then leads to the following.

1.3.2.1 Theorem. Any Riemann surface which admits a covering by the Riemann sphere may be identified with the Riemann sphere itself. With this identification, the only possible covering maps are elements of $\operatorname{PSL}(2, \mathbb{C})$.

\subsubsection{Surfaces with Universal Covering $\mathbb{C}$}

Let us look at those Riemann surfaces $M$ whose universal covering space is $\mathbb{C}$. Thus the deck transformation group (which is isomorphic to $\pi_{1}(M)$ ) must be a subgroup of $\mathbf{P} \Delta(2, \mathbb{C})$ which acts properly discontinuously without fixed points. Now the only fixed-point-free automorphisms of $\mathbb{C}$ are of the form $z \mapsto z+a, a \in \mathbb{C}^{*}:=\mathbb{C}-\{0\}$. Further if $z \mapsto z+a$ is a deck transformation, then so is $z \mapsto z+n a$ where $n \in \mathbb{Z}$. This shows that the deck transformation group is a discrete $\mathbb{Z}$-module. If we identify the automorphism $z \mapsto z+a$ with $a \in \mathbb{C}$, then the deck transformation group becomes a discrete $\mathbb{Z}$-submodule of $\mathbb{C}$. The following characterization of such discrete submodules is easy to prove (see for e.g., [4], Chap.7, Sec.2).

1.3.3.1 Proposition. A discrete $\mathbb{Z}$-submodule of $\mathbb{C}$ consists of either zero alone, or of the integral multiples nw of a single nonzero complex number $\omega$, or of all linear combinations $n \omega+m \tau$ with integral coefficients of two nonzero complex numbers $\omega, \tau$ which have nonreal ratio.

Hence $\pi_{1}(M)$ has to be isomorphic to $(0), \mathbb{Z}$ or $\mathbb{Z} \oplus \mathbb{Z}$.

If $\pi_{1}(M)$ is trivial, then $M$ is biholomorphic to $\mathbb{C}$ itself. 
If $\pi_{1}(M)$ is isomorphic to $\mathbb{Z}$, let $z \mapsto z+a$ be a generator of this infinite cyclic group. Since the deck transformation group is determined up to conjugation inside the group of automorphisms of $\mathbb{C}$ by (1.2.3.2) and since $z \mapsto z+a$ is conjugate to $z \mapsto z+1$ in that group, we may assume the generator to be $z \mapsto z+1$. In this case it is easy to check that $M$ may be identified with $\mathbb{C}^{*}$ and the universal covering map with $p: \mathbb{C} \longrightarrow \mathbb{C}^{*}$ given by $p(z)=e^{2 \pi i z}$.

Finally, let $\pi_{1}(M)$ be isomorphic to $\mathbb{Z} \oplus \mathbb{Z}$. Then we may take $z \mapsto$ $z+1$ and $z \mapsto z+\tau$ with $\operatorname{Im}(\tau)>0$ as generators of this free abelian group. Thus $M$ is biholomorphic to $\mathbb{C} / L$ where $L$ is the lattice $L=L(\tau)=$ $\{n+m \tau ; n, m \in \mathbb{Z}\}$ in $\mathbb{C}$. Hence $M$ is topologically a torus with complex structure naturally inherited from the usual complex structure on $\mathbb{C}$ via the covering map $\mathbb{C} \longrightarrow \mathbb{C} / L$; for more details see (1.5.2.2.1).

We have thus obtained the following.

1.3.3.2 Theorem. If the holomorphic universal covering space of the Riemann surface $M$ is $\mathbb{C}$, then $M$ is conformally equivalent to $\mathbb{C}, \mathbb{C}^{*}$ or a complex torus. These correspond to the cases for which $\pi_{1}(M)$ is isomorphic to (0), $\mathbb{Z}$ or $\mathbb{Z} \oplus \mathbb{Z}$ respectively.

\subsubsection{Surfaces with abelian $\pi_{1}$ and Covering $U$}

Before we proceed further, we collect some simple technical results concerning Möbius transformations that we will need in the sequel. Let $A$ be a nontrivial Möbius transformation. Then it has one or two fixed points in $\mathbb{C} \cup\{\infty\}$. If we write $A$ explicitly as $A(z)=(a z+b) /(c z+d)$ with $a d-b c=1$ then although $(a+d)$ is not well-defined, its square $(a+d)^{2}$ is. We define

$$
\operatorname{Trace}^{2}(A)=(a+d)^{2} .
$$

In other words, although the matrix trace is not a function on $\mathbf{P S L}(2, \mathbb{C})$, its square is; so thinking of $A$ as an element of $\mathbf{P S L}(2, \mathbb{C})$, it is natural to define $\operatorname{Trace}^{2}(A)$ to be the square of the matrix trace. Now to find the fixed points of $A$, we need to solve $A(z)=z$. This in general results in a quadratic equation with discriminant $d_{A}=\operatorname{Trace}^{2}(A)-4$. We now define $A$ to be

1. parabolic if $d_{A}=0$;

2. elliptic if $d_{A} \in \mathbb{R}$ and $-4 \leq d_{A}<0$;

3. loxodromic if $d_{A} \in \mathbb{C}-\{z \in \mathbb{R} ;-4 \leq z \leq 0\}$;

4. hyperbolic if $d_{A} \in \mathbb{R}$ and $d_{A}>0$. 
Thus a hyperbolic element is loxodromic. Since the trace of a matrix is invariant under conjugation by an invertible matrix, we see that the property of a Möbius transformation being parabolic (respectively elliptic etc.,) is invariant under conjugation by a Möbius transformation. The following lemma clarifies the relationship between such properties and the nature of the fixed points. Its proof is a matter of straightforward computation.

\subsubsection{Lemma. A Möbius transformation}

1. is parabolic if and only if it has one fixed point;

2. is parabolic if and only if it is conjugate to a translation $z \mapsto z+a$;

3. has two fixed points if and only if it is conjugate to $z \mapsto \lambda z$ with $\lambda \neq 0,1$ and further, in this case,

(a) it is loxodromic if and only if $|\lambda| \neq 1$;

(b) it is hyperbolic if and only if $\lambda \in \mathbb{R}, \lambda>0$;

(c) it is elliptic if and only if $|\lambda|=1$;

(d) it is elliptic of finite order if and only if $\lambda$ is a root of unity.

We intend next to look at Riemann surfaces with universal covering the upper half-plane $U=\{z \in \mathbb{C} ; \operatorname{Im}(z)>0\}$. So we need to study Möbius transformations in $\operatorname{Aut}(U)=\mathbf{P S L}(2, \mathbb{R})$. The following results about such Möbius transformations are easy to prove.

1.3.4.2 Lemma. (1) Every loxodromic automorphism of $U$ is hyperbolic. (2) An automorphism of $U$ is elliptic if and only if it fixes a point of $U$. Further, every automorphism of $U$ that is of finite order is elliptic.

Statement (1) follows from the definitions. The proof of the first statement of (2) boils down to using the fact that a quadratic expression in one variable with real coefficients has a nonreal zero if and only if it has negative discriminant. For the second statement of (2), use the previous lemma.

Using the lemmas above and the preceding theorems we deduce the following results.

1.3.4.3 Corollary. The fundamental group of a Riemann surface has no torsion.

1.3.4.4 Corollary. The covering group of a Riemann surface cannot contain elliptic elements.

1.3.4.5 Corollary. The covering group of a Riemann surface with universal covering $U$ contains only parabolic and hyperbolic elements. 
We wish to describe Riemann surfaces with abelian fundamental group and universal covering $U$. To do so we will need the following result.

1.3.4.6 Lemma. Any Riemann surface with nonzero abelian $\pi_{1}$ and universal covering $U$ has cyclic covering group.

Proof. The previous corollary says that the covering group $G$ contains only parabolic and hyperbolic elements. We will show that the following cases are mutually exclusive:

Case 1. $G$ contains an element $A$ that is parabolic.

Case 2. $G$ contains an element $A$ that is hyperbolic.

We first claim that if $A$ and $B$ are any pair of commuting nontrivial Möbius transformations and $A$ is parabolic, then so is $B$. Without loss of generality, we may replace $A$ and $B$ respectively with their conjugates by any fixed Möbius transformation. In doing so, we may further assume that $A(z)=z+1$ by lemma (1.3.4.1). Write $B(z)=(a z+b) /(c z+d)$ with $a d-b c=1$. Then the hypothesis that $A$ and $B$ commute forces $c=0, a d=$ 1 and $d=a$. Thus $B(z)=z+(b / a)$ is parabolic by lemma (1.3.4.1). Hence the claim.

Since $G$ is abelian, the claim above shows that $G$ consists entirely of parabolic elements in Case 1 . Hence Case 1 and Case 2 are mutually exclusive.

Consider Case 1. Conjugate $G$ by an automorphism $C$ of $U$ so that $A$ transforms to $z \mapsto z+1$. Then every other element of $G$ transforms to one of the form $z \mapsto z+\beta$ with $\beta \in \mathbb{R}$. Since $G$ is a $\mathbb{Z}$-module and also a discrete subset of the topological group of automorphisms of $U$, the same properties are true of its conjugate $C \circ G \circ C^{-1}$. By identifying the translation $z \mapsto z+\alpha$ with $\alpha \in \mathbb{C}$, this conjugate is identified with a discrete $\mathbb{Z}$-submodule of $\mathbb{C}$ contained in $\mathbb{R}$. Now proposition (1.3.3.1) shows this conjugate is cyclic and hence the same is true of $G$. This disposes of Case 1.

Consider Case 2. By the discussion preceding Case 1, $G$ contains only hyperbolic elements. By lemmas (1.3.4.1) and (1.3.4.2), either $A$ has the form

$$
A(z)=\lambda(z), \lambda \in \mathbb{R}, \lambda>0, \lambda \neq 1
$$

or $A$ has two distinct real fixed points. In the latter case pick an automorphism $C$ of $U$ that takes these fixed points to 0 and $\infty$. Then the conjugate $C \circ A \circ C^{-1}$ has fixed points $0, \infty$ and is hence of the form $z \mapsto \lambda z$ above. Replacing $G$ with $C \circ G \circ C^{-1}$ if necessary, we may therefore assume without loss of generality that $A(z)$ has the form $z \mapsto \lambda z$ above. Write any $B \in G$ 
as $B(z)=(a z+b) /(c z+d)$ with $a, b, c, d \in \mathbb{R}$ and $a d-b c=1$. Since $A$ and $B$ commute, we get the identity

$$
\frac{a(\lambda z)+b}{c(\lambda z)+d}=\frac{\lambda(a z+b)}{c z+d}
$$

which is valid for all $z \in \mathbb{C} \cup\{\infty\}$. The conditions $\lambda \neq 1$ and $\lambda>0$ force $b=0=c, a d=1$, so $B(z)=a^{2} z$. Since $B$ is hyperbolic, $a^{2} \neq 1,0$. Thus every element of $G$ can be written in the form $z \mapsto \mu z$ with $\mu \in \mathbb{R}, \mu>$ $0, \mu \neq 1$. Identifying $z \mapsto \mu z$ with $\log \mu$, the group $G$ is identified with a discrete $\mathbb{Z}$-submodule of $\mathbb{C}$ contained in $\mathbb{R}$. Again proposition (1.3.3.1) shows $G$ is cyclic QED

1.3.4.7 Theorem. Any Riemann surface with $\pi_{1}$ isomorphic to $\mathbb{Z} \oplus \mathbb{Z}$ has universal covering $\mathbb{C}$ and is isomorphic to a complex torus.

Proof. The only candidates for a universal covering apart from $\mathbb{C}$ are $U$ and $\mathbb{C} \cup\{\infty\}$. The choice $\mathbb{C} \cup\{\infty\}$ is ruled out by theorem (1.3.2.1). The choice $U$ is ruled out by the previous lemma since $\pi_{1}$ is commutative but not cyclic. So the universal covering is $\mathbb{C}$ and the Riemann surface is isomorphic to a complex torus by theorem (1.3.3.2) QED

1.3.4.8 Theorem. Let $M$ be a Riemann surface with $\pi_{1}$ abelian and with universal covering $U$ (or equivalently the unit disc $\Delta$ ). Then $M$ is biholomorphic to exactly one of the following:

1. the unit disc $\Delta$;

2. the punctured unit disc $\Delta^{*}:=\Delta-\{0\}$;

3. an annulus $\Delta_{r}:=\{z \in \mathbb{C} ; r<|z|<1\}$.

Proof. If $\pi_{1}(M)$ is trivial, then $M$ is biholomorphic to $U$ (or equivalently to $\Delta)$ by $(1.2 .3 .3)$.

So let $\pi_{1}(M)$ be nontrivial. Then $G \cong \pi_{1}(M) \cong \mathbb{Z}$ by lemma (1.3.4.6).

By (1.3.4.5), the generator of $G$ is either hyperbolic or parabolic.

If the generator of $G$ is parabolic, we may take it to be $z \mapsto z+1$ (see Case 1 of proof of lemma (1.3.4.6)). With this choice of generator for $G$, $M=U / G$ is identified with $\Delta^{*}$ and the covering map $U \longrightarrow M$ with the map $U \longrightarrow \Delta^{*}$ given by $z \mapsto e^{2 \pi i z}$.

If the generator of $G$ is hyperbolic, we may take it to be $z \mapsto \lambda z, \lambda \in$ $\mathbb{R}, \lambda>0, \lambda \neq 1$ (see Case 2 of proof of lemma (1.3.4.6)). By further conjugating by the element $z \mapsto(-1 / z)$ if needed, we may assume without loss of generality that $\lambda>1$. With this choice of generator for $G, M=U / G$ 
is identified with $\Delta_{r}$ where $r=e^{\left(-2 \pi^{2} / \log \lambda\right)}$ and the covering map $U \longrightarrow M$ with the map $U \longrightarrow \Delta_{r}$ given by $z \mapsto e^{(2 \pi i \log z / \log \lambda)}$ (here $\log$ denotes the principal branch of the logarithm) QED

1.3.4.9 Remarks. In this section we have classified all Riemann surfaces with abelian fundamental groups. Let $M$ be a compact Riemann surface of genus $g>1$. $M$ is topologically a 2-dimensional hollow sphere with $g$ hollow cylindrical handles attached to it as follows: the $2 g$ ends of these cylinders are attached to the boundaries of $2 g$ distinct open discs cut out from the sphere. Then $\pi_{1}(M)$ is the quotient of the free group generated by $2 g$ generators $a_{1}, \ldots, a_{g}, b_{1}, \ldots, b_{g}$ by the smallest normal subgroup containing the element

$$
a_{1} b_{1} a_{1}^{-1} b_{1}^{-1} \ldots a_{g} b_{g} a_{g}^{-1} b_{g}^{-1} .
$$

Thus $\pi_{1}(M)$ is a nonabelian group. For such a Riemann surface $M$ the universal covering space has to be the upper half-plane $U$ with covering group a subgroup of $A u t(U)=\mathbf{P S L}(2, \mathbb{R})$ isomorphic to $\pi_{1}(M)$.

In the next two sections we classify annuli and elliptic curves up to conformal equivalence.

\subsection{Classification of Annuli up to Conformal Equivalence}

Let $r, R$ be positive real numbers with $r<R$. Define $A(r, R)=\{z \in \mathbb{C} ; r<$ $|z|<R\}$. Then $A(r, R)$ is an annulus and is naturally a Riemann surface. We want to find conditions under which two such annuli are biholomorphic.

Moduli Theory seeks to reveal the geometry behind the classification of all possible extra structures (of a certain type) that can be imposed on a geometric object with a fixed underlying structure.

We illustrate this point of view by giving two formulations of the problem of classification of annuli. Let us fix an annulus $M:=A\left(r_{0}, R_{0}\right)$. Then the underlying topological space of any annulus $A(r, R)$ is homeomorphic to that of $M$. Therefore our classification problem for annuli may also be stated as follows.

Parametrize all possible conformally distinct complex structures on the underlying topological space of $M$.

Since each annulus $A(r, R) \subset \mathbb{C}$ is naturally an open subset of $\mathbb{R}^{2}$, it acquires the structure of a differentiable manifold. Then the underlying differentiable structure of any annulus is isomorphic (diffeomorphic) to that of $M$. 
Therefore we may also say that we seek to parametrize all possible conformally distinct complex structures on the underlying differentiable manifold of $M$.

We may simplify the problem of classification of annuli as follows. Since the biholomorphic mapping $z \mapsto(z / R)$ takes $A(r, R)$ onto $A(r / R, 1)$, it is enough to classify annuli of the type $A(r, 1)=\Delta_{r}:=\{z \in \mathbb{C} ; r<|z|<1\}$.

1.4.1 Theorem (Classification of Annuli). The set of conformal equivalence classes of annuli of the form $\Delta_{r}$ is in a natural bijective correspondence with the set $\{r \in \mathbb{R} ; 0<r<1\}$ of real numbers.

Proof. Suppose we are given a biholomorphic map $\phi: \Delta_{r_{1}} \longrightarrow \Delta_{r_{2}}$. By theorem (1.3.4.8), the holomorphic universal coverings are given by $p_{j}$ : $U \longrightarrow \Delta_{r_{j}}$, with $p_{j}(z)=e^{\left(2 \pi i \log z / \log \lambda_{j}\right)}$ where $\lambda_{j}=e^{\left(-2 \pi^{2} / \log r_{j}\right)}>1$ for $j=1,2$. Further, each covering group $G_{j}$ is the infinite cyclic subgroup of automorphisms of the upper half-plane $U$ generated by the hyperbolic Möbius transformation $A_{j}(z)=\lambda_{j} z$. Now $\phi \circ p_{1}: U \longrightarrow \Delta_{r_{2}}$ is also a universal covering of $\Delta_{r_{2}}$ with covering group $G_{1}$. Therefore $G_{1}$ must be a conjugate of $G_{2}$ inside the group of automorphisms of $U$ by (1.2.3.2). Let $T$ be an automorphism of $U$ such that $G_{2}=T \circ G_{1} \circ T^{-1}$.

Since $A_{1}$ generates $G_{1}, T \circ A_{1} \circ T^{-1}$ generates $G_{2}$ and so must equal $A_{2}$ or its inverse. With notations as in (1.3.4), we have $\operatorname{Trace}^{2}\left(A_{1}\right)=$ $\operatorname{Trace}^{2}\left(A_{2}\right)=\operatorname{Trace}^{2}\left(A_{2}^{-1}\right) . \lambda_{j}>1$ now forces $\lambda_{1}=\lambda_{2}$ whence $r_{1}=r_{2}$. The conformal equivalence class of $\Delta_{r}$ is thus uniquely determined by $r$ or equivalently by the corresponding $\lambda=e^{\left(-2 \pi^{2} / \log r\right)}$.

We get the following natural correspondences:

$$
\left\{\Delta_{r} ; 0<r<1\right\} \longleftrightarrow(0,1) \subset \mathbb{R} \text { defined by } \Delta_{r} \leftrightarrow r
$$

and

$$
\left\{\Delta_{r} ; 0<r<1\right\} \longleftrightarrow(1, \infty) \subset \mathbb{R} \text { defined by } \Delta_{r} \leftrightarrow \lambda=e^{\left(-2 \pi^{2} / \log r\right)} .
$$

\section{QED}

1.4.2 Remarks. For the moment, the above correspondences seem to be only set-theoretic. The set $(0,1) \subset \mathbb{R}$ may be called a parameter set, and since it comes with the natural structure of differentiable submanifold of $\mathbb{R}$, it may be called a parameter manifold. The classification of annuli up to conformal equivalence is thus achieved by one real parameter $r \in(0,1)$.

The collection $\left\{\Delta_{r} ; r \in(0,1)\right\}$ may be thought of as a family of annuli parametrized by $(0,1)$. We will make this precise in the next chapter in (2.2.1.1) and (2.2.2.1). It is natural to ask whether the structure (topological, differentiable etc.,) on the parameter space is in any way related to the 
properties of the family it parametrizes. This question is the core of every moduli problem. For example, what is the relationship between the complex structures on the annuli $\Delta_{r}$ and the natural differentiable structure on $(0,1)$ where the parameter $r$ varies? This will be clarified by the study of families in the next chapter.

\subsection{Classification of Elliptic Curves}

This section classifies elliptic curves (complex 1-dimensional tori).

In the first subsection we establish a natural correspondence between the set of conformal equivalence classes of elliptic curves and the set of orbits $U / \operatorname{PSL}(2, \mathbb{Z})$ of the unimodular group $\mathbf{P S L}(2, \mathbb{Z})$ in the upper halfplane $U$. This correspondence is the first step in the discovery of the deep geometry on $U / \mathbf{P S L}(2, \mathbb{Z})$ whose beautiful properties are highlighted in Classical Function Theory, Algebraic and Analytic Number Theory and the Algebraic and Analytic Theory of Moduli of Elliptic Curves. We will touch upon some of these properties in sections 7 and 8 of chapter 4 . The richness of the theory arises from the deep fact that any compact Riemann surface has the structure of a smooth projective algebraic curve, a particular case being that 1-dimensional complex tori, namely compact Riemann surfaces of genus 1 , correspond to elliptic algebraic curves over $\mathbb{C}$ - see (1.5.2.6.2).

In the second subsection we quickly review the theory needed to endow $U / \operatorname{PSL}(2, \mathbb{Z})$ with a natural complex structure. This leads to a parametrization of isomorphism classes of complex 1-dimensional tori by the complex plane $\mathbb{C}$ in the last subsection.

\subsubsection{Set-theoretic Classification of Elliptic Curves}

As noted in the beginning of section 1.4, Moduli theory seeks to reveal the geometry behind the classification of all possible extra structures (of a certain type) that can be imposed on a geometric object with a fixed underlying structure. We again illustrate this point of view by giving two formulations of the problem of classification of elliptic curves.

A topological torus is any topological space that is homeomorphic to a (hollow) sphere with one (hollow cylindrical) handle attached to it. It is thus homeomorphic to the product of two circles: $S^{1} \times S^{1}$. It is connected and compact, with fundamental group isomorphic to $\mathbb{Z} \oplus \mathbb{Z}$.

A real 2-dimensional torus is any differentiable manifold that is diffeomorphic to the natural differentiable submanifold structure on the surface $S^{1} \times S^{1}$ inherited from $\mathbb{R}^{3}$. It is orientable and 2-dimensional.

A complex 1-dimensional torus is obtained as follows. Let $L=L(\tau)=$ $\{n+m \tau ; n, m \in \mathbb{Z}\}$ with $\operatorname{Im}(\tau)>0$ be a lattice in $\mathbb{C}$, where by a lattice 
we mean a $\mathbb{Z}$-submodule of maximal rank. Identifying $n+m \tau \in L$ with the fixed-point-free Möbius automorphism of $\mathbb{C}$ given by the map $z \mapsto$ $z+n+m \tau$ makes $L$ a discrete subgroup of automorphisms of $\mathbb{C}$. Consider the topological quotient $\mathbb{C} / L$ of $\mathbb{C}$ by $L$, which is homeomorphic to a torus with fundamental group isomorphic to $L \cong \mathbb{Z} \oplus \mathbb{Z}$. Since the canonical map $\mathbb{C} \longrightarrow \mathbb{C} / L$ is a covering map, $\mathbb{C} / L$ inherits naturally a unique differentiable structure and a unique complex structure from $\mathbb{C}$. Further details are given in (1.5.2.2.1).

The covering $\mathbb{C} \longrightarrow \mathbb{C} / L$ is universal with covering group the abelian subgroup of automorphisms of $\mathbb{C}$ generated by $z \mapsto z+1$ and $z \mapsto z+\tau$, naturally identified with $L . \mathbb{C} / L$ is thus a compact Riemann surface of genus 1. Given $\tau \in U$ we thus get the associated lattice $L(\tau)$ and the complex torus $\mathbb{C} / L(\tau)$.

Conversely by theorem (1.3.4.7), any Riemann surface $M$ with $\pi_{1}(M) \cong$ $\mathbb{Z} \oplus \mathbb{Z}$ has to have $\mathbb{C}$ for its universal covering space and is a complex torus. Theorem (1.3.3.2) shows $M$ is of the form $\mathbb{C} / L(\tau)$. Therefore our classification problem for elliptic curves may be stated as follows.

Parametrize all possible conformally distinct complex structures on the underlying topological space of a fixed complex torus.

Given $\tau_{1}, \tau_{2} \in U$, it is not hard to find a diffeomorphism between the tori they define.

Hence we may also say that we want to parametrize all possible conformally distinct complex structures on the underlying differentiable manifold of a fixed complex torus.

A partial answer to the above question is given by the following.

1.5.1.1 Theorem (Classification of Elliptic Curves). The set of conformal equivalence classes of complex 1-dimensional tori is in a natural correspondence with the set $U / \mathbf{P S L}(2, \mathbb{Z})$ of $\mathbf{P S L}(2, \mathbb{Z})$-orbits in $U$.

Proof. Suppose we are given a biholomorphic map $f: T_{1} \longrightarrow T_{2}$, where $T_{1}$ and $T_{2}$ are the complex tori defined by $\tau_{1}$ and $\tau_{2} \in U$ respectively. Let $p_{i}: \mathbb{C} \longrightarrow T_{i}$ be the universal covering with deck transformation group $G_{i}$ the subgroup of automorphisms of $\mathbb{C}$ generated by $z \mapsto z+1$ and $z \mapsto z+\tau_{i}$ for $i=1,2$. Let $\widetilde{f}: \mathbb{C} \longrightarrow \mathbb{C}$ be a lifting of $f$ to the universal covering spaces by (1.2.1.2), (1.2.1.3) and (1.2.2.4). The map $\widetilde{f}$ is not unique and may be replaced by $A_{2} \circ \tilde{f} \circ A_{1}$ with $A_{j} \in G_{j}$. $\widetilde{f}$ being an automorphism of $\mathbb{C}$ is an element of $\mathbf{P} \Delta(2, \mathbb{C})$ (see (1.3.1.2)) and is hence of the form $\widetilde{f}(z)=a z+b, a, b \in \mathbb{C}, a \neq 0$. Then $\tilde{f} \circ G_{1} \circ \widetilde{f}^{-1}=G_{2}$ by (1.2.3.2). If $\theta$ is the isomorphism of $G_{1}$ onto $G_{2}$ given by $g \mapsto \tilde{f} \circ g \circ \tilde{f}^{-1}$, then $\theta(z \mapsto z+1)=(z \mapsto z+a)$ and $\theta\left(z \mapsto z+\tau_{1}\right)=\left(z \mapsto z+a \tau_{1}\right)$. Identifying the automorphism of $\mathbb{C}$ given by $z \mapsto z+\alpha$ with $\alpha \in \mathbb{C}, G_{1}$ and $G_{2}$ can 
be regarded as $\mathbb{Z}$-modules and $\theta$ as a $\mathbb{Z}$-linear map with $\theta(1)=a$ and $\theta\left(\tau_{1}\right)=a \tau_{1}$. The matrix of $\theta$ is given by

$$
\Theta=\left[\begin{array}{ll}
\alpha & \beta \\
\gamma & \delta
\end{array}\right],
$$

where $\alpha, \beta, \gamma, \delta \in \mathbb{Z}$ satisfy

$$
\begin{gathered}
a=\theta(1)=\alpha \cdot 1+\beta \cdot \tau_{2}, \\
a \tau_{1}=\theta\left(\tau_{1}\right)=\gamma \cdot 1+\delta \cdot \tau_{2} .
\end{gathered}
$$

Since $\theta$ is an isomorphism of $\mathbb{Z}$-modules, $\Theta$ is an invertible matrix with inverse also having entries in $\mathbb{Z}$. The determinant of $\Theta$ must equal \pm 1 , so $\alpha \delta-\beta \gamma= \pm 1$. The formula

$$
\tau_{1}=\frac{\theta\left(\tau_{1}\right)}{\theta(1)}=\frac{\gamma+\delta \tau_{2}}{\alpha+\beta \tau_{2}}
$$

and the fact that $\tau_{i} \in U$ force $\alpha \delta-\beta \gamma=1$. We have shown that if $\tau_{1}, \tau_{2} \in U$ determine conformal tori, then $\tau_{1}$ is the image of $\tau_{2}$ under the unimodular Möbius transformation given by

$$
A(z)=\frac{\gamma+\delta z}{\alpha+\beta z} \in \mathbf{P S L}(2, \mathbb{Z}) .
$$

Conversely, if $\tau_{1}, \tau_{2} \in U$ belong to the same $\operatorname{PSL}(2, \mathbb{Z})$-orbit, then we may reverse the arguments above to obtain a conformal isomorphism $f: T_{1} \longrightarrow$ $T_{2}$ between the tori they define QED

Our next task will be to study $U / \mathbf{P S L}(2, \mathbb{Z})$. We rapidly review the theory required for this in the next subsection.

\subsubsection{Quotients, Projective Embeddings and Automorphic Functions}

We outline how the orbit space for the action of a discontinuous group of automorphisms on a Riemann surface is naturally again a Riemann surface.

\subsubsection{Quotients for Fixed-point-free Actions}

1.5.2.1.1 Properly Discontinuous Actions. Let $W$ be a complex manifold and $G$ a group of biholomorphic automorphisms of $W$.

Let the action of $G$ be fixed-point-free i.e., let every nontrivial element of $G$ leave no point of $W$ fixed. This is equivalent to every stabilizer subgroup 
$G_{\omega}:=\{g \in G ; g(\omega)=\omega\}$ being trivial for each $\omega \in W$, or to the orbit map $\mu_{\omega}: G \longrightarrow W$ given by $g \mapsto g(\omega)$ being injective for every $\omega \in W$. In this case each orbit is a "set-theoretic copy" of $G$.

Let us further assume that $G$ acts properly discontinuously on $W$. This means that for any compact subsets $K_{1}, K_{2}$ of $W$, there exist only finitely many elements $g \in G$ such that the set $g\left(K_{1}\right) \cap K_{2}$ is nonempty. Thus all but finitely many elements of $G$ displace $K_{1}$ completely away from $K_{2}$.

Under these hypotheses, we have the following fundamental result on quotients.

1.5.2.1.2 Theorem. Let $W$ be a complex manifold and $G$ a group of biholomorphic automorphisms acting on $W$ properly discontinuously without fixed points. Then the set $W / G$ of orbits of $G$ in $W$ naturally inherits the structure of a complex manifold from $W$ in such a way that the canonical set-theoretic projection $\phi: W \longrightarrow W / G$ becomes a holomorphic covering map of $W / G$ with deck transformation group $G$. In particular, if $W$ is simply-connected, then $\phi: W \longrightarrow W / G$ is the holomorphic universal covering for $W / G$ and $\pi_{1}(W / G) \cong G$.

Proof. We indicate a brief sketch of the main steps. First of all, we show that each orbit is a discrete subset of $W$.

Next, for each $w \in W$ we find a sufficiently small complex coordinate neighborhood $U(w)$ which is completely displaced by each nontrivial element of $G$. Here we use the hypotheses that $G$ acts properly discontinuously without fixed points.

The quotient topology is given to $W / G$ so that the natural projection $\phi$ : $W \longrightarrow W / G$ becomes continuous. We check that $W / G$ with this topology is Hausdorff using again the hypothesis that $G$ acts properly discontinuously.

We next check that the map $\phi$ restricted to $U(w)$ induces a homeomorphism onto an open subset of $W / G$ which we denote by $\widehat{U}(\widehat{w})$. Then we verify that the inverse image of $\widehat{U}(\widehat{w})$ under $\phi$ splits into the disjoint union of translates of $U(w)$ by various elements of $G$, and further that each of these translates is mapped homeomorphically by $\phi$ onto $\widehat{U}(\widehat{w})$.

In this way $\phi: W \longrightarrow W / G$ becomes a topological covering with deck transformation group $G$. Since $\phi$ is a local homeomorphism, we may transport the structure of complex manifold on $W$ to $W / G$, thus making $\phi$ a holomorphic covering map of $W / G$ (see (1.2.2.1)) QED

1.5.2.1.3 Remark. We note that the statement of the above theorem and its proof can be easily adapted to any of the following categories:

1. Complex manifolds and holomorphic maps (as done above);

2. Differentiable manifolds and differentiable maps; 
3. Topological spaces and continuous maps.

In each situation, the theorem says that the quotient map is a covering in the corresponding category.

1.5.2.1.4 Fundamental Domains. Again let $W$ be a complex manifold and $G$ a group of biholomorphic automorphisms acting properly discontinuously without fixed points on $W$. By a fundamental domain $F$ for $G$, we mean a subset $F$ of $W$ such that:

1. $F$ is closed;

2. $F$ is the closure of its interior;

3. any interior point of $F$ is the only point in its $G$-orbit lying within $F$;

4. the translates of $F$ by elements of $G$ cover $W$.

If such an $F$ exists, the quotient map of the previous theorem $\phi: W \longrightarrow$ $W / G$ maps $F$ onto $W / G$ and is bijective on the interior of $F$. This helps in concluding that $W / G$ has certain properties provided $F$ has them already. For example, if $F$ is compact or connected, then the same is true of $W / G$.

\subsubsection{Quotients of Complex Lie Groups}

Let $W$ be a group with an operation denoted by $\circ$. If $W$ is also a complex manifold such that the set-theoretic map $W \times W \longrightarrow W$ given by $\left(w_{1}, w_{2}\right) \mapsto w_{1} \circ w_{2}^{-1}$ is holomorphic, then $W$ (along with the operation $\circ$ ) is called a complex Lie group. Adapting this definition to the differentiable and topological categories respectively leads to the notions of real Lie group and topological group.

For any abstract group $G$, the map from $G$ into the group of grouptheoretic automorphisms of $G$ given by $g \mapsto$ (left multiplication by $g$ ) is an injective group homomorphism (Cayley's Theorem). Hence the Lie group $W$ can be thought of as a subgroup of the group of biholomorphic automorphisms of the complex manifold $W$.

A subgroup $G$ of the Lie group $W$ is said to be discrete if the underlying subset of $G$ is a discrete subset of the underlying topological space of $W$. Since such a discrete subgroup $G$ acts properly discontinuously without fixed points on $W$, the natural map $\phi: W \longrightarrow W / G$ becomes a holomorphic covering with deck transformation group $G$ as seen in the previous theorem. If $G$ is normal, $W / G$ naturally becomes a complex Lie group and the map $\phi$ a morphism of Lie groups.

1.5.2.2.1 Example (Tori as Quotients). The complex vector space $\mathbb{C}^{n}$ is a complex commutative Lie group with vector addition as group operation. Since it is $2 n$-dimensional as a real vector space, we may fix a basis 
$\omega:=\left\langle w_{1}, \ldots, w_{2 n}\right\rangle$. Then the subset of $\mathbb{C}^{n}$ defined by

$$
G=\left\{\sum_{j=1}^{2 n}\left(m_{j} w_{j}\right) ; m_{j} \in \mathbb{Z}\right\}
$$

is a lattice in $\mathbb{C}^{n}$ (i.e., a $\mathbb{Z}$-submodule of maximal rank) and moreover, a discrete subgroup of the Lie group $\mathbb{C}^{n}$. G has for its fundamental domain the $2 n$-dimensional real closed "parallelopiped" given by

$$
F=\left\{\sum_{j=1}^{2 n}\left(t_{j} w_{j}\right) ; 0 \leq t_{j} \leq 1\right\} .
$$

$F$ is clearly compact and connected. The canonical quotient $\phi: \mathbb{C}^{n} \longrightarrow$ $\mathbb{C}^{n} / G$ is a covering of Lie groups. $T_{\omega}^{n}:=\mathbb{C}^{n} / G$ is an $n$-dimensional compact connected commutative complex Lie group called an $n$-dimensional complex torus and it has universal covering space $\mathbb{C}^{n}$ with deck transformation group (covering group) $G$. Note that $\pi_{1}\left(T_{\omega}^{n}\right) \cong G$. The $n \times 2 n$ complex matrix whose entries are given by the components of the vectors in the $\mathbb{R}$-basis $\omega$, is called the period matrix associated to $T_{\omega}^{n}$.

For the case $n=1$, we may take $w_{1}=1$ and $w_{2}=\tau$ with $\operatorname{Im}(\tau)>0$. For simplicity of notation we write $\tau$ for $\omega=\langle 1, \tau\rangle$. Then $G$ is the lattice $L=$ $L(\tau)=\{n+m \tau ; n, m \in \mathbb{Z}\}$ with fundamental domain $F$ the fundamental parallelogram with vertices $0,1, \tau+1, \tau$. Further $T_{\tau}^{1}=\mathbb{C} / G$ is a complex one-dimensional torus with universal covering $\mathbb{C}$ and deck transformation group $G \cong \pi_{1}\left(T_{\tau}^{1}\right)=\mathbb{Z} \oplus \mathbb{Z}$. The notion of an algebraic group is the analogue of the notion of a Lie group for the algebraic category of schemes. Since $T_{\tau}^{1}$ has the structure of an algebraic elliptic curve (as we shall see in (1.5.2.6.2)), we may hope for the existence of a group law on this algebraic curve that would make it into an algebraic group; this guess turns out to be true.

1.5.2.2.2 Example (The Möbius Group $\operatorname{PSL}(2, \mathbb{C})$ ). The set $M(2, \mathbb{C})$ of $2 \times 2$ matrices over $\mathbb{C}$ is set-theoretically bijective to $\mathbb{C}^{4}$ and thereby inherits the holomorphic structure of $\mathbb{C}^{4}$.

The determinant function $M(2, \mathbb{C}) \longrightarrow \mathbb{C}$ is a holomorphic (polynomial) map which is multiplicative. The Jacobian matrix corresponding to this map is of full rank at each point of the open submanifold $\mathbf{G L}(2, \mathbb{C})$ and the map restricted to this submanifold is itself a group homomorphism onto $\mathbb{C}-\{0\}$.

By the Implicit Function Theorem, the inverse image in $\mathbf{G L}(2, \mathbb{C})$ of the trivial subgroup namely $\mathbf{S L}(2, \mathbb{C})$, becomes a complex Lie group embedded as a closed submanifold of $\mathbb{C}^{4}$. Its quotient by the discrete subgroup $\left\{ \pm I_{2}\right\}$ 
gives $\mathbf{P S L}(2, \mathbb{C})$ a complex Lie group structure. We will study discrete subgroups of $\mathbf{P S L}(2, \mathbb{C})$ in $(1.5 .2 .4)$.

One of the main aims of this subsection is to develop the necessary theory to equip the set $U / \mathbf{P S L}(2, \mathbb{Z})$ of orbits of $\mathbf{P S L}(2, \mathbb{Z})$ in the upper half-plane $U$ with a natural Riemann surface structure so that the natural map $U \longrightarrow U / \mathbf{P S L}(2, \mathbb{Z})$ becomes holomorphic.

Now $\mathbf{P S L}(2, \mathbb{Z})$ does not act without fixed points on $U$ : by (1.3.4.2) there exist elliptic elements in $\mathbf{P S L}(2, \mathbb{Z})$. Hence theorem (1.5.2.1.2) cannot be applied to obtain a Riemann surface structure on the quotient.

We therefore need to modify the above theorem so that it extends to points which are fixed by nontrivial elements of $\mathbf{P S L}(2, \mathbb{Z})$ i.e., to points which have nontrivial stabilizers. It is for this purpose that we next introduce Kleinian and Fuchsian groups, and also quotients by such groups.

\subsubsection{Discontinuous, Kleinian and Fuchsian Groups}

1.5.2.3.1 Definition. Let $G$ be a subgroup of $\mathbf{P S L}(2, \mathbb{C})$. Let $z_{0} \in$ $\mathbb{C} \cup\{\infty\}$. We say that $G$ acts properly discontinuously at $z_{0}$ if the following hold:

1. the stabilizer subgroup of $G$ at $z_{0}$ i.e., the subgroup of those elements of $G$ which leave $z_{0}$ fixed, is finite;

2 . there exists a neighborhood $U$ of $z_{0}$ which is preserved by every element of $G$ which leaves $z_{0}$ fixed, but is completely displaced by all other elements of $G$.

The subset of points of $\mathbb{C} \cup\{\infty\}$ where $G$ acts properly discontinuously is called the region of discontinuity of $G$ and is denoted by $\Omega(G)$. Its complement in $\mathbb{C} \cup\{\infty\}$ is denoted by $\Lambda(G)$ and is called the limit set of $G$.

$G$ is said to be Kleinian if $\Omega(G)$ is nonempty. A Kleinian group $G$ is further said to be Fuchsian if there is a disc or a half-plane that is preserved by (every element of) $G$.

1.5.2.3.2 Proposition. If $G$ is a Kleinian group, then $\Omega(G)$ is an open subset that is preserved by $G$.

Proof. It is easy to verify that $\Omega(G)$ is preserved by $G$. Pick any point $z_{0} \in \Omega(G)$. If this point has trivial stabilizer, then the definition above gives a neighborhood $U$ of $z_{0}$ which is completely displaced by every nontrivial element of $G$. Obviously $U \subset \Omega(G)$ and each point of $U$ has trivial stabilizer.

Thus the subset of points of $\Omega(G)$ with trivial stabilizers is open in $\mathbb{C} \cup\{\infty\}$. 
If the point $z_{0}$ has a nontrivial (finite) stabilizer, then we claim that there exists a sufficiently small neighborhood of $z_{0}$ in which every other point has trivial stabilizer.

In other words, the set of points of $\Omega(G)$ with nontrivial (finite) stabilizers is discrete in $\Omega(G)$.

The above claim is a consequence of the following simple but powerful lemma. It says that if a group $G$ of biholomorphic automorphisms acts on a Riemann surface $M$ such that the stabilizer subgroup at a point of $M$ is finite, then there exists a disc-like neighborhood of that point preserved by $G$ on which the action of the stabilizer looks like the action of a finite cyclic group of rotations of a disc about its center. Granting this lemma, we see that a point of $\Omega(G)$ with finite nontrivial stabilizer has a neighborhood in which every other point has trivial stabilizer. Since the same is also true for points of $\Omega(G)$ with trivial stabilizers, $\Omega(G)$ is indeed an open subset of $\mathbb{C} \cup\{\infty\}$. QED

1.5.2.3.3 Lemma. Let $V$ be a neighborhood of $0 \in \mathbb{C}$ and $H$ be a group of biholomorphic maps $h_{i}: V \longrightarrow V$ that fix $0 \in \mathbb{C}$ for $1 \leq i \leq n$. Then there exists a simply connected neighborhood $D \subset V$ of $0 \in \mathbb{C}$ that is preserved by $H$ and a biholomorphic map $f: \Delta \longrightarrow D$ from the unit disc $\Delta$ fixing $0 \in \mathbb{C}$ such that the group

$$
\widehat{H}:=\left\{\widehat{h}_{j}:=f^{-1} \circ h_{j} \circ f ; \quad j=1, \ldots, n\right\}
$$

is a finite cyclic group of rotations of $\Delta$ about the origin.

Proof. We divide the proof into two steps.

Step 1: We show that there exists a simply connected neighborhood $D$ of the origin which is preserved by $H$.

Fix an element $h \in H$. Consider the image of a closed disc $\bar{D}_{r}:=\{z \in$ $\mathbb{C} ;|z| \leq r\} \subset V$ under $h$. This image is a convex region if and only if the image of the circle $\partial \bar{D}_{r}$ under $h$ is a convex curve. The latter is true if and only if the argument of the tangent to $h\left(\partial \bar{D}_{r}\right)$ viz. $\left((\pi / 2)+\arg z+\arg h^{\prime}(z)\right)$ is an increasing function of $\arg z$ on $\partial \bar{D}_{r}$. The derivative of this function with respect to $\arg z$ is given by the quantity $\operatorname{Re}\left(\frac{z h^{\prime \prime}}{h^{\prime}}\right)+1$ (note that $h^{\prime} \neq 0$ since $h$ is biholomorphic), which is positive for $z$ sufficiently close to the origin. For sufficiently small $\epsilon, h$ therefore maps every closed disc $\bar{D}_{r}, r<\epsilon$, onto a convex region.

Since $H$ is finite, we may choose $\epsilon$ small enough so that the image of the open $\operatorname{disc} D_{\epsilon}:=\{z \in \mathbb{C} ;|z|<\epsilon\} \subset V$ under every element of $H$ is convex. Now we let $D$ to be the intersection of the various images of this open disc under elements of $H$. Then $D$ is open and convex, whence a 
simply connected neighborhood of the origin. Further $D$ is preserved by $H$ due to its very construction. This completes Step 1.

Step 2: We show that $H$ is a finite cyclic group of "rotations of $D$ about the origin".

By the Riemann Mapping Theorem, $D$ is conformally equivalent to the unit disc $\Delta$ by a map $f: \Delta \longrightarrow D$ such that $f(0)=0$. For any $h_{j} \in H$, the mapping $\widehat{h}_{j}:=f^{-1} \circ h_{j} \circ f$ is a conformal automorphism of $\Delta$ which fixes the origin and hence by the Lemma of Schwarz it is a rotation i.e., $\widehat{h}_{j}(z)=e^{i \theta_{j}} z,\left(0 \leq \theta_{j}<2 \pi\right)$. Note that $\widehat{H}:=f^{-1} \circ H \circ f$ is a group since $H$ is. Therefore $\widehat{H}$ and hence $H$ are commutative groups. Choose the smallest positive angle $\theta_{j}$. Then the corresponding $\widehat{h}_{j}$ (resp. $h_{j}$ ) generates $\widehat{H}$ (resp. $H)$ as a cyclic group and $\widehat{h}_{j}(z)=e^{(2 \pi i / n)} z$ QED

\subsection{Corollary. A Kleinian group is finite or countable.}

Proof. Choose an element $z_{0}$ in $\Omega(G)$ which has trivial stabilizer (such $z_{0}$ exist - in fact the subset of points of $\Omega(G)$ which have nontrivial stabilizer is discrete by the above lemma). Then the orbit of $z_{0}$ is discrete, hence finite or countable. But this orbit is just a set-theoretic copy of $G$ QED

We will explain in (1.5.2.5) how to construct a quotient by a Kleinian group so that the quotient naturally acquires the structure of a Riemann surface. We are interested in the quotient of the upper half-plane $U$ by $\mathbf{P S L}(2, \mathbb{Z})$ but all that we can easily deduce about $\mathbf{P S L}(2, \mathbb{Z})$ is that it is discrete. It is not clear whether $\operatorname{PSL}(2, \mathbb{Z})$ is Kleinian. We next study the relationship between Kleinian and discrete subgroups of $\mathbf{P S L}(2, \mathbb{C})$.

\subsubsection{Discrete Subgroups of $\operatorname{PSL}(2, \mathbb{C})$}

Recall that an abstract subgroup $G$ of $\mathbf{P S L}(2, \mathbb{C})$ is said to be discrete if its underlying set is a discrete subset of the underlying topological space of $\operatorname{PSL}(2, \mathbb{C})$.

\subsection{Lemma. Any Kleinian subgroup $G \subset \mathbf{P S L}(2, \mathbb{C})$ is discrete.}

Proof. If $G$ is finite, it is discrete. If not, $G$ is countable by (1.5.2.3.4). If $G$ had an accumulation point $g \in \mathbf{P S L}(2, \mathbb{C})$, then we may find a sequence $\left\{g_{n}\right\}$ of distinct points of $G$ which tends to $g$. We may replace $\left\{g_{n}\right\}$ by $\left\{g_{n+1}^{-1} \circ g_{n}\right\}$ and assume that $g$ is the identity. The sequence $g_{n}(z)$ then tends to $z$ for any $z \in \mathbb{C}$. Choosing $z \in \Omega(G) \cap \mathbb{C}$ contradicts the Kleinian hypothesis on $G$ QED

From the above lemma we deduce the following important result which we have already used in subsections (1.3.2) through (1.3.4) (see (1.3.1.4)). 
1.5.2.4.2 Corollary. The covering group of a Riemann surface is discrete.

1.5.2.4.3 Caution. There exist discrete subgroups of $\mathbf{P S L}(2, \mathbb{C})$ that are not Kleinian.

For example, the Picard group defined by

$$
\left\{A(z)=\frac{a z+b}{c z+d} \mid a d-b c=1 ; a, b, c, d \text { Gaussian Integers }\right\}
$$

is a discrete but not Kleinian subgroup of $\mathbf{S L}(2, \mathbb{C})$ and the same are true of its image in $\mathbf{P S L}(2, \mathbb{C})$.

However, the notions of discreteness and being Kleinian coincide for a group of conformal automorphisms of a disc (or half-plane). In this case the group is therefore also Fuchsian. Further the preserved disc (or half-plane) is entirely contained in the region of discontinuity. More precisely we have the following.

1.5.2.4.4 Theorem. Let $G$ be a subgroup of automorphisms of the unit disc $\Delta$. Then the following are equivalent:

1. $G$ is discrete;

2. $G$ is Kleinian and acts properly discontinuously at each point of $\Delta$;

3. $G$ is Kleinian and acts properly discontinuously at some point of $\Delta$;

4. $G$ is Kleinian.

Proof. $(1) \Rightarrow(2)$ : Suppose that $G$ does not act properly discontinuously at some point $z_{0}$ of $\Delta$. This holds if and only if there exists an infinite sequence of distinct points $\left\{z_{n}\right\}$ in the $G$-orbit of $z_{0}$ tending to $z_{0}$. For each $n$, pick $g_{n} \in G$ such that $g_{n}\left(z_{n}\right)=z_{0}$. Thus $\left\{g_{n}\right\}$ is an infinite sequence of distinct points of $G$.

We will show that $G$ has an accumulation point, contradicting its discreteness. Define for each nonnegative integer $n$

$$
A_{n}(z)=\frac{z-z_{n}}{1-\overline{z_{n}} z}
$$

Then $\left\{A_{n}\right\}$ is a sequence of automorphisms of $\Delta$ tending to $A_{0}$. For each positive integer $n$, the automorphism of $\Delta$ given by

$$
C_{n}=A_{n+1} \circ g_{n+1}^{-1} \circ g_{n} \circ A_{n}^{-1}
$$

leaves the origin fixed, so Schwarz' lemma implies that $C_{n}(z)=\lambda_{n} z,\left|\lambda_{n}\right|=$ 1. Since the unit circle is complete, we may assume without loss of generality that $\left\{\lambda_{n}\right\}$ converges to an element $\lambda_{0}$ of the unit circle. Then for each $z$ in the unit disc, $C_{n}(z)$ tends to $C_{0}(z)=\lambda_{0} z$. 
Now the sequence of points $\left\{h_{n}=g_{n+1}^{-1} \circ g_{n}\right\}$ of $G$ tends to $A_{0}^{-1} \circ C_{0} \circ A_{0}$. Since the $g_{n}$ are distinct, the sequence $\left\{h_{n}\right\}$ contains an infinite subsequence of distinct points. Therefore we are done.

$(2) \Rightarrow(3)$ : Trivial.

$(3) \Rightarrow(4)$ : Trivial.

$(4) \Rightarrow(1)$ : Already established in (1.5.2.4.1) QED

Let $G$ be a Fuchsian group which preserves the disc $D$. Then the exterior of $D$ is also a disc in $\mathbb{C} \cup\{\infty\}$ that is preserved by $G$. So the exterior of $D$ also lies in the region of discontinuity $\Omega(G)$ by the above theorem. Hence we obtain the following.

1.5.2.4.5 Corollary. The limit set $\Lambda(G)$ of points of $\mathbb{C} \cup\{\infty\}$ where the Fuchsian group $G$ does not act properly discontinuously is contained in the boundary $\partial D$ of the disc $D$ that $G$ preserves.

\subsubsection{Quotients of Domains in $\mathbb{C} \cup\{\infty\}$ by Kleinian Groups}

Let $G$ be a Kleinian group and $V$ a $G$-invariant component of $\Omega(G)$. We wish to endow the set of orbits $V / G$ with a holomorphic structure.

By assumption $G$ acts properly discontinuously at each point of $V$. Give $V / G$ the quotient topology so that the natural map $\phi: V \longrightarrow V / G$ becomes continuous. It will follow from the discussion below that $\phi$ is an open map and that the topological space $V / G$ is Hausdorff.

Suppose that $z$ is a point of $V$ with trivial stabilizer. Then there exists an open neighborhood $U_{z}$ of $z$ which is completely displaced by every nontrivial element of $G$. Choose a local complex coordinate neighborhood $\left(W_{z}, \omega\right)$ centered at $z$ i.e., a neighborhood $W_{z}$ of $z$ which is mapped biholomorphically by $\omega$ onto the unit disc in $\mathbb{C}$ with $z$ mapped onto the origin. Without loss of generality we may assume that $W_{z} \subseteq U_{z}$. Then $\phi$ maps $W_{z}$ homeomorphically onto an open set in $V / G$ which thereby inherits the complex structure on $W_{z}$. In this way, the $G$-invariant open set of points of $V$ whose stabilizers are trivial acquires the structure of a covering space over its quotient (by $G$ ) which is a Riemann surface (see the proofs of (1.5.2.3.2) and (1.5.2.1.2)).

Next let us look at a point $z$ in $V$ with nontrivial (finite) stabilizer. Then there exists an open neighborhood $U_{z}$ of $z$ which is preserved by those elements of $G$ which fix $z$ and which is completely displaced by all other elements of $G$. We have seen that the action of the stabilizer on $U_{z}$ (chosen sufficiently small) looks like the action of a finite cyclic group of rotations about the center of a disc (1.5.2.3.3). Now choose a local complex coordinate neighborhood $\left(W_{z}, \omega\right)$ centered at $z$ so that the action of the generator of the stabilizer on its image (the unit disc) is given by $\omega \mapsto$ 
$e^{(2 \pi i / k)} \omega$, where $k$ is the cardinality of the stabilizer. The homeomorphism $\omega \circ \phi^{-1}$ followed by the self-map $\omega \mapsto \omega^{k}$ of the unit disc, can then be deemed to be a local complex coordinate neighborhood centered at $\phi(z)$ on $V / G$. Note that this works even when $k=1$ and hence also takes care of the case of points with trivial stabilizers that we just discussed above.

Thus $V / G$ gets the structure of a Riemann surface such that $\phi: V \longrightarrow$ $V / G$ is holomorphic. $\phi$ will be ramified at all those points of $V$ that have nontrivial stabilizers (1.2.2.3) and outside this subset of points it will be a covering map (1.5.2.1.2).

Finally we wish to make $\Omega(G) / G$ into a Riemann surface. Declare two components of $\Omega(G)$ to be equivalent under $G$ if there exists an element of $G$ which maps one (biholomorphically) onto the other. Let $\left\{\Omega_{i} ; i \in \mathbb{N}\right\}$ be a maximal set of nonequivalent components of $\Omega(G)$. Then there is a natural set-theoretic bijection of the orbit space $\Omega(G) / G$ with the disjoint union of orbit spaces $\Omega_{j} / G_{j}$ where $G_{j}$ is the subgroup of $G$ that preserves $\Omega_{j}$ for each $j$. Then $G_{j}$ acts properly discontinuously on $\Omega_{j}$, so by the previous paragraph each quotient $\Omega_{j} / G_{j}$ has a natural structure of Riemann surface such that the canonical map $\Omega_{j} \longrightarrow \Omega_{j} / G_{j}$ is holomorphic. Hence we have established the following.

1.5.2.5.1 Theorem. If $G$ is Kleinian group, then $\Omega(G) / G$ is a (countable or finite) union of Riemann surfaces such that the canonical set-theoretic projection $\Omega(G) \longrightarrow \Omega(G) / G$ is holomorphic.

\subsubsection{Projective Embeddings and Algebraizability of Complex 1-dimensional Tori}

1.5.2.6.1 The Weierstrass $\wp$-Function Associated to A Torus. Let $\tau \in U=\{z \in \mathbb{C} ; \operatorname{Im}(z)>0\}$ and let $L=L(\tau)=\{n+m \tau ; n, m \in \mathbb{Z}\}$ be the corresponding lattice. As we have already seen, $T(\tau)=\mathbb{C} / L$ is a complex torus. Define the Weierstrass $\wp$-function associated to this torus by the expression

$$
\wp(z)=\frac{1}{z^{2}}+\sum_{\omega \in L-\{0\}}\left(\frac{1}{(z-\omega)^{2}}-\frac{1}{\omega^{2}}\right) .
$$

Then $\wp(z)$ is an $L$-invariant meromorphic function on $\mathbb{C}$ which has poles of order two precisely at each point of $L$. Therefore it can be considered as a meromorphic function on $T(\tau)$ with a single pole of order two at the point given by the image of $L$. It is not hard to verify (see for e.g., [4], Chap.7, Sec.3) that $\wp(z)$ satisfies the differential equation

$$
\left(\wp^{\prime}(z)\right)^{2}-4(\wp(z))^{3}+g_{2} \wp(z)+g_{3}=0,
$$


where the numbers $g_{2}$ and $g_{3}$ are given by

$$
g_{2}=60 \sum_{\omega \in L-\{0\}}\left(\omega^{-4}\right) \text { and } g_{3}=140 \sum_{\omega \in L-\{0\}}\left(\omega^{-6}\right) \text {. }
$$

1.5.2.6.2 Projective Embedding Using the $\wp$-Function. Consider the homogeneous polynomial of degree 3 in three variables given by

$$
P\left(x_{0}, x_{1}, x_{2}\right)=x_{0} x_{1}^{2}-4 x_{2}^{3}+g_{2} x_{0}^{2} x_{2}+g_{3} x_{0}^{3} .
$$

It is simply the homogenization of the polynomial expression obtained by replacing $\wp^{\prime}(z)$ with $x_{1}$ and $\wp(z)$ with $x_{2}$ on the left-hand side of the differential equation for $\wp(z)$ mentioned above.

The solution set of $P=0$ defines an analytic as well as algebraic hypersurface $\mathcal{C}$ of degree 3 in complex projective 2-dimensional space $P_{\mathbb{C}}^{2}$. Hence $\mathcal{C}$ is called a cubic curve in $P_{\mathbb{C}}^{2}$. Since the discriminant $g_{2}^{3}-27 g_{3}^{2} \neq 0$, at least one of the partial derivatives $\frac{\partial P}{\partial x_{i}}$ is nonzero at each point of $P_{\mathbb{C}}^{2}$. This ensures that $\mathcal{C}$ is analytically as well as algebraically smooth. Further, the (closed) set of points of $\mathcal{C}$ naturally inherits the structure of a compact Riemann surface from the complex structure on $P_{\mathbb{C}}^{2}$.

Now consider the holomorphic mapping $\Phi: T(\tau)-\{\widehat{L}\} \longrightarrow P_{\mathbb{C}}^{2}$ given by $z \mapsto\left[1: \wp^{\prime}(z): \wp(z)\right]$, where $\widehat{L} \in T(\tau)$ is the image of $L$. Since $\wp(z)$ (resp. $\wp^{\prime}(z)$ ) has a pole of order two (resp. of order three) at $\widehat{L}$, rewriting $\Phi$ as $\Phi(z)=\left[z^{3}: z^{3} \wp^{\prime}(z): z^{3} \wp(z)\right]$ extends it holomorphically to $\widehat{L}$. The image of $T(\tau)$ under $\Phi$ is contained in $\mathcal{C}$, and it can be verified that $\Phi$ is a bijective holomorphic map onto $\mathcal{C}$. Thus $\Phi$ is a biholomorphic map through which $T(\tau)$ gets the algebraic structure of a smooth projective cubic curve of genus 1 in $P_{\mathbb{C}}^{2}$ called an elliptic algebraic curve. Conversely given an elliptic algebraic curve, its underlying compact Riemann surface of genus 1 is a complex 1-dimensional torus determined uniquely up to isomorphism by the GAGA correspondence (A.10.5.1).

Hence the classification of complex 1-dimensional tori is the same as the classification of elliptic algebraic curves over $\mathbb{C}$.

1.5.2.6.3 The Automorphic Functions $\lambda$ And $J$. We continue using the notations of (1.5.2.6.2). Our aim is to quickly describe the elliptic modular function. This will be used in the next section where we obtain the structure of a Riemann surface on the set of isomorphism classes of elliptic curves.

Consider the first order differential equation satisfied by the Weierstrass $\wp$-function associated to $\tau \in U$ (1.5.2.6.1). This differential equation may 
be written in the form

$$
\left(\wp^{\prime}(z)\right)^{2}=4\left(\wp(z)-e_{1}\right)\left(\wp(z)-e_{2}\right)\left(\wp(z)-e_{3}\right)
$$

where $e_{1}, e_{2}, e_{3}$ are the zeros of the polynomial $4 Z^{3}-g_{2} Z-g_{3}$. These zeros are distinct and are related to $\tau \in U$ by the formulae

$$
e_{1}=\wp\left(\frac{1}{2}\right), \quad e_{2}=\wp\left(\frac{\tau}{2}\right), \quad e_{3}=\wp\left(\frac{1+\tau}{2}\right) .
$$

We next define the function $\lambda: U \longrightarrow \mathbb{C}$ by

$$
\lambda(\tau)=\frac{e_{3}-e_{2}}{e_{1}-e_{2}}
$$

$\lambda(\tau)$ is analytic and never assumes the values 0 and 1 because $e_{3}-e_{2}, e_{1}-e_{2}$ and $e_{3}-e_{1}$ are analytic functions of $\tau \in U$ that never vanish. The function $\lambda$ is called automorphic because it is invariant under the congruence mod 2 subgroup of the unimodular group $\operatorname{PSL}(2, \mathbb{Z})$ given by

$$
\left\{\left[\begin{array}{ll}
a & b \\
c & d
\end{array}\right] \in P S L(2, \mathbb{Z}) ;\left[\begin{array}{ll}
a & b \\
c & d
\end{array}\right] \equiv\left[\begin{array}{ll}
1 & 0 \\
0 & 1
\end{array}\right](\bmod 2)\right\} .
$$

Finally we define the function $J: U \longrightarrow \mathbb{C}$ by

$$
J(\tau)=\frac{4}{27} \frac{\left(1-\lambda(\tau)+(\lambda(\tau))^{2}\right)^{3}}{(\lambda(\tau))^{2}(1-\lambda(\tau))^{2}}=\frac{-4\left(e_{1} e_{2}+e_{2} e_{3}+e_{3} e_{1}\right)^{3}}{\left(\left(e_{1}-e_{2}\right)\left(e_{2}-e_{3}\right)\left(e_{3}-e_{1}\right)\right)^{2}}=\frac{g_{2}^{3}}{g_{2}^{3}-27 g_{3}^{2}}
$$

Then $J(\tau)$ is analytic on $U$ and is automorphic with respect to the full unimodular group $\operatorname{PSL}(2, \mathbb{Z})$. The domain $F:=\left\{z \in \mathbb{C} ; \frac{-1}{2}<\operatorname{Re}(z)<\frac{1}{2}\right.$, $|z|>1\}$ along with a part of its boundary is mapped by $J$ conformally in a one-to-one manner onto $\mathbb{C}$. Further the closure of $F$ is a fundamental domain for the action of $\mathbf{P S L}(2, \mathbb{Z})$ on $U$. The function $J(\tau)$ is called the elliptic modular function.

\subsubsection{The Riemann Surface Structure on $U / \operatorname{PSL}(2, \mathbb{Z})$}

We put to use the concepts reviewed in the previous subsection to prove the following.

\subsubsection{Theorem (Holomorphic Parametrization of Elliptic}

Curves). The set $U / \mathbf{P S L}(2, \mathbb{Z})$ of isomorphism classes of complex 1-dimensional tori has a natural structure of Riemann surface biholomorphic to $\mathbb{C}$. 
Proof. We have seen in (1.5.1.1) that the set of conformal equivalence classes of complex 1-dimensional tori is bijective in a natural way to $U / \operatorname{PSL}(2, \mathbb{Z})$. Now $\mathbf{P S L}(2, \mathbb{Z})$ is discrete, hence Kleinian and Fuchsian by theorem (1.5.2.4.4). By the same theorem, it acts properly discontinously on $U$. Hence $U / \operatorname{PSL}(2, \mathbb{Z})$ is a Riemann surface such that the canonical $\operatorname{map} U \longrightarrow U / \mathbf{P S L}(2, \mathbb{Z})$ is holomorphic by (1.5.2.5.1). The elliptic modular function $J(\tau)$ on $U$ is invariant under $\mathbf{P S L}(2, \mathbb{Z})$ and hence defines a holomorphic function $\widetilde{J}$ on the Riemann surface $U / \operatorname{PSL}(2, \mathbb{Z})$. The fundamental regions of $\mathbf{P S L}(2, \mathbb{Z})$ and $J(\tau)$ in $U$ are the same (1.5.2.6.3) and therefore $\widetilde{J}: U / \mathbf{P S L}(2, \mathbb{Z}) \longrightarrow \mathbb{C}$ is a biholmorphic mapping QED

1.5.3.2 Remarks. The above study of the problem of classification of elliptic curves illustrates the need for quotient constructions in classification problems. Most often the parameter set obtained in the first attempt towards a parametrization (the upper half-plane $U$ in our study) contains "redundant" parameters (every element of $U$ belonging to a fixed $\operatorname{PSL}(2, \mathbb{Z})$-orbit defines up to isomorphism the same conformal torus). Fortunately there may be a "good" group of automorphisms of the parameter space $(\mathbf{P S L}(2, \mathbb{Z})$ in our study) which permutes the redundant parameters. To get rid of the redundant parameters we must therefore construct a quotient $(U / \mathbf{P S L}(2, \mathbb{Z})$ in our study) by going modulo the group. We would like the quotient to have the same structure as that of the objects in the category we are working with (the structure of a Riemann surface on $U / \operatorname{PSL}(2, \mathbb{Z})$ in our study). So we look for properties of the action of the group that will ensure that the set-theoretic quotient inherits the required structure (in our study $\mathbf{P S L}(2, \mathbb{Z})$ acts properly discontinuously on $U$ and this ensures that the quotient inherits the structure of a Riemann surface from $U)$.

The Theory of Moduli seeks to go beyond mere classification to discover the geometry hidden behind the classification. As mentioned at the end of section 1.4, the core of the problem of moduli lies in analyzing how the structure on the parameter space is connected to or reflects properties of the structure on the objects which were originally sought to be classified. For example, how does the complex structure on $U / \mathbf{P S L}(2, \mathbb{Z}) \cong \mathbb{C}$ relate to the conformal structures on the tori it parametrizes? To make this question precise, we need the notion of a "family"; this notion is described in the next chapter. For motivation, the collection of elliptic curves

$$
\left\{T_{\tau}^{1}=\mathbb{C} / L(\tau) ; \tau \in U\right\}
$$

may be called as a "complex analytic family of elliptic curves parametrized by $U$ " — we will make this precise in (2.2.1.1), (2.2.2.2), (2.4.1.1) and (2.4.2) in chapter 2 . 
Theorem (1.5.3.1) does not indicate the relationship of the structure of Riemann surface on $U / \operatorname{PSL}(2, \mathbb{Z})$ to the Riemann surface structures on the tori it parametrizes. Theorem (4.7.3.5) of chapter 4 captures the beautiful "moduli-theoretic" properties of the Riemann surface $U / \mathbf{P S L}(2, \mathbb{Z})$ namely those properties that relate the holomorphic structure on $U / \mathbf{P S L}(2, \mathbb{Z})$ to "families of holomorphically varying Riemann surface structures on a topological torus". 


\section{Families: Global Deformations}

\subsection{Overview of this Chapter}

This chapter explains the notion of a family of compact complex manifolds whose holomorphic structures depend either differentiably or holomorphically on a variable point of a parameter manifold.

Sections 2.2 and 2.4 respectively define the notions of a differentiable family and of a complex analytic family of compact complex manifolds of fixed dimension. For example, the set of annuli parametrized by the open real interval $(0,1)$, obtained in section 1.4 of the previous chapter, is a differentiable family of (noncompact) complex manifolds. For another example, the set of elliptic curves parametrized by the upper half-plane in complex 1-dimensional space, obtained in section 1.5 of the previous chapter, is a complex analytic family of compact complex manifolds (a holomorphic family of compact Riemann surfaces of genus 1).

Section 2.3 describes the fundamental idea of Kodaira-Spencer, based on which they made precise in [6] the notion of a "variation of complex structures" on a given differentiable manifold. This idea also helps us to naturally define the notion of a "deformation of the complex structure" on a compact complex manifold. The definition of a differentiably varying family of complex structures, given in 2.2, is justified in 2.3.

The notion of equivalence of differentiable families is explained in 2.2 and the corresponding notion for complex analytic families is explained in 2.4. There are many operations on families, like restriction, pullback, etc., which are also explained in these sections. These operations are functorial and lead to the formulation, of a functor of families and hence to a functor of equivalence classes of families, which is made precise in section 2.5. This functorial formulation motivates the general definition of a global moduli problem, which we shall give in chapter 4 . 
In section 2.6, we construct two complex analytic families of complex ndimensional tori which serve as fundamental examples that exhibit various subtle phenomena encountered in many moduli problems. The remarkable local properties of these families are investigated in the next chapter in (3.4.4).

Given a family of compact connected complex manifolds and a fixed point on the parameter space, one is naturally led to ask whether any additional property of the fiber (member of the family) over this point is preserved under sufficiently small variations of its complex structure. For example, this fiber, which is a compact complex manifold, may admit an algebraic structure, say that of a scheme over the field of complex numbers (see (A.4) and (A.5) of the Appendix). Then it is a natural question whether sufficiently close fibers (which represent "small" variations of complex structure of the fixed fiber) also admit algebraic structures. That this question has a negative answer for the case of deformations of the complex structure of a complex $n$-dimensional algebraic torus, for $n \geq 2$, is the content of section 2.7 .

Finally, in section 2.8, we construct an example which shows how the complex structure in a family may suddenly vary at certain points of the parameter space, though it may "remain constant" at all other points in some neighborhood of such a point. To find the causes of such discontinuous variations and to find conditions sufficient to ensure they do not occur, we must study the local properties of families. This leads to the study of "infinitesimal deformations of complex structure" which are explained in the next chapter.

\subsection{Differentiable Families}

In the following we make precise the notion of a differentiable family of compact connected complex manifolds parametrized by a differentiable manifold. By differentiable manifold or simply manifold we mean a connected real differentiable manifold of class $C^{\infty}$. By a map of manifolds we mean a $C^{\infty}$-mapping. The definition we adopt is that of K.Kodaira (see [5], Chap. 4, Sec. 1) who alongwith D.C.Spencer made precise the idea of deformation of complex structure.

\subsubsection{The Definition of a Differentiable Family}

2.2.1.1 Definition. Let $B$ be a domain in $\mathbb{R}^{m}$. For each $t \in B$, let there be given a compact connected complex manifold $M_{t}$ of fixed (complex) dimension $n$. Then the set $\left\{M_{t} ; t \in B\right\}$ of compact connected complex 
manifolds is called a differentiable family of compact connected complex manifolds or is said to be differentiably dependent on the parameter $t \in B$ if there is a surjective map of differentiable manifolds $\phi: \mathcal{M} \longrightarrow B$ such that the following hold:

1. the rank of the Jacobian matrix of $\phi$ is equal to $m$ at each point of $\mathcal{M}$ - in other words, the Jacobian of $\phi$ is of maximal rank everywhere;

2. for each $t \in B, \phi^{-1}(t)$ is a compact connected subset of $\mathcal{M}$;

3. $\phi^{-1}(t)=M_{t}$ and

4. there are a locally finite open covering $\left\{U_{j} ; j \in \mathbb{N}\right\}$ of $\mathcal{M}$ and complexvalued $C^{\infty}$ functions $z_{j}^{1}(p), \ldots, z_{j}^{n}(p)$ defined on $U_{j}$ for each positive integer $j$, such that for each $t$ in $B$ the open cover of $M_{t}$ given by $\left\{U_{j} \cap\right.$ $\left.\phi^{-1}(t) ; U_{j} \cap \phi^{-1}(t) \neq \emptyset\right\}$ forms a system of local complex coordinates for $M_{t}$ with the coordinate map given by $p \mapsto\left(z_{j}^{1}(p), \ldots, z_{j}^{n}(p)\right)$ on each nonempty intersection $U_{j} \cap \phi^{-1}(t)$ in $M_{t}$.

\subsubsection{Notation and Terminology}

We denote the differentiable family $\left\{M_{t} ; t \in B\right\}$ by $(\mathcal{M}, B, \phi)$ or simply by $\mathcal{M}$. $B$ is called the parameter space or the base space and $\mathcal{M}$ the deformation space or the total space associated to this differentiable family.

\subsubsection{Remarks on the Above Definition}

Let $\mathcal{M}$ have dimension $\eta$ as a real differentiable manifold. Then condition (1) of the above definition ensures (by the Implicit Function Theorem) that the fiber of $\phi$ over $t$ viz. $\phi^{-1}(t)$, is a closed embedded real differentiable $s u b$ manifold of $\mathcal{M}$ of dimension $(\eta-m)$ for each $t \in B$. Further, condition (2) of the above definition ensures that the fibers of $\phi$ are compact connected differentiable manifolds. Now the requirement (3) of the above definition is that, for each $t \in B$, the compact connected embedded real differentiable submanifold $\phi^{-1}(t)$ of $\mathcal{M}$ admits a complex structure making it a complex manifold of dimension $n$ biholomorphic to the complex manifold $M_{t}$ prescribed earlier. Therefore, $\eta-m=2 n$.

By using the Implicit Function Theorem for the map $\phi$ we can get a system of local $C^{\infty}$ coordinates $\left\{\left(U_{j}, x_{j}\right) ; j \in \mathbb{N}\right\}$ where $\left\{U_{j} ; j \in \mathbb{N}\right\}$ is a locally finite open cover for $\mathcal{M}$ and $x_{j}: U_{j} \longrightarrow \mathbb{R}^{\eta}$ is the coordinate map given by

$$
x_{j}(p)=\left(x_{j}^{1}(p), \ldots, x_{j}^{2 n}(p), t_{1}, \ldots, t_{m}\right)
$$

and where $\phi(p)=\left(t_{1}, \ldots, t_{m}\right)=t \in B \subset \mathbb{R}^{m}$. In fact, each $x_{j}$ actually maps $U_{j}$ into the product manifold $\mathbb{R}^{2 n} \times B \subset \mathbb{R}^{\eta}$. Further $\phi^{-1}(t)$ as real 
differentiable manifold has a local system of $C^{\infty}$ coordinates given by the open cover $\left\{U_{j} \cap \phi^{-1}(t) ; U_{j} \cap \phi^{-1}(t) \neq \emptyset\right\}$ with the $C^{\infty}$ coordinate map on each nonempty intersection $U_{j} \cap \phi^{-1}(t)$ given by $p \mapsto\left(x_{j}^{1}(p), \ldots, x_{j}^{2 n}(p)\right)$.

Now the purpose of condition (4) of the above definition is to relate the coordinates $\left\{x_{j}^{\alpha} ; 1 \leq \alpha \leq 2 n\right\}$ to the complex structure on $\phi^{-1}(t)$ viz. $M_{t}$ in the following sense: if we set $z_{j}^{\alpha}=x_{j}^{2 \alpha-1}+(\sqrt{-1}) x_{j}^{2 \alpha}$ where $1 \leq \alpha \leq n$, then these are complex-valued functions from $U_{j}$ into $\mathbb{C}^{n}=\mathbb{R}^{2 n}$ which are $C^{\infty}$; the requirement is that (for a suitable choice of the $C^{\infty}$ coordinate system on $\mathcal{M}$ ), the maps

$$
p \mapsto\left(z_{j}^{1}(p), \ldots, z_{j}^{n}(p)\right), p \in \phi^{-1}(t),
$$

serve as complex coordinates for a complex structure on $\phi^{-1}(t)$ isomorphic to $M_{t}$.

We note here that the above definition is easily extended to the case when B is a connected real differentiable manifold.

We further note that we may more generally define in a similar manner a differentiable family of real differentiable compact connected manifolds. Finally, we note that we may define a differentiable family of (not necessarily compact) complex manifolds by removing the word "compact" wherever it occurs in the definition above.

\subsubsection{An Important Note}

The notion of a differentiable family defined above can be formulated in the more sophisticated language of differentiable fiber bundles. This formulation is explained in (3.2.4).

\subsubsection{Examples of Differentiable Families}

The following examples relate to the classification of annuli and tori done in sections 1.4 and 1.5 of chapter 1 .

\subsubsection{Example: Differentiable Family of Annuli}

Set $\mathcal{M}=\left\{(x, y, z) \in \mathbb{R}^{3} ; 0<x<1, x^{2}<y^{2}+z^{2}<1\right\}$. Let $B=(0,1) \subset$ $\mathbb{R}$. Let $\phi: \mathcal{M} \longrightarrow B$ be simply the restriction of the coordinate projection $p_{x}: \mathbb{R}^{3} \longrightarrow \mathbb{R}$ onto the first coordinate. Then

$$
\phi^{-1}\left(x_{0}\right)=\Delta_{x_{0}}:=\left\{w \in \mathbb{C}=\mathbb{R}^{2}=p_{x}^{-1}\left(x_{0}\right) ; x_{0}<|w|<1\right\}
$$

is a complex annulus for each $x_{0} \in B$, where $w=y+(\sqrt{-1}) z$. Then it is clear that $(\mathcal{M}, B, \phi)$ is a differentiable family of complex annuli, where 
we think of this differentiable family only as a family of connected complex manifolds and not as a family of compact connected complex manifolds (cf. (1.4.2)).

\subsubsection{Example: Differentiable Family of Tori}

Let $U$ denote the upper half-plane in $\mathbb{C}$ and $B$ denote the underlying real differentiable manifold of $U$. For each $\tau \in U$, let $T(\tau):=T_{\tau}^{1}$ be the corresponding conformal torus (elliptic curve) as in (1.5.2.2.1). Define the set of mappings

$$
G=\left\{g_{m, n}:(z, w) \mapsto(z+m w+n, w) ; m, n \in \mathbb{Z}, z \in \mathbb{C}, w \in U\right\}
$$

Then $G$ is a group of biholomorphic automorphisms of the product complex manifold $\mathbb{C} \times U$ and acts properly discontinuously and without fixed points. Therefore, $\mathcal{M}:=(\mathbb{C} \times U) / G$ is a complex manifold by (1.5.2.1.2).

The projection $p_{w}: \mathbb{C} \times U \longrightarrow U$ onto the second factor commutes with each element of $G$ and hence gives rise to a holomorphic map $\phi: \mathcal{M} \longrightarrow U$. Then we see that the fiber of $\phi$ over $\tau \in U$ can be canonically identified with the complex torus $T(\tau)=\mathbb{C} / L(\tau)$ where $L=L(\tau)=\{n+m \tau ; n, m \in \mathbb{Z}\}$ is the lattice associated to $\tau$.

The complex coordinates $(z, w)$ on $\mathbb{C} \times U$ give rise to complex coordinates on $\mathcal{M}$. Using these coordinates, it is easy to see that the rank of the complex Jacobian matrix of $\phi$ is equal to 1 . Hence, if we look only at the underlying $C^{\infty}$ structures on $\mathcal{M}$ and $U$, and consider $\phi$ just as a $C^{\infty}$ map, then it is clear that $(\mathcal{M}, B, \phi)$ is a differentiable family of complex tori (cf. (1.5.3.2)).

\subsubsection{Notions of Triviality and Operations on Differentiable Families}

We introduce in the following the notions of equivalence of differentiable families, triviality of a differentiable family, pullback of a differentiable family by a $C^{\infty}$ map, restriction of a differentiable family and local triviality of a differentiable family.

\subsubsection{Equivalence of Differentiable Families}

Suppose that $(\mathcal{M}, B, \phi)$ and $(\mathcal{N}, B, \psi)$ are two differentiable families having the same base space $B$. They are then said to be (differentiably) equivalent if there exists a diffeomorphism $\Phi: \mathcal{M} \longrightarrow \mathcal{N}$ such that for each $t \in$ $B, \Phi$ maps $M_{t}=\phi^{-1}(t)$ biholomorphically onto $N_{t}=\psi^{-1}(t)$. Elementary considerations as in (2.2.1.3) show in such a case that the differentiable family $(\mathcal{N}, B, \psi)$ can be thought of as the differentiable family $(\mathcal{M}, B, \phi)$ 
endowed with a set of new local $C^{\infty}$ coordinates for $\mathcal{M}$. Therefore we often tend to identify differentiably equivalent families.

\subsubsection{Trivial Differentiable Families}

The differentiable family $(M \times B, B, p)$ where $M$ is a compact complex manifold, $B$ is a connected differentiable manifold, $M \times B$ denotes the product differentiable manifold of the underlying differentiable manifold of $M$ and the differentiable manifold $B$ and $p$ is just the projection onto the second factor from this product, is an example of a trivial differentiable family. More generally we say that a differentiable family with base space $B$ is trivial if it is equivalent (in the sense of (2.2.3.1) above) to a differentiable family $(M \times B, B, p)$ for some compact complex manifold $M$. If this happens it is clear that all the fibers (as complex manifolds) are biholomorphic to $M$.

\subsubsection{Induced Differentiable Family: Change of the Parameter}

Suppose that $(\mathcal{M}, B, \phi)$ is a differentiable family of compact connected complex manifolds. Let $h: D \longrightarrow B$ be a $C^{\infty}$ map of connected differentiable manifolds. Define the fiber product

$$
\mathcal{M} \times_{B} D=\{(m, s) \in \mathcal{M} \times D ; \phi(m)=h(s)\},
$$

and consider the image of this fiber product under the canonical map

$$
\phi \times 1_{D}: \mathcal{M} \times D \longrightarrow B \times D
$$

where $1_{D}$ denotes the identity map of $D$. The image is the graph $G_{h}$ of the map $h$. Now $G_{h}$ is naturally a differentiable submanifold of $B \times D$ diffeomorphic to $D$ via the second projection $p$ from the product differentiable manifold $B \times D$.

Since $\phi$ is a $C^{\infty}$ map of maximal rank, so is the map $\phi \times 1_{D}$. Therefore, by the implicit function theorem, the inverse image of the graph of $h$ under the latter map acquires naturally the structure of a differentiable submanifold of $\mathcal{M} \times B$; but this inverse image is precisely the fiber product. Therefore the fiber product becomes, in a natural way, the deformation space of a differentiable family viz. $\left(\mathcal{M} \times_{B} D, D, p \circ\left(\phi \times 1_{D}\right)\right)$.

This differentiable family has parameter space $D$ and is called the family induced from $\mathcal{M}$ via $h$ or the pullback of the family $\mathcal{M}$ by $h$ and is denoted for simplicity by $\left(h^{*} \mathcal{M}, D, h^{*} \phi\right)$.

Note that for each $s \in D$, the complex manifold $\left(h^{*} \phi\right)^{-1}(s)$ is biholomorphic to $M_{h(s)}=\phi^{-1}(h(s))$. Therefore the induced family $\left\{M_{h(s)} ; s \in D\right\}$ 
can be thought of as a family obtained from $\left\{M_{t} ; t \in B\right\}$ by a change of the parameter from $t \in B$ to $s \in D$ via the map $h$ with $h(s)=t$.

It is checked that if $f: E \longrightarrow D$ is a map of connected differentiable manifolds, then the differentiable family on $E$ induced by the composite map $h \circ f$ from the family $\mathcal{M}$ on $B$ can be canonically identified with the differentiable family induced by the map $f$ from the family $h^{*} \mathcal{M}$ on $D$. In other words, the operation of change of parameter is functorial. Further it is clear that the pullback of a trivial family is also a trivial family.

\subsubsection{Restriction of a Differentiable Family}

Let $(\mathcal{M}, B, \phi)$ be a differentiable family. Let $I \subset B$ be a domain in $B$. Thus $I$ acquires in a unique way the structure of an open submanifold of $B$ such that the inclusion map $i: I \hookrightarrow B$ is an open immersion. Then the pullback of the family $(\mathcal{M}, B, \phi)$ by $i$, in the sense of (2.2.3.3) above and denoted as explained there by $\left(i^{*} \mathcal{M}, I, i^{*} \phi\right)$, is called the restriction of $(\mathcal{M}, B, \phi)$ to $I \subset B$. We simplify the notation $\left(i^{*} \mathcal{M}, I, i^{*} \phi\right)$ to $\left(\mathcal{M}_{I}, I, \phi\right)$. The following statements are easily checked: (1) The restriction of a trivial family is trivial. (2) The operation of pulling back commutes with the operation of restriction.

\subsubsection{Locally Trivial Differentiable Families}

A differentiable family $(\mathcal{M}, B, \phi)$ is said to be locally trivial if for each $t \in B$, there is a domain $I \subset B$ containing $t$ such that the restriction of $(\mathcal{M}, B, \phi)$ to $I$ is trivial in the sense of (2.2.3.2). Then the following statements are easy to verify: (1) The pullback of a locally trivial family is also locally trivial. (2) The restriction of a locally trivial family is also locally trivial.

Note that for a locally trivial family, all the fibers are biholomorphically equivalent to each other.

However there exist locally trivial differentiable families that are not (globally) trivial. For such an example, see the construction in (4.8.2) of a family $F$ of elliptic curves which is locally trivial but not globally trivial.

It turns out under certain conditions that locally trivial families correspond precisely to those families which have " no infinitesimal deformations of complex structures" (see (3.2.7.3)). 


\subsection{The Fundamental Idea of Kodaira-Spencer}

\subsubsection{The Local Triviality of Differentiable Families}

Recall each of the examples $(\mathcal{M}, B, \phi)$ of (2.2.2). As already noted in the beginnings of (1.4) and (1.5.1), we see that all the fibers of $\phi$ are isomorphic as real differentiable manifolds. Therefore we can think of each of these families as parametrizing varying complex structures on the same real differentiable manifold.

It turns out in general that for any differentiable family of compact connected complex manifolds $(\mathcal{M}, B, \phi)$, all the fibers of $\phi$ are diffeomorphic to one another.

Therefore we may think of a differentiable family $(\mathcal{M}, B, \phi)$ as a family of varying complex structures on the same underlying differentiable manifold.

The basic theorem that implies this is the following. For its proof, we refer to [5], Chap. 2, Theorem 2.4.

2.3.1.1 Theorem. Let $(\mathcal{M}, B, \phi)$ be a differentiable family of compact connected real differentiable manifolds. Then for each $t_{0} \in B$, there is a $C^{\infty}$ coordinate neighborhood $U$ of $t_{0}$ such that the restriction $\left(\mathcal{M}_{U}, U, \phi\right)$ of $(\mathcal{M}, B, \phi)$ to $U$ is equivalent to the trivial family $\left(M_{t_{0}} \times U, U, p\right)$ where $M_{t_{0}}=\phi^{-1}\left(t_{0}\right)$ and $p$ from $M_{t_{0}} \times U$ onto $U$ is the coordinate projection onto the second factor. In other words, a differentiable family of real compact connected differentiable manifolds is locally trivial.

2.3.1.2 Remark. The above theorem may be stated as follows in the sophisticated language of fiber bundles.

A differentiable family of compact connected real differentiable manifolds is a locally trivial differentiable fiber bundle.

This version of the above theorem is the starting point for another formulation of the notion of a differentiable family — see (3.2.4).

\subsection{2 $C^{\infty}$-Deformations of Complex Structure}

Now we come to the fundamental and fruitful idea of Kunihiko Kodaira and D.C.Spencer which is the basis of their Theory of Deformation of Complex Structures (see [6]). According to them, a deformation of the complex structure on a complex manifold $M$ is another complex structure on the underlying differentiable manifold of $M$ obtained by using the same local complex coordinate charts on $M$ but with different glueing (or transition) functions. To understand this notion, we first recall below how a complex manifold $M$ of (complex) dimension $n$ is obtained. 
2.3.2.1 Definition. Choose a finite or countable open cover $\left\{U_{i}\right\}$ of a connected second-countable locally-Euclidean Hausdorff topological space $M$ and choose for each $i$, a homeomorphism $z_{i}: U_{i} \longrightarrow \mathbb{C}^{n}$ onto a domain $V_{i}$ in $\mathbb{C}^{n}$. Now transport the natural complex structure on the domain $V_{i}=z_{i}\left(U_{i}\right) \subset \mathbb{C}^{n}$ to $U_{i}$ via $z_{i}$ so that $z_{i}$ becomes a holomorphic map. We call each pair $\left(U_{i}, z_{i}\right)$ a local complex coordinate chart, $U_{i}$ the associated local complex coordinate neighborhood and $z_{i}$ the associated local complex coordinate. We denote the intersection of $U_{j}$ and $U_{k}$ as $U_{j k}$ and denote by $V_{k j}$ the image of $U_{k j}$ under $z_{k}$. Clearly, whenever $U_{j k}$ is nonempty, $U_{j k}$ has two complex structures, one each arising from the coordinates $z_{j}$ and $z_{k}$. We now require that these two complex structures on $U_{j k}$ be compatible (or that $U_{j}$ and $U_{k}$ be glued biholomorphically along $U_{j k}$ ) by imposing the condition that the homeomorphism

$$
f_{j k}:=z_{j} \circ z_{k}^{-1}: V_{k j} \longrightarrow V_{j k}
$$

of domains in $\mathbb{C}^{n}$ be biholomorphic for each nonempty intersection $U_{k j}$. The biholomorphic maps $f_{j k}$ are called transition functions or glueing functions and they satisfy the patching condition $f_{j k}=f_{j l} \circ f_{l k}$. With all the above conditions being satisfied, the collection of charts $\left\{\left(U_{i}, z_{i}\right)\right\}$ is called a system of local complex coordinates for $M$ and $M$ together with such a maximal system is called a complex manifold of dimension $n$.

By a morphism (or holomorphic map) of complex manifolds is meant a continuous map which induces holomorphic maps locally via the local coordinates.

We may assume that the open cover $\left\{U_{i}\right\}$ is locally finite and also that each domain $V_{i} \subset \mathbb{C}^{n}$ is a unit polydisc $V_{i}=\left\{z_{i}(p)=\left(z_{i}^{1}(p), \ldots, z_{i}^{n}(p)\right) \in\right.$ $\left.\mathbb{C}^{n} ; p \in U_{i},\left|z_{i}^{\alpha}(p)\right|<1, \alpha=1, \ldots, n\right\}$. Notice that $\left(z_{j}^{1}, \ldots, z_{j}^{n}\right) \in V_{j}$ and $\left(z_{k}^{1}, \ldots, z_{k}^{n}\right) \in V_{k}$ represent the same point of $M$ if and only if

$$
\left(z_{j}^{1}, \ldots, z_{j}^{n}\right)=f_{j k}\left(z_{k}^{1}, \ldots, z_{k}^{n}\right)
$$

which is written coordinatewise as

$$
z_{j}^{\alpha}=f_{j k}^{\alpha}\left(z_{k}\right)=f_{j k}^{\alpha}\left(z_{k}^{1}, \ldots, z_{k}^{n}\right) \text { for each } \alpha=1, \ldots, n .
$$

2.3.2.2 Remark. In the above definition, if we replace $\mathbb{C}^{n}$ by $\mathbb{R}^{n}$ and replace all holomorphic maps by $C^{\infty}$-maps, then we obtain the definition of a real differentiable manifold of dimension $n$. With similar modifications, one may define a real differentiable manifold of class $C^{k}$ where $k$ is a positive integer and also a locally Euclidean manifold.

It would be instructive for the interested reader to check that these definitions are equivalent to the ones given in (A.5). 


\subsubsection{Deformations of a Complex Manifold}

If $M$ is a compact complex manifold of dimension $n$ as defined above, then we may assume that its system of local complex coordinates is finite: $\left\{\left(U_{i}, z_{i}\right) ; i=1, \ldots, l\right\}$. Thus a compact complex manifold $M$ is obtained by glueing a finite number of polydiscs $V_{1}, \ldots, V_{l}$ i.e., by identifying $z_{k} \in V_{k}$ and $z_{j}=f_{j k}\left(z_{k}\right) \in V_{j}$.

A deformation of $M$ is considered to be the complex manifold obtained by glueing the same polydiscs $V_{1}, \ldots, V_{l}$ via different transition functions. In other words, we replace $f_{j k}^{\alpha}\left(z_{k}\right)$ by the functions

$$
f_{j k}^{\alpha}\left(z_{k}, t\right)=f_{j k}^{\alpha}\left(z_{k}, t_{1}, \ldots, t_{m}\right)
$$

with the initial conditions $f_{j k}^{\alpha}\left(z_{k}, t_{0}\right)=f_{j k}^{\alpha}\left(z_{k}\right)$ where $t=\left(t_{1}, \ldots, t_{m}\right)$ is a parameter which represents a variable point on some (complex or real differentiable) connected manifold $B$. The initial conditions ensure that for $t=t_{0} \in B$, the deformation that we obtain is $M$ itself. Further, for each $t \in B$, if we denote by $M_{t}$ the complex manifold obtained by glueing the polydiscs $V_{1}, \ldots, V_{l}$ via the transition functions $f_{j k}\left(z_{k}, t\right)$, then each $M_{t}$ is called a deformation of $M_{t_{0}}=M$. This is the fundamental idea of K.Kodaira and D.C. Spencer.

It then happens that if we consider a differentiable family $(\mathcal{M}, B, \phi)$ of compact connected complex manifolds $M_{t}=\phi^{-1}(t), t \in B$, then for a fixed fiber $M_{t_{0}}=\phi^{-1}\left(t_{0}\right), t_{0} \in B$, any other sufficiently close fiber $M_{t}=\phi^{-1}(t)$ (i.e., with $t$ sufficiently close to $t_{0}$ ) is simply a complex manifold obtained by glueing the same polydiscs that form a system of local complex coordinate neighborhoods for $M$ but by different transition functions, in exactly the way mentioned above, so that the nearby fibers are actually deformations of $M$. Further, by applying theorem (2.3.1.1), we see that all the fibers are diffeomorphic to each other.

Hence the notion of a differentiable family indeed captures locally the idea of a $C^{\infty}$ - deformation of complex structures on a fixed differentiable manifold.

We precisely formulate and prove the exact relationship between deformations and differentiable families in the following.

2.3.2.4 Theorem (Nearby fibers of a family are deformations of each other). $\quad \operatorname{Let}(\mathcal{M}, B, \phi)$ be a differentiable family of compact connected complex manifolds. Fix a point $t_{0} \in B$ and let $M_{t_{0}}=\phi^{-1}\left(t_{0}\right)$ be the compact connected complex manifold obtained by glueing the polydiscs $V_{1}, \ldots, V_{l}$ by identifying $z_{k} \in V_{k}$ and $z_{j}=f_{j k}\left(z_{k}\right) \in V_{j}$ via the transition functions $\left\{f_{j k}\right\}$ arising out of a given finite system of local complex coordinates $\left\{\left(U_{i}, z_{i}\right) ; i=1, \ldots, l\right\}$ i.e., $z_{j}\left(U_{j}\right)=V_{j}$ and $f_{j k}=z_{j} \circ z_{k}^{-1}$. 
Then there exists a domain $I \subset B$ containing $t_{0}$ such that for each $t \in$ $I, M_{t}=\phi^{-1}(t)$ is a compact complex manifold obtained by glueing the same polydiscs $V_{1}, \ldots, V_{l}$ but by different transition functions

$$
f_{j k}^{\alpha}\left(z_{k}, t\right)=f_{j k}^{\alpha}\left(z_{k}, t_{1}, \ldots, t_{m}\right)
$$

with the initial conditions $f_{j k}^{\alpha}\left(z_{k}, t_{0}\right)=f_{j k}^{\alpha}\left(z_{k}\right)$ where $t=\left(t_{1}, \ldots, t_{m}\right)$ are local $C^{\infty}$ coordinates on $I$ and the functions $f_{j k}\left(z_{k}, t\right)$ are $C^{\infty}$ functions of $t$.

Proof. Given a differentiable family $(\mathcal{M}, B, \phi)$, as explained in (2.2.1.3) we can find a locally finite open cover $\left\{U_{j}^{\prime} ; j \in \mathbb{N}\right\}$ of $\mathcal{M}$ and a system of $C^{\infty}$ local coordinate charts $\left\{\left(U_{j}^{\prime}, x_{j}\right) ; j \in \mathbb{N}\right\}$ with each $x_{j}$ a diffeomorphism of $U_{j}^{\prime}$ onto $V_{j} \times I_{j}$ where $V_{j} \subset \mathbb{R}^{2 n}=\mathbb{C}^{n}$ is a domain that can be assumed to be a polydisc:

$V_{j}=\left\{z_{j}(p)=\left(z_{j}^{1}(p), \ldots, z_{j}^{n}(p)\right) \in \mathbb{C}^{n} ; p \in U_{j}^{\prime},\left|z_{j}^{\alpha}(p)\right|<r_{j}^{\alpha}, \alpha=1, \ldots, n\right\}$

and where $I_{j} \subset B$ is (identified via a local $C^{\infty}$ coordinate map with) a domain in $\mathbb{R}^{m}, m$ being the dimension of $B$ as a real differentiable manifold. Here $I_{j}$ can be chosen to be an open multi-interval given by

$$
I_{j}=\left\{t=\left(t_{1}, \ldots, t_{m}\right) \in \mathbb{R}^{m} ; a_{j \nu}<t_{\nu}<b_{j \nu}, \nu=1, \ldots, m\right\} .
$$

Thus we have for $p \in U_{j}^{\prime} \subset \mathcal{M}$,

$$
x_{j}(p)=\left(z_{j}^{1}(p), \ldots, z_{j}^{n}(p), t_{1}, \ldots, t_{m}\right), \phi(p)=\left(t_{1}, \ldots, t_{m}\right) .
$$

The transition functions $\left\{f_{j k}\right\}$ which glue the domains $\left\{V_{j} \times I_{j}\right\}$ to give the differentiable manifold $\mathcal{M}$ are given as usual by $f_{j k}=x_{j} \circ x_{k}^{-1}$ and are $C^{\infty}$ functions of the variables $t$ and $z_{k}$ (in fact, the functions $f_{j k}$ are holomorphic in the variables $\left.z_{k}\right)$ and hence are written as $f_{j k}\left(z_{k}, t\right)$. Thus the points $\left(z_{j}, t\right) \in V_{j} \times I_{j}$ and $\left(z_{k}, t^{\prime}\right) \in V_{k} \times I_{k}$ represent the same point of $\mathcal{M}$ if and only if $\left(z_{j}, t\right)=f_{j k}\left(z_{k}, t^{\prime}\right)$ which also means that $t$ and $t^{\prime}$ must represent the same point of $B$.

Now fix any $t_{0} \in B$. Then $M_{t_{0}}=\phi^{-1}\left(t_{0}\right)$ is the compact connected complex manifold obtained by glueing the polydiscs $\left\{V_{j} \times\left\{t_{0}\right\} \cong V_{j} ; U_{j}^{\prime} \cap\right.$ $\left.M_{t_{0}} \neq \emptyset\right\}$ via the (biholomorphic!) transition functions $\left\{f_{j k}\left(z_{k}, t_{0}\right)\right\}$ and therefore the points $z_{j} \in V_{j}$ and $z_{k} \in V_{k}$ represent the same point of $M_{t_{0}}=\phi^{-1}\left(t_{0}\right)$ if and only if $z_{j}=f_{j k}\left(z_{k}, t_{0}\right)$.

By the compactness of the fibers of $\phi$ and the fact that the open cover $\left\{U_{j}^{\prime}\right\}$ is locally finite, it is easy to see that there exists an open neighborhood $I$ of $t_{0}$ in $B$ (which can be identified with an open multi-interval in $\mathbb{R}^{m}$ via local coordinates at $t_{0}$ ) such that $\phi^{-1}(I)$ is contained in the union of a finite 
number of $U_{j}^{\prime}$ which we assume without loss of generality to be $U_{1}^{\prime}, \ldots, U_{l}^{\prime}$. Let $U_{j}=U_{j}^{\prime} \cap M_{t_{0}}$ for each $j$. Now let $t \in I$.

Then by construction, $M_{t}$ is a compact connected complex manifold obtained by glueing the polydiscs $\left\{V_{j} \times\{t\} \cong V_{j} ; j=1, \ldots, l\right\}$ via the transition functions $\left\{f_{j k}\left(z_{k}, t\right)\right\}$ (which depend holomorphically on the variables $z_{k}$ ) and therefore the points $z_{j} \in V_{j}$ and $z_{k} \in V_{k}$ represent the same point of $M_{t}=\phi^{-1}(t)$ if and only if $z_{j}=f_{j k}\left(z_{k}, t\right)$.

Therefore we see that the restriction of $(\mathcal{M}, B, \phi)$ to $I \subset B$, which gives the differentiable family $\left(\mathcal{M}_{I}, I, \phi\right)$ as in (2.2.3.4), consists of fibers which are all compact connected complex manifolds obtained by glueing the same polydiscs $\left\{V_{1}, \ldots, V_{l}\right\}$ but with transition functions $f_{j k}\left(z_{k}, t\right)$ which depend differentiably on the parameter $t \in I$.

We see that these polydiscs themselves are independent of the parameter $t \in I$ and only the way in which they are glued together is dependent on $t \in I$ so as to determine various deformations of the complex structure on any (chosen) fixed fiber $M_{t_{0}}=\phi^{-1}\left(t_{0}\right), t_{0} \in I \subset B$ QED

Motivated by the above theorem, we make the following definitions.

2.3.2.5 Definitions (Local and Global Deformations). Let $M$ be a compact connected complex manifold and $(\mathcal{M}, B, \phi)$ a differentiable family of compact connected complex manifolds with $M \cong M_{t_{0}}=\phi^{-1}\left(t_{0}\right)$ for some $t_{0} \in B$. Then there exists a domain $I$ in $B$ containing $t_{0}$ which satisfies the conditions of the above theorem. We call the compact connected complex manifold $M_{t}=\phi^{-1}(t)$, for each $t \in I$, a local $C^{\infty}$-deformation of complex structure of $M$. More generally we call the fiber $M_{t}=\phi^{-1}(t)$, for each $t \in B$, a (global) $C^{\infty}$-deformation of complex structure of $M$.

The differentiable manifold $\mathcal{M}$ is called a total space of (global) $C^{\infty}$ deformations of complex structure of $M$ and $B$ is called the parameter space or the base space. Recall that the underlying structure of differentiable manifold does not change under deformation as explained in theorem (2.3.1.1). So, by studying existence and properties of deformation spaces, we are actually studying the relationship of the variation of complex structure on the underlying differentiable manifold of a fixed compact complex manifold to the properties and structure of the corresponding parameter spaces.

It is clear from the theorem (2.3.2.4) that a sufficiently small local complex coordinate neighborhood of each point of a fixed compact complex manifold does not change its holomorphic structure when deformed locally.

\subsubsection{A Preliminary Formulation of the Problem of Moduli}

In the following we adopt the definitions given by C.S.Seshadri in his survey article [7]. 
Given a compact connected complex manifold $M$, by the problem of moduli of $M$ we mean an investigation of the set $X$ of isomorphism classes of global deformations of $M$ to answer the following questions:

(a) whether a suitable geometric structure exists on this set $X$ (for e.g., whether $X$ admits the structure of a $C^{\infty}$ manifold, complex manifold, complex analytic space or algebraic scheme);

(b) whether special properties are found to be satisfied by the structures obtained on $X$ in (a) above, particularly those that relate to properties intrinsic to $M$.

The problem of moduli of $M$ can be formally divided into two subproblems:

1. the problem of local moduli of $M$;

2. the problem of global moduli of $M$.

The problem of local moduli may be stated to be the study of the infinitesimal neighborhoods of the point of $X$ corresponding to (the isomorphism class of the trivial deformation of) $M$, provided $X$ has some decent structure, say differentiable, holomorphic, analytic or algebraic. For example, if $x_{0} \in X$ corresponds to $M$, then such a study will involve the computation of the (analytic or algebraic) tangent space of $X$ at $x_{0}$, a computation of the dimension of $X$ at $x_{0}$ and also an investigation as to whether $X$ is smooth or singular at $x_{0}$. The Theory of Local Moduli is also called the study of infinitesimal deformations and is the main theme of the next chapter. The best solution that one can hope to obtain for a local moduli problem is called a "local moduli space" — this is explained in the next chapter (see (3.6)).

The problem of global moduli of $M$ involves the investigation of global properties of the structure on $X$, for example, if $X$ itself is a parameter space of some family of deformations $(\mathcal{M}, X, \phi)$ of $M$ possessing some special properties with respect to other families which consist of deformations of $M$. The notion of a global moduli problem is precisely formulated in functorial terms in chapter 4 (see (4.5)). The best solution that one can hope to obtain for a global moduli problem is called a "fine moduli space", while a less better solution that one often gets is called a "coarse moduli space" these concepts are explained in chapter 4 (see (4.5)).

\subsection{Complex Analytic Families}

In section 2.2, we defined the notion of a differentiably varying family of compact connected complex manifolds parametrized by a differentiable 
manifold. In this section, we make precise the corresponding notion for the category of complex manifolds and holomorphic maps i.e., the notion of a holomorphically varying family of compact connected complex manifolds parametrized by a complex connected manifold. The following definition is an analogue of (2.2.1.1) and is again due to Kodaira-Spencer [6].

\subsubsection{The Definition of a Complex Analytic Family}

2.4.1.1 Definition. Let $B$ be a domain in $\mathbb{C}^{m}$. For each $t \in B$, let there be given a compact connected complex manifold $M_{t}$ of fixed (complex) dimension $n$. Then the set $\left\{M_{t} ; t \in B\right\}$ of compact connected complex manifolds is called a complex analytic family of compact connected complex manifolds or is said to be holomorphically dependent on the parameter $t \in B$ if there is a surjective holomorphic map of complex manifolds $\phi: \mathcal{M} \longrightarrow B$ such that the following hold:

1. the rank of the Jacobian matrix of $\phi$ is equal to $m$ at each point of $\mathcal{M}$ - in other words, the Jacobian of $\phi$ is of maximal rank everywhere;

2. for each $t \in B, \phi^{-1}(t)$ is a compact connected subset of $\mathcal{M}$;

3. $\phi^{-1}(t)=M_{t}$.

\subsubsection{Notation and Terminology}

We denote the complex analytic family $\left\{M_{t} ; t \in B\right\}$ by $(\mathcal{M}, B, \phi)$ or simply by $\mathcal{M} . B$ is called the parameter (or base) space and $\mathcal{M}$ the deformation (or total) space associated to this complex analytic family.

\subsubsection{Remarks on the Above Definition}

Let $\mathcal{M}$ have dimension $\eta$ as a complex manifold. Then condition (1) of the above definition ensures (by the Implicit Function Theorem) that the fiber of $\phi$ over $t$ viz. $\phi^{-1}(t)$, is a closed embedded complex submanifold of $\mathcal{M}$ of dimension $(\eta-m)$ for each $t \in B$. Further, (2) of the above definition ensures that the fibers are compact connected complex manifolds. Now the condition (3) requires for each $t \in B$ that the compact connected embedded complex submanifold $\phi^{-1}(t)$ of $\mathcal{M}$ is biholomorphic to the complex manifold $M_{t}$ prescribed earlier. Therefore, $\eta-m=n$.

In the statement of condition (1) of the above definition, by the Jacobian of $\phi$ we mean the Jacobian matrix of the holomorphic map $\phi$ written in terms of local complex coordinates for $\mathcal{M}$ and $B$.

By using the Implicit Function theorem for the map $\phi$ we can get a system of local complex coordinates $\left\{\left(U_{j}, z_{j}\right) ; J \in \mathbb{N}\right\}$ where $\left\{U_{j} ; j \in \mathbb{N}\right\}$ is 
a locally finite open cover for $\mathcal{M}$ and $z_{j}: U_{j} \longrightarrow \mathbb{C}^{\eta}$ is the holomorphic complex coordinate map onto a polydisc $V_{j} \subset \mathbb{C}^{n+m}$ such that

$$
z_{j}(p)=\left(z_{j}^{1}(p), \ldots, z_{j}^{n}(p), t_{1}, \ldots, t_{m}\right)
$$

and where $\phi(p)=\left(t_{1}, \ldots, t_{m}\right)=t \in B \subset \mathbb{C}^{m}$. In fact, each $z_{j}$ actually maps $U_{j}$ into the product complex manifold $\mathbb{C}^{n} \times B \subset \mathbb{C}^{\eta}$. Further, $\phi^{-1}(t)=M_{t}$, for each $t \in B$ (as complex manifold) has a system of local complex coordinates given by the open cover $\left\{U_{j} \cap \phi^{-1}(t) ; U_{j} \cap \phi^{-1}(t) \neq \emptyset\right\}$ with the local complex coordinate map on each nonempty intersection $U_{j} \cap \phi^{-1}(t)$ given by $p \mapsto\left(z_{j}^{1}(p), \ldots, z_{j}^{n}(p)\right)$. In terms of these local complex coordinates, $\phi$ is simply the projection given by

$$
\left(z_{j}^{1}, \ldots, z_{j}^{n}, t_{1}, \ldots, t_{m}\right) \mapsto\left(t_{1}, \ldots, t_{m}\right) .
$$

The polydiscs $\left\{V_{j} ; j \in \mathbb{N}\right\}$ are glued by the holomorphic transition functions $\left\{f_{j k} ; j, k \in \mathbb{N}\right\}$ where $f_{j k}=z_{j} \circ z_{k}^{-1}$ for each nonempty intersection $U_{j} \cap U_{k}$. Then $f_{j k}$ is a holomorphic function of the variables $t$ and $z_{k}$ and is hence written coordinatewise as

$$
f_{j k}^{\alpha}\left(z_{k}, t\right)=f_{j k}^{\alpha}\left(z_{k}^{1}, \ldots, z_{k}^{n}, t_{1}, \ldots, t_{m}\right)
$$

for each $\alpha=1, \ldots, \eta$. Thus for $t_{0} \in B, M_{t_{0}}$ is a compact connected complex manifold obtained by glueing the polydiscs

$$
\left\{V_{j, t_{0}}:=p^{-1}\left(t_{0}\right) \cap V_{j} ; j \in \mathbb{N}\right\}
$$

in $\mathbb{C}^{n}$ (where $p$ is the canonical projection onto the second factor from the product complex manifold $\mathbb{C}^{n} \times B$ ) via the holomorphic transition functions $\left\{f_{j k}\left(z_{k}, t_{0}\right) ; j, k \in \mathbb{N}\right\}$ so that the point $z_{j} \in V_{j, t_{0}}$ represents the same point of $M_{t_{0}}$ as the point $z_{k} \in V_{k, t_{0}}$ if and only if $z_{j}=f_{j k}\left(z_{k}, t_{0}\right)$.

The above definition is easily extended to the case when $B$ is a connected complex manifold.

It is also clear that one may define a complex analytic family of (not necessarily compact) complex manifolds by removing the word "compact" wherever it occurs in the definition above. Finally we note that, given a complex analytic family $(\mathcal{M}, B, \phi)$, by considering only the underlying differentiable manifold structures on $\mathcal{M}$ and $B$ and by regarding the map $\phi$ just as a $C^{\infty}$ map, we see that we obtain a differentiable family of compact connected complex manifolds which we refer to as the underlying differentiable family of the complex analytic family $(\mathcal{M}, B, \phi)$. We denote this underlying differentiable family also by $(\mathcal{M}, B, \phi)$, leaving the context of the discussion to indicate that we are interested only in the underlying differentiable family. 


\subsubsection{Examples of Complex Analytic Families}

We recall example (2.2.2.2) where we described the differentiable family $(\mathcal{M}, B, \phi)$ of complex tori parametrized by the upper half-plane $U$; here $B$ is the underlying differentiable manifold of $U$. From the calculations there it is evident now that $(\mathcal{M}, U, \phi)$ is actually a complex analytic family of complex tori parametrized by $U$.

For interesting generalizations of the above complex analytic family to higher dimensions refer to (2.6.1) and (2.6.2). Another interesting example is given by the family of Hopf surfaces constructed in (2.8.2).

\subsubsection{Notions of Triviality and Operations on Complex Analytic Families}

Just as in the case of differentiable families, we next define the notions of equivalence, triviality, pullback, restriction and local triviality for complex analytic families (cf. (2.2.3)).

\subsubsection{Holomorphic Equivalence of Complex Analytic Families}

Suppose $(\mathcal{M}, B, \phi)$ and $(\mathcal{N}, B, \psi)$ are two complex analytic families. Then they are said to be holomorphically equivalent if there exists a biholomorphic map $\Phi: \mathcal{M} \longrightarrow \mathcal{N}$ such that for each $t \in B, \Phi$ maps $M_{t}=\phi^{-1}(t)$ biholomorphically onto $N_{t}=\psi^{-1}(t)$. Elementary considerations show in such a case that the complex analytic family $(\mathcal{N}, B, \psi)$ can be thought of as the complex analytic family $(\mathcal{M}, B, \phi)$ endowed with a set of new local complex coordinates for $\mathcal{M}$. Therefore we often tend to identify equivalent families.

\subsubsection{Trivial Complex Analytic Family}

The complex analytic family $(M \times B, B, p)$ where $M$ is a compact complex manifold, $B$ is a connected complex manifold, $M \times B$ denotes the product complex manifold of $M$ and $B$, and $p$ is just the projection onto the second factor from this product, is an example of a trivial complex analytic family. More generally, we say that a complex analytic family is trivial if it is equivalent (in the sense of (2.4.3.1)) to a complex analytic family $(M \times B, B, p)$ as above where $M$ is some compact complex manifold. It is clear that in this case all the fibers (as complex manifolds) are biholomorphic to $M$.

It is obvious that if a complex analytic family is trivial, then the underlying differentiable family is also trivial.

It turns out that the converse of the above assertion is also true but this is nontrivial to prove (see (3.2.9.2)). 


\subsubsection{Induced Complex Analytic Family: Change of the Parameter}

Suppose $(\mathcal{M}, B, \phi)$ is a complex analytic family of compact connected complex manifolds. Let $h: D \longrightarrow B$ be a holomorphic map of connected complex manifolds. Define the fiber product

$$
\mathcal{M} \times_{B} D=\{(m, s) \in \mathcal{M} \times D ; \quad \phi(m)=h(s)\},
$$

and consider the image of this fiber product under the canonical map

$$
\phi \times 1_{D}: \mathcal{M} \times D \longrightarrow B \times D
$$

where $1_{D}$ denotes the identity map of $D$. The image is the graph $G_{h}$ of the map $h$. Now $G_{h}$ is naturally a complex submanifold of $B \times D$ and is biholomorphic to $D$ via the second projection $p$ from the product complex manifold $B \times D$.

Since $\phi$ is a holomorphic map of maximal rank, so is the map $\phi \times 1_{D}$. Therefore, by the Implicit Function theorem, the inverse image of the graph of $h$ under the latter map acquires naturally the structure of a complex submanifold of $\mathcal{M} \times B$; but this inverse image is precisely the fiber product. Therefore the fiber product becomes, in a natural way, the deformation space of a complex analytic family viz. $\left(\mathcal{M} \times_{B} D, D, p \circ\left(\phi \times 1_{D}\right)\right)$.

This complex analytic family has parameter space $D$ and is called the family induced from $\mathcal{M}$ via $h$ or the pullback of the family $\mathcal{M}$ by $h$ and is denoted for simplicity by $\left(h^{*} \mathcal{M}, D, h^{*} \phi\right)$.

Note that for each $s \in D$, the complex manifold $\left(h^{*} \phi\right)^{-1}(s)$ is biholomorphic to $M_{h(s)}=\phi^{-1}(h(s))$. Therefore the induced family $\left\{M_{h(s)} ; s \in D\right\}$ can be thought of as a family obtained from $\left\{M_{t} ; t \in B\right\}$ by a change of the parameter from $t \in B$ to $s \in D$ via the map $h$ with $h(s)=t$.

It is clear that if $f: E \longrightarrow D$ is a map of connected complex manifolds, then the complex analytic family on $E$ induced by the composite map $h \circ f$ from the family $\mathcal{M}$ on $B$, can be canonically identified with the complex analytic family induced by the map $f$ from the family $h^{*} \mathcal{M}$ on $D$. Thus the operation of change of parameter is functorial. Further it is clear that the pullback of a trivial family is also a trivial family.

\subsubsection{Restriction of a Complex Analytic Family}

Let $(\mathcal{M}, B, \phi)$ be a complex analytic family. Let $I \subset B$ be a domain in $B$. Thus $I$ acquires in a unique way the structure of an open submanifold of $B$ such that the inclusion map $i: I \hookrightarrow B$ is an open immersion. Then the pullback of the family $(\mathcal{M}, B, \phi)$ by $i$, in the sense of (2.4.3.3) above and denoted as explained there by $\left(i^{*} \mathcal{M}, I, i^{*} \phi\right)$, is called the restriction of 
$(\mathcal{M}, B, \phi)$ to $I \subset B$. Further, instead of writing $\left(i^{*} \mathcal{M}, I, i^{*} \phi\right)$ we shall write $\left(\mathcal{M}_{I}, I, \phi\right)$. The following statements are then obvious: (1) The restriction of a trivial family is trivial. (2) The operation of pulling back commutes with the operation of restriction.

\subsubsection{Locally Trivial Complex Analytic Family}

A complex analytic family $(\mathcal{M}, B, \phi)$ is said to be locally trivial if for each $t \in B$, there is a domain $I \subset B$ containing $t$ such that the restriction of $(\mathcal{M}, B, \phi)$ to $I$ is trivial in the sense of (2.4.3.2).

It is clear that the pullback of a locally trivial family is also locally trivial.

Under certain conditions, locally trivial families correspond precisely to those families which have "no infinitesimal deformations of complex structures" (cf. (2.2.3.5 and 2.4.3.2)).

Note that for a locally trivial family, all the fibers are biholomorphically equivalent to each other. However there exist locally trivial holomorphic families that are not (globally) trivial (see (4.8.2)). The following statement will be proved in (3.2.9.1).

A complex analytic family is locally trivial if and only if it is locally trivial as a differentiable family.

\subsubsection{Remarks on Holomorphic Deformations of Complex Structure}

Let $(\mathcal{M}, B, \phi)$ be a complex analytic family of compact connected complex manifolds. By the discussion in (2.3) it is clear that the underlying differentiable manifold structure of each of the fibers $M_{t}=\phi^{-1}(t)$ is the same (up to diffeomorphism). It is also clear from (2.3.2.4) that for a fixed fiber $M_{t_{0}}=\phi^{-1}\left(t_{0}\right), t_{0} \in B$, any other sufficiently close fiber $M_{t}=\phi^{-1}(t)$ is simply a complex manifold obtained by glueing the same polydiscs that form a system of local complex coordinate neighborhoods for $M_{t_{0}}$ but by different transition functions which depend holomorphically on $t \in B, t$ sufficiently close to $t_{0}$.

Hence the notion of a complex analytic family indeed captures locally the idea of a holomorphic deformation of complex structures on a fixed differentiable manifold.

Just as in (2.3.2.5), by considering complex analytic families instead of differentiable families, we may define the notions of a local holomorphic deformation and a global holomorphic deformation of the complex structure of a fixed compact complex manifold, and we may also formulate (as in (2.3.2.6)), the problems of local and global moduli for such holomorphic 
deformations.

In the next section, we describe the functorial properties of families. This functorial viewpoint is fundamental to the "Theory of Moduli" where problems are often formulated in functorial terms.

\subsection{Functorial Properties of Families}

Let $M$ be a compact connected complex manifold. Let $M_{C^{\infty}}$ denote the underlying differentiable manifold of $M$. We wish to study those structures of complex manifold that can be imposed on $M_{C^{\infty}}$ which also occur as deformations of the complex structure $M$ on $M_{C^{\infty}}$.

Let $S$ denote the set of pairs of the form $\left(M_{C^{\infty}},\left\{\left(U_{j}, z_{j}\right) ; j \in \mathbb{N}\right\}\right)$ where $\left\{\left(U_{j}, z_{j}\right) ; j \in \mathbb{N}\right\}$ is a system of local complex coordinates on $M_{C^{\infty}}$ that endows $M_{C} \infty$ with the structure of a complex manifold which occurs as a deformation of $M$.

We define the equivalence relation $\sim_{S}$ on $S$ by declaring two elements of $S$ to be equivalent if and only if they give rise to biholomorphic complex structures on $M_{C}$. Thus, the set-theoretic quotient $S / \sim_{S}$ of $S$ by $\sim_{S}$ is simply the set of conformal isomorphism classes of complex manifold structures on $M_{C^{\infty}}$ that occur as deformations of $M$.

The following discussion applies to both complex analytic families and differentiable families. Therefore in the following, the words "family", "deformation", "manifold", "map of manifolds" could be replaced throughout by the words "complex analytic family", "holomorphic deformation", "complex manifold", "holomorphic map" respectively; or throughout by the words "differentiable family", " $C^{\infty}$ deformation", "differentiable manifold", " $C^{\infty}$ map" respectively.

\subsubsection{The Functor of Families}

Let $\mathcal{C}$ denote the category whose objects are pairs of the form $\left(B, t_{0}\right)$ where $B$ is a connected manifold and $t_{0}$ a point of $B$ called the base point; morphisms in $\mathcal{C}$ are maps of manifolds that preserve base points i.e., a morphism from $\left(B^{\prime}, t_{0}^{\prime}\right)$ to $\left(B, t_{0}\right)$ is a map of manifolds $f: B^{\prime} \longrightarrow B$ which maps $t_{0}^{\prime}$ onto $t_{0}$.

Let $\mathcal{S}$ denote the category whose objects are sets and whose morphisms are set-theoretic maps. Define the association $\mathcal{F} \mathcal{A M}: \mathcal{C} \longrightarrow \mathcal{S}$ by letting $\mathcal{F} \mathcal{A M}\left(\left(B, t_{0}\right)\right)$ denote the set of pairs of the form

$$
\left((\mathcal{M}, B, \phi),\left\{\left(U_{j}, z_{j}\right) ; j \in \mathbb{N}\right\}\right)
$$

where $(\mathcal{M}, B, \phi)$ is a family such that the fiber of $\phi$ over $t_{0}$ (which is a compact complex manifold) occurs as a deformation of $M$ and $\left\{\left(U_{j}, z_{j}\right) ; j \in \mathbb{N}\right\}$ 
is a system of local coordinates for $\mathcal{M}$ which induces local complex coordinate systems for each of the fibers of the family. For simplicity of notation, we will denote such a pair just by $(\mathcal{M}, B, \phi)$, but agree to remember that a system of local coordinates (as above) for $\mathcal{M}$ has been fixed and should be used throughout in constructions involving this pair.

Let $P=\{p\}$ denote the degenerate one-point manifold consisting of a single point $p$. It is then clear that $\mathcal{F} \mathcal{A M}((P, p))=S$.

Let $(\mathcal{M}, B, \phi)$ be an element of $\mathcal{F} \mathcal{A M}\left(\left(B, t_{0}\right)\right)$. Now if $f: B^{\prime} \longrightarrow$ $B$ is a map of connected manifolds, we see that for the induced family $\left(f^{*} \mathcal{M}, B^{\prime}, f^{*} \phi\right)$ on $B^{\prime}$ (see (2.2.3.3) and (2.4.3.3)), if $t_{0}^{\prime} \in B^{\prime}$ is mapped onto $t_{0}$ by $f$, then $\left(f^{*} \phi\right)^{-1}\left(t_{0}^{\prime}\right)$ is biholomorphic to $\phi^{-1}\left(t_{0}\right)$ and hence this induced family is an element of the set $\mathcal{F} \mathcal{A M}\left(\left(B^{\prime}, t_{0}^{\prime}\right)\right)$. Thus we get a map of sets

$$
f^{*}: \mathcal{F} \mathcal{A M}\left(\left(B, t_{0}\right)\right) \longrightarrow \mathcal{F} \mathcal{A M}\left(\left(B^{\prime}, t_{0}^{\prime}\right)\right),(\mathcal{M}, B, \phi) \mapsto\left(f^{*} \mathcal{M}, B^{\prime}, f^{*} \phi\right)
$$

We also get a natural map of sets

$$
\operatorname{Hom}_{\mathcal{C}}\left(\left(B^{\prime}, t_{0}^{\prime}\right),\left(B, t_{0}\right)\right) \longrightarrow \operatorname{Hom}_{\mathcal{S}}\left(\mathcal{F} \mathcal{A} \mathcal{M}\left(\left(B, t_{0}\right)\right), \mathcal{F} \mathcal{A} \mathcal{M}\left(\left(B^{\prime}, t_{0}^{\prime}\right)\right)\right)
$$

given by $f \mapsto f^{*}$, where $\operatorname{Hom}_{\mathcal{A}}(X, Y)$ denotes the set of morphisms from an object $X$ to another object $Y$ of a category $\mathcal{A}$. We thus see that $\mathcal{F} \mathcal{A M}$ : $C \longrightarrow \mathcal{S}$ is a contravariant functor and is in fact an example of a contravariant functor of families arising naturally from a moduli problem viz. the problem of moduli of $M$.

Next, for an object $\left(B, t_{0}\right)$ in $\mathcal{C}$, we define the equivalence relation $\sim_{\left(B, t_{0}\right)}$ on the set $\mathcal{F} \mathcal{A M}\left(\left(B, t_{0}\right)\right)$ as follows. Define $(\mathcal{M}, B, \phi) \sim_{\left(B, t_{0}\right)}(\mathcal{N}, B, \psi)$ if and only if these two families are equivalent (in the sense of (2.2.3.1) or (2.4.3.1) as the case may be).

When $\left(B, t_{0}\right)=(P, p)$, we see that the equivalence relation $\sim_{\left(B, t_{0}\right)}$ on the set $\mathcal{F} \mathcal{A M}\left(\left(B, t_{0}\right)\right)=S$ reduces to the equivalence relation $\sim_{S}$. Finally, let $f:\left(B^{\prime}, t_{0}^{\prime}\right) \longrightarrow\left(B, t_{0}\right)$ be a morphism in $\mathcal{C}$. Then we get the induced map (via the contravariant functor $\mathcal{F} \mathcal{A M}$ ) given by

$$
\mathcal{F} \mathcal{A M}(f)=f^{*}: \mathcal{F} \mathcal{A M}\left(\left(B, t_{0}\right)\right) \longrightarrow \mathcal{F} \mathcal{A} \mathcal{M}\left(\left(B^{\prime}, t_{0}^{\prime}\right)\right)
$$

Now let $(\mathcal{M}, B, \phi)$ and $(\mathcal{N}, B, \psi)$ be elements of $\mathcal{F} \mathcal{A M}\left(\left(B, t_{0}\right)\right)$ which are equivalent (under the equivalence relation $\sim\left(B, t_{0}\right)$ ).

Then the induced families $\left(f^{*} \mathcal{M}, B^{\prime}, f^{*} \phi\right)$ and $\left(f^{*} \mathcal{N}, B^{\prime}, f^{*} \psi\right)$ that are elements of $\mathcal{F} \mathcal{A M}\left(\left(B^{\prime}, t_{0}^{\prime}\right)\right)$ are also equivalent under the equivalence relation $\sim\left(B^{\prime}, t_{0}^{\prime}\right)$.

Thus the equivalence on families defined above is compatible with the pullback operation on families. 


\subsubsection{The Functor of Equivalence Classes of Families}

We continue with the notations of the previous subsection. Define the contravariant functor $\mathcal{F}: \mathcal{C} \longrightarrow \mathcal{S}$, called the functor of equivalence classes of families, by

$$
\mathcal{F}\left(\left(B, t_{0}\right)\right)=\mathcal{F} \mathcal{A} \mathcal{M}\left(\left(B, t_{0}\right)\right) / \sim_{\left(B, t_{0}\right)}
$$

where $\mathcal{F} \mathcal{A M}\left(\left(B, t_{0}\right)\right) / \sim_{\left(B, t_{0}\right)}$ denotes the set of $\sim_{\left(B, t_{0}\right)}$-equivalence classes of families in $\mathcal{F} \mathcal{A M}\left(\left(B, t_{0}\right)\right)$. Notice that $\mathcal{F}((P, p))=S / \sim_{S}$. Further for a morphism $f:\left(B^{\prime}, t_{0}^{\prime}\right) \longrightarrow\left(B, t_{0}\right)$ in $\mathcal{C}$, we define

$$
\mathcal{F}(f): \mathcal{F}\left(\left(B, t_{0}\right)\right) \longrightarrow \mathcal{F}\left(\left(B^{\prime}, t_{0}^{\prime}\right)\right)
$$

by sending the equivalence class of $(\mathcal{M}, B, \phi)$ to the equivalence class of $\left(f^{*} \mathcal{M}, B^{\prime}, f^{*} \phi\right)$; this map is well-defined because the operation of pullback of families is compatible with the equivalence on families as indicated in the previous subsection.

We may now reformulate the problem of global moduli of $M$ to be a study of the properties of the functor $\mathcal{F}$, and the problem of local moduli of $M$ to be a study of the properties of the functor $\mathcal{F}_{l o c}$ obtained similarly by restricting attention only to sufficiently small deformations of $M$.

In the next section, we construct two families of complex tori which exhibit many special properties.

\subsection{Two Motivating Examples of Families of Complex Tori}

We construct two complex analytic families of complex tori which will serve as motivating examples for studying various phenomena naturally occurring in Deformation Theory and Moduli Theory. Both of the following constructions are due to Kodaira-Spencer ([6], Chap. VI, Sec. 14( $\gamma)$ ).

\subsubsection{The Complex Analytic Family $\mathcal{B}$ of Complex Tori}

Let $S$ be the set of complex matrices of order $n$ whose imaginary part has positive determinant. We write an element $s \in S$ as $s=\left(s_{\beta}^{\alpha}\right)$ where $\alpha$ denotes the row index and $\beta$ denotes the column index. Then $S$ is identified with an open submanifold, of the complex manifold $\mathbb{C}^{n^{2}}$, of matrices of order $n$ with $s_{\beta}^{\alpha}$ denoting the $(n(\alpha-1)+\beta)$-th coordinate on $\mathbb{C}^{n^{2}}$. For each matrix $s \in S$ we define a period matrix with $n$ rows and $2 n$ columns denoted $\omega(s)=\left(\omega_{j}^{\alpha}(s)\right)$ by setting $\omega_{j}^{\alpha}(s)=\delta_{j}^{\alpha}$ for $j=1, \ldots, n$ and setting 
$\omega_{j}^{\alpha}(s)=s_{\beta}^{\alpha}$ for $j=n+\beta, \beta=1, \ldots, n$. Define the following set of mappings of $\mathbb{C}^{n} \times S$ onto itself:

$$
\widehat{G}=\left\{g_{j}:(z, s) \mapsto\left(z+\omega_{j}(s), s\right) ; j=1, \ldots, 2 n\right\},
$$

where $\omega_{j}(s)$ is the vector in $\mathbb{C}^{n}$ with components corresponding to the entries in the $j$-th column of $\omega(s)$. Each $g_{j}$ is a holomorphic automorphism of $\mathbb{C}^{n} \times S$. Let $G$ denote the additive abelian subgroup of automorphisms of $\mathbb{C}^{n} \times S$ generated by $\widehat{G}$. $G$ acts properly discontinuously without fixed points and hence the quotient $\mathcal{B}:=\left(\mathbb{C}^{n} \times S\right) / G$ is a complex manifold by theorem (1.5.2.1.2); further, the canonical projection onto the second factor from $\mathbb{C}^{n} \times S$ induces a holomorphic map $\phi: \mathcal{B} \longrightarrow S$ whose fiber over each point $s \in S$ is a complex torus of dimension $n$ with period matrix $\omega(s)$ (see $(1.5 .2 .2 .1))$.

It is easily checked that the Jacobian of the map $\phi$ is of maximal rank at each point of $\mathcal{B}$. Thus $(\mathcal{B}, S, \phi)$ is a complex analytic family of complex $n$-dimensional tori.

For the case $n=1$, this family reduces to the complex analytic family of elliptic curves parametrized by the upper half-plane in $\mathbb{C}$ that we constructed in (2.2.2.2) (cf. (2.4.2)).

Next we construct, using a similar procedure as above but this time allowing arbitrary period matrices, a complex analytic family of complex $n$-dimensional tori $(\mathcal{C}, M, \psi)$ which has a larger parameter space than the above family $(\mathcal{B}, S, \phi)$. We shall establish certain remarkable properties of the family $(\mathcal{B}, S, \phi)$ in (3.4.4).

\subsubsection{The Complex Analytic Family $\mathcal{C}$ of Complex Tori}

Let $L$ be the space of all complex $n \times 2 n$ matrices $\omega=\left(\omega_{j}^{\alpha}\right)$ with the following additional condition:

$$
(\sqrt{-1})^{n} \operatorname{det}\left[\begin{array}{l}
\omega \\
\bar{\omega}
\end{array}\right]>0 .
$$

(When we write $\omega=\left(\omega_{j}^{\alpha}\right), j$ denotes the column index and $\alpha$ denotes the row index.) $L$ is identified in an obvious manner with an open submanifold of $\mathbb{C}^{2 n^{2}}$. Define the following set of mappings of $\mathbb{C}^{n} \times L$ onto itself:

$$
\widehat{G}=\left\{g_{j}:(z, \omega) \mapsto\left(z+\omega_{j}, \omega\right) ; j=1, \ldots, 2 n\right\},
$$

where $\omega_{j}$ is the vector in $\mathbb{C}^{n}$ with components corresponding to the entries in the $j$-th column of $\omega$. Each $g_{j}$ is a holomorphic automorphism of $\mathbb{C}^{n} \times$ $L$. Let $G$ be the additive abelian subgroup of automorphisms of $\mathbb{C}^{n} \times L$ generated by $\widehat{G}$. $G$ acts properly discontinuously and hence the quotient 
$\mathcal{L}:=\left(\mathbb{C}^{n} \times L\right) / G$ is a complex manifold; further, the canonical projection onto the second factor from $\mathbb{C}^{n} \times L$ induces a holomorphic map $\psi: \mathcal{L} \longrightarrow L$ whose fiber over each point $\omega \in L$ is a complex torus of dimension $n$ with period matrix $\omega$.

It is easily checked that the Jacobian of the map $\psi$ is of maximal rank at each point of $\mathcal{L}$. Thus $(\mathcal{L}, L, \psi)$ is a complex analytic family of complex $n$-dimensional tori. But the parameter space $L$ contains redundant parameters which we next eliminate.

There is a canonical left-action of $\mathbf{G L}(n, \mathbb{C})$ on $\mathbb{C}^{n}$ and $L$. The quotient $M:=L / \mathbf{G L}(n, \mathbb{C})$ is a complex manifold. We define the action of $\tau \in$ $\mathbf{G L}(n, \mathbb{C})$ on $\mathbb{C}^{n} \times L$ by the holomorphic automorphism (which we again denote by $\tau)$ given by $\tau:(z, \omega) \mapsto(\tau(z), \tau \omega)$. This map $\tau$ commutes with each element $g_{j} \in G$ and hence induces a holomorphic automorphism of $\mathcal{L}$, which we again denote by the same symbol $\tau$. Therefore $\mathbf{G L}(n, \mathbb{C})$ becomes a group of holomorphic automorphisms of $\mathcal{L}$. The quotient $\mathcal{C}:=$ $\mathcal{L} / \mathbf{G L}(n, \mathbb{C})$ is a complex manifold with a canonical holomorphic map into $M$, induced by $\psi$, which we again denote by $\psi$. The fibers of this map are again complex $n$-dimensional tori. Thus $(\mathcal{C}, M, \psi)$ is a complex analytic family of complex $n$-dimensional tori.

We can regard the above complex analytic family as being obtained in the following way also. In fact the main difference is that while in the above construction we have first taken the quotient of $\mathbb{C}^{n} \times L$ by $G$ to obtain $\mathcal{L}$ and then taken the quotient of $\mathcal{L}$ by $\mathbf{G L}(n, \mathbb{C})$ to obtain $\mathcal{C}$, in the construction below we will do just the reverse - the main point here is that the actions of $G$ and $\mathbf{G L}(n, \mathbb{C})$ commute at the appropriate instances.

Now $\mathbb{C}^{n} \times L$ can be thought of as the total space of the trivial holomorphic vector bundle of rank $n$ on $L$. Then the action of $\mathbf{G L}(n, \mathbb{C})$ on this total space as well as the base space $L$ of this vector bundle is compatible with respect to the bundle projection (i.e., the canonical projection from $\mathbb{C}^{n} \times L$ onto the second factor) — we express this fact by saying that $\mathbf{G L}(n, \mathbb{C})$ acts on the trivial bundle over $L$ of rank $n$. Let $\mathcal{E}=\left(\mathbb{C}^{n} \times L\right) / \mathbf{G L}(n, \mathbb{C})$ so that $\mathcal{E}$ is the total space of a holomorphic vector bundle over $M$ with a canonical bundle projection map onto $M$.

Now $G$ acts on $\mathbb{C}^{n} \times L$ and hence (remembering that the actions of $G$ and $\mathbf{G L}(n, \mathbb{C})$ on $\mathbb{C}^{n} \times L$ commute) $G$ acts also on $\mathcal{E}$ discontinuously. Further, under this action of $G$ on $\mathcal{E}$, the fibers of $\mathcal{E}$ over $M$ are left invariant. Thus the quotient $\mathcal{E} / G$ is a complex manifold and comes with a canonical holomorphic map into $M$; this quotient is none other than the complex manifold $\mathcal{C}$ we obtained above and the canonical map is simply the map $\psi$ so that we have again obtained the complex analytic family $(\mathcal{C}, M, \psi)$.

It is routine to verify that $M$ is covered by $\left(\begin{array}{c}2 n \\ n\end{array}\right)$ local complex coordinate neighborhoods $S_{j_{1}, j_{2} \ldots j_{n}}$ where $1 \leq j_{1}<\cdots<j_{n} \leq 2 n$, each of which is 
biholomorphic to the parameter space $S$ of the complex analytic family $(\mathcal{B}, S, \phi)$ of (2.6.1) above. Further, the restrictions (2.4.3.4) of the family $(\mathcal{C}, M, \psi)$ to these local complex coordinate neighborhoods are all equivalent to the complex analytic family $(\mathcal{B}, S, \phi)$. In fact, if we define the subsets

$$
L_{j_{1} j_{2} \ldots j_{n}}=\left\{\omega \in L ; \operatorname{det}\left(\omega_{j_{\beta}}^{\alpha}\right) \neq 0\right\}
$$

of $L$, then it is clear that these are open subsets, that they are invariant under the action of $\mathbf{G L}(n, \mathbb{C})$ and that they cover $L$ because of the condition given by equation $(2.1)$ above. Then the quotient by $\mathbf{G L}(n, \mathbb{C})$ of each subset $L_{j_{1} j_{2} \ldots j_{n}}$ is precisely the local complex coordinate neighborhood of $M$ denoted above by $S_{j_{1} j_{2} \ldots j_{n}}$. Thus we may identify $S$ with $S_{12 \ldots n}$ and $(\mathcal{B}, S, \phi)$ with the restriction of $(\mathcal{C}, M, \psi)$ to $S_{12 \ldots n}$.

Now let $\gamma \in \mathbf{S L}(2 n, \mathbb{R})$. Then the linear transformation of $L$ given by multiplication by $\gamma$ on the right induces an analytic automorphism of $M=L / \mathbf{G L}(n, \mathbb{C})$ denoted by $t \mapsto t \gamma$. In the case when both $t$ and $t \gamma$ belong to $S \subset M$, we have the explicit expression for $t \gamma$ as

$$
t \gamma=\left(\gamma_{11}+t \gamma_{21}\right)^{-1}\left(\gamma_{12}+t \gamma_{22}\right), \text { where } \gamma=\left[\begin{array}{ll}
\gamma_{11} & \gamma_{12} \\
\gamma_{21} & \gamma_{22}
\end{array}\right]
$$

Clearly $\mathbf{S L}(2 n, \mathbb{R})$ acts transitively on $M$ i.e., there is only one orbit for this action. Thus for any fixed $t \in M$, the quotient of $\mathbf{S L}(2 n, \mathbb{R})$ by the stabilizer subgroup of $t$ is isomorphic to $M$ itself.

Next let us consider the discrete subgroup of $\mathbf{S L}(2 n, \mathbb{R})$ given by $\mathbf{S L}(2 n, \mathbb{Z})$. It is then easily verified that two fibers $\psi^{-1}(t)$ and $\psi^{-1}(u)$ are conformally equivalent tori if and only if both $t$ and $u$ belong to the same orbit of $\mathbf{S L}(2 n, \mathbb{Z})$. Thus $M$ also contains redundant parameters and to eliminate these we must therefore go modulo the group $\mathbf{S L}(2 n, \mathbb{Z})$. Then it happens that the cases corresponding to $n=1$ and $n>1$ are quite different as explained below.

- When $n=1$ it is easy to check that the action of $\mathbf{S L}(2, \mathbb{Z})$ on $M$ is discontinuous and the quotient space $M / \mathbf{S L}(2, \mathbb{Z})$ is biholomorphic to $\mathbb{C}$ (cf. chap. 1 , sec. 1.5 where we worked with the upper half-plane $U$ and $\mathbf{P S L}(2, \mathbb{Z})$ respectively instead of $M$ and $\mathbf{S L}(2, \mathbb{Z}))$. However, the situation for the case $n>1$ is comparatively bad.

- When $n>1$ the action of $\mathbf{S L}(2 n, \mathbb{Z})$ on $M$ is not discontinuous (this result is due to Siegel [8]). In fact, it can be shown that every open non-empty subset $V$ of $M$ contains a point $t$ whose $\mathbf{S L}(2 n, \mathbb{Z})$-orbit intersects $V$ in an infinite set of points! (this is due to KodairaSpencer [6], Chap. VI, Sec. 14). Therefore we also deduce that the quotient topological space $M / \mathbf{S L}(2 n, \mathbb{Z})$ is not even Hausdorff! 


\section{Hence the natural construction of the moduli space of complex $n$-dimensional tori fails for $n>1$ !}

It can be further shown that for any nonempty open subset $V$ of $M$, the restriction of the family $(\mathcal{C}, M, \psi)$ to $V$ can never consist of conformally distinct tori, even if we want to consider the restricted family only as a differentiable family.

In fact, given a nonempty open subset $V$ of $M$ and a differentiable family $(\mathcal{V}, V, \xi)$ of complex $n$-dimensional tori each of whose fibers $\xi^{-1}(v), v \in V$, occurs (up to isomorphism) as a fiber of the restriction of $(\mathcal{C}, M, \psi)$ to $V$, the following can be proved: if the fibers of $\mathcal{V}$ are all conformally distinct, then $\mathcal{V}$ cannot contain a representative (up to isomorphism) of each of the fibers of $\mathcal{C}$ restricted to $V$ i.e., there exists $v_{0} \in V$ such that $\psi^{-1}\left(v_{0}\right)$ is not biholomorphic to any fiber $\xi^{-1}(v)$ of $\mathcal{V}$; in other words, if $\mathcal{V}$ does contain among its fibers a representative of every one of the fibers of $\mathcal{C}$ restricted to $V$, then there exist distinct point $v_{1}, v_{2}$ of $V$ such that $\xi^{-1}\left(v_{1}\right)$ and $\xi^{-1}\left(v_{2}\right)$ are biholomorphic. For proofs of these, refer to Chap. VI, Sec. $14(\gamma)$ of the paper of Kodaira-Spencer [6].

We shall establish certain remarkable "local moduli-theoretic" properties of the family $(\mathcal{C}, M, \psi)$ in (3.4.4).

In the next section we show how certain complex-analytic deformations of a complex-analytic object that admits an algebraic structure, may fail to admit any algebraic structures.

\subsection{Algebraizability and Analytic Deformations}

The study of the problem of moduli of a compact connected complex manifold when the manifold admits also an algebraic structure (for e.g., the problem of moduli of a compact Riemann surface), becomes very interesting and greatly unifies the vast subjects of Analysis, Differential Geometry, Differential Topology, Algebraic Topology, Number Theory and Algebraic Geometry; for such a study involves the correlation of various results and ideas from each of these branches of Mathematics with those of the others. The necessity for such a correlation arises from the fact that the object (whose moduli we want to study) has both an analytic as well as an algebraic structure and these structures are not entirely independent of each other - for example, many properties of a scheme over $\mathbb{C}$ (an algebraic structure described in (A.4) and (A.5) of the Appendix) imply the existence of corresponding properties for its associated complex analytic space and conversely (see theorem (A.10.4.1)).

It is therefore often helpful to know various conditions under which a 
given complex analytic space admits an algebraic structure. Some of the results concerning algebraizability of complex analytic spaces relevant to our needs are briefly presented in section A.10 of the Appendix.

It is not an uncommon phenomenon that a (global or local) holomorphic deformation of a complex algebraizable manifold (i.e., a complex manifold which is the complex analytic space associated to a scheme of finite type over $\mathbb{C}$ - see (A.10) of the Appendix) may fail to be algebraic; thus one must make suitable restrictions on the nature of the deformations considered if one wants to get only those analytic deformations which are also algebraic. We will discuss below an example of this phenomenon.

To begin with, we first recall a fundamental theorem that gives necessary and sufficient conditions under which a complex $n$-dimensional torus is algebraizable.

\subsubsection{Algebraizability of Complex Tori}

Let us recall the example of (1.5.2.2.1), where we defined the $n$-dimensional complex torus $T_{\omega}^{n}=\mathbb{C}^{n} / G$ with

$$
G=\left\{\sum_{j=1}^{2 n}\left(m_{j} w_{j}\right) ; m_{j} \in \mathbb{Z}\right\}
$$

and with $\langle\omega\rangle=\left\langle w_{1}, \ldots, w_{2 n}\right\rangle$ a set of $2 n$ vectors that are linearly independent over $\mathbb{R}$. When $n=1$ we know that each such torus $T_{\tau}^{1}$ is algebraic and is in fact an elliptic algebraic curve (1.5.2.6).

However, for $n>1$ almost all tori $T_{\omega}^{n}$ do not admit any algebraic structure!

To describe the ones that do, we first recall from (1.5.2.2.1) that the period matrix associated to $T_{\omega}^{n}$ is the transpose $\Omega^{T}$ of the matrix $\Omega=\left(w_{i}^{j}\right)$ with $2 n$ rows and $n$ columns, where we have written $w_{i}=\left(w_{i}^{1}, \ldots, w_{i}^{n}\right) \in \mathbb{C}^{n}$ for each $i=1, \ldots, 2 n$. We then have the following.

2.7.1.1 Theorem (Algebraizability of Complex Tori). A complex $n$-dimensional torus $T_{\omega}^{n}$ is algebraizable if and only if its period matrix $\Omega^{T}$ is a Riemann Matrix i.e., there exists a skew-symmetric (or alternating) real invertible matrix $J$ of order $2 n$ with integer entries such that the matrix of order $n$ given by $\Omega^{T} \cdot J^{-1} \cdot \Omega$ is the zero matrix and the determinant of the matrix of order $n$ given by $\sqrt{-1}\left(\bar{\Omega}^{T} \cdot J^{-1} \cdot \Omega\right.$ ) (which is a Hermitian matrix) is positive. Further, if $n \geq 2$, then almost all tori $T_{\omega}^{n}$ are $\underline{\text { not algebraizable. }}$

Notice that the hypothesis that the period matrix $\Omega^{T}$ is a Riemann matrix is equivalent to the following: there exists a Hermitian form $H$ on 
$\mathbb{C}^{n}$ such that (1) the real skew-symmetric (or alternating) form $E:=\operatorname{Im}(H)$ is integral on $G \times G \subset \mathbb{C}^{n} \times \mathbb{C}^{n}$ and (2) $H$ is positive definite.

Under these conditions it is possible to construct an ample line bundle $L$ on $T_{\omega}^{n}$ i.e., such that the space of holomorphic sections of the $m$ th tensor power of $L$ for $m \geq 3$ gives an embedding of $T_{\omega}^{n}$ as a closed complex submanifold in a suitable complex projective space $P_{\mathbb{C}}^{k}$. Then by the GAGA correspondence (or Chow's theorem) stated in (A.10.5) of the Appendix, it follows that $T_{\omega}^{n}$ is algebraizable. For further details, refer to Chap.1 of Mumford's book on Abelian Varieties [9], especially the Theorem of Lefschetz in section 3 there.

2.7.1.2 Remark. For $n=1$ the above theorem says that complex onedimensional tori $T^{1}=\mathbb{C} / G$ are always algebraizable. For if $G$ is the lattice in $\mathbb{C}$ generated by $1, \tau$ with $\operatorname{Im}(\tau)>0$, then the mapping $H: \mathbb{C} \times \mathbb{C} \longrightarrow \mathbb{C}$ given by $H(z, w)=(z \bar{w}) /(\operatorname{Im}(\tau))$ is a positive definite Hermitian form such that $\operatorname{Im}(H)$ is integral on $G \times G$. It is also easy to check in this case that the period matrix of $T^{1}$ is trivially a Riemann matrix.

However, as stated in the theorem above, almost all tori $T^{n}$ for $n \geq 2$ are not algebraic. It turns out that on most general tori $T^{n}, n \geq 2$ there exist no nonconstant meromorphic functions and hence they can't be algebraic. In fact a complex $n$-dimensional torus that admits $n$ algebraically independent meromorphic functions has to be algebraizable. For a proof of this, refer to section 3 of Chap. 1 of Mumford's book cited above.

\subsubsection{Non-algebraic Deformations of Complex Algebraic Tori}

Recall the examples of (2.6.1) and (2.6.2) of complex analytic families of complex tori denoted respectively by $(\mathcal{B}, S, \phi)$ and $(\mathcal{C}, M, \psi)$. Let us assume that $n>1$ and choose a point of the parameter space over which the fiber as complex torus is algebraic (i.e., its period matrix is a Riemann matrix as explained in the previous subsection). We can explicitly show that in any neighborhood of this chosen point, there exists a point over which the fiber as complex torus is not algebraizable i.e., there exists a point over which the fiber as complex torus has period matrix which is not a Riemann matrix. In fact, the locus of points that correspond to algebraic tori has dimension $\leq n(n+1) / 2<n^{2}$ (see (3.4.4.2)).

Hence there exist arbitrarily small holomorphic deformations of any $n$-dimensional algebraic complex torus that are not algebraic for each $n>1$.

In the next section, we show by suitable examples how the variation of complex structure of $M_{t}=\phi^{-1}(t)$ for a complex analytic family $(\mathcal{M}, B, \phi)$ 
may be continuous (as $t$ varies in $B$ ) or the variation may exhibit sudden changes called "jump phenomena".

\subsection{Discontinuous and Continuous Variation of Complex Structure}

\subsubsection{Continuous Variation of Complex Structure}

The example of (2.4.2) of the complex analytic family $(\mathcal{M}, U, \phi)$ of complex 1-dimensional tori parametrized by the upper half-plane $U$ is a situation in which the complex structure of $M_{t}=\phi^{-1}(t)$ varies continuously as $t$ varies in $U$. This can be seen using the elliptic modular function $J(t)$ of (1.5.2.6.3) since the conformal structure on the torus defined by $t \in U$ is completely characterized by the complex number $J(t)$.

\subsubsection{Discontinuous Variation of Complex Structure: Jump Phenomena}

A complex $n$-dimensional torus $T^{n}=\mathbb{C}^{n} / L$ where $L$ is a lattice in $\mathbb{C}^{n}$ is an obvious generalization of an elliptic curve $T^{1}=\mathbb{C} / G$ where $G$ is a lattice in $\mathbb{C}$ (cf. (1.5.2.2.1)). Though all $T^{1}$ are algebraic, we know from (2.7.1) that for $n>1$ most $T^{n}$ are not. However, their underlying differentiable (hence also topological) structures are the same by (2.3.1.1).

Another way of defining an elliptic curve $T^{1}=\mathbb{C} / G$ is by considering it to be the quotient $\mathbb{C}^{*} / G^{*}$ where $\mathbb{C}^{*}=\mathbb{C}-\{0\}$ and

$$
G^{*}=\left\{g_{m}^{*}: z \mapsto \alpha^{m} z ; \alpha=e^{2 \pi i \tau}, m \in \mathbb{Z}\right\}
$$

and where $G$ is the lattice $L=L(\tau)=\{n+m \tau ; n, m \in \mathbb{Z}\}$. Then $G^{*}$ acts properly discontinuously and without fixed points on $\mathbb{C}^{*}$ with fundamental domain

$$
F^{*}=\{z \in \mathbb{C}-\{0\} ;|\alpha| \leq|z| \leq 1\}
$$

which is a closed annulus so that $\mathbb{C}^{*} / G^{*}$ is a complex 1-dimensional torus by theorem (1.5.2.1.2). Note that the condition $\operatorname{Im}(\tau)>0$ implies that the modulus of the complex number $\alpha$ is less than 1 .

This definition of an elliptic curve as $T^{1}=\mathbb{C}^{*} / G^{*}$ can also be generalized to higher dimensions. However, the objects so obtained are very different from higher-dimensional complex tori: they are called Hopf manifolds and are not algebraizable. In fact, in contrast to the case of complex tori, even the underlying topological structure of a Hopf manifold is quite different from that of an algebraizable complex manifold. 
We shall construct a complex analytic family of Hopf surfaces (i.e., Hopf manifolds of complex dimension two) which exhibits a sudden change in complex structure at a point of the parameter space.

So let $G$ be the infinite cyclic group generated by the automorphism

$$
\left(z_{1}, \ldots, z_{n}\right) \mapsto\left(\alpha_{1} z_{1}, \ldots, \alpha_{n} z_{n}\right)
$$

of $\mathbb{C}^{n}-\{0\}$ where each $\alpha_{i}$ is a fixed complex constant of modulus greater than 1 . $G$ acts properly discontinuously and without fixed points on $\mathbb{C}^{n}-$ $\{0\}$ so that the quotient $M:=\left(\mathbb{C}^{n}-\{0\}\right) / G$ is a compact complex manifold (by (1.5.2.1.2)) called a Hopf manifold of dimension $n$.

$M$ as a real differentiable manifold can be shown to be isomorphic to the product $S^{1} \times S^{2 n-1}$ (where $S^{k}$ denotes the $k$-sphere in $\mathbb{R}^{k+1}$ ). Notice that for $n=1, M$ is just an elliptic curve of the form $\mathbb{C}^{*} / G^{*}$ as described above. For $n \geq 2$, on the one hand, the rank of the finitely generated abelian homology group $H_{1}\left(M_{t o p}, \mathbb{Z}\right)$-where $M_{t o p}$ is the topological space underlying $M$ - called the first Betti number of $M$ turns out to be equal to 1 . On the other hand, from the Hodge Theory of harmonic differential forms it is known that the first Betti number of an algebraic manifold is even. Therefore even the underlying topological structure of the Hopf manifold $M$ is different from that of an algebraic manifold.

It can be shown that if $\alpha_{1}, \ldots, \alpha_{n}$ are independent in the sense that $\alpha_{1}^{k_{1}} \ldots \alpha_{n}^{k_{n}} \neq 1$ for any $n$-tuple $\left(k_{1}, \ldots, k_{n}\right) \neq(0, \ldots, 0)$ of integers, then $M$ has no nontrivial meromorphic functions. This again implies that $M$ cannot be algebraizable, for any algebraic manifold abounds in such functions.

Now we construct a complex analytic family of Hopf surfaces i.e., a family of holomorphically varying complex structures on the differentiable manifold $S^{1} \times S^{3}$. So fix $\alpha, t \in \mathbb{C}$ such that $0<|\alpha|<1$ and define the map

$$
g_{t}:\left(z_{1}, z_{2}\right) \mapsto\left(\alpha z_{1}+t z_{2}, \alpha z_{2}\right) .
$$

This is a holomorphic automorphism of $\mathbb{C}^{2}-\{0\}$ and generates an infinite cyclic group $G_{t}$ that acts properly discontinuously and without fixed points on $\mathbb{C}^{2}-\{0\}$. So the quotient $M_{t}:=\left(\mathbb{C}^{2}-\{0\}\right) / G_{t}$ is a complex manifold (by (1.5.2.1.2)) called a Hopf surface since it is a Hopf manifold of dimension two. We claim that $\left\{M_{t} ; t \in \mathbb{C}\right\}$ is a complex analytic family. To see this, first define the mapping

$$
g:\left(z_{1}, z_{2}, t\right) \mapsto\left(\alpha z_{1}+t z_{2}, \alpha z_{2}, t\right)
$$

which is an automorphism of $\left(\mathbb{C}^{2}-\{0\}\right) \times \mathbb{C}$ and generates an infinite cyclic group $G$ that acts properly discontinuously and without fixed points on $\left(\mathbb{C}^{2}-\{0\}\right) \times \mathbb{C}$. Thus the quotient $\mathcal{M}:=\left(\left(\mathbb{C}^{2}-\{0\}\right) \times \mathbb{C}\right) / G$ is a complex manifold by (1.5.2.1.2). The second canonical projection from $\left(\mathbb{C}^{2}-\{0\}\right) \times \mathbb{C}$ 
commutes with $g$ and therefore induces a holomorphic map $\phi: \mathcal{M} \longrightarrow \mathbb{C}$. The rank of the Jacobian matrix of $\phi$ is 1 everywhere and hence $(\mathcal{M}, \mathbb{C}, \phi)$ is a complex analytic family of compact complex manifolds (Hopf surfaces) with fiber over $t \in \mathbb{C}$ given by $\phi^{-1}(t)=\left(\mathbb{C}^{2}-\{0\}\right) / G_{t}=M_{t}$.

Now let $t \in U:=\mathbb{C}-\{0\}$. The matrix of $g_{t}$ is given by $\left[\begin{array}{ll}\alpha & t \\ 0 & \alpha\end{array}\right]$. This upon conjugation by the matrix $\left[\begin{array}{ll}1 & 0 \\ 0 & t\end{array}\right]$ gives the matrix $\left[\begin{array}{ll}\alpha & 1 \\ 0 & \alpha\end{array}\right]$, which is just the matrix of $g_{1}$.

The above observation shows that the restriction of $(\mathcal{M}, \mathbb{C}, \phi)$ to $U$ (for a suitable choice of coordinates on $\mathcal{M}$ ) is equivalent to the trivial family over $U$ with fiber type $M_{1}$. Thus we see that $M_{t}$ has the same complex structure as $M_{1}$ for $t \neq 0$.

By nontrivial calculations involving holomorphic vector fields, Kodaira and Spencer have shown that while each $M_{t}, t \neq 0$ has only two linearly independent holomorphic vector fields, $M_{0}$ has four linearly independent holomorphic vector fields (see [6], chap. VI, sec. 15). Hence $M_{0}$ has to have a different complex structure from $M_{t}$ for each $t \neq 0$.

Thus the complex structure of $(\mathcal{M}, \mathbb{C}, \phi)=\left\{M_{t} ; t \in \mathbb{C}\right\}$ jumps at $t=0$.

This type of phenomenon is christened a jump phenomenon.

The occurrence of jump phenomena is one of the main reasons for the nonexistence of certain types of "global moduli spaces" called coarse moduli spaces and fine moduli spaces which we shall introduce in section 4.5 of chapter 4 .

In the next chapter we study the notion of an "arbitrarily small" deformation of complex structure called an infinitesimal deformation. 


\section{Theory of Local Moduli: Infinitesimal Deformations}

\subsection{Overview of this Chapter}

This chapter explains Kodaira-Spencer Theory, which is the study of arbitrarily small deformations of the complex structure on a given compact complex manifold.

Section 3.2 is devoted to the definition and properties of the various avatars of the Kodaira-Spencer maps for a given family of compact complex manifolds.

The notion of an infinitesimal deformation of the complex structure of a compact complex manifold, as an element of the first cohomology group of this manifold with values in its sheaf of germs of holomorphic vector fields, is introduced in subsection 3.2.1 for a differentiable family, and in subsection 3.2.8 for a complex analytic family.

Each infinitesimal deformation is associated to a tangent direction at the point, of the base space, over which the corresponding fiber of the family is the fixed compact complex manifold whose local deformations of complex structure we want to study. Therefore, we get a map of vector spaces, from the tangent space at that point of the base, to the first cohomology group of the compact complex manifold with values in its sheaf of holomorphic vector fields. This map is called the infinitesimal Kodaira-Spencer map and is introduced in subsection 3.2.2 for a differentiable family and in subsection 3.2.8 for a complex analytic family.

Since there is a differentiable family underlying every complex analytic family, it is natural to ask if there is any relationship between the infinitesimal Kodaira-Spencer map for a complex analytic family to that of the 
underlying differentiable family at a given point of the base manifold. That these maps may be considered to be one and the same in a suitable sense, is explained in subsection 3.2.9.

If a family of compact complex manifolds is locally trivial, then the infinitesimal Kodaira-Spencer maps at each point of the base manifold vanish. However, the converse is not generally true because of existence of families that exhibit jump phenomena. A converse does exist, if we impose the additional hypothesis of regularity which stipulates that the dimensions, of the first cohomology groups of the fibers with values in their corresponding sheaves of holomorphic vector fields, remain constant. This is explained in subsection 3.2.7 for a differentiable family and in subsection 3.2.8 for a complex analytic family.

One naturally asks if there exists a concept which would truly characterize the dependence of the variation of the complex structures, on the fibers of a family, on the varying point of the base space. Though the concept of an infinitesimal Kodaira-Spencer map does not satisfy this requirement unless the family is regular, there is a global version of this map which fits the bill. This is called the global Kodaira-Spencer map for the given family and is explained in subsection 3.2.6 for a differentiable family and in subsection 3.2.10 for a complex analytic family. The development of this concept requires the notions of fundamental sequences of vector bundles and sheaves for the given family, and these notions are described in subsections 3.2.3 and 3.2.5 for a differentiable family, and in subsection 3.2.10 for a complex analytic family. As a by-product of the discussion in subsection 3.2.3, one is able to formulate the notion of a differentiable family in terms of the sophisticated language of differentiable fiber bundles, and this formulation is explained in the subsequent subsection viz. 3.2.4.

As may be expected, the global Kodaira-Spencer map for a complex analytic family is related to that of the underlying differentiable family. This relationship is explained in subsection 3.2.11.

The infinitesimal deformation of a compact complex manifold along a prescribed tangent direction at the point of the base (over which the fiber of the family is the compact complex manifold in consideration) is obtained by differentiating the transition functions with respect to the tangent direction, and then expressing the derivatives in an intrinsic form by letting them to be coefficients of vector fields - this is how one ends up with a first cohomology class of the compact complex manifold with values in its sheaf of holomorphic vector fields. Therefore one may expect that the infinitesimal deformation should possess some well-known properties of the derivative. One such property is expressed by the chain rule, for computing infinitesimal deformations under a change of parameter, which is explained in subsection 3.3.1. 
Using the chain rule, one is able to give an example of a family for which all the infinitesimal deformation maps vanish, but yet the family is not locally trivial. This example is explained in subsection 3.3.2.

Not every element of the first cohomology group of a compact complex manifold with values in its sheaf of holomorphic vector fields corresponds to an infinitesimal deformation of its complex structure. In fact, there may exist certain elements - obstruction classes - which will not correspond to a smooth deformation of the complex structure. The notion of primary obstruction to infinitesimal deformation is formulated in subsection 3.4.1, and higher order obstructions are formulated in subsection 3.4.5. It is seen that, if the second cohomology group of the compact complex manifold with values in its sheaf of holomorphic vector fields vanishes, then all these obstructions vanish! Therefore, such a situation should be a good one, and this is justified by the Theorem of Existence, which says that in such a situation, all first cohomology classes are associated to infinitesimal deformations and this association is actually a correspondence. This theorem is indicated in subsection 3.4.6.

A family is said to be complete at a point of the base space, if it locally induces every other family of deformations of the compact complex manifold which is the fiber over this point. Completeness is ensured by the surjectivity of the infinitesimal Kodaira-Spencer map at that point, and this is the content of the Theorem of Completeness described in subsection 3.4.2.

Given a compact complex manifold, one may ask if there is a minimal set of independent parameters which are sufficient to parametrize every one of its infinitesimal deformations. If such a set exists, then its cardinality may be called the number of moduli of the compact complex manifold. The conditions under which the number of moduli is defined and exists are explained in subsections 3.4.3 and 3.4.6.

In subsection 3.4.4, we show the existence of the conditions required to define the number of moduli of any complex $n$-dimensional torus, and prove that this number is $n^{2}$.

The concept of a deformation of the complex structure on a compact complex manifold has an analogue for complex fiber bundles; the notion of a deformation of the complex structure on a complex fiber bundle, the notions of global and infinitesimal Kodaira-Spencer maps for a family of complex fiber bundles and various results analogous to those in the context of deformations of compact complex manifolds are indicated in section 3.5.

Every cohomology class in the first cohomology group, of a compact complex manifold with values in its sheaf of holomorphic vector fields, does correspond to an infinitesimal deformation of the complex structure on the compact complex manifold, provided one allows complex-analytic deformations which may in general not be smooth. This is the content of 
Kuranishi's Theorem, and is explained in section 3.6, necessitating the formulation using complex-analytic spaces which may not be manifolds i.e., which may in general have singularities. The notion of a local moduli space is also explained in this section.

Examples of local moduli spaces are given in section 3.7. Riemann's celebrated formula for the number of moduli of a compact Riemann surface is justified in subsection 3.7.1.

The existence of a local moduli space for a simple vector bundle on a compact Riemann surface is explained in section 3.8, and the dimension of this local moduli space is computed using the Riemann-Roch formula.

Deformation theory may be formulated in the category of schemes, and the corresponding definitions are explained in section 3.9.

The notion of a global moduli space, in contrast to that of a local moduli space, is explained in the next chapter. That these notions are related, and that one can obtain global moduli spaces by glueing local moduli spaces is also illustrated in the next chapter.

\subsection{Infinitesimal Deformations and Deformation Maps of Kodaira-Spencer}

\subsubsection{Infinitesimal Deformations for Differentiable Families}

Let $(\mathcal{M}, B, \phi)$ be a differentiable family of compact complex manifolds. Recall theorem (2.3.2.4): for $t_{0} \in B$ let $M_{t_{0}}=\phi^{-1}\left(t_{0}\right)$ be the compact connected complex manifold obtained by glueing the polydiscs $V_{1}, \ldots, V_{l}$ by identifying $z_{k} \in V_{k}$ and $z_{j}=f_{j k}\left(z_{k}\right) \in V_{j}$ via the transition functions $\left\{f_{j k}\right\}$ arising out of a given finite system of local complex coordinates $\left\{\left(U_{i}, z_{i}\right) ; i=\right.$ $1, \ldots, l\}$ i.e., $z_{j}\left(U_{j}\right)=V_{j}$ and $f_{j k}=z_{j} \circ z_{k}^{-1}$; then there exists a domain $I \subset B$ containing $t_{0}$ such that for each $t \in I, M_{t}=\phi^{-1}(t)$ is a compact complex manifold obtained by glueing the same polydiscs $V_{1}, \ldots, V_{l}$ but by different transition functions

$$
f_{j k}^{\alpha}\left(z_{k}, t\right)=f_{j k}^{\alpha}\left(z_{k}, t_{1}, \ldots, t_{m}\right)
$$

with the initial conditions $f_{j k}^{\alpha}\left(z_{k}, t_{0}\right)=f_{j k}^{\alpha}\left(z_{k}\right)$ where $t=\left(t_{1}, \ldots, t_{m}\right)$ are local $C^{\infty}$ coordinates on $I$ and the functions $f_{j k}\left(z_{k}, t\right)$ are $C^{\infty}$ functions of $t$.

In the following discussion, we will use the notations of the above theorem (and also of its proof). Recall that any fiber $M_{t}=\phi^{-1}(t)$ for $t \in I$ is called a local $C^{\infty}$-deformation of complex structure of $M_{t_{0}}$ (see (2.3.2.5)). In the following discussion we work with the restriction of $(\mathcal{M}, B, \phi)$ to $I$ i.e., with $\left(\mathcal{M}_{I}, I, \phi\right)$ and without loss of generality we assume that $I$ is a domain in $\mathbb{R}^{m}$. 
Recall that $\left\{\left(U_{j}^{\prime}, x_{j}\right) ; j=1, \ldots, l\right\}$ cover $\phi^{-1}(I)$ and that $x_{j}\left(U_{j}^{\prime}\right)=$ $V_{j} \times I_{j}$. Further, for each $t \in I$, let $U_{j, t}:=U_{j}^{\prime} \cap M_{t}$. Then, for each $t \in I$, we get the following finite system of local complex coordinates on $M_{t}$ :

$$
\begin{gathered}
\left\{\left(U_{j}^{\prime}, z_{j}\right) ; j=1, \ldots, l\right\}, \\
z_{j}^{\alpha}=x_{j}^{2 \alpha-1}+(\sqrt{-1}) x_{j}^{2 \alpha}(1 \leq \alpha \leq n), \\
x_{j}\left(U_{j, t}\right)=V_{j} \times\{t\} \cong V_{j} .
\end{gathered}
$$

We write $U_{j, t_{0}}$ as $U_{j}$. Notice first of all that on each nonempty intersection $U_{i}^{\prime} \cap U_{j}^{\prime} \cap U_{k}^{\prime}$ we have the following patching conditions for the transition functions:

$$
f_{i k}\left(z_{k}, t\right)=f_{i j}\left(f_{j k}\left(z_{k}, t\right), t\right) .
$$

Recall that $m$ is the dimension of $B$ as real differentiable manifold and $t=\left(t_{1}, \ldots, t_{m}\right)$ are local $C^{\infty}$ coordinates on $I$ induced from the standard ones on $\mathbb{R}^{m}$. Choose a $C^{\infty}$ tangent vector to $B$ at $t \in I$ of the form

$$
\frac{\partial}{\partial t}=\sum_{\lambda=1}^{m} c_{\lambda} \frac{\partial}{\partial t_{\lambda}}, c_{\lambda} \in \mathbb{R}
$$

which is an element of the $C^{\infty}$ tangent space to $B$ at $t \in I$, denoted $T_{t} B$. Introduce the following holomorphic vector fields given by

$$
\theta_{j k}\left(z_{j}, t\right)=\sum_{\alpha=1}^{n} \frac{\partial f_{j k}^{\alpha}\left(z_{k}, t\right)}{\partial t} \frac{\partial}{\partial z_{j}^{\alpha}}, \quad z_{k}=f_{k j}\left(z_{j}, t\right)
$$

on $U_{j, t} \cap U_{k, t}(\neq \emptyset) \subset M_{t}$.

It is important to bear in mind that in the above, the functions $f_{j k}^{\alpha}\left(z_{k}, t\right)$ of the variables $z_{k}^{1}, \ldots, z_{k}^{n}, t$ are first to be differentiated with respect to $t$ and then the substitutions $z_{k}^{\alpha}=f_{k j}^{\alpha}\left(z_{j}, t\right)$ are to be made.

We will denote $\theta_{j k}\left(z_{j}, t\right)$ by $\theta_{j k}(t)$ for simplicity. Differentiation of the condition (3.1) combined with the definition of $\theta_{j k}(t)$ from formula (3.2) gives the following identities on $U_{i, t} \cap U_{j, t} \cap U_{k, t}(\neq \emptyset) \subset M_{t}$ :

$$
\theta_{i k}(t)=\theta_{i j}(t)+\theta_{j k}(t), \theta_{k j}(t)=-\theta_{j k}(t)
$$

Let $\Theta_{t}$ denote the sheaf of germs of holomorphic vector fields over $M_{t}$ i.e., the sheaf associated to the holomorphic tangent bundle of $M_{t}$. Then the above relations express the fact that the sections $\theta_{j k}(t) \in \Theta_{t}\left(U_{j, t} \cap U_{k, t}\right)$ define a 1-cocycle $\left\{\theta_{j k}(t)\right\}$ with values in the sheaf $\Theta_{t}$ relative to the open covering $\mathcal{U}_{t}:=\left\{U_{j, t}\right\}$ of $M_{t}$ (see (A.9)). This is written as $\left\{\theta_{j k}(t)\right\} \in Z^{1}\left(\mathcal{U}_{t}, \Theta_{t}\right)$. 
Since each $U_{j, t}$ is biholomorphic (via $x_{j}$ ) to the corresponding polydisc $V_{j} \times\{t\} \cong V_{j}$ as noted earlier, we see from (A.9) that this 1-cocyle defines an element

$$
\theta(t) \in \mathrm{H}^{1}\left(M_{t}, \Theta_{t}\right) \cong \check{\mathrm{H}}^{1}\left(M_{t}, \Theta_{t}\right):=\check{\mathrm{H}}^{1}\left(\mathcal{U}_{t}, \Theta_{t}\right)
$$

It can be verified that the definition of $\theta(t)$ is independent of the choice of local $C^{\infty}$ coordinates $\left\{\left(U_{j}^{\prime}, x_{j}\right)\right\}$.

Since $\theta(t)$ is obtained by differentiating the patching conditions (3.1) for transition functions that are responsible for changing the complex structure of $M_{t}$ with respect to $t$, it is intuitively correct to make the following definition.

3.2.1.1 Definition. The cohomology class $\theta(t)$ obtained above is called the infinitesimal deformation of $M_{t}$ along the direction prescribed by $\frac{\partial}{\partial t} \in T_{t} B$. $\theta(t)$ is also called the derivative of the complex structure of $M_{t}$ along the direction prescribed by $\frac{\partial}{\partial t} \in T_{t} B$. Thus we write $\theta(t)=\frac{\partial M_{t}}{\partial t}$.

3.2.1.2 Note. We have defined $\theta(t)$ only for $t \in I$. However, the theorem (2.3.2.4) guarantees that as $t_{0}$ varies in $B$, neighborhoods such as $I$ always exist and cover $B$ and hence $\theta(t)$ is defined uniquely for each $t \in B$. At this point we again state that the definition of $\theta(t)$ does not depend on the choice of local $C^{\infty}$ coordinates on $\mathcal{M}$.

\subsubsection{Infinitesimal Kodaira-Spencer Maps for Differentiable Families}

We continue using the notations of (3.2.1) and fix a differentiable family of compact complex manifolds $(\mathcal{M}, B, \phi)$ for the following discussion.

3.2.2.1 Definition. Define the mapping $\rho_{t}: T_{t} B \longrightarrow \mathrm{H}^{1}\left(M_{t}, \Theta_{t}\right)$ given by $\rho_{t}\left(\frac{\partial}{\partial t}\right)=\theta(t)=\frac{\partial M_{t}}{\partial t}$. This map is an $\mathbb{R}$-linear map and is called the infinitesimal deformation map or the infinitesimal Kodaira-Spencer map at $t \in B$ for the family $(\mathcal{M}, B, \phi)$.

If a function does not depend on a certain parameter then its partial derivative with respect to that parameter must be zero. Similarly, if the complex structure on $M_{t}$ does not depend on the parameter $t \in B$, we must have that the derivative of the complex structure, along any prescribed tangent direction at each $t \in B$, must be zero. This must all the more be expected in the case of a locally trivial family since in that case all the fibers are biholomorphic to each other. This expectation turns out to be true and is verified by straightforward computation. Hence we can state the following. 
3.2.2.2 Lemma. If $(\mathcal{M}, B, \phi)$ is a locally trivial differentiable family of compact complex manifolds, then at each point $t \in B$, the infinitesimal Kodaira-Spencer map $\rho_{t}$ is the zero map. In other words, locally trivial families do not admit any infinitesimal deformations.

Suppose we were to believe that $\rho_{t}$ truly characterizes the dependency of complex structure of $M_{t}$ on $t \in B$ locally, then it is natural to expect that the vanishing of $\rho_{t}$ at each point $t \in B$ must imply that the differentiable family is locally trivial. Unfortunately this is not true (because of jump phenomena (cf. (2.8.2) and (3.3.2)) unless we impose some additional conditions - see theorem (3.2.7.3). However, it turns out that there is a "global version" of the infinitesimal Kodaira-Spencer map which overcomes this difficulty. We describe this in (3.2.6). Before that, we define the notions of fundamental sequences for a differentiable family and also explain the reformulation of the notion of differentiable family in terms of differentiable fiber bundles in subsections (3.2.3) through (3.2.5) below.

\subsubsection{The Fundamental Sequence of Vector Bundles for a Differentiable Family}

Let $(\mathcal{M}, B, \phi)$ be a differentiable family of compact complex manifolds. We use the notations of theorem (2.3.2.4) and its proof. Consider the transition functions $\left\{f_{j k}\right\}$ that glue the domains $\left\{V_{j} \times I_{j}\right\}$ to give $\mathcal{M}$. For $p \in U_{i}^{\prime} \cap U_{k}^{\prime}(\neq \emptyset)$ we set as usual $z_{i}^{\alpha}(p)=f_{i k}^{\alpha}\left(z_{k}(p), t\right),(\alpha=1, \ldots, n)$. These are $C^{\infty}$ functions which are actually holomorphic in $z_{k}$. Let $g_{i k}(p)$ be the Jacobian matrix

$$
g_{i k}(p):=\left[\frac{\partial z_{i}^{\alpha}(p)}{\partial z_{k}^{\beta}(p)}\right] .
$$

Let $\mathbb{F}$ be the complex vector bundle over $\mathcal{M}$ determined by the system of transition functions $\left\{g_{i k}\right\}$ with respect to the open cover $\left\{U_{i}^{\prime}\right\}$ of $\mathcal{M}$. Next let $\mathbb{G}$ be the vector bundle over $\mathcal{M}$ with fiber $\mathbb{C}^{n} \oplus \mathbb{R}^{m}$ determined by the system of transition functions with respect to this cover given by matrices

$$
h_{i k}(p):=\left[\begin{array}{cc}
g_{i k}(p) & {\left[\partial z_{i}^{\alpha}(p) / \partial t_{k}^{\nu}\right]} \\
0 & {\left[\partial t_{i}^{\lambda} / \partial t_{k}^{\nu}\right]}
\end{array}\right],
$$

where $t_{i}=\left(t_{i}^{1}, \ldots, t_{i}^{m}\right)$ is a system of local $C^{\infty}$ coordinates for $B$ covering $\phi\left(U_{i}^{\prime}\right)$ for every $i$. Then clearly $\mathbb{F}$ is a sub-bundle of $\mathbb{G}$ and we have a short exact sequence of vector bundles on $\mathcal{M}$ given by

$$
0 \longrightarrow \mathbb{F} \longrightarrow \mathbb{G} \longrightarrow \mathbb{G} / \mathbb{F} \longrightarrow 0
$$


which is called the fundamental sequence of vector bundles for the differentiable family $(\mathcal{M}, B, \phi)$.

3.2.3.1 Remark. By thinking of any fixed fiber $M_{t}=\phi^{-1}(t)$ as a family over a base space consisting of a single point, we can similarly get a fundamental sequence of vector bundles for the fiber over $t \in B$.

\subsubsection{Reformulation of the Definition of Differentiable Family in Terms of Differentiable Fiber Bundles}

We now give another formulation of the notion of a differentiable family, as already promised in (2.2.1.4) and (2.3.1.2).

Let $\mathbb{F}_{+}$be the $C^{\infty}$ vector bundle over $\mathcal{M}$ of tangent vectors along fibers of $\phi$ and $\mathbb{G}_{+}$be the $C^{\infty}$ tangent bundle of $\mathcal{M}$. Recall that $m$ and $2 n$ are the dimensions respectively of the differentiable manifolds corresponding to $B$ and to any fiber of $\phi$. We have the short exact sequence of $C^{\infty}$ vector bundles over $\mathcal{M}$ given by

$$
0 \longrightarrow \mathbb{F}_{+} \longrightarrow \mathbb{G}_{+} \longrightarrow \mathbb{G}_{+} / \mathbb{F}_{+} \longrightarrow 0 \text {. }
$$

Let $\mathbf{G L}((2 n, m), \mathbb{R})$ denote the subgroup of elements of $\mathbf{G L}(2 n+m, \mathbb{R})^{1}$ which map the linear subspace $x_{2 n+1}=x_{2 n+2}=\cdots=x_{2 n+m}=0$ of $\mathbb{R}^{2 n+m}$ (with standard coordinates $x_{1}, \ldots, x_{2 n+m}$ ) onto itself. Then due to (2.3.1.1) the structure group of $\mathbb{G}_{+}$is $\mathbf{G L}((2 n, m), \mathbb{R})$ and that of $\mathbb{F}_{+}$is a subgroup of this group. Let $\mathbf{G L}(n, \mathbb{C} ; m, \mathbb{R})$ denote the group of matrices of the form

$$
\left[\begin{array}{ll}
A & B \\
0 & C
\end{array}\right],
$$

where $A \in \mathbf{G L}(n, \mathbb{C}), C \in \mathbf{G L}(m, \mathbb{R})$ and $B$ is an arbitrary $n \times m$ matrix with entries in $\mathbb{C}$. Notice that the matrices given by $h_{i k}(p)$ of (3.2.3) are of this form. Define the map $\mathbf{G L}(n, \mathbb{C} ; m, \mathbb{R}) \longrightarrow \mathbf{G L}((2 n, m), \mathbb{R})$ by

$$
\left[\begin{array}{cc}
A & B \\
0 & C
\end{array}\right] \mapsto\left[\begin{array}{ccc}
\operatorname{Re}(A) & -\operatorname{Im}(A) & \operatorname{Re}(B) \\
\operatorname{Im}(A) & \operatorname{Re}(A) & \operatorname{Im}(B) \\
0 & 0 & C
\end{array}\right] .
$$

Then this map embeds $\mathbf{G L}(n, \mathbb{C} ; m, \mathbb{R})$ as a real subgroup of $\mathbf{G L}((2 n, m), \mathbb{R})$. Put the matrices given by $h_{i k}(p)$ of (3.2.3) in the form

$$
\left[\begin{array}{ll}
A & B \\
0 & C
\end{array}\right],
$$

\footnotetext{
${ }^{1} \mathbf{G L}(k, \mathbb{R})$ (respectively $\mathbf{G L}(k, \mathbb{C})$ ) denotes the group of invertible real (respectively complex) matrices of order $k \in \mathbb{N}$.
} 
consider these as elements of $\mathbf{G L}((2 n, m), \mathbb{R})$ by the above embedding and take the $C^{\infty}$ vector bundle on $\mathcal{M}$ defined with these as transition functions. The resulting vector bundle turns out to be precisely $\mathbb{G}_{+}$. Now complexify the first $2 n$ components of vectors of $\mathbb{G}_{+}$to get a vector bundle $\mathcal{C} \mathbb{G}_{+}$. Let

$$
E=\left[\begin{array}{ccc}
1 & \sqrt{-1} & 0 \\
1 & -\sqrt{-1} & 0 \\
0 & 0 & 1
\end{array}\right]
$$

so that we have

$$
E \cdot\left[\begin{array}{ccc}
\operatorname{Re}(A) & -\operatorname{Im}(A) & \operatorname{Re}(B) \\
\operatorname{Im}(A) & \operatorname{Re}(A) & \operatorname{Im}(B) \\
0 & 0 & C
\end{array}\right] \cdot E^{-1}=\left[\begin{array}{ccc}
A & 0 & B \\
0 & \bar{A} & \bar{B} \\
0 & 0 & C
\end{array}\right]
$$

which shows that the vector bundle $\mathcal{C} \mathbb{G}_{+}$has $\mathbb{G}$ and its conjugate $\overline{\mathbb{G}}$ as sub-bundles. Next, if the vector bundle $\mathcal{C} \mathbb{F}_{+}$is the complexification of $\mathbb{F}_{+}$, then we have the splitting $\mathcal{C} \mathbb{F}_{+}=\mathbb{F}_{+} \oplus(\sqrt{-1}) \mathbb{F}_{+}=\mathbb{F} \oplus \overline{\mathbb{F}}$. Finally recall that $(\mathcal{M}, B, \phi)$ can be regarded as a differentiable fiber bundle because of (2.3.1.1). Hence we can make the following definition.

3.2.4.1 Definition. A differentiable family of complex manifolds is a differentiable fiber bundle $(\mathcal{M}, B, \phi)$ alongwith a differentiable reduction of the structure group $\mathbf{G L}((2 n, m), \mathbb{R})$ of the $C^{\infty}$ tangent bundle $\mathbb{G}_{+}$of $\mathcal{M}$ to $\mathbf{G L}(n, \mathbb{C} ; m, \mathbb{R})$ (regarded as real subgroup) which imparts to each fiber a complex analytic structure. Here $m$ and $2 n$ respectively denote the dimensions of $B$ and of any fiber of $\phi$ considered as real differentiable manifolds.

\subsubsection{The Fundamental Sequence of Sheaves for a Differentiable Family}

We continue with the notations of (3.2.3) above. Let $\Theta, \Psi$ respectively be the sheaves of germs of differentiable sections of $\mathbb{F}, \mathbb{G}$ whose restrictions to each fiber of $\phi$ are holomorphic. Then we get, from the fundamental sequence of vector bundles for the differentiable family $(\mathcal{M}, B, \phi)$ defined in (3.2.3) the following short exact sequence (cf. (A.8)) of sheaves on $\mathcal{M}$ :

$$
0 \longrightarrow \Theta \longrightarrow \Psi \stackrel{j}{\longrightarrow} \Psi / \Theta \longrightarrow 0 \text {. }
$$

Let $\Upsilon_{B}$ be the sheaf of germs of differentiable sections of the tangent bundle of $B$ and let $\widetilde{\Upsilon}=\phi^{-1}\left(\Upsilon_{B}\right)$ be the inverse image sheaf induced over $\mathcal{M}$ by 
$\phi($ see $(\mathrm{A} .1))$. Then there is a natural injection $\widetilde{\Upsilon} \hookrightarrow \Psi / \Theta$ and its image will be denoted by $\Upsilon$. Let $\Pi=j^{-1}(\Upsilon) \hookrightarrow \Psi$ so that we get a short exact sequence of sheaves (on $\mathcal{M}$ )

$$
0 \longrightarrow \Theta \longrightarrow \Pi \stackrel{j}{\longrightarrow} \Upsilon \longrightarrow 0
$$

called the fundamental sequence of sheaves for the differentiable family $(\mathcal{M}, B, \phi)$.

An alternative definition of the above fundamental sequence of sheaves is as follows. Let $\mathcal{O}_{\mathbb{R}}$ be the sheaf of germs of real-valued differentiable functions on $\mathcal{M}$ that are constant along fibers of $\phi . \Psi$ is a sheaf of germs of vector fields tangent to $\mathcal{M}$ and operates on $\mathcal{O}_{\mathbb{R}}$ by differentiation. Let $\Pi$ be the largest subsheaf of $\Psi$ for which $\mathcal{O}_{\mathbb{R}}$ is stable under the operation of $\Pi$ by differentiation. Then $\Theta$ is a subsheaf of $\Pi$ and $\Theta$ annihilates $\mathcal{O}_{\mathbb{R}}$. Then the quotient $\Pi / \Theta$ is nothing but $\Upsilon$ and thus we get the fundamental sequence of sheaves mentioned above.

3.2.5.1 Remark. By thinking of any fixed fiber $M_{t}=\phi^{-1}(t)$ as a family over a base space consisting of a single point, we can similarly get a fundamental sequence of sheaves for the fiber over $t \in B$.

\subsubsection{The Global Kodaira-Spencer Map for a Differentiable Family}

Let $(\mathcal{M}, B, \phi)$ be a differentiable family of complex manifolds. First of all, for a sheaf $\mathcal{F}$ of abelian groups on $\mathcal{M}$ and for each integer $q \geq 0$, consider the presheaf of abelian groups on $B$ defined by the association

$$
U \mapsto \mathrm{H}^{q}\left(\mathcal{M}_{U},\left.\mathcal{F}\right|_{\mathcal{M}_{U}}\right)
$$

for each open subset $U$ of $B$ where $\mathcal{M}_{U}:=\phi^{-1}(U)$ (see (A.1) and (A.8) for an explanation of the notations used here). Its sheafification (see (A.1)) will be denoted by $\mathcal{H}^{q}(\mathcal{F})$. Let

$$
0 \longrightarrow \Theta \longrightarrow \Pi \stackrel{j}{\longrightarrow} \Upsilon \longrightarrow 0
$$

be the fundamental sequence of sheaves for $(\mathcal{M}, B, \phi)$ introduced in (3.2.5) above. Let $U \subset B$ be any open subset. Then we have the following short exact sequence of sheaves

$$
\left.\left.\left.0 \longrightarrow \Theta\right|_{\mathcal{M}_{U}} \longrightarrow \Pi\right|_{\mathcal{M}_{U}} \stackrel{j}{\longrightarrow} \Upsilon\right|_{\mathcal{M}_{U}} \longrightarrow 0
$$


over $\mathcal{M}_{U}=\phi^{-1}(U)$ giving a corresponding long exact sequence in cohomology (see (A.8))

$$
\begin{aligned}
0 & \longrightarrow \mathrm{H}^{0}\left(\mathcal{M}_{U},\left.\Theta\right|_{\mathcal{M}_{U}}\right) \longrightarrow \mathrm{H}^{0}\left(\mathcal{M}_{U},\left.\Pi\right|_{\mathcal{M}_{U}}\right) \longrightarrow \\
& \longrightarrow \mathrm{H}^{0}\left(\mathcal{M}_{U},\left.\Upsilon\right|_{\mathcal{M}_{U}}\right) \stackrel{\delta_{U}}{\longrightarrow} \mathrm{H}^{1}\left(\mathcal{M}_{U},\left.\Theta\right|_{\mathcal{M}_{U}}\right) \longrightarrow \cdots
\end{aligned}
$$

where $\delta_{U}$ is the connecting homomorphism. Since

$$
\mathrm{H}^{0}\left(\mathcal{M}_{U},\left.\Upsilon\right|_{\mathcal{M}_{U}}\right) \cong \mathrm{H}^{0}\left(U,\left.\Upsilon_{B}\right|_{\mathcal{M}_{U}}\right)=\Upsilon_{B}(U)
$$

where $\Upsilon_{B}$ denotes the sheaf associated to the $C^{\infty}$ tangent bundle of $B$, we have (via $\delta_{U}$ ) the map

$$
\rho_{U}: \Upsilon_{B}(U) \longrightarrow \mathrm{H}^{1}\left(\mathcal{M}_{U},\left.\Theta\right|_{\mathcal{M}_{U}}\right)
$$

which for $U$ sufficiently small and containing a point $t \in B$ is called the local Kodaira-Spencer map at that point. This map is linear over the ring of differentiable functions over $U$.

We next vary $U$ in $B$ and sheafify the presheaf corresponding to each entry of the long exact sequence of cohomology groups mentioned above to obtain (because of the universal properties of the sheafifications - see (A.1)) a long exact sequence of sheaves of cohomology groups

$$
0 \longrightarrow \mathcal{H}^{0}(\Theta) \longrightarrow \mathcal{H}^{0}(\Pi) \longrightarrow \mathcal{H}^{0}(\Upsilon) \stackrel{\delta}{\longrightarrow} \mathcal{H}^{1}(\Theta) \longrightarrow \mathcal{H}^{1}(\Pi) \longrightarrow \cdots
$$

Since $\mathcal{H}^{0}(\Upsilon) \cong \Upsilon_{B}$, we get (via $\delta$ ) the homomorphism of sheaves

$$
\rho: \Upsilon_{B} \longrightarrow \mathcal{H}^{1}(\Theta)
$$

which is called the global Kodaira-Spencer map for the differentiable family $(\mathcal{M}, B, \phi)$. Note that $\rho$ is linear over the sheaf of germs of $C^{\infty}$ functions on $B$.

In the next subsection, we discuss the relationship between the local triviality of a family and the Kodaira-Spencer maps.

\subsubsection{Local Triviality and the Kodaira-Spencer Maps}

We state the following theorem which indicates the relationship between the global and infinitesimal versions of the Kodaira-Spencer maps for a differentiable family and which also justifies the statement that the global Kodaira-Spencer map truly characterizes the dependence on the parameter of the complex structures on the fibers of the family. For its proof, refer to the papers of Kodaira-Spencer [6], chap. II. 


\subsubsection{Theorem.}

1. Let $(\mathcal{M}, B, \phi)$ be a differentiable family of compact complex manifolds. Let $\rho_{t}: T_{t} B \longrightarrow H^{1}\left(M_{t}, \Theta_{t}\right)$ and $\rho: \Upsilon_{B} \longrightarrow \mathcal{H}^{1}(\Theta)$ denote respectively the infinitesimal Kodaira-Spencer map at $t \in B$ and the global Kodaira-Spencer map for this family. Then there exist canonical maps $r_{1, t}$ and $r_{2, t}$ such that the following diagram commutes

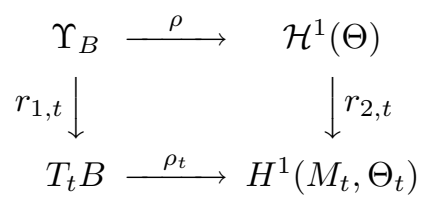

2. $(\mathcal{M}, B, \phi)$ is locally trivial if and only if $\rho$ vanishes.

3. $\rho=0$ implies that $\rho_{t}=0$ at each point $t \in B$ but the converse is not true.

In connection with assertion (3) of the above theorem, one can say more. Let $\rho$ vanish and fix a point $t \in B$. Choose a small open neighborhood $U$ of $t$ and first note that $\Upsilon_{B}(U)$ is a finite module over the ring of differentiable functions over $U$. So if $U$ is taken sufficiently small, the local KodairaSpencer map at $t \in B$ viz. $\rho_{U}$ itself must vanish.

On the other hand, if $\rho_{t}=0$ for each $t \in B$, we have already mentioned in (3.2.2) that the family need not be locally trivial. We will give an example in (3.3.2) of a differentiable family which is not locally trivial but for which all the infinitesimal Kodaira-Spencer maps vanish. Thus, by assertion (2) of the above theorem, the global Kodaira-Spencer map cannot vanish for this family. The family in this example is the family of Hopf surfaces constructed in (2.8.2). Recall from (2.8.2) that this family exhibits a jump in the variation of complex structure at a point of the parameter space. Calculations reveal that the dimension of the complex vector space, given by the first cohomology group of the fiber with values in its sheaf of germs of holomorphic vector fields, shoots up at the point $t$ at which the jump phenomenon occurs. It is therefore natural to expect that families $(\mathcal{M}, B, \phi)$ for which the dimension of $\mathrm{H}^{1}\left(M_{t}, \Theta_{t}\right)$ is a constant independent of $t \in B$ might behave better i.e., $\rho_{t}=0$ for each $t \in B$ may imply $\rho=0$ for such families. This motivates the following definition; the theorem following it justifies that the condition of the definition is indeed the right one.

3.2.7.2 Definition. The differentiable family $(\mathcal{M}, B, \phi)$ is said to be regular if and only if the dimension of the complex vector space $\mathrm{H}^{1}\left(M_{t}, \Theta_{t}\right)$ is the same for all points $t \in B$ i.e., it is an integer independent of $t \in B$. 
Here, as usual, $M_{t}=\phi^{-1}(t)$ for each $t \in B$, and $\Theta_{t}$ is the sheaf of germs of holomorphic vector fields on $M_{t}$.

3.2.7.3 Theorem. Let $(\mathcal{M}, B, \phi)$ be a regular differentiable family of compact complex manifolds. Then this family is locally trivial if and only if the infinitesimal Kodaira-Spencer map at each point of B vanishes.

The proof of the theorem is done by induction on the dimension of $B$ and involves the Theory of Differentiable Families of Strongly Elliptic Differential Operators. For further details, refer to chap. 7 of Kodaira's book [5].

3.2.7.4 Important Note. We again emphasize the indispensability of the assumption of regularity in the above theorem - see the counter-example in (3.3.2).

From the next theorem, a theorem of Fröhlicher-Nijenhuis [10] can be deduced. Their theorem asserts that any differentiable family of compact complex manifolds each biholomorphic to $n$-dimensional complex projective space $P_{\mathbb{C}}^{n}$ (for a fixed positive integer $n$ ) has to be locally trivial. Notice that the first cohomology group of $P_{\mathbb{C}}^{n}$ with values in the sheaf of germs of holomorphic vector fields is zero. It was this theorem that encouraged KodairaSpencer to make precise their philosophy that a deformation of a compact complex manifold is to be considered as the glueing of the same finite set of polydiscs but by different transition functions which depend (either differentiably or holomorphically) on a parameter, because this notion naturally gives rise to an element in $\mathrm{H}^{1}(M, \Theta)$ corresponding to each tangent direction at the point on the parameter space over which the fiber is $M$.

3.2.7.5 Theorem (Rigidity Principle). Let $(\mathcal{M}, B, \phi)$ be a differentiable family of compact complex manifolds. Let $t_{0} \in B$ and let the compact complex manifold $M_{t_{0}}:=\phi^{-1}\left(t_{0}\right)$ be such that $H^{1}\left(M_{t_{0}}, \Theta_{t_{0}}\right)=0$. Then there is a sufficiently small neighborhood $I \subset B$ of $t_{0}$ restricted to which this differentiable family becomes trivial.

Proof. The function $t \mapsto \operatorname{dim}_{\mathbb{C}}\left(\mathrm{H}^{1}\left(M_{t}, \Theta_{t}\right)\right)$ is an upper semicontinuous function. (This result depends on the theory of differentiable families of strongly elliptic differential operators. For a more general result of this type see (1) of theorem (3.2.11.2)). Hence there is a sufficiently small neighborhood $I$ of $t_{0}$ such that $\operatorname{dim}_{\mathbb{C}}\left(\mathrm{H}^{1}\left(M_{t}, \Theta_{t}\right)\right) \leq \operatorname{dim}_{\mathbb{C}}\left(\mathrm{H}^{1}\left(M_{t_{0}}, \Theta_{t_{0}}\right)\right)$ for each $t \in I$. This forces $\operatorname{dim}_{\mathbb{C}}\left(\mathrm{H}^{1}\left(M_{t}, \Theta_{t}\right)\right)=0$ for each $t \in I$. Thus the restriction $\left(\mathcal{M}_{I}, I, \phi\right)$ is a regular differentiable family. Further, for this family, at each point of the parameter space $I$, the infinitesimal Kodaira-Spencer map 
vanishes. Hence by the previous theorem this restricted family is locally trivial. Now we may choose $I$ sufficiently small so that the restricted family itself is trivial QED

Motivated by the above theorem, we state the following.

3.2.7.6 Definition (Rigid Manifold). A compact complex manifold $M$ is called rigid if for any differentiable family of compact complex manifolds $(\mathcal{M}, B, \phi)$ with $t_{0} \in B$ such that the compact complex manifold $M_{t_{0}}=\phi^{-1}\left(t_{0}\right)$ is biholomorphic to $M$, there is an open neighborhood of $t_{0}$ restricted to which this differentiable family becomes trivial.

Thus, if $M$ is rigid, it has no infinitesimal deformations and any small deformation of $M$ is still biholomorphic to $M$ itself.

The above theorem says that if $M$ is a compact complex manifold such that $\mathrm{H}^{1}(M, \Theta)=0$, then $M$ is rigid. Here, as usual, $\Theta$ denotes the sheaf of germs of holomorphic vector fields on $M$. Thus $P_{\mathbb{C}}^{n}$ is rigid for each positive integer $n$.

\subsubsection{Infinitesimal Deformations and Kodaira-Spencer Maps for Complex Analytic Families}

Let $(\mathcal{M}, B, \phi)=\left\{M_{t} ; t \in B\right\}$ be a complex analytic family of compact complex manifolds. Fix a point $t \in B$. By restricting this family (if necessary) to a local complex coordinate neighborhood of $t$, we may assume without loss of generality that $B$ is a domain in $\mathbb{C}^{m}$. Here $m$ denotes the dimension of $B$ as a complex manifold. Let $t=\left(t_{1}, \ldots, t_{m}\right)$ be the coordinates on $B$ induced from the standard coordinates on $\mathbb{C}^{m}$. Further, let $n$ denote the dimension of any fiber of $\phi$ as complex manifold. Thus the dimension of $\mathcal{M}$ as complex manifold is $(n+m)$ (recall that the Jacobian of $\phi$ is of maximal rank at each point of $\mathcal{M})$. Then from the remarks in (2.4.1.3) we can choose a system of local complex coordinates $\left\{\left(U_{j}, z_{j}\right) ; j \in \mathbb{N}\right\}$ on $\mathcal{M}$ such that the following hold:

1. the coordinate map $z_{j}: U_{j} \longrightarrow \mathbb{C}^{n+m}$ is given by

$$
p \mapsto\left(z_{j}^{1}(p), \ldots, z_{j}^{n}(p), t_{1}, \ldots, t_{m}\right)
$$

where $\phi(p)=\left(t_{1}, \ldots, t_{m}\right)$ i.e., locally $\phi$ looks like a projection:

$$
\left(z_{j}^{1}, \ldots, z_{j}^{n}, t_{1}, \ldots, t_{m}\right) \mapsto\left(t_{1}, \ldots, t_{m}\right) ;
$$

2. the open covering of $\mathcal{M}$ given by $\mathcal{U}=\left\{U_{j} ; j \in \mathbb{N}\right\}$ is locally finite; 
3. for each $t \in B$, the open covering induced by $\mathcal{U}$ on $M_{t}$ alongwith the maps

$$
\left\{p \mapsto\left(z_{j}^{1}(p), \ldots, z_{j}^{n}(p)\right), p \in U_{j} \cap M_{t} \neq \emptyset\right\}
$$

gives a system of local complex coordinates on $M_{t}$. The transition functions for $\mathcal{M}$ (with respect to the above coordinate system) are as usual given by $\left\{f_{j k}=z_{j} \circ z_{k}^{-1}\right\}$ and we write $z_{j}^{\alpha}=f_{j k}^{\alpha}\left(z_{k}, t\right), \alpha=$ $1, \ldots, n+m$.

Recall that a local holomorphic deformation of a compact complex manifold (corresponding to the fiber of $\phi$ over a point $t \in B$ ) is simply obtained by glueing the same polydiscs which were glued to get the given compact complex manifold, but via different transition functions which depend holomorphically on the parameter (cf. (2.3.2.4)). We want to measure how the glueing changes infinitesimally as $t$ varies in $B$. Since the nature of the glueing is determined by the transition functions $f_{j k}$, it is natural to differentiate these functions with respect to $t$ and view the resulting functions as a measure of the dependence on the parameter $t$ of the holomorphic structure on the fibers of $\phi$. We proceed below exactly as in the case of differentiable families (cf. (3.2.1)) to define infinitesimal deformations and infinitesimal Kodaira-Spencer maps for complex analytic families.

So let $T_{t} B$ denote the holomorphic tangent space of $B$ at $t$. Let a holomorphic tangent vector

$$
\frac{\partial}{\partial t}=\sum_{\lambda=1}^{m} c_{\lambda} \frac{\partial}{\partial t_{\lambda}}, c_{\lambda} \in \mathbb{C}
$$

be given. Define the following holomorphic vector field on $U_{j k}:=U_{j} \cap U_{k}$ :

$$
\theta_{j k}\left(z_{j}, t\right)=\sum_{\alpha=1}^{n} \frac{\partial f_{j k}^{\alpha}\left(z_{k}, t\right)}{\partial t} \frac{\partial}{\partial z_{j}^{\alpha}}, z_{k}=f_{k j}\left(z_{j}, t\right) .
$$

It is important to bear in mind that in the above, the functions $f_{j k}^{\alpha}\left(z_{k}, t\right)$ of the variables $z_{k}^{1}, \ldots, z_{k}^{n}, t$ are first to be differentiated with respect to $t$ and then the substitutions $z_{k}^{\alpha}=f_{k j}^{\alpha}\left(z_{j}, t\right)$ are to be made.

We will denote $\theta_{j k}\left(z_{j}, t\right)$ by $\theta_{j k}(t)$ for simplicity. Then, as in the case of differentiable families, simple differentiation yields the following identities on $U_{i} \cap U_{j} \cap U_{k}(\neq \emptyset)$ :

$$
\theta_{i k}(t)=\theta_{i j}(t)+\theta_{j k}(t), \theta_{k j}(t)=-\theta_{j k}(t) .
$$

The above relations express the fact that the sections $\theta_{j k}(t) \in \Theta_{t}\left(U_{j} \cap U_{k} \cap\right.$ $M_{t}$ ) (where $\Theta_{t}$ is the sheaf of germs of holomorphic vector fields over $M_{t}$ 
i.e., the sheaf associated to the holomorphic tangent bundle of $M_{t}$ ) give rise to a 1-cocycle $\left\{\theta_{j k}(t)\right\}$ with values in the sheaf $\Theta_{t}$ relative to the open covering $\mathcal{U}_{t}:=\left\{U_{j} \cap M_{t}\right\}$ of $M_{t}$. This is written as $\left\{\theta_{j k}(t)\right\} \in Z^{1}\left(\mathcal{U}_{t}, \Theta_{t}\right)$. We may choose $\mathcal{U}$ in such a way that each nonempty intersection $U_{j} \cap M_{t}$ is biholomorphic to a polydisc in $\mathbb{C}^{n}$. Therefore, this 1-cocycle defines a unique element of $\mathrm{H}^{1}\left(M_{t}, \Theta_{t}\right)$ (see (A.9)) which we denote by $\theta(t)$.

It can be verified that the definition of $\theta(t)$ is independent of the choice of local complex coordinates on $\mathcal{M}$.

Since $\theta(t)$ is obtained by differentiating the patching condition for transition functions which are responsible for changing the complex structure of $M_{t}$ with respect to $t$, it is intuitively correct to make the following definition.

3.2.8.1 Definition (Infinitesimal Deformations for Complex Analytic Families). The cohomology class $\theta(t)$ obtained above is called the infinitesimal deformation of $M_{t}$ along the direction prescribed by $\frac{\partial}{\partial t} \in T_{t} B$. $\theta(t)$ is also called the derivative of the complex structure of $M_{t}$ along the direction prescribed by $\frac{\partial}{\partial t} \in T_{t} B, t \in B$. Thus we write $\theta(t)=\frac{\partial M_{t}}{\partial t}$.

3.2.8.2 Definition (Infinitesimal Kodaira-Spencer Maps for Complex Analytic Families). We continue using the above notations with a complex analytic family of compact complex manifolds $(\mathcal{M}, B, \phi)$ fixed for the following discussion.

Define the mapping $\rho_{t}: T_{t} B \longrightarrow \mathrm{H}^{1}\left(M_{t}, \Theta_{t}\right)$ given by $\rho_{t}\left(\frac{\partial}{\partial t}\right)=\theta(t)=$ $\frac{\partial M_{t}}{\partial t}$. This map is a $\mathbb{C}$-linear map and is called the infinitesimal deformation map or the infinitesimal Kodaira-Spencer map at $t \in B$ for the complex analytic family $(\mathcal{M}, B, \phi)$.

As in the case of differentiable families, we obtain the following results (cf. (3.2.2.2), (3.2.7.3) and (3.2.7.5)).

\subsubsection{Theorem.}

1. If $(\mathcal{M}, B, \phi)$ is a locally trivial complex analytic family of compact complex manifolds, then at each point $t \in B$, the infinitesimal KodairaSpencer map $\rho_{t}$ is the zero map. In other words, locally trivial families do not admit any infinitesimal deformations.

2. Let $(\mathcal{M}, B, \phi)$ be a regular complex analytic family of compact complex manifolds. Then this family is locally trivial if and only if the infinitesimal Kodaira-Spencer map at each point of $B$ vanishes.

3. (Rigidity Principle). Let $(\mathcal{M}, B, \phi)$ be a complex analytic family of compact complex manifolds. Let $t_{0} \in B$ and let the compact complex manifold $M_{t_{0}}:=\phi^{-1}\left(t_{0}\right)$ be such that $H^{1}\left(M_{t_{0}}, \Theta_{t_{0}}\right)=0$. Then there is a sufficiently small neighborhood $I \subset B$ of $t_{0}$ restricted to which this complex analytic family becomes trivial. 
4. Let $(\mathcal{M}, B, \phi)=\left\{M_{t}:=\phi^{-1}(t) ; t \in B\right\}$ be a complex analytic family of compact complex manifolds such that $H^{1}\left(M_{t}, \Theta_{t}\right)=0$ for each $t \in B$. Then $(\mathcal{M}, B, \phi)$ is a complex analytic fiber bundle.

As an application of assertion (4) of the above theorem, we see that any complex analytic family of compact complex manifolds each biholomorphic to $P_{\mathbb{C}}^{n}$ has to be locally trivial and hence is a complex analytic fiber bundle (called a projective bundle) with structure group the projective group of linear transformations on $P_{\mathbb{C}}^{n}$.

3.2.8.4 Important Note. As for the indispensability of the assumption of regularity in (2) of the above theorem, see the counter-example in (3.3.2).

\subsubsection{The Relationships of the Infinitesimal Kodaira- Spencer Maps for a Complex Analytic Family to those of the Underlying Differentiable Family}

For the below discussion, we fix a complex analytic family of compact complex manifolds $(\mathcal{M}, B, \phi)$ and continue using the notations of the previous subsection.

For any complex manifold $N$, let $N_{\mathbb{R}}$ denote the underlying differentiable manifold. Then the dimension of $\mathcal{M}_{\mathbb{R}}$ is $(2 n+2 m)$ and that of any fiber of $\phi$ considered as a differentiable manifold, viz. $\left(M_{t}\right)_{\mathbb{R}}$, is $2 n$. Assume that $B_{\mathbb{R}} \subset\left(\mathbb{C}^{m}\right)_{\mathbb{R}}=\mathbb{R}^{2 m}$. Let $x=\left(x_{1}, \ldots, x_{2_{m}}\right)$ be the real $C^{\infty}$ coordinates on $B_{\mathbb{R}}$ induced by the standard coordinates on $\left(\mathbb{C}^{m}\right)_{\mathbb{R}}=\mathbb{R}^{2 m}$. In other words, $\mathbb{C}^{m}$ is identified with $\mathbb{R}^{2 m}$ (as differentiable manifolds) using standard coordinates where the complex coordinates $t=\left(t_{1}, \ldots, t_{m}\right)$ correspond to the real coordinates $x=\left(x_{1}, \ldots, x_{2_{m}}\right)$ via the relations $t_{j}=x_{2 j-1}+(\sqrt{-1}) x_{2 j},(1 \leq j \leq n)$. Then by direct calculation we have the relations

$$
\begin{aligned}
\frac{\partial}{\partial t_{j}} & =\frac{1}{2 \sqrt{-1}} \frac{\partial}{\partial x_{2 j}}+\frac{1}{2} \frac{\partial}{\partial x_{2 j-1}}, \\
\text { and } \frac{\partial}{\partial \overline{t_{j}}} & =\frac{-1}{2 \sqrt{-1}} \frac{\partial}{\partial x_{2 j}}+\frac{1}{2} \frac{\partial}{\partial x_{2 j-1}},
\end{aligned}
$$

from which follow the relations

$$
\begin{aligned}
\frac{\partial}{\partial t_{j}}+\frac{\partial}{\partial \overline{t_{j}}}=\frac{\partial}{\partial x_{2 j-1}} \\
\text { and } \frac{\partial}{\partial t_{j}}-\frac{\partial}{\partial \overline{t_{j}}}=-\sqrt{-1} \frac{\partial}{\partial x_{2 j}} .
\end{aligned}
$$


Now if we take $c_{\lambda}$ to be complex numbers, then the association

$$
\sum_{\lambda=1}^{2 m} c_{\lambda} \frac{\partial}{\partial x_{\lambda}} \leftrightarrow \sum_{\beta=1}^{m}\left(c_{2 \beta-1}+(\sqrt{-1}) c_{2 \beta}\right) \frac{\partial}{\partial t_{\beta}}+\sum_{\beta=1}^{m}\left(c_{2 \beta-1}-(\sqrt{-1}) c_{2 \beta}\right) \frac{\partial}{\partial \overline{t_{\beta}}}
$$

identifies as complex vector spaces the complexification of the $C^{\infty}$ tangent (real vector) space $T_{x}^{\mathbb{R}}\left(B_{\mathbb{R}}\right)$ to $B_{\mathbb{R}}$ at $x$, denoted by $T_{x}^{\mathbb{R}}\left(B_{\mathbb{R}}\right) \otimes_{\mathbb{R}} \mathbb{C}$, with the complex vector space $T_{t} B \oplus \overline{T_{t} B}$ where $\overline{T_{t} B}$ denotes the conjugate of the holomorphic tangent space $T_{t} B$ to $B$ at $t \in B$.

The underlying differentiable family of $(\mathcal{M}, B, \phi)$ is denoted by $\left(\mathcal{M}_{\mathbb{R}}, B_{\mathbb{R}}, \phi\right)$, where $\phi$ is thought of just as a $C^{\infty}$ map. To avoid confusion, we will modify our earlier notation for the infinitesimal Kodaira-Spencer map for the underlying differentiable family at $x \in B_{\mathbb{R}}$ to $\rho_{x}^{\mathbb{R}}$. The infinitesimal Kodaira-Spencer map at $x \in B_{\mathbb{R}}$ for the underlying differentiable family is given by

$$
\rho_{x}^{\mathbb{R}}: T_{x}^{\mathbb{R}}\left(B_{\mathbb{R}}\right) \longrightarrow \mathrm{H}^{1}\left(M_{t}, \Theta_{t}\right)
$$

where $\rho_{x}^{\mathbb{R}}\left(\frac{\partial}{\partial x}\right)=\theta(x)=\frac{\partial M_{t}}{\partial x}$ (here $t$ and $x$ represent the same point). More precisely, if we have the expression

$$
\frac{\partial}{\partial x}=\sum_{\lambda=1}^{2 m} c_{\lambda} \frac{\partial}{\partial x_{\lambda}},\left(c_{\lambda} \in \mathbb{R}\right)
$$

for the $C^{\infty}$ tangent vector $\frac{\partial}{\partial x}$, then $\theta(x)$ is the image of the 1-cocycle $\left\{\theta_{j k}(x)\right\}$ in $\mathrm{H}^{1}\left(M_{t}, \Theta_{t}\right)$ given by

$\theta_{j k}(x)=\sum_{\alpha=1}^{n} \frac{\partial f_{j k}^{\alpha}\left(z_{k}, x\right)}{\partial x} \frac{\partial}{\partial z_{j}^{\alpha}}=\sum_{\alpha=1}^{n}\left(\sum_{\lambda=1}^{2 m} c_{\lambda} \frac{\partial f_{j k}^{\alpha}\left(z_{k}, x\right)}{\partial x_{\lambda}}\right) \frac{\partial}{\partial z_{j}^{\alpha}}, z_{k}=f_{k j}\left(z_{j}, x\right)$.

Now replacing $x$ by $t$ and noting that the partial derivaties of $f_{j k}^{\alpha}$ with respect to $\overline{t_{\beta}}$ vanish because these functions are holomorphic in $t_{\beta}$, we get the following expression for $\theta_{j k}(x)=\theta_{j k}(t)$ :

$$
\theta_{j k}(t)=\sum_{\alpha=1}^{n}\left(\sum_{\beta=1}^{m}\left(c_{2 \beta-1}+(\sqrt{-1}) c_{2 \beta}\right) \frac{\partial f_{j k}^{\alpha}\left(z_{k}, t\right)}{\partial t_{\beta}}\right) \frac{\partial}{\partial z_{j}^{\alpha}}
$$

Therefore we see that

$$
\rho_{x}^{\mathbb{R}}\left(\sum_{\lambda=1}^{2 m} c_{\lambda} \frac{\partial}{\partial x_{\lambda}}\right)=\rho_{t}\left(\sum_{\beta=1}^{m}\left(c_{2 \beta-1}+(\sqrt{-1}) c_{2 \beta}\right) \frac{\partial}{\partial t_{\beta}}\right) .
$$


Now we can define a $\mathbb{C}$-linear map

$$
\widetilde{\rho_{x}^{\mathbb{R}}}: T_{x}^{\mathbb{R}}\left(B_{\mathbb{R}}\right) \otimes_{\mathbb{R}} \mathbb{C} \longrightarrow \mathrm{H}^{1}\left(M_{t}, \Theta_{t}\right)
$$

obviously by extending $\rho_{x}^{\mathbb{R}}$ so that we obtain

$$
\widetilde{\rho_{x}^{\mathbb{R}}}\left(\sum_{\lambda=1}^{2 m} c_{\lambda} \frac{\partial}{\partial x_{\lambda}}\right)=\rho_{t}\left(\sum_{\beta=1}^{m}\left(c_{2 \beta-1}+(\sqrt{-1}) c_{2 \beta}\right) \frac{\partial}{\partial t_{\beta}}\right)
$$

where we emphasize that the $c_{\lambda}$ are all complex numbers. Thus we get the following commutative diagram, where we have identified $T_{x}^{\mathbb{R}}\left(B_{\mathbb{R}}\right) \otimes_{\mathbb{R}} \mathbb{C}$ with $T_{t} B \oplus \overline{T_{t} B}$ :

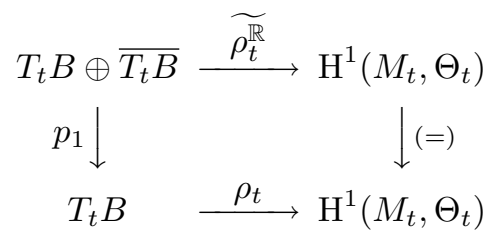

In the above diagram, $p_{1}$ denotes the projection onto the first direct summand. Next notice that the map $\widetilde{\rho_{t}^{\mathbb{R}}}$ vanishes identically on $\overline{T_{t} B}$.

Therefore we see that for a complex analytic family, the natural extension of the infinitesimal Kodaira-Spencer map of the underlying differentiable family can be identified with the infinitesimal Kodaira-Spencer map of the complex analytic family at each point of the parameter manifold.

It is obvious that if a complex analytic family $(\mathcal{M}, B, \phi)$ is locally trivial, then the underlying differentiable family is also locally trivial. However, using the above, we get the following converse.

3.2.9.1 Theorem. Let $(\mathcal{M}, B, \phi)$ be a complex analytic family of compact complex manifolds. Then this family is locally trivial if and only if the underlying differentiable family is locally trivial.

Proof. Assume that $(\mathcal{M}, B, \phi)$ is a complex analytic family such that its underlying differentiable family is locally trivial. This complex analytic family and its underlying differentiable family are both regular families because all fibers are biholomorphic to each other. By hypothesis the infinitesimal Kodaira-Spencer map for the underlying differentiable family vanishes at each point of the parameter space. Hence by the discussion above, so does the infinitesimal Kodaira-Spencer map for the complex analytic family at each point of $B$. Now apply (2) of theorem (3.2.8.3) of the previous subsection QED

We next state the following stronger version of the above theorem. 
3.2.9.2 Theorem. A complex analytic family is trivial if and only if its underlying differentiable family is trivial.

For a proof of this nontrivial theorem, see chap.4, sec. 2(c) of Kodaira's book [5]. The "only if" part of the statement of the above theorem is obvious. It is only the "if" part which is nontrivial to prove; notice that the previous theorem only guarantees that the complex analytic family is locally trivial, but its triviality is not at all obvious.

\subsubsection{The Global Kodaira-Spencer Map for a Complex Analytic Family}

We introduced in (3.2.3) the notion of a fundamental sequence of vector bundles for a differentiable family; then we introduced the notion of a fundamental sequence of sheaves for a differentiable family in (3.2.5) following which, in (3.2.6), we introduced the notion of a global Kodaira-Spencer map for a differentiable family. We discuss below the corresponding notions for a complex analytic family.

Let $(\mathcal{M}, B, \phi)$ be a complex analytic family of compact complex manifolds. For any holomorphic vector bundle $(\mathbb{B}, \mathcal{M}, \pi)$ over $\mathcal{M}$ with total space $\mathbb{B}$ and bundle projection $\pi$, let $\mathcal{D}(\mathbb{B})$ denote the sheaf over $\mathcal{M}$ of germs of holomorphic sections of this vector bundle (a section of $(\mathbb{B}, \mathcal{M}, \pi)$ over $\mathcal{M}$ is simply a set-theoretic map $\mathcal{M} \longrightarrow \mathbb{B}$ which when followed by $\pi$ gives the identity mapping of $\mathcal{M})$. Let $\mathcal{D}_{\mathbb{R}}(\mathbb{B})$ be the sheaf over $\mathcal{M}$ of germs of those $C^{\infty}$ sections of $(\mathbb{B}, \mathcal{M}, \pi)$ which are holomorphic along fibers of $\phi$.

Let $\mathbb{G}$ be the holomorphic tangent bundle of $\mathcal{M}$ and $\mathbb{F}$ the sub-bundle of $\mathbb{G}$ of tangent vectors along the fibers of $\phi$. Let $\Theta=\mathcal{D}(\mathbb{F})$ i.e., the sheaf of germs of holomorphic sections of $\mathbb{F}$ (or the sheaf of germs of holomorphic vector fields along the fibers of $\phi$ ).

Let $\Upsilon_{B}$ be the sheaf over $B$ of germs of holomorphic sections of the holomorphic tangent bundle of $B$ and $\Upsilon:=\phi^{-1}\left(\Upsilon_{B}\right)$ be its inverse image sheaf (see (A.1)) on $\mathcal{M}$. Finally let $\Pi$ denote the subsheaf of $\mathcal{D}(\mathbb{F})$ consisting of germs of holomorphic vector fields on $\mathcal{M}$ whose horizontal components are constant along fibers of $\phi$.

Then we have (analogous to the case of a differentiable family (3.2.3)) the short exact sequence of holomorphic vector bundles on $\mathcal{M}$ given by

$$
0 \longrightarrow \mathbb{F} \longrightarrow \mathbb{G} \longrightarrow \mathbb{G} / \mathbb{F} \longrightarrow 0
$$

called the fundamental sequence of vector bundles for the complex analytic family $(\mathcal{M}, B, \phi)$ and the short exact sequence of sheaves on $\mathcal{M}$ (cf. (3.2.5)) given by

$$
0 \longrightarrow \Theta \longrightarrow \Pi \stackrel{j}{\longrightarrow} \Upsilon \longrightarrow 0
$$


called the fundamental sequence of sheaves for the complex analytic family $(\mathcal{M}, B, \phi)$. Let $U \subset B$ be any open subset. Then we have the following short exact sequence

$$
\left.\left.\left.0 \longrightarrow \Theta\right|_{\mathcal{M}_{U}} \longrightarrow \Pi\right|_{\mathcal{M}_{U}} \stackrel{j}{\longrightarrow} \Upsilon\right|_{\mathcal{M}_{U}} \longrightarrow 0
$$

over $\mathcal{M}_{U}=\phi^{-1}(U)$ giving a corresponding long exact sequence in cohomology (see (A.8))

$$
\begin{aligned}
0 & \longrightarrow \mathrm{H}^{0}\left(\mathcal{M}_{U},\left.\Theta\right|_{\mathcal{M}_{U}}\right) \longrightarrow \mathrm{H}^{0}\left(\mathcal{M}_{U},\left.\Pi\right|_{\mathcal{M}_{U}}\right) \longrightarrow \\
& \longrightarrow \mathrm{H}^{0}\left(\mathcal{M}_{U},\left.\Upsilon\right|_{\mathcal{M}_{U}}\right) \stackrel{\delta_{U}}{\longrightarrow} \mathrm{H}^{1}\left(\mathcal{M}_{U},\left.\Theta\right|_{\mathcal{M}_{U}}\right) \longrightarrow \cdots
\end{aligned}
$$

where $\delta_{U}$ is the connecting homomorphism. Since $\mathrm{H}^{0}\left(\mathcal{M}_{U},\left.\Upsilon\right|_{\mathcal{M}_{U}}\right) \cong$ $\mathrm{H}^{0}\left(U, \Upsilon_{B}\right)=\Upsilon_{B}(U)$, we have (via $\left.\delta_{U}\right)$ the map

$$
\rho_{U}: \Upsilon_{B}(U) \longrightarrow \mathrm{H}^{1}\left(\mathcal{M}_{U},\left.\Theta\right|_{\mathcal{M}_{U}}\right)
$$

which for $U$ sufficiently small and containing a point $t \in B$ is called the local Kodaira-Spencer map at that point. This map is linear over the ring of holomorphic functions over $U$.

We next vary $U$ in $B$ and sheafify the presheaf corresponding to each entry of the long exact sequence of cohomology groups mentioned above to obtain (because of the universal properties of the sheafifications (see (A.1)) a long exact sequence of sheaves of cohomology groups

$$
0 \longrightarrow \mathcal{H}^{0}(\Theta) \longrightarrow \mathcal{H}^{0}(\Pi) \longrightarrow \mathcal{H}^{0}(\Upsilon) \stackrel{\delta}{\longrightarrow} \mathcal{H}^{1}(\Theta) \longrightarrow \mathcal{H}^{1}(\Pi) \longrightarrow \cdots
$$

Since $\mathcal{H}^{0}(\Upsilon) \cong \Upsilon_{B}$, we get (via $\delta$ ) the homomorphism of sheaves

$$
\rho: \Upsilon_{B} \longrightarrow \mathcal{H}^{1}(\Theta)
$$

which is called the global Kodaira-Spencer map for the complex analytic family $(\mathcal{M}, B, \phi)$. Note that $\rho$ is linear over the sheaf of germs of holomorphic functions on $B$.

We then have the following analogue of theorem (3.2.7.1).

\subsubsection{Theorem.}

1. Let $(\mathcal{M}, B, \phi)$ be a complex analytic family of compact complex manifolds. Let $\rho_{t}: T_{t} B \longrightarrow H^{1}\left(M_{t}, \Theta_{t}\right)$ and $\rho: \Upsilon_{B} \longrightarrow \mathcal{H}^{1}(\Theta)$ denote respectively the infinitesimal Kodaira-Spencer map at $t \in B$ and the global Kodaira-Spencer map for this family. Then there exist canonical maps $r_{1, t}$ and $r_{2, t}$ such that the following diagram commutes

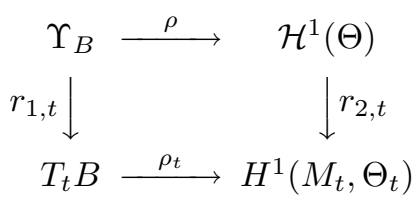


2. $(\mathcal{M}, B, \phi)$ is locally trivial if and only if $\rho$ vanishes.

3. $\rho=0$ implies that $\rho_{t}=0$ at each point $t \in B$ but the converse is not true (cf. assertion (2) of theorem (3.2.8.3)).

3.2.10.2 Remark. By thinking of any fixed fiber $M_{t}=\phi^{-1}(t)$ as a family over a base space consisting of a single point, we can similarly get fundamental sequences of vector bundles and sheaves for the fiber over $t \in B$.

In (3.2.9) we indicated the relationship of the infinitesimal KodairaSpencer map at a point for a complex analytic family to the infinitesimal Kodaira-Spencer map at the same point for the underlying differentiable family. In the same way, we will indicate below the relationship of the global Kodaira-Spencer map for a complex analytic family to the global Kodaira-Spencer map for the underlying differentiable family.

\subsubsection{The Relationship of the Global Kodaira-Spencer Map for a Complex Analytic Family to that of the Underlying Differentiable Family}

Let $(\mathcal{M}, B, \phi)$ be a complex analytic family of compact complex manifolds. Let $(\mathbb{B}, \mathcal{M}, \pi)$ be a holomorphic vector bundle on $\mathcal{M}$. We want to understand the relationship of $\mathrm{H}^{1}\left(\mathcal{M}_{U},\left.\mathcal{D}(\mathbb{B})\right|_{U}\right)$ to $\mathrm{H}^{1}\left(\mathcal{M}_{U},\left.\mathcal{D}_{\mathbb{R}}(\mathbb{B})\right|_{U}\right)$ where $U$ is an open subset of $B, \mathcal{M}_{U}:=\phi^{-1}(U)$ and $\mathcal{D}(\mathbb{B})$ (respectively $\mathcal{D}_{\mathbb{R}}(\mathbb{B})$ ) is the sheaf over $\mathcal{M}$ of germs of holomorphic sections of (respectively of germs of $C^{\infty}$ sections which are holomorphic along the fibers of) the vector bundle $(\mathbb{B}, \mathcal{M}, \pi)$. Let $\Theta:=\mathcal{D}(\mathbb{F})$ and $\Theta_{\mathbb{R}}:=\mathcal{D}_{\mathbb{R}}(\mathbb{F})$ where $\mathbb{F}$ is the sub-bundle, of the holomorphic tangent bundle $\mathbb{G}$ of $\mathcal{M}$, of tangent vectors along fibers of $\phi$. Hence for the special case $\mathbb{B}=\mathbb{F}$, we will be able to deduce the relationship between $\mathcal{H}^{1}(\Theta)$ and $\mathcal{H}^{1}\left(\Theta_{\mathbb{R}}\right)$.

This relationship is important because, while the global Kodaira-Spencer map for the complex analytic family $(\mathcal{M}, B, \phi)$ viz. $\rho: \Upsilon_{B} \longrightarrow \mathcal{H}^{1}(\Theta)$ described in (3.2.10) has target $\mathcal{H}^{1}(\Theta)$, the global Kodaira-Spencer map for the underlying differentiable family of $(\mathcal{M}, B, \phi)$, written as $\rho^{\mathbb{R}}: \Upsilon_{B_{\mathbb{R}}}^{\mathbb{R}} \longrightarrow$ $\mathcal{H}^{1}\left(\Theta_{\mathbb{R}}\right)$, has target $\mathcal{H}^{1}\left(\Theta_{\mathbb{R}}\right)$ where $B_{\mathbb{R}}$ denotes the underlying differentiable manifold of $B$ and $\Upsilon_{B_{\mathbb{R}}}^{\mathbb{R}}$ denotes the sheaf associated to the $C^{\infty}$ tangent bundle of $B_{\mathbb{R}}$.

Now consider the underlying differentiable family of $(\mathcal{M}, B, \phi)$. Then its fundamental sequence of vector bundles on $\mathcal{M}$ (see (3.2.3)) is given by

$$
0 \longrightarrow \mathbb{F}_{\mathbb{R}} \longrightarrow \mathbb{G}_{\mathbb{R}} \longrightarrow \mathbb{G}_{\mathbb{R}} / \mathbb{F}_{\mathbb{R}} \longrightarrow 0
$$

For any complex vector bundle $\mathbb{V}=(\mathbb{V}, \mathcal{M}, p)$ over $\mathcal{M}$, let $\overline{\mathbb{V}}$ denote the conjugate bundle of $\mathbb{V}, \mathbb{V}^{*}$ the dual bundle of $\mathbb{V}, \Lambda(\mathbb{V})$ the exterior algebra 
bundle of $\mathbb{V}$ and $\Lambda^{r}(\mathbb{V})$ the $r$-th graded piece of $\Lambda(\mathbb{V})$ called the $r$-th exterior power of $\mathbb{V}$. If $\mathbb{W}=(\mathbb{W}, \mathcal{M}, q)$ is another complex vector bundle over $\mathcal{M}$, $\mathbb{V} \Lambda \mathbb{W}$ denotes the exterior product of $\mathbb{V}$ and $\mathbb{W}$. With these notations, let

$$
\mathbb{F}_{\mathbb{R}}^{*}(r, s)=\left(\Lambda^{r}\left(\mathbb{F}_{\mathbb{R}}^{*}\right)\right) \Lambda\left(\Lambda^{s}\left(\overline{\mathbb{F}_{\mathbb{R}}^{*}}\right)\right) .
$$

The $C^{\infty}$ sections of the complex vector bundle $\mathbb{F}_{\mathbb{R}}^{*}(r, s)$ will be called differential forms of type $(r, s)$ and degree $(r+s)$ along the fibers of $\phi$. Let $A(r, s)$ be the space of differential forms of type $(r, s)$ along the fibers of $\phi$ and let $A:=\oplus_{(r, s)} A(r, s)$ be the space of differential forms of all degrees and types along the fibers of $\phi$. Then there exists a unique map $d_{\mathbb{R}}: A \longrightarrow A$ called exterior differentiation characterized by the following properties:

1. $d_{\mathbb{R}}$ is an anti-derivation of degree 1 ;

2. if $f: \mathcal{M} \longrightarrow \mathbb{C}$ is a complex-valued function on $\mathcal{M}$ (since functions can be considered naturally as forms of degree zero) then the form $d_{\mathbb{R}} f$ restricted to any fiber of $\phi$ must be the differential of the function $f$ restricted to that fiber of $\phi$ - in other words, $d_{\mathbb{R}} f$ is the gradient of $f$ along the fibers of $\phi$;

3. $d_{\mathbb{R}}^{2}=d_{\mathbb{R}} \circ d_{\mathbb{R}}=0$.

A map of $A$ into itself is said to be of type $(\mu, \nu)$ if, for each $(r, s)$, it maps $A(r, s)$ into $A(r+\mu, s+\nu)$. Then we have $d_{\mathbb{R}}=\partial_{\mathbb{R}}+\overline{\partial_{\mathbb{R}}}$ where $\partial_{\mathbb{R}}$ is of type $(1,0)$ and $\overline{\partial_{\mathbb{R}}}$ is its conjugate of type $(0,1)$.

Next consider $(\mathcal{M}, B, \phi)$ as a complex analytic family. Let

$$
0 \longrightarrow \mathbb{F} \longrightarrow \mathbb{G} \longrightarrow \mathbb{G} / \mathbb{F} \longrightarrow 0
$$

be the fundamental sequence of vector bundles on $\mathcal{M}$ for this family (see (3.2.10)). As we did for the underlying differentiable family above, we write

$$
\mathbb{G}^{*}(r, s)=\left(\Lambda^{r}\left(\mathbb{G}^{*}\right)\right) \Lambda\left(\Lambda^{s}\left(\overline{\mathbb{G}^{*}}\right)\right)
$$

and similarly

$$
\mathbb{F}^{*}(r, s)=\left(\Lambda^{r}\left(\mathbb{F}^{*}\right)\right) \Lambda\left(\Lambda^{s}\left(\overline{\mathbb{F}^{*}}\right)\right) .
$$

The differentiable sections of $\mathbb{G}^{*}(r, s)$ will be called differential forms of type $(r, s)$ on $\mathcal{M}$, while the differentiable sections of $\mathbb{F}^{*}(r, s)$ will be called differential forms of type $(r, s)$ along the fibers of $\phi$. Similarly, if $\mathbb{B}=(\mathbb{B}, \mathcal{M}, \pi)$ is a complex-analytic vector bundle on $\mathcal{M}$, then the differentiable sections of $\mathbb{B} \otimes \mathbb{G}^{*}(r, s)$ and $\mathbb{B} \otimes \mathbb{F}^{*}(r, s)$ will be called respectively $\mathbb{B}$-forms of type $(r, s)$ and $\mathbb{B}$-forms of type $(r, s)$ along the fibers of $\phi$.

Dualizing the fundamental sequence of vector bundles for the complex analytic family in consideration, we get the following short exact sequence of complex vector bundles on $\mathcal{M}$ :

$$
0 \longrightarrow(\mathbb{G} / \mathbb{F})^{*} \longrightarrow \mathbb{G}^{*} \longrightarrow \mathbb{F}^{*} \longrightarrow 0
$$


from which we conclude that there is a natural projection $F(r, s)$ of the space of differential forms of type $(r, s)$ onto the space of differential forms of type $(r, s)$ along the fibers of $\phi$. Taking the direct sum over all $(r, s)$, we obtain a projection $F$ of forms on $\mathcal{M}$ onto the space of forms along the fibers of $\phi$. Given any complex-analytic vector bundle $\mathbb{B}=(\mathbb{B}, \mathcal{M}, \pi)$, this induces a projection, which we also denote by $F$, of the space of $\mathbb{B}$-forms onto the space of $\mathbb{B}$-forms along the fibers of $\phi$.

The exterior differential operator $d$ operating on the differential forms on $\mathcal{M}$ splits in the usual way, namely $d=\partial+\bar{\partial}$ where $\partial$ is an operator of type $(1,0)$ and $\bar{\partial}$ is the conjugate operator of type $(0,1)$. Further $\partial$ and $\bar{\partial}$ also operate on $\mathbb{B}$-forms on $\mathcal{M}$.

We then have $F \circ \bar{\partial}=\overline{\partial_{\mathbb{R}}} \circ F$.

Let $\mathcal{D}(\mathbb{B})$ be the sheaf over $\mathcal{M}$ of germs $\zeta$ of $\mathbb{B}$-forms of type $(0,1)$ satisfying $\bar{\partial} \zeta=F \zeta=0$. Now let $\psi$ be a germ of $\mathcal{D}_{\mathbb{R}}(\mathbb{B})$. Then we have $F \bar{\partial} \psi=\overline{\partial_{\mathbb{R}}} F \psi=0$. Thus $\bar{\partial} \psi \in \mathcal{D}(\mathbb{B})$. Conversely, if $\zeta \in \mathcal{D}(\mathbb{B})$, then there exists a germ $\psi$ of a $C^{\infty}$ section of $\mathbb{B}$ such that $\bar{\partial} \psi=\zeta$. Now we have $\overline{\partial_{\mathbb{R}}} F \psi=F \bar{\partial} \psi=F \zeta=0$ which means that $\psi \in \mathcal{D}_{\mathbb{R}}(\mathbb{B})$. Further this $\psi$ belongs to the subsheaf $\mathcal{D}(\mathbb{B})$ if and only if $\bar{\partial} \psi=0$. Hence we have the following short exact sequence of sheaves on $\mathcal{M}$ :

$$
0 \longrightarrow \mathcal{D}(\mathbb{B}) \stackrel{i}{\longrightarrow} \mathcal{D}_{\mathbb{R}}(\mathbb{B}) \stackrel{\bar{\partial}}{\longrightarrow} \mathcal{D}(\mathbb{B}) \longrightarrow 0 .
$$

Here $i$ is the natural inclusion map. Let $U$ be an open subset of $B$ and $\mathcal{M}_{U}:=\phi^{-1}(U)$. From the short exact sequence obtained from the above short exact sequence by restricting to $U$, we obtain the following corresponding long exact sequence of cohomology groups (see (A.8))

$$
\begin{aligned}
& \cdots \longrightarrow \mathrm{H}^{0}\left(\mathcal{M}_{U},\left.\mathcal{D}_{\mathbb{R}}(\mathbb{B})\right|_{\mathcal{M}_{U}}\right) \stackrel{\bar{\partial}_{U}}{\longrightarrow} \mathrm{H}^{0}\left(\mathcal{M}_{U},\left.\mathcal{D}(\mathbb{B})\right|_{\mathcal{M}_{U}}\right) \stackrel{\delta_{U}^{*}}{\longrightarrow} \\
& \quad \stackrel{\delta_{U}^{*}}{\longrightarrow} \mathrm{H}^{1}\left(\mathcal{M}_{U},\left.\mathcal{D}(\mathbb{B})\right|_{\mathcal{M}_{U}}\right) \stackrel{i_{U}^{*}}{\longrightarrow} \mathrm{H}^{1}\left(\mathcal{M}_{U},\left.\mathcal{D}_{\mathbb{R}}(\mathbb{B})\right|_{\mathcal{M}_{U}}\right) \longrightarrow \cdots .
\end{aligned}
$$

Now take $\mathbb{B}=\mathbb{F}$, vary $U$ in $B$ and sheafify the presheaves defined by

$$
U \mapsto \mathrm{H}^{1}\left(\mathcal{M}_{U},\left.\mathcal{D}(\mathbb{F})\right|_{\mathcal{M}_{U}}\right) \text { and } U \mapsto \mathrm{H}^{1}\left(\mathcal{M}_{U},\left.\mathcal{D}_{\mathbb{R}}(\mathbb{F})\right|_{\mathcal{M}_{U}}\right)
$$

The maps $i_{U}^{*}$ of the long exact sequence above, as $U$ varies, define a homomorphism of sheaves $i^{*}: \mathcal{H}^{1}(\Theta) \longrightarrow \mathcal{H}^{1}\left(\Theta_{R}\right)$.

Now we have the following global analogue of the result obtained in (3.2.9) relating the global Kodaira-Spencer map for a complex analytic family to the global Kodaira-Spencer map for the underlying differentiable family.

3.2.11.1 Theorem. Let $(\mathcal{M}, B, \phi)$ be a complex analytic family of compact complex manifolds and let the global Kodaira-Spencer map for the 
underlying differentiable family be denoted by $\rho^{\mathbb{R}}: \Upsilon_{B_{\mathbb{R}}}^{\mathbb{R}} \longrightarrow \mathcal{H}^{1}\left(\Theta_{\mathbb{R}}\right)$ where $B_{\mathbb{R}}$ denotes the underlying differentiable manifold of $B, \Upsilon_{B_{\mathbb{R}}}^{\mathbb{R}}$ denotes the sheaf associated to its $C^{\infty}$ tangent bundle. Let $\Upsilon_{B_{\mathbb{R}}}^{\mathbb{R}} \otimes_{\mathbb{R}} \mathbb{C}$ be the sheaf associated to the complexification of this $C^{\infty}$ tangent bundle. Then we have the following commutative diagram:

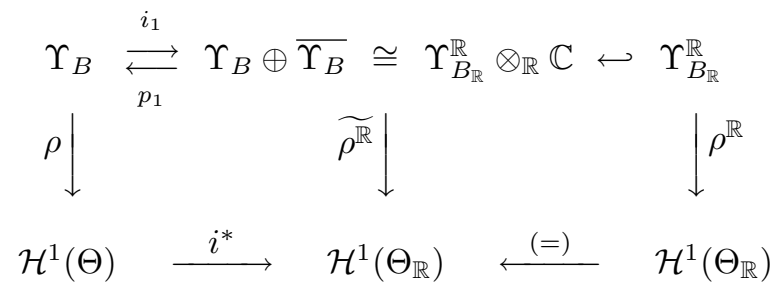

Recall that if a complex analytic family is such that its underlying differentiable family is locally trivial, then the family is itself (complex analytically) locally trivial. This was proved in (3.2.9.1). We obtain the same result below, but this time using the global Kodaira-Spencer maps. First of all, we state some deep results which have analogues in the category of schemes.

Let $\mathbb{B}_{t}$ be the restriction of a complex-analytic vector bundle $(\mathbb{B}, \mathcal{M}, \pi)$ on $\mathcal{M}$ to $M_{t}=\phi^{-1}(t), t \in B$ and $\Omega\left(\mathbb{B}_{t}\right)$ be the sheaf over $M_{t}$ of germs of holomorphic sections of $\mathbb{B}_{t}$ over $M_{t}$. Now we state the following nontrivial results. For proofs, refer to the paper of Kodaira-Spencer [6], chap. I, sec. 2.

\subsubsection{Theorem.}

1. (Semicontinuity Principle) For each nonnegative integer $q$ the function on $B$ that associates to $t \in B$ the dimension of the vector space $H^{q}\left(M_{t}, \Omega\left(\mathbb{B}_{t}\right)\right)$ is an upper semicontinuous function.

2. (Cohomology and Base Change) If for each $t \in B$ the dimension of the vector space $H^{q}\left(M_{t}, \Omega\left(\mathbb{B}_{t}\right)\right)$ is the same, then the natural restriction map

$$
H^{k}(\mathcal{M}, \mathcal{D}(\mathbb{B})) \longrightarrow H^{k}\left(M_{t}, \Omega\left(\mathbb{B}_{t}\right)\right)
$$

is surjective for $k=q$ and $k=q-1$.

3. If for each $t \in B$ the dimension of the vector space $H^{1}\left(M_{t}, \Omega\left(\mathbb{B}_{t}\right)\right)$ is the same, then $\cup_{t \in B} H^{1}\left(M_{t}, \Omega\left(\mathbb{B}_{t}\right)\right.$ ) forms (the total space of) a differentiable complex vector bundle over $B$ in a canonical manner whose sheaf of germs of $C^{\infty}$ sections is isomorphic to $\mathcal{H}^{1}(\mathcal{D}(\mathbb{B}))$.

4. If for each $t \in B$ the dimension of the vector space $H^{0}\left(M_{t}, \Omega\left(\mathbb{B}_{t}\right)\right)$ is the same, then for each $t \in B$, the homomorphism

$$
i_{U}^{*}: H^{1}\left(\mathcal{M}_{U},\left.\mathcal{D}(\mathbb{B})\right|_{\mathcal{M}_{U}}\right) \longrightarrow H^{1}\left(\mathcal{M}_{U},\left.\mathcal{D}_{\mathbb{R}}(\mathbb{B})\right|_{\mathcal{M}_{U}}\right)
$$


is injective for a sufficiently small spherical local complex coordinate neighborhood $U$ of $t$.

Now we use the above theorem to prove the following.

3.2.11.3 Theorem. If the underlying differentiable family of a complex analytic family $(\mathcal{M}, B, \phi)$ of compact complex manifolds is locally trivial, then the complex analytic family is itself (i.e., complex analytically) locally trivial.

Proof. By assertion (2) of theorem (3.2.10.1) it is enough to prove that the global Kodaira-Spencer map $\rho: \Upsilon_{B} \longrightarrow \mathcal{H}^{1}(\Theta)$ vanishes. Since the underlying differentiable family is locally trivial, all the fibers of this family are biholomorphic to each other. Hence the dimension of the vector space $\mathrm{H}^{0}\left(M_{t}, \Theta_{t}\right)$ is independent of $t \in B$. Now applying (4) of the previous theorem to $\mathbb{B}=\mathbb{F}$, we see that $i_{U}^{*}$ is always injective for a sufficiently small spherical local complex coordinate neighborhood $U$ of $t$ and this holds for each $t \in B$. Hence the map $i^{*}: \mathcal{H}^{1}(\Theta) \longrightarrow \mathcal{H}^{1}\left(\Theta_{\mathbb{R}}\right)$ is injective. By hypothesis we also know (from assertion (2) of theorem (3.2.7.1)) that the global Kodaira-Spencer map for the underlying differentiable family vanishes. Now looking at the commutative diagram in theorem (3.2.11.1) we conclude (since $i^{*}$ is injective and $\widetilde{\rho^{\mathbb{R}}}$ is the zero homomorphism) that $\rho$ is the zero homomorphism QED

3.2.11.4 Important Remarks. The notion of deformations of a complex analytic space parametrized by another complex analytic space is a generalization of the notion of complex analytic family (see (3.6.1.1)). This notion was made precise by Grauert who in [11] extended all the results of Kodaira-Spencer mentioned in this section. Further Grauert alongwith Fischer proved in [12] the following nice result.

If a complex analytic family of compact complex manifolds has all its fibers biholomorphic to each other, then this family must be locally (complex analytically) trivial. 


\subsection{Behaviour of Infinitesimal Kodaira- Spencer Maps Under Change of the Parameter}

The results of this section apply to both complex analytic families and differentiable families. So we omit adjectives and simply use the words "family", "map", etc., instead of "complex analytic family", "holomorphic map" and so on (or "differentiable family", " $C^{\infty}$ map", etc.,), always assuming that we have either the complex analytic category or the differentiable category fixed for the discussion below.

\subsection{1 "Chain Rule" for the Derivative of Complex Structure}

Let $(\mathcal{M}, B, \phi)=\left\{M_{t}:=\phi^{-1}(t) ; t \in B\right\}$ be a family of compact complex manifolds. Let $h: D \longrightarrow B$ be a morphism of manifolds. Then (by (2.4.3.3) and (2.2.3.3) respectively for the complex analytic category and the differentiable category) we have the induced family $\left(h^{*} \mathcal{M}, D, h^{*} \phi\right)$. Recall that if $h(s)=t$, then $\left(h^{*} \phi\right)^{-1}(s)$ is (biholomorphic to) $M_{t}=M_{h(s)}$ and hence we may write $\left(h^{*} \mathcal{M}, D, h^{*} \phi\right)=\left\{M_{h(s)} ; s \in D\right\}$.

We now wish to relate the local deformations of $M_{t_{0}}$ in a neighborhood of $t_{0}$ (for a fixed $t_{0} \in B$ ) to the local deformations of $M_{h\left(s_{0}\right)}$ in a neighborhood of $s_{0}$ (where $\left.h\left(s_{0}\right)=t_{0}\right)$. Recall (from theorem (2.3.2.4)) that there exists a sufficiently small coordinate polydisc $I \subset B$ with $t_{0} \in I$ such that the restricted family $\left(\mathcal{M}_{I}, I, \phi\right)$ has deformation space

$$
\mathcal{M}_{I}=\left(\cup_{j=1}^{l}\left(V_{j} \times I\right)\right) / \sim_{I}
$$

where $V_{1}, \ldots, V_{l}$ are the polydiscs which are glued via the transition functions $\left\{f_{j k}\left(z_{k}, t_{0}\right)\right\}$ to give the compact complex manifold $M_{t_{0}}$ and where the equivalence relation $\sim_{I}$ is defined thus: if $\left(z_{j}, t\right) \in V_{j} \times I$ and $\left(z_{k}, t^{\prime}\right) \in V_{k} \times I$ then $\left(z_{j}, t\right) \sim_{I}\left(z_{k}, t^{\prime}\right)$ if and only if $t=t^{\prime}$ and $z_{j}=f_{j k}\left(z_{k}, t\right)$.

Choose a polydisc (i.e., a local coordinate neighborhood) $J \subset D$ with $s_{0} \in J$ such that $h(J) \subset I$ and consider the restriction of the induced family $\left(h^{*} \mathcal{M}, D, h^{*} \phi\right)$ to $J$. We may identify $J$ with the graph $G_{J}:=$ $\{(h(s), s) ; s \in J\}$ of $h$ and hence by the construction of (2.4.3.3) or (2.2.3.3) we get the deformation space of this restricted family as

$$
\left(h^{*} \mathcal{M}\right)_{J}=\left(\cup_{j=1}^{l}\left(V_{j} \times G_{J}\right)\right) / \sim_{J}
$$

where the equivalence relation $\sim_{J}$ is defined as follows: if $\left(z_{j}, h(s), s\right) \in$ $V_{j} \times G_{J},\left(z_{k}, h\left(s^{\prime}\right), s^{\prime}\right) \in V_{k} \times G_{J}$ then $\left(z_{j}, h(s), s\right) \sim_{J}\left(z_{k}, h\left(s^{\prime}\right), s^{\prime}\right)$ if and 
only if $s=s^{\prime}$ and $z_{j}=f_{j k}\left(z_{k}, h(s)\right)$. Again identifying $G_{J}$ with $J$, we may rewrite the above as follows:

$$
\left(h^{*} \mathcal{M}\right)_{J}=\left(\cup_{j=1}^{l}\left(V_{j} \times J\right)\right) / \sim_{J}
$$

which shows that the restricted family $\left(\left(h^{*} \mathcal{M}\right)_{J}, J, h^{*} \phi\right)$ is simply obtained by glueing $V_{1} \times J, \ldots, V_{l} \times J$ via the transition functions got by formally substituting $t=h(s)$ in the transition functions that define $\mathcal{M}_{I}$.

In this sense we see that the glueing data on $\left(h^{*} \mathcal{M}, D, h^{*} \phi\right)$ are locally actually governed by the glueing data locally on $(\mathcal{M}, B, \phi)$ and hence it is justified to say that the variation of complex structures in the family $\left(h^{*} \mathcal{M}, D, h^{*} \phi\right)$ is induced locally by the variation of complex structures in $(\mathcal{M}, B, \phi)$.

Recall from (3.2.1.1) or (3.2.8.1) that for a tangent vector $\frac{\partial}{\partial t} \in T_{t} B$ $(t \in B)$, the infinitesimal deformation of $M_{t}$ was defined as $\rho_{t}\left(\frac{\partial}{\partial t}\right)=\frac{\partial M_{t}}{\partial t}=$ $\theta(t)$. We called it the derivative of the complex structure of $M_{t}$ along the direction prescribed by the tangent vector $\frac{\partial}{\partial t}$. We know from Calculus that derivatives satisfy the chain rule: if $t=h(s)$ and if $\frac{\partial}{\partial t}=\sum_{\lambda} \frac{\partial}{\partial t_{\lambda}}$, then we have $\frac{\partial}{\partial s}=\sum_{\lambda} \frac{\partial t_{\lambda}}{\partial s} \frac{\partial}{\partial t_{\lambda}}$. An analogous property is satisfied by infinitesimal deformations and hence an infinitesimal deformation may be regarded as a "derivative" measuring the "instantaneous rate of change of complex structure with respect to the parameter". More precisely we have the following.

3.3.1.1 Theorem (Chain Rule). Let $(\mathcal{M}, B, \phi)=\left\{M_{t}:=\phi^{-1}(t) ; t \in\right.$ $B\}$ be a family of compact complex manifolds and $h: D \longrightarrow B$ be a morphism of manifolds. Let $\left(h^{*} \mathcal{M}, D, h^{*} \phi\right)=\left\{M_{h(s)} ; s \in D\right\}$ be the family induced by $h$ and $s_{0} \in D$ such that $h\left(s_{0}\right)=t_{0} \in B$. Then for any tangent vector $(\partial / \partial s) \in T_{s_{0}} D$, the infinitesimal deformation (or derivative of complex structure) of $M_{h\left(s_{0}\right)}$ along $\frac{\partial}{\partial s}$ is given by the "chain rule":

$$
\rho_{s_{0}}\left(\frac{\partial}{\partial s}\right)=\frac{\partial M_{h\left(s_{0}\right)}}{\partial s}=\sum_{\lambda=1}^{m} \frac{\partial t_{\lambda}}{\partial s} \frac{\partial M_{t_{0}}}{\partial t_{\lambda}}
$$

where $\frac{\partial}{\partial t}=\sum_{\lambda=1}^{m} \frac{\partial}{\partial t_{\lambda}} \in T_{t_{0}} B$ is the image of $\frac{\partial}{\partial s}$ under the differential $d h_{s_{0}}: T_{s_{0}} D \longrightarrow T_{t_{0}} B$ and $t=\left(t_{1}, \ldots, t_{m}\right)$ are local coordinates at $t_{0}$. Hence we also have the following commutative diagram:

$$
\begin{aligned}
& H^{1}\left(M_{h\left(s_{0}\right)}, \Theta_{h\left(s_{0}\right)}\right) \stackrel{(=)}{\longrightarrow} H^{1}\left(M_{t_{0}}, \Theta_{t_{0}}\right) \\
& \rho_{s_{0}} \uparrow \quad \uparrow \rho_{t_{0}} \\
& T_{s_{0}} D \stackrel{d h_{s_{0}}}{\longrightarrow} \quad T_{t_{0}} B
\end{aligned}
$$


corresponding to the pullback (or fiber product) commutative diagram:

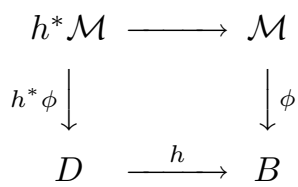

Proof. In view of the discussion on the induced family preceding the statement of this theorem, this is a straightforward verification QED

\subsubsection{Indispensability of the Assumption of Regularity}

Recall the construction in $(2.8 .2)$ of a family $(\mathcal{M}, \mathbb{C}, \phi)$ of Hopf surfaces exhibiting the jump phenomenon at $0 \in \mathbb{C}$. Note that this family restricted to $U:=\mathbb{C}-\{0\}$ is actually trivial but the fiber $M_{0}$ over $0 \in \mathbb{C}$ is not biholomorphic to any other fiber of this family. Consider the map $h: \mathbb{C} \longrightarrow$ $\mathbb{C}$ given by $t=h(s)=s^{2}$. For simplicity, we denote the induced family $\left(h^{*} \mathcal{M}, \mathbb{C}, h^{*} \phi\right)$ by $(\mathcal{N}, \mathbb{C}, \pi)$. By the theorem of the previous subsection, the infinitesimal deformation along $\frac{d}{d s} \in T_{s} \mathbb{C}$ of $\pi^{-1}(s)=M_{s^{2}}$ is given by

$$
\rho_{s}\left(\frac{d}{d s}\right)=\frac{d M_{s^{2}}}{d s}=\frac{d t}{d s} \frac{d M_{t}}{d t}=2 s \frac{d M_{t}}{d t} .
$$

If $s \neq 0, t=s^{2} \in U=\mathbb{C}-\{0\}$ and since $(\mathcal{M}, \mathbb{C}, \phi)$ restricted to $U$ is trivial, we have $\frac{d M_{t}}{d t}=0$ and hence $\rho_{s}$ vanishes identically for $s \neq 0$; further $\rho_{0}$ also vanishes due to the above computation.

Hence the infinitesimal Kodaira-Spencer map at each point of the base vanishes for the induced family $(\mathcal{N}, \mathbb{C}, \pi)$. However, this family is not locally trivial since the fiber over 0 is not biholomorphic to any other fiber by construction.

Hence by theorem (3.2.7.3) or by assertion (2) of theorem (3.2.8.3), this induced family cannot be a regular family. Note however that the restriction of this family to $U$ is regular! Explicit computations by Kodaira-Spencer ([6], chap. VI, sec. 15) verify that this is indeed the case; in fact, their results are:

$$
\operatorname{dim}\left(\mathrm{H}^{1}\left(M_{t}, \Theta_{t}\right)\right)= \begin{cases}4, & t=0 \\ 2, & t \neq 0 .\end{cases}
$$

Recall that in (2.8.2) we mentioned that

$$
\operatorname{dim}\left(\mathrm{H}^{0}\left(M_{t}, \Theta_{t}\right)\right)= \begin{cases}4, & t=0 \\ 2, & t \neq 0\end{cases}
$$


and Kodaira-Spencer show that $\operatorname{dim}\left(\mathrm{H}^{0}\left(M_{t}, \Theta_{t}\right)\right)=\operatorname{dim}\left(\mathrm{H}^{1}\left(M_{t}, \Theta_{t}\right)\right)$ for each $t \in \mathbb{C}$.

This example shows that the additional hypothesis of regularity, to conclude local triviality from the vanishing of the infinitesimal Kodaira-Spencer maps at each point of the parameter space for a family of compact complex manifolds, is indispensable.

\subsection{The Theorems of Completeness and Existence}

Let $M$ be a compact complex manifold and $(\mathcal{M}, B, \phi)$ be a complex analytic family of compact complex manifolds with $t_{0} \in B$ such that $M_{t_{0}}:=\phi^{-1}\left(t_{0}\right)$ is biholomorphic to $M$. We then have the infinitesimal Kodaira-Spencer map $\rho_{t_{0}}: T_{t_{0}} B \longrightarrow \mathrm{H}^{1}(M, \Theta)$ which associates to every tangent vector $\frac{\partial}{\partial t}$ at $t_{0}$ to $B$, a cohomology class called the infinitesimal deformation of $M_{t_{0}}$ along $\frac{\partial}{\partial t}$, written as

$$
\theta(t)=\rho_{t_{0}}\left(\frac{\partial}{\partial t}\right)=\frac{\partial M}{\partial t} \in \mathrm{H}^{1}(M, \Theta) .
$$

The infinitesimal deformations are thus certain elements of $\mathrm{H}^{1}(M, \Theta)$. The question that naturally arises is whether each element $\theta \in \mathrm{H}^{1}(M, \Theta)$ is an infinitesimal deformation of $M$ i.e., given such a $\theta$, does there exist a complex analytic family $(\mathcal{M}, B, \phi)$ with embedded fiber $M_{t_{0}}:=\phi^{-1}\left(t_{0}\right)$ biholomorphic to $M$ (for some $t_{0} \in B$ ) and a tangent vector $\frac{\partial}{\partial t}$ at $t_{0}$ to $B$ along whose direction the infinitesimal deformation of $M$ is precisely $\theta$ ?

It is not clear that each $\theta$ should arise in this way. It turns out that if $\theta$ arises in this way, then it has to satisfy certain additional conditions. Thus if there exist cohomology classes $\theta$ which do not satisfy these additional conditions, then obviously such $\theta$ are not infinitesimal deformations of $M$ and these are called "obstructions to deformation of $M$ ". This leads to "obstruction theory" which we describe below.

\subsubsection{Primary Obstructions to Infinitesimal Deformations}

Let $M$ be a compact complex manifold and let $(\mathcal{M}, B, \phi)$ be a complex analytic family with $0 \in B \subset \mathbb{C}^{m}$ such that $M_{0}:=\phi^{-1}(0)$ is biholomorphic to $M$. Taking a small local complex coordinate neighborhood $\Delta$ of $0 \in B$ contained in $B$ (which is biholomorphic to a polydisc), we know that we may write

$$
\phi^{-1}(\Delta)=\mathcal{M}_{\Delta}=\left(\cup_{j=1}^{l}\left(U_{j} \times \Delta\right)\right) / \sim_{\Delta}
$$


where $U_{1}, \ldots, U_{l}$ are the polydiscs which are glued via the transition functions $\left\{f_{j k}\left(z_{k}, 0\right)\right\}$ to give the compact complex manifold $M_{0} \cong M$ and the equivalence relation $\sim_{\Delta}$ is defined thus: if $\left(z_{j}, t\right) \in U_{j} \times \Delta$ and $\left(z_{k}, t^{\prime}\right) \in$ $U_{k} \times \Delta$ then $\left(z_{j}, t\right) \sim_{\Delta}\left(z_{k}, t^{\prime}\right)$ if and only if $t=t^{\prime}$ and $z_{j}=f_{j k}\left(z_{k}, t\right)$. Each transition function $f_{j k}$ is holomorphic in $z_{k}$ as well as in $t=\left(t_{1}, \ldots, t_{m}\right) \in$ $B \subset \mathbb{C}^{m}$ and is defined on the nonempty intersection $\left(U_{j} \times \Delta\right) \cap\left(U_{k} \times \Delta\right)$.

Recall that if $\frac{\partial}{\partial t} \in T_{t} B$ is a tangent vector, we have

$$
\theta(t)=\rho_{t}\left(\frac{\partial}{\partial t}\right)=\frac{\partial M_{t}}{\partial t} \in \mathrm{H}^{1}\left(M_{t}, \Theta_{t}\right)
$$

where $\theta(t)$ is obtained from the 1-cocycle $\left\{\theta_{j k}(t)\right\} \in Z^{1}\left(\mathcal{U}_{t}, \Theta_{t}\right)\left(\mathcal{U}_{t}:=\left\{U_{j} \times\right.\right.$ $\{t\} ; j=1, \ldots, l\})$ and the holomorphic vector fields $\theta_{j k}$ are given by

$$
\theta_{j k}\left(z_{j}, t\right)=\sum_{\alpha=1}^{n} \frac{\partial f_{j k}^{\alpha}\left(z_{k}, t\right)}{\partial t} \frac{\partial}{\partial z_{j}^{\alpha}}, z_{k}=f_{k j}\left(z_{j}, t\right)
$$

where $n$ is the dimension of $M$ as a complex manifold. Further, on $\left(U_{i} \times\right.$ $\Delta) \cap\left(U_{j} \times \Delta\right) \cap\left(U_{k} \times \Delta\right) \neq \emptyset$ we have the patching conditions

$$
f_{i k}^{\alpha}\left(z_{k}, t\right)=f_{i j}^{\alpha}\left(f_{j k}\left(z_{k}, t\right), t\right) \quad(1 \leq \alpha \leq n) .
$$

Differentiating along $\frac{\partial}{\partial t}$ we get the cocycle condition on $\left\{\theta_{j k}(t)\right\}$ namely:

$$
\theta_{i k}^{\alpha}\left(z_{i}, t\right)=\theta_{i j}^{\alpha}\left(z_{i}, t\right)+\sum_{\beta=1}^{n} \frac{\partial z_{i}^{\alpha}}{\partial z_{j}^{\beta}} \theta_{j k}^{\beta}\left(z_{j}, t\right)(1 \leq \alpha \leq n)
$$

where we have

$$
\theta_{j k}^{\alpha}\left(z_{j}, t\right)=\frac{\partial f_{j k}^{\alpha}\left(z_{k}, t\right)}{\partial t}, z_{k}=f_{k j}\left(z_{j}, t\right)
$$

Now for any two given local holomorphic vector fields on $M_{t}$ over the open subset $U_{j} \times\{t\}$ of the form

$$
v=\sum_{\alpha=1}^{n} v_{j}^{\alpha}\left(\frac{\partial}{\partial z_{j}^{\alpha}}\right), u=\sum_{\alpha=1}^{n} u_{j}^{\alpha}\left(\frac{\partial}{\partial z_{j}^{\alpha}}\right)
$$

we define the Poisson bracket $[v, u]$ by the formula:

$$
[v, u]=\sum_{\alpha=1}^{n}\left(v \cdot u_{j}^{\alpha}-u \cdot v_{j}^{\alpha}\right) \frac{\partial}{\partial z_{j}^{\alpha}} .
$$


Then $[v, u]$ has the following properties:

1. $[v, u]$ does not depend on the choice of local coordinates $\left(z_{j}^{1}, \ldots, z_{j}^{n}\right)$;

2. $[v, u]$ is bilinear in $u$ and $v$;

3. $[v, u]=-[u, v]$.

Now set

$$
\dot{\theta}_{j k}^{\alpha}\left(z_{j}, t\right)=\frac{\partial \theta_{j k}^{\alpha}\left(z_{j}, t\right)}{\partial t}, \dot{\theta}_{j k}(t)=\sum_{\alpha=1}^{n} \dot{\theta}_{j k}^{\alpha}\left(z_{j}, t\right) \frac{\partial}{\partial z_{j}^{\alpha}} .
$$

Then differentiating the cocycle conditions on $\left\{\theta_{j k}(t)\right\}$ totally with respect to $t$ along $\frac{\partial}{\partial t}$ and using the Poisson bracket defined above, we get the following set of important relations:

$$
\dot{\theta}_{i j}(t)-\dot{\theta}_{i k}(t)+\dot{\theta}_{j k}(t)=\left[\theta_{i j}(t), \theta_{j k}(t)\right] .
$$

Now for any 1-cocycle $\left\{\psi_{j k}(t)\right\} \in Z^{1}\left(\mathcal{U}_{t}, \Theta_{t}\right)$ we define

$$
\zeta_{i j k}(t)=\left[\psi_{i j}(t), \psi_{j k}(t)\right] \text { if }\left(U_{i} \times\{t\}\right) \cap\left(U_{j} \times\{t\}\right) \cap\left(U_{k} \times\{t\}\right) \neq \emptyset .
$$

Then $\zeta_{i j k}(t)$ is a holomorphic vector field on $\left(U_{i} \times\{t\}\right) \cap\left(U_{j} \times\{t\}\right) \cap\left(U_{k} \times\right.$ $\{t\}) \neq \emptyset$ and in fact, direct calculations reveal that $\left\{\zeta_{i j k}(t)\right\}$ is actually a 2-cocycle i.e., $\left\{\zeta_{i j k}(t)\right\} \in Z^{2}\left(\mathcal{U}_{t}, \Theta_{t}\right)$ (see (A.9)).

In fact, we can extend the above definition of $\left\{\zeta_{i j k}(t)\right\}$ to the case when we are given not only a single 1-cocycle $\left\{\psi_{j k}(t)\right\}$ but also another 1-cocycle $\left\{\eta_{j k}(t)\right\}$ by setting

$$
\zeta_{i j k}(t)=\frac{1}{2}\left(\left[\psi_{i j}(t), \eta_{j k}(t)\right]+\left[\eta_{i j}(t), \psi_{j k}(t)\right]\right)
$$

so that we have again that $\left\{\zeta_{i j k}(t)\right\} \in Z^{2}\left(\mathcal{U}_{t}, \Theta_{t}\right)$.

Let $\psi(t), \eta(t), \zeta(t)$ be the cohomology classes determined respectively by the 1-cocycles $\left\{\psi_{j k}(t)\right\},\left\{\eta_{j k}(t)\right\} \in Z^{1}\left(\mathcal{U}_{t}, \Theta_{t}\right)$ and the 2-cocycle $\left\{\zeta_{i j k}(t)\right\} \in$ $Z^{2}\left(\mathcal{U}_{t}, \Theta_{t}\right)$. Thus $\psi(t), \eta(t) \in \mathrm{H}^{1}\left(M_{t}, \Theta_{t}\right)$ whereas $\zeta(t) \in \mathrm{H}^{2}\left(M_{t}, \Theta_{t}\right)$.

We now extend the bracket to cohomology: $[\psi(t), \eta(t)]:=\zeta(t)$. Then it is routinely checked that this bracket is well-defined, bilinear in $\psi(t)$ and $\eta(t)$ and further satisfies anti-commutativity: $[\psi(t), \eta(t)]=-[\eta(t), \psi(t)]$. Note that we have defined a bilinear map:

$$
[-,-]: \mathrm{H}^{1}\left(M_{t}, \Theta_{t}\right) \times \mathrm{H}^{1}\left(M_{t}, \Theta_{t}\right) \longrightarrow \mathrm{H}^{2}\left(M_{t}, \Theta_{t}\right) .
$$

We can say more about this bilinear map. Let the short exact sequence of sheaves on $\mathcal{M}$ given by

$$
0 \longrightarrow \Theta \longrightarrow \Pi \stackrel{j}{\longrightarrow} \Upsilon \longrightarrow 0
$$


be the fundamental sequence of sheaves for the complex analytic family $(\mathcal{M}, B, \phi)$ (see (3.2.10)). Then $\Theta$ is a sheaf of germs of Lie algebras over the sheaf $\mathcal{O}_{\mathbb{C}}$ of germs of complex-valued differentiable functions on $\mathrm{M}$ which are constant along the fibers of $\phi$. Now a standard theorem from the Theory of Cohomology with Coefficients in Sheaves says that the direct sum $\mathcal{H}^{*}(\Theta):=\oplus_{q>0} \mathcal{H}^{q}(\Theta)$ is a sheaf of graded Lie algebras over $\mathcal{O}_{\mathbb{C}}$. Similarly, $\mathrm{H}^{*}\left(M_{t}, \Theta_{t}\right):=\oplus_{q \geq 0} \mathrm{H}^{q}\left(M_{t}, \Theta_{t}\right)$ is a graded Lie algebra over $\mathbb{C}$ for each $t \in B$. Further this graded Lie algebra structure is compatible with the natural restriction maps $\mathcal{H}^{q}(\Theta) \longrightarrow \mathrm{H}^{q}\left(M_{t}, \Theta_{t}\right)$. The bracket

$$
[\cdot, \cdot]: \mathrm{H}^{1}\left(M_{t}, \Theta_{t}\right) \times \mathrm{H}^{1}\left(M_{t}, \Theta_{t}\right) \longrightarrow \mathrm{H}^{2}\left(M_{t}, \Theta_{t}\right)
$$

that we have defined above is just the multiplication in the Lie algebra $\mathrm{H}^{*}\left(M_{t}, \Theta_{t}\right)$ restricted to $\mathrm{H}^{1}\left(M_{t}, \Theta_{t}\right)$.

Now if we set $\left\{\psi_{j k}(t)\right\}=\left\{\eta_{j k}(t)\right\}=\left\{\theta_{j k}(t)\right\}$ in the above calculations and further set $t=0$ we obtain

$$
\zeta_{i j k}(0)=\left[\theta_{i j}(0), \theta_{j k}(0)\right],\left\{\zeta_{i j k}(0)\right\} \mapsto \zeta(0) \in \mathrm{H}^{2}(M, \Theta) .
$$

If we set $t=0$ in the formula (3.4) above and replace $i$ by $k$ there then we obtain that

$$
-\dot{\theta}_{j k}(0)=\dot{\theta}_{k j}(0) \Rightarrow\left\{\dot{\theta}_{j k}(0)\right\} \in C^{1}\left(\mathcal{U}_{0}, \Theta_{0}\right)
$$

that is, $\left\{\dot{\theta}_{j k}(0)\right\}$ is a 1 -cochain with values in $\Theta_{0}=\Theta$ relative to the open cover $\mathcal{U}_{0}$ of $M_{0}=M$ (see (A.9)).

Further, the differential $\delta^{1}: C^{1}\left(\mathcal{U}_{0}, \Theta_{0}\right) \longrightarrow C^{2}\left(\mathcal{U}_{0}, \Theta_{0}\right)$ is defined (see (A.9)) by

$$
\delta^{1}\left(\left\{\dot{\theta}_{j k}(0)\right\}\right)=\left\{\tau_{i j k}(0)\right\} ; \tau_{i j k}(0):=\dot{\theta}_{j k}(0)-\dot{\theta}_{i k}(0)+\dot{\theta}_{i j}(0) .
$$

But then we see by the formula (3.4) above that $\tau_{i j k}(0)=\zeta_{i j k}(0)$ and hence $\left\{\zeta_{i j k}(0)\right\}$ is a coboundary (see $(\mathrm{A} .9)$ ) and hence $\zeta(0)=0$. But since we also have $\zeta(0)=[\theta(0), \theta(0)]$, we have proved the following.

3.4.1.1 Theorem. Let $M$ be a compact complex manifold and $\theta \in H^{1}(M, \Theta)$. If $M$ occurs as a fiber of a complex analytic family $(\mathcal{M}, B, \phi)$ of compact complex manifolds - say with $t_{0} \in B$ such that $M_{t_{0}}:=\phi^{-1}\left(t_{0}\right)$ is biholomorphic to $M$ - and if the infinitesimal deformation of $M$ along a certain direction prescribed by $\frac{\partial}{\partial t} \in T_{t_{0}} B$ is precisely $\theta$, then it is necessary that the element $[\theta, \theta] \in H^{2}(M, \Theta)$ vanishes.

Motivated by the above theorem we make the following definition.

3.4.1.2 Definition. Let $M$ be a compact complex manifold. An element $\theta \in \mathrm{H}^{1}(M, \Theta)$ is said to be obstructed if the corresponding element $[\theta, \theta] \in$ 
$\mathrm{H}^{2}(M, \Theta)$ is nonzero. If $\theta$ is obstructed, then the element $[\theta, \theta]$ is said to be a primary obstruction to infinitesimal deformation of complex structure of $M$.

The reason for the adjective "primary" in the above definition will be explained in (3.4.5) below.

Note that there do exist compact complex (even algebraizable) manifolds $M$ for which we can find an obstructed cohomology class $\theta \in \mathrm{H}^{1}(M, \Theta)$. Kodaira and Spencer in [6] give one such example: $M:=T^{n} \times P_{\mathbb{C}}^{1}$ where $T^{n}$ is a (possibly algebraic) $n$-dimensional complex torus, $n \geq 2$.

3.4.1.3 Remark. The statement of the above theorem (3.4.1.1) and its proof were discussed only for the case of a complex analytic family with $M$ embedded as one of the fibers of this family. However, it is clear that a similar theorem can be stated and proved as well for a differentiable family with $M$ embedded as one of the fibers there.

\subsubsection{Complete Families and the Theorem of Completeness}

Let $M$ be a compact complex manifold occurring as fiber of $\phi$ over $t_{0} \in B$ in a complex analytic family $(\mathcal{M}, B, \phi)$. We saw in the previous subsection that if $\theta \in \mathrm{H}^{1}(M, \Theta)$ is an infinitesimal deformation of $M$ i.e., if $\theta$ is in the image of the infinitesimal Kodaira-Spencer map $\rho_{t_{0}}: T_{t_{0}} B \longrightarrow \mathrm{H}^{1}(M, \Theta)$ then necessarily we must have that $[\theta, \theta]=0$ in $\mathrm{H}^{2}(M, \Theta)$. However, if $[\theta, \theta]=0$ for some $\theta \in \mathrm{H}^{1}(M, \Theta)$, we do not know if it is in the image of $\rho_{t_{0}}$ for some suitable complex analytic family $(\mathcal{M}, B, \phi)$ with $M=\phi^{-1}\left(t_{0}\right)$.

We could answer this question easily if we could find a complex analytic family $(\mathcal{M}, B, \phi)$ with $M=\phi^{-1}\left(t_{0}\right)$ and such that the infinitesimal Kodaira-Spencer map $\rho_{t_{0}}: T_{t_{0}} B \longrightarrow \mathrm{H}^{1}(M, \Theta)$ is surjective, for then it is obvious that every cohomology class arises from some infinitesimal deformation of $M$. It turns out that families with this property are special in the sense that they induce locally every infinitesimal deformation of $M$ in any complex analytic family. To make things more precise, we state the following.

3.4.2.1 Definition. Let $(\mathcal{M}, B, \phi)=\left\{M_{t}:=\phi^{-1}(t) ; t \in B\right\}$ be a complex analytic family of compact complex manifolds and let $t_{0}$ be a point of $B$. Let $(\mathcal{N}, D, \pi)$ be any complex analytic family of compact complex manifolds with $s_{0} \in D$ such that $N_{s_{0}}:=\pi^{-1}\left(s_{0}\right)$ is biholomorphic to $M_{t_{0}}$. Then we say that the complex analytic family $(\mathcal{M}, B, \phi)$ is complete at $t_{0} \in B$ if for each such complex analytic family $(\mathcal{N}, D, \pi)$, there is a sufficiently small neighborhood $E \subset D$ of $s_{0}$ and a holomorphic map $h: E \longrightarrow B$ such 
that $h\left(s_{0}\right)=t_{0}$ and the induced family $\left(h^{*} \mathcal{M}, E, h^{*} \phi\right)$ is equivalent to the restriction $\left(\mathcal{N}_{E}, E, \pi\right)$ of $(\mathcal{N}, D, \pi)$ to $E \subset D$. (The holomorphic map $h$ need not be unique.)

Hence we see that if $(\mathcal{M}, B, \phi)$ is complete at $t_{0} \in B$ then it contains all the infinitesimal deformations of $M_{t_{0}}=\phi^{-1}\left(t_{0}\right)$ - even those coming from arbitrary complex analytic families $(\mathcal{N}, D, \pi)$ with $N_{s_{0}}:=\pi^{-1}\left(s_{0}\right) \cong M_{t_{0}}$ and further also contains all sufficiently small local deformations of $M_{t_{0}}$ which may come from any arbitrary family $(\mathcal{N}, D, \pi)$ with $N_{s_{0}}:=\pi^{-1}\left(s_{0}\right) \cong$ $M_{t_{0}}$.

We state the following obvious extension of the above definition of completeness at a point to global completeness.

3.4.2.2 Definition. A complex analytic family of compact complex manifolds $(\mathcal{M}, B, \phi)$ is said to be (globally) complete if it is complete at each point $t \in B$.

Thus if $(\mathcal{M}, B, \phi)$ is complete, then it contains all the infinitesimal deformations of $M_{t}$ for each $t \in B$ and also all sufficiently small deformations of $M_{t}$ for each $t \in B$.

The next nontrivial theorem, due to Kodaira and Spencer [13], justifies what we said in the beginning of this subsection.

3.4.2.3 Theorem of Completeness. Let $(\mathcal{M}, B, \phi)$ be a complex analytic family of compact complex manifolds. Then $(\mathcal{M}, B, \phi)$ is complete at $t_{0} \in B$ if the infinitesimal Kodaira-Spencer map at $t_{0}$ viz. $\rho_{t_{0}}: T_{t_{0}} B \longrightarrow$ $H^{1}\left(M_{t_{0}}, \Theta_{t_{0}}\right)$ is surjective.

We will just indicate the philosophy behind the proof of the above theorem as given by Kodaira in his book [5], chap. 6 .

Let us first assume that $(\mathcal{M}, B, \phi)$ is a complex analytic family and $h: D \longrightarrow B$ a holomorphic map. Consider the induced complex analytic family $\left(h^{*} \mathcal{M}, D, h^{*} \phi\right)$. Then there is an obvious holomorphic map $g$ : $h^{*} \mathcal{M} \longrightarrow \mathcal{M}$ such that $g$ maps the compact complex manifold $\left(h^{*} \phi\right)^{-1}(s)$ biholomorphically onto $\phi^{-1}(h(s))=M_{h(s)}$ for each $s \in D$ i.e., the following diagram commutes (cf. (2.4.3.3)):

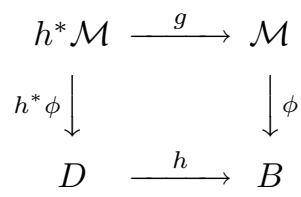

If we identify $\left(h^{*} \phi\right)^{-1}\left(s_{0}\right)=M_{h\left(s_{0}\right)} \times\left\{s_{0}\right\}$ with $M_{h\left(s_{0}\right)}=\phi^{-1}\left(h\left(s_{0}\right)\right)$ then we can say that $g$ extends the identity map on $M_{h\left(s_{0}\right)}$. The crucial first step in the proof of the theorem of completeness is the following simple converse. 
3.4.2.4 Lemma. Let $(\mathcal{M}, B, \phi)$ and $(\mathcal{N}, D, \pi)$ be complex analytic families of compact complex manifolds with $t_{0} \in B, s_{0} \in D$ such that $M=$ $\phi^{-1}\left(t_{0}\right)=\pi^{-1}\left(s_{0}\right)$. Suppose that we can find a sufficiently small neighborhood $\Delta$ of $s_{0}$ contained in D, a holomorphic map $h: \Delta \longrightarrow B$ with $h\left(s_{0}\right)=t_{0}$, and further a holomorphic map $g: \mathcal{N}_{\Delta}=\pi^{-1}(\Delta) \longrightarrow \mathcal{M}$ such that:

1. $g$ maps $\pi^{-1}(s)$ biholomorphically onto $\phi^{-1}(h(s))$ for each $s \in \Delta$ and

2. $g$ restricted to $\pi^{-1}\left(s_{0}\right)$ is the identity map on $M$.

Then the induced family $\left(h^{*} \mathcal{M}, D, h^{*} \phi\right)$ is exactly the restriction $\left(\mathcal{N}_{\Delta}, \Delta, \pi\right)$ of $(\mathcal{N}, D, \pi)$ to $\Delta$.

The proof of this lemma is obvious. First it is easy to verify (using the construction of (2.4.3.3)) that the induced family is biholomorphically equivalent to the restriction of the family on $D$ to $\Delta$. Then a suitable change of coordinates for the induced family, which identifies the biholomorphic map of $\left(h^{*} \phi\right)^{-1}\left(s_{0}\right)$ onto $\pi^{-1}\left(s_{0}\right)$ with the identity map of $M$, does the job.

Given a complex analytic family $(\mathcal{N}, D, \pi)$ with $s_{0} \in D$ such that $\pi^{-1}\left(s_{0}\right) \cong M_{t_{0}}$, Kodaira constructs the holomorphic maps $h, g$ (satisfying the conditions of the above lemma) first as formal power series by a method of solving infinitely many congruences using Čech cohomology a similar method is outlined in (3.4.5). He then proves that these formal power series indeed converge to give the desired holomorphic maps. He does this for any arbitrarily chosen complex analytic family $(\mathcal{N}, D, \pi)$ with $\pi^{-1}\left(s_{0}\right) \cong \phi^{-1}\left(t_{0}\right)$ and thus proves the theorem of completeness. The hypothesis that the infinitesimal Kodaira-Spencer map at the point $t_{0} \in B$ for the family $(\mathcal{M}, B, \phi)$ is surjective is used in the proof of the existence of formal power series for $g, h$ satisfying the desired properties.

\subsubsection{Effective Families and the Number of Moduli}

Let $(\mathcal{M}, B, \phi)$ be a complex analytic family of compact complex manifolds that is complete at $t_{0} \in B$. This is ensured, by the theorem of completeness of the previous subsection, if the infinitesimal Kodaira-Spencer map $\rho_{t_{0}}$ : $T_{t_{0}} B \longrightarrow \mathrm{H}^{1}\left(M_{t_{0}}, \Theta_{t_{0}}\right)$ is surjective. In this case we know that $(\mathcal{M}, B, \phi)$ contains all possible infinitesimal deformations of $M_{t_{0}}$. But it may happen that two distinct tangent directions at $t_{0}$ to $B$ may give rise to the same infinitesimal deformation of $M_{t_{0}}$. We would like to have a situation where this does not happen i.e., a situation in which $\rho_{t_{0}}$ is injective. Then $\rho_{t_{0}}$ would give an isomorphism of $T_{t_{0}} B$ with $\mathrm{H}^{1}\left(M_{t_{0}}, \Theta_{t_{0}}\right)$. More generally, we would like to have a situation where there are no "redundant directions". This naturally leads to the following. 
3.4.3.1 Definition. Let $(\mathcal{M}, B, \phi)$ be a complex analytic family of compact complex manifolds and $t_{0} \in B$. Then this family is said to be effective at $t_{0} \in B$ if the infinitesimal Kodaira-Spencer map $\rho_{t_{0}}: T_{t_{0}} B \longrightarrow$ $\mathrm{H}^{1}\left(M_{t_{0}}, \Theta_{t_{0}}\right)$ is injective. We say that $(\mathcal{M}, B, \phi)$ is effectively parametrized or that $t \in B$ is an effective parameter if $(\mathcal{M}, B, \phi)$ is effective at each $t \in B$.

Note that if $(\mathcal{M}, B, \phi)$ is effectively parametrized, then at each $t \in B$, distinct tangent directions give rise to distinct infinitesimal deformations of $M_{t}$ i.e., no two different tangent directions can define the same infinitesimal deformation of $M_{t}$ for each $t \in B$.

Now if $M$ is a compact complex manifold and $(\mathcal{M}, B, \phi)$ is a complex analytic family of compact complex manifolds with $t_{0} \in B$ such that $\phi^{-1}\left(t_{0}\right)=M$, then we would expect something special to happen if this family were both complete and effectively parametrized. This is justified by the following.

3.4.3.2 Theorem. Let $M$ be a compact complex manifold and $(\mathcal{M}, B, \phi)$ a complete effectively parametrized complex analytic family of compact complex manifolds with $t_{0} \in B$ such that $\phi^{-1}\left(t_{0}\right)=M$. Then we have the following:

1. Let $(\mathcal{N}, D, \pi)$ be any complete effectively parametrized complex analytic family of compact complex manifolds with $s_{0} \in D$ such that $\pi^{-1}\left(s_{0}\right)=M$. Then both $B$ and $D$ have the same dimension as manifolds.

2. Let $(\mathcal{N}, D, \pi)$ be a complex analytic family satisfying the hypotheses of (1) above. Then there exists a domain $E \subset D$ with $s_{0} \in E$ and an injective holomorphic map $h: E \longrightarrow B$ (i.e., an open immersion) mapping $s_{0}$ onto $t_{0}$ such that the family induced by $h$ is equivalent to the restriction of the family $\mathcal{N}$ to $E$. In this sense, an effectively parametrized complete complex analytic family with fiber $M$ is uniquely determined, provided it exists, locally at the point over which $M$ is the fiber.

Proof. (1): Since $\phi^{-1}\left(t_{0}\right)=M=\pi^{-1}\left(s_{0}\right)$, and since $(\mathcal{M}, B, \phi)$ is complete implies it is complete at $t_{0}$, there exists a domain $E \subset D$ with $s_{0} \in E$ and a holomorphic map $h: E \longrightarrow B$ with $h\left(s_{0}\right)=t_{0}$ such that $\left(h^{*} \mathcal{M}, E, h^{*} \phi\right)$ is equivalent to $\left(\mathcal{N}_{E}, E, \pi\right)$. Let the dimension of $E$ be $l$. If we choose $E$ sufficiently small so that it is covered by a single local complex coordinate system with coordinates $s=\left(s_{1}, \ldots, s_{l}\right)$, then we have that the tangent vectors $\left\langle\frac{\partial}{\partial s_{1}}, \ldots, \frac{\partial}{\partial s_{l}}\right\rangle$ form a basis over $\mathbb{C}$ of $T_{s} E=T_{s} D$. But since $(\mathcal{N}, D, \pi)$ is effectively parametrized, we see that so is its restriction $\left(\mathcal{N}_{E}, E, \pi\right)$. Hence 
$\rho_{s}: T_{s} E \longrightarrow \mathrm{H}^{1}\left(N_{s}, \Theta_{s}\right)$ is injective for each $s \in E$. Here, as usual, $N_{s}=\pi^{-1}(s)$. But since $\left(h^{*} \mathcal{M}, E, h^{*} \phi\right)$ is equivalent to $\left(\mathcal{N}_{E}, E, \pi\right)$, we have that $N_{s}$ is biholomorphic to $M_{h(s)}$ for each $s \in E$. Thus $\left\langle\frac{\partial N_{s}}{\partial s_{1}}, \ldots, \frac{\partial N_{s}}{\partial s_{l}}\right\rangle$ are linearly independent over $\mathbb{C}$ in $\mathrm{H}^{1}\left(N_{s}, \Theta_{s}\right)=\mathrm{H}^{1}\left(M_{h(s)}, \Theta_{h(s)}\right)$ for each $s \in E$.

Next let the dimension of $B$ be $m$ and $t=\left(t_{1}, \ldots, t_{m}\right)$ be a local complex coordinate in a sufficiently small neighborhood of $t_{0}=h\left(s_{0}\right) \in B$. Then choosing $E$ to be smaller if necessary, we have that $t=\left(t_{1}, \ldots, t_{m}\right)=h(s)$ and that $\left\langle\frac{\partial M_{t}}{\partial t_{1}}, \ldots, \frac{\partial M_{t}}{\partial t_{m}}\right\rangle$ are linearly independent over $\mathbb{C}$ in $\mathrm{H}^{1}\left(M_{h(s)}, \Theta_{h(s)}\right)$ $=\mathrm{H}^{1}\left(M_{t}, \Theta_{t}\right)$ for each $t$ in this local complex coordinate neighborhood, where we have used the fact that the image of the tangent space at $t$ to $B$ in $\mathrm{H}^{1}\left(M_{t}, \Theta_{t}\right)$ by $\rho_{t}$ has dimension $m$ because $(\mathcal{M}, B, \phi)$ is effectively parametrized. By theorem (3.3.1.1) we have the following commutative diagram:

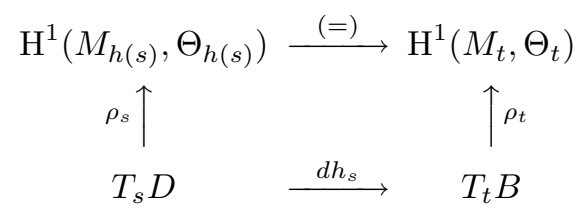

Further from the same theorem we also have by the chain rule:

$$
\rho_{s}\left(\frac{\partial}{\partial s_{\mu}}\right)=\frac{\partial N_{s}}{\partial s_{\mu}}=\sum_{\lambda=1}^{m} \frac{\partial t_{\lambda}}{\partial s_{\mu}} \frac{\partial M_{t}}{\partial t_{\lambda}},(1 \leq \mu \leq 1) .
$$

This shows that the image of $\rho_{s}$ is contained in the image of $\rho_{t}$ for each $s \in E$. Since $\left\langle\frac{\partial N_{s}}{\partial s_{1}}, \ldots, \frac{\partial N_{s}}{\partial s_{l}}\right\rangle$ are linearly independent over $\mathbb{C}$ as already noted, this forces $l \leq m$.

Now we carry out the above argument by reversing the roles of $(\mathcal{M}, B, \phi)$ and $(\mathcal{N}, D, \pi)$ to obtain $m \leq l$, whence $m=l$ as claimed.

Proof. (2): We have proved above that for each $s \in E, \rho_{s}\left(T_{s} D\right)=\rho_{t}\left(T_{t} B\right) \subset$ $\mathrm{H}^{1}\left(M_{t}, \Theta_{t}\right)$ where $t=h(s)$. Thus the determinant of the matrix

$$
\left(\frac{\partial t_{\lambda}}{\partial s_{\mu}}\right)
$$

is nonzero. But this matrix is precisely the Jacobian matrix of the holomorphic map $h: E \longrightarrow B$ (written in terms of the local coordinates at $s_{0} \in E$ and $\left.t_{0}=h\left(s_{0}\right) \in B\right)$. Hence $h$ has to be injective and thus $\Delta:=h(E)$ is a domain in $B$ containing $t_{0}$. Now take $E$ sufficiently small, so that $\Delta$ also becomes sufficiently small, in such a way that we may represent the deformation space of the restricted family $\left(\mathcal{M}_{\Delta}, \Delta, \phi\right)$ as

$$
\mathcal{M}_{\Delta}=\left(\cup_{j=1}^{k}\left(U_{j} \times \Delta\right)\right) / \sim_{\Delta}
$$


where $U_{1}, \ldots, U_{k}$ are the polydiscs which are glued via the transition functions $\left\{f_{j k}\left(z_{k}, t_{0}\right)\right\}$ to give the compact complex manifold $M_{t_{0}}$ and the equivalence relation $\sim_{\Delta}$ is defined thus: if $\left(z_{j}, t\right) \in U_{j} \times \Delta$ and $\left(z_{k}, t^{\prime}\right) \in U_{k} \times \Delta$ then $\left(z_{j}, t\right) \sim_{\Delta}\left(z_{k}, t^{\prime}\right)$ if any only if $t=t^{\prime}$ and $z_{j}=f_{j k}\left(z_{k}, t\right)$. Then we may write (cf. (3.3.1))

$$
\mathcal{N}_{E}=\left(\cup_{j=1}^{k}\left(U_{j} \times E\right)\right) / \sim_{E}
$$

where the equivalence relation $\sim_{E}$ is defined thus: if $\left(z_{j}, s\right) \in U_{j} \times E$ and $\left(z_{k}, s^{\prime}\right) \in U_{k} \times E$ then $\left(z_{j}, s\right) \sim_{E}\left(z_{k}, s^{\prime}\right)$ if and only if $s=s^{\prime}$ and $z_{j}=f_{j k}\left(z_{k}, h(s)\right)$. Now since $h: E \longrightarrow \Delta$ is biholomorphic, we consider $h$ as a coordinate transformation of one and the same parameter space (i.e., identify $E$ and $\Delta$ as biholomorphic complex structures on the same differentiable manifold). Then $\left(\mathcal{M}_{\Delta}, \Delta, \phi\right)$ and $\left(\mathcal{N}_{E}, E, \pi\right)$ can be considered as the same complex analytic family but with different choices of coordinate systems on $\mathcal{N}_{E}$ and $\mathcal{M}_{\Delta}$ QED

Motivated by the above theorem, we state the following.

3.4.3.3 Definition. Let $M$ be a compact complex manifold. Let $(\mathcal{M}, B, \phi)$ be a complete effectively parametrized complex analytic family with $t_{0} \in B$ such that $\phi^{-1}\left(t_{0}\right)=M$. We define the dimension of $B$ to be the number of moduli of $M$.

Again let us emphasize that this definition of number of moduli of a compact complex manifold does not depend on a particular choice of $(\mathcal{M}, B, \phi)$ because of the theorem just proved.

\subsubsection{Examples of Complete Effectively Parametrized Families: The Case of Complex Tori}

Recall the complex analytic families of complex tori denoted by $(\mathcal{B}, S, \phi)$ and $(\mathcal{C}, M, \psi)$ constructed respectively in (2.6.1) and (2.6.2). Recall that $(\mathcal{C}, M, \psi)$ is locally (over $M)$ equivalent to $(\mathcal{B}, S, \phi)$. We will show that $(\mathcal{B}, S, \phi)$ is a complete effectively parametrized family by showing that the infinitesimal Kodaira-Spencer map is an isomorphism at each $s \in S$, for then we may apply the theorem of completeness. Thus the number of moduli for any complex torus $T^{n}$ is defined and equals $n^{2}=\operatorname{dim}\left(\mathrm{H}^{1}\left(T^{n}, \Theta_{T^{n}}\right)\right)$. Notice that the dimension of $S$ is also $n^{2}$ by the very definition of $S$ (it can be identified naturally in an obvious manner with an open subset of $\mathbb{C}^{n^{2}}$ ).

Notice that the completeness and effectiveness of the family $\mathcal{B}$ imply that the family $(\mathcal{C}, M, \psi)$ is also complete and effectively parametrized, since it is locally equivalent to $(\mathcal{B}, S, \phi)$.

We have already mentioned in (2.6.2) that if $n \geq 2$, then given any nonempty open subset $U$ of $M$, there exists a point $t \in U$ such that the 
$\mathbf{S L}(2 n, \mathbb{Z})$-orbit of $t$ intersects $U$ in an infinite set of points: $\left\{t \delta_{1}, \ldots t \delta_{i}, \ldots\right\}$. These are infinitely many distinct points of $U$ over which the fibers of $\psi$ are all biholomorphic to each other. Hence we see that the effectivity and completeness of a family need not imply an injective parametrization in any neighborhood of any point of the parameter space.

Now we indicate a proof of the fact that the family $(\mathcal{B}, S, \phi)$ is complete and effectively parametrized. Let us write as usual:

$$
(\mathcal{B}, S, \phi)=\left\{B_{s}:=\phi^{-1}(s) ; s \in S\right\} .
$$

Further, as usual, let $\Theta_{s}$ denote the sheaf of germs of holomorphic vector fields on $B_{s}$. Let $p$ be the canonical locally biholomorphic covering map from $\mathbb{C}^{n} \times S \longrightarrow\left(\mathbb{C}^{n} \times S\right) / G=: \mathcal{B}$ (see (2.6.1) and (1.5.2.1.2))

Let $\left\{U_{i} ; i \in \mathbb{N}\right\}$ be a locally finite covering of $\mathcal{B}$ where each $U_{i}$ is chosen sufficiently small so that its inverse image under $p$ is a disjoint union of infinitely many domains $\left\{U_{i j} ; j \in \mathbb{N}\right\}$ of $\mathbb{C}^{n} \times S$ each of which is biholomorphically mapped by $p$ onto $U_{i}$. Choose one of the $U_{i j}$ for each $i$, say $U_{i 1}$. Let $\left(z^{1}, \ldots, z^{n}\right)$ be standard coordinates on $\mathbb{C}^{n}$ and $s=\left(s_{\beta}^{\alpha}\right) \in S \subset \mathbb{C}^{n^{2}}$ be coordinates on $S$ induced (from the standard coordinates on $\mathbb{C}^{n^{2}}$ ) via its inclusion as an open subset of $\mathbb{C}^{n^{2}}$ as explained in (2.6.1). Thus we get $\left(z^{1}, \ldots, z^{n}, s\right)$ as coordinates on $\mathbb{C}^{n} \times S$. Let $\left(z_{i}^{1}, \ldots, z_{i}^{n}, s\right)$ be the restriction of these coordinates on $\mathbb{C}^{n} \times S$ to $U_{i 1}$ for each $i$. Since $p$ : $U_{i 1} \longrightarrow U_{i}$ is biholomorphic, we may consider, for each $i$, the coordinates $\left(z_{i}^{1}, \ldots z_{i}^{n}, s\right)$ on $U_{i 1}$ as coordinates on $U_{i}$ via $p$. We write for simplicity: $\left(z_{i}^{1}, \ldots, z_{i}^{n}, s\right)=\left(z_{i}, s\right)$. Hence we get the local complex coordinate system $\left\{\left(U_{i},\left(z_{i}, s\right)\right) ; i \in \mathbb{N}\right\}$ on $\mathcal{B}$.

By the definition of $\mathcal{B}$ we have on $U_{i} \cap U_{k} \neq \emptyset$ :

$$
z_{i}^{\alpha}=z_{k}^{\alpha}+m_{i k}^{\alpha}+\sum_{\beta=1}^{n} m_{i k}^{n+\beta} s_{\beta}^{\alpha}, m_{i k}^{j} \in \mathbb{Z},(1 \leq \alpha \leq n) .
$$

Therefore the transition functions of $\mathcal{B}$ relative to the covering $\left\{U_{i} ; i \in \mathbb{N}\right\}$ are given by:

$$
f_{i k}^{\alpha}\left(z_{k}, s\right)=z_{k}^{\alpha}+m_{i k}^{\alpha}+\sum_{\beta=1}^{n} m_{i k}^{n+\beta} s_{\beta}^{\alpha}, m_{i k}^{j} \in \mathbb{Z},(1 \leq \alpha \leq n) .
$$

Hence the infinitesimal deformation of $B_{s}$ along $\left(\partial / \partial s_{\beta_{0}}^{\alpha_{0}}\right)$ is given by the cohomology class $\theta(s) \in \mathrm{H}^{1}\left(B_{s}, \Theta_{s}\right)$ defined by the 1 -cocycle $\left\{\theta_{i k}(s)\right\}$ where:

$$
\theta_{i k}(s)=\sum_{\gamma=1}^{n} \frac{\partial f_{i k}^{\gamma}\left(z_{k}, s\right)}{\partial s_{\beta_{0}}^{\alpha_{0}}} \frac{\partial}{\partial z_{i}^{\gamma}}=m_{i k}^{n+\beta_{0}} \frac{\partial}{\partial z_{i}^{\alpha_{0}}} .
$$


Let $\Psi_{s}$ be the sheaf of germs of $C^{\infty}$ vector fields of the form $\sum_{\alpha} \psi^{\alpha} \frac{\partial}{\partial z^{\alpha}}$ over $B_{s}$. We have the obvious short exact sequence of sheaves on $B_{s}$ given by

$$
0 \longrightarrow \Theta_{s} \stackrel{i}{\longrightarrow} \Psi_{s} \stackrel{\bar{\partial}}{\longrightarrow} \bar{\partial} \Psi_{s} \longrightarrow 0
$$

from which we obtain a long exact sequence in cohomology (see (A.8)):

$$
\begin{aligned}
0 \longrightarrow \mathrm{H}^{0}\left(B_{s}, \Theta_{s}\right) & \stackrel{i}{\longrightarrow} \mathrm{H}^{0}\left(B_{s}, \Psi_{s}\right) \stackrel{\bar{\partial}}{\longrightarrow} \mathrm{H}^{0}\left(B_{s}, \bar{\partial} \Psi_{s}\right) \stackrel{\delta^{*}}{\longrightarrow} \mathrm{H}^{1}\left(B_{s}, \Theta_{s}\right) \longrightarrow \\
\longrightarrow & \mathrm{H}^{1}\left(B_{s}, \Psi_{s}\right) \longrightarrow \mathrm{H}^{1}\left(B_{s}, \bar{\partial} \Psi_{s}\right) \longrightarrow \cdots
\end{aligned}
$$

It can be checked that the map $\mathrm{H}^{1}\left(B_{s}, \Psi_{s}\right) \longrightarrow \mathrm{H}^{1}\left(B_{s}, \bar{\partial} \Psi_{s}\right)$ of the above long exact sequence is injective. Hence $\delta^{*}$ is surjective. Hence we have:

$$
\mathrm{H}^{1}\left(B_{s}, \Theta_{s}\right)=\mathrm{H}^{0}\left(B_{s}, \bar{\partial} \Psi_{s}\right) / \bar{\partial} \mathrm{H}^{0}\left(B_{s}, \Psi_{s}\right) .
$$

We shall use Čech cohomology for the calculations below (see the remarks at the end of (A.9)). First of all, from the relations (3.5) above we get:

$$
\sum_{\beta=1}^{n} m_{i k}^{n+\beta}\left(s_{\beta}^{\alpha}-\overline{s_{\beta}^{\alpha}}\right)=z_{i}^{\alpha}-\overline{z_{i}^{\alpha}}-\left(z_{k}^{\alpha}-\overline{z_{k}^{\alpha}}\right) .
$$

By the definition of $S$, since $s=\left(s_{\beta}^{\alpha}\right) \in S$, the determinant of $(s-\bar{s})$ is nonzero; hence we can set $u=(s-\bar{s})^{-1}=\left(u_{\beta}^{\alpha}\right)$. Then we get

$$
m_{i k}^{n+\beta_{0}}=\sum_{\gamma=1}^{n}\left(\overline{z_{k}^{\gamma}}-z_{k}^{\gamma}\right) u_{\gamma}^{\beta_{0}}-\sum_{\gamma=1}^{n}\left(\overline{z_{i}^{\gamma}}-z_{i}^{\gamma}\right) u_{\gamma}^{\beta_{0}} .
$$

Now set on each nonempty intersection $U_{k} \cap B_{s}$

$$
\psi_{k}=\sum_{\gamma=1}^{n}\left(\overline{z_{k}^{\gamma}}-z_{k}^{\gamma}\right) u_{\gamma}^{\beta_{0}} \frac{\partial}{\partial z_{k}^{\alpha_{0}}} .
$$

Then from relations (3.6) and (3.8) we get on each nonempty intersection $U_{i} \cap U_{k} \cap B_{s}$

$$
\psi_{k}-\psi_{i}=\sum_{\gamma=1}^{n}\left(\left(\overline{z_{k}^{\gamma}}-z_{k}^{\gamma}\right)-\left(\overline{z_{i}^{\gamma}}-z_{i}^{\gamma}\right)\right) u_{\gamma}^{\beta_{0}} \frac{\partial}{\partial z_{i}^{\alpha_{0}}}=m_{i k}^{n+\beta_{0}} \frac{\partial}{\partial z_{i}^{\alpha_{0}}}=\theta_{i k}(s),
$$

where we have used the fact that $\frac{\partial}{\partial z_{i}^{\alpha_{0}}}=\frac{\partial}{\partial z_{k}^{\alpha_{0}}}$ on $U_{i} \cap U_{k}$. Let $\mathcal{U}_{s}=$ $\left\{U_{k s} ; U_{k s}:=U_{k} \cap B_{s} \neq \emptyset\right\}$. Note that $\psi_{k}$ is a section of $\Psi_{s}$ over $U_{k s}$ and 
$\bar{\partial} \psi_{k}-\bar{\partial} \psi_{i}=0$ on $U_{k s} \cap U_{i s} \neq \emptyset$. Hence these $\bar{\partial} \psi_{k}$ patch up to give a global section $\varphi(s)$ of $\bar{\partial} \Psi_{s}$ i.e., a section over $B_{s}$ such that

$$
\left.\varphi(s)\right|_{U_{k s}}=\bar{\partial} \psi_{k}=\sum_{\gamma=1}^{n} u_{\gamma}^{\beta_{0}}\left(d \overline{z_{k}^{\gamma}} \otimes \frac{\partial}{\partial z_{k}^{\alpha_{0}}}\right) .
$$

Now let $c^{0}=\left\{\psi_{k}\right\} \in C^{0}\left(\mathcal{U}_{s}, \Psi_{s}\right)$. Then $\bar{\partial} c^{0}=\varphi(s)$ and $\delta c^{0}:=\left\{\psi_{k}-\psi_{i}\right\}=$ $\left\{\theta_{i k}(s)\right\}$ (see (A.9)). By analyzing the way in which $\delta^{*}$ is defined, it is easy to see that these last two calculations imply that $\delta^{*} \varphi(s)=\theta(s)$ where $\theta(s) \in \mathrm{H}^{1}\left(B_{s}, \Theta_{s}\right)$ is the cohomology class determined by $\left\{\theta_{i k}(s)\right\}$. Now let $\mathbb{H}^{0,1}\left(T B_{s}\right)$ denote harmonic vector $T B_{s}$-forms of type $(0,1)$ where $T B_{s}$ denotes the tangent bundle of $B_{s}$. Then it can be checked that under the Hodge-Dolbeault isomorphism we have:

$$
\mathrm{H}^{1}\left(B_{s}, \Theta_{s}\right) \cong \mathrm{H}_{\bar{\partial}}^{0,1}\left(B_{s}, T B_{s}\right) \cong \mathbb{H}^{0,1}\left(T B_{s}\right): \theta(s) \mapsto \varphi(s) .
$$

Also, since it turns out by explicit calculations that

$$
\mathbb{H}^{0,1}\left(T B_{s}\right)=\left\{\varphi ; \varphi=\sum_{\alpha=1}^{n} \sum_{\beta=1}^{n} c_{\beta}^{\alpha}\left(\overline{d z^{\beta}} \otimes \frac{\partial}{\partial z^{\alpha}}\right), c_{\beta}^{\alpha} \in \mathbb{C}\right\},
$$

we have the dimension of $\mathrm{H}^{1}\left(B_{s}, \Theta_{s}\right)$ as $n^{2}$. Further, since the determinant of $u=(s-\bar{s})^{-1}$ is nonzero, the elements

$$
\left\langle\varphi(s)=\sum_{\gamma=1}^{n} u_{\gamma}^{\beta_{0}}\left(d \overline{z^{\gamma}} \otimes \frac{\partial}{\partial z^{\alpha_{0}}}\right) ; 1 \leq \alpha_{0}, \beta_{0} \leq n\right\rangle
$$

are linearly independent. Hence the infinitesimal Kodaira-Spencer map $\rho_{s}: T_{s} S \longrightarrow \mathrm{H}^{1}\left(B_{s}, \Theta_{s}\right)$ is an isomorphism. Hence we have proved the following.

3.4.4.1 Theorem. The complex analytic families $(\mathcal{B}, S, \phi)$ and $(\mathcal{C}, M, \psi)$ are complete and effectively parametrized. The number of moduli of any complex $n$-dimensional torus $B$ is defined and equals $n^{2}$. We also have that the dimension of $H^{1}(B, \Theta)$ equals the number of moduli of $B$.

3.4.4.2 Remark. As mentioned in (2.7.1), a complex $n$-dimensional torus $T^{n}$ is algebraizable if and only if its period matrix is a Riemann matrix. In this case $T^{n}$ can be embedded as a closed submanifold of some complex projective space $P_{\mathbb{C}}^{m}$. Assume $n \geq 3$. By Bertini's Theorem (cf. Hartshorne's book: Algebraic Geometry, chap. II, sec. 8, cited under the References at the end), for a sufficiently general hyperplane $H$ of $P_{\mathbb{C}}^{m}$, the intersection $M:=T^{n} \cap H$ is a nonsingular hypersurface in $T^{n}$. 
Kodaira and Spencer prove in [6] that given a complex analytic family $\left\{M_{t} ; t \in B \subset \mathbb{C}^{m}\right\}$ with $M_{0}=M,(0 \in B)$, there exists a complex analytic family $\left\{T_{t}^{n} ; t \in \Delta \subset B, 0 \in \Delta\right\}$ of $n$-dimensional complex tori with $T_{0}^{n}=T^{n}$ (for $\Delta$ a sufficiently small polydisc) such that $M_{t} \subset T_{t}^{n}$ for $t \in \Delta$ which implies $T_{t}^{n}$ is algebraic for each $t \in \Delta$. Hence $\left\{T_{t}^{n} ; t \in \Delta \subset B\right\}$ consists purely of algebraic deformations.

Kodaira and Spencer construct a complete effectively parametrized complex analytic family of algebraic $n$-dimensional tori and thus prove that the number of (algebraic) moduli of any algebraic torus equals $n(n+1) / 2$. However, we have just seen that the number of moduli of a complex $n$ dimensional torus (whether algebraic or not) equals $n^{2}$. The increase in the number of moduli in this case is obviously because we allowed non-algebraic deformations also. Note that if we allow only algebraic deformations, we are imposing the additional condition on the deformations (tori) that their period matrices should be Riemann matrices. This condition reduces the number of effective parameters from $n^{2}$ to $n(n+1) / 2$.

\subsubsection{Obstructions to Infinitesimal Deformation of Complex Structure: A Reformulation}

In this subsection, we continue with the study of obstruction theory begun in (3.4.1). Recall from (3.4.1) that if $M$ is a compact complex manifold and $\theta \in \mathrm{H}^{1}(M, \Theta)$ such that $[\theta, \theta](\neq 0) \in \mathrm{H}^{2}(M, \Theta)$, then $[\theta, \theta]$ is called a primary obstruction to infinitesimal deformation of complex structure of $M$. In fact, if $\theta$ were to correspond to some infinitesimal deformation of $M$, then necessarily we have $[\theta, \theta]=0$ (cf. theorem (3.4.1.1)).

We formulate below the notions of secondary obstructions, tertiary obstructions, and so on. These are seen to vanish if $\mathrm{H}^{2}(M, \Theta)=0$. It then seems natural to expect that in situations where $\mathrm{H}^{2}(M, \Theta)=0$, something good might happen. This is indeed true and is expressed by the Theorem of Existence described in the next subsection. But for now, we see how higher order obstructions are defined and in the process reformulate the notion of primary obstruction.

We will formulate the higher order obstructions by investigating the following situation. Let $\theta \in \mathrm{H}^{1}(M, \Theta)$ be given and let us assume that there exists a complex analytic family $(\mathcal{M}, \mathbb{C}, \phi)$ with $\phi^{-1}(0)=M$ and with the infinitesimal deformation of $M$ at $0 \in \mathbb{C}$ along the direction prescribed by the tangent vector $\frac{d}{d t}$ given precisely by $\theta$.

If we choose $\Delta=\{t \in \mathbb{C} ;|t|<r\}$ to be a sufficiently small disc, then we can write the restriction of $\mathcal{M}$ to $\Delta$ as follows (cf. (2.4.4)):

$$
\mathcal{M}_{\Delta}=\left(U_{j=1}^{l}\left(U_{j} \times \Delta\right)\right) / \sim_{\Delta}
$$


where each $U_{j}$ is a polydisc in $\mathbb{C}^{n}$ with $z_{j}=\left(z_{j}^{1}, \ldots, z_{j}^{n}\right)$ as complex coordinates (where $n$ is the dimension of $M$ ) and $\mathcal{M}_{\Delta}$ is the complex manifold obtained by glueing the domains $\left\{U_{j} \times \Delta ; j=1, \ldots, l\right\}$ via the transition functions $\left\{f_{j k}\left(z_{k}, t\right)\right\}$ i.e., the glueing is determined by identifying points equivalent under $\sim_{\Delta}$ where $\left(z_{j}, t\right) \in U_{j} \times \Delta$ and $\left(z_{k}, t^{\prime}\right) \in U_{k} \times \Delta$ are equivalent under $\sim_{\Delta}$ if and only if $t=t^{\prime}$ and $z_{j}=f_{j k}\left(z_{k}, t\right)$. Each transition function

$$
f_{j k}\left(z_{k}, t\right)=\left(f_{j k}^{1}\left(z_{k}, t\right), \ldots, f_{j k}^{n}\left(z_{k}, t\right)\right)
$$

is a holomorphic function on the nonempty intersection $\left(U_{k} \times \Delta\right) \cap\left(U_{j} \times \Delta\right) \subset$ $U_{k} \times \Delta$. Further these transition functions satisfy the following patching conditions:

$$
\begin{aligned}
& \left(U_{i} \times \Delta\right) \cap\left(U_{j} \times \Delta\right) \cap\left(U_{k} \times \Delta\right) \neq \emptyset \\
& \quad \Rightarrow f_{i k}^{\alpha}\left(z_{k}, t\right)=f_{i j}^{\alpha}\left(f_{j k}\left(z_{k}, t\right), t\right),(1 \leq \alpha \leq n) .
\end{aligned}
$$

Now $M=\phi^{-1}(0)$, so if we identify $U_{j} \times\{0\}$ with $U_{j}$, then $\mathcal{U}:=\left\{U_{j} ; j=\right.$ $1, \ldots, l\}$ is a finite open covering for $M$ with $z_{j}=\left(z_{j}^{1}, \ldots, z_{j}^{n}\right)$ as local complex coordinates on $U_{j}$.

Let us expand each component of each transition function as a power series in $t$ :

$$
f_{j k}^{\alpha}\left(z_{k}, t\right)=\sum_{\nu=0}^{\infty} f_{j k, \nu}^{\alpha}\left(z_{k}\right) t^{\nu} .
$$

Since the transition functions for $M$ relative to $\mathcal{U}$ are given by $\left\{f_{j k}\left(z_{k}, 0\right)\right\}$, we see that $\left\{f_{j k, 0}\left(z_{k}\right)\right\}$ are precisely these transition functions. Since $M$ is already given, we may assume that these transition functions are also already given alongwith $\mathcal{U}$.

Since $\theta \in \mathrm{H}^{1}(M, \Theta)$ has been assumed to be the infinitesimal deformation of $M$ along $\frac{d}{d t}$, we have by the very definition of infinitesimal deformation (see (3.2.8)) that $\theta$ is represented by the element $\left\{\theta_{j k}\right\} \in Z^{1}(\mathcal{U}, \Theta)$ where

$$
\theta_{j k}=\sum_{\alpha=1}^{n} \theta_{j k}^{\alpha}\left(z_{j}\right) \frac{\partial}{\partial z_{j}^{\alpha}},
$$

and where

$$
\theta_{j k}^{\alpha}\left(z_{j}\right)=f_{j k, 1}^{\alpha}\left(z_{k}\right), z_{k}=f_{k j, 0}\left(z_{j}\right) .
$$

Hence, starting with a given compact complex manifold $M$, a finite system of local complex coordinates $\mathcal{U}=\left\{\left(U_{j}, z_{j}\right) ; 1 \leq j \leq l\right\}$ for $M$ where $U_{j} \subset \mathbb{C}^{n}$ is a polydisc and $z_{j}$ is the coordinate on $U_{j}$ for each $j$, transition functions $\left\{f_{j k, 0}\left(z_{k}\right)\right\}$ relative to this cover, a cohomology class $\theta \in \mathbf{H}^{1}(M, \Theta)$ and a representative $\left\{\theta_{j k}\right\} \in$ $Z^{1}(\mathcal{U}, \Theta)$ of $\theta$ such that $(3.11)$ above holds, in order that $\theta$ may 
correspond to an infintesimal deformation of $M$, it is sufficient if we could construct the holomorphic functions $f_{j k}\left(z_{k}, t\right)$ on the domains $\left(U_{k} \times \Delta\right) \cap\left(U_{j} \times \Delta\right) \subset U_{k} \times \Delta$ (for some sufficiently small disc $\Delta \subset \mathbb{C}$ containing the origin) such that they satisfy (3.9), (3.10) and (3.12). For in such a case, $\left(\mathcal{M}_{\Delta}, \Delta, \phi\right):=\left\{M_{t}:=\phi^{-1}(t) ; t \in \Delta\right\}$ is a complex analytic family defined by

$$
\mathcal{M}_{\Delta}=\left(\cup_{j=1}^{l}\left(U_{j} \times \Delta\right)\right) / \sim_{\Delta}
$$

where $\sim_{\Delta}$ is an equivalence relation (as described earlier) and further for this family the infintesimal deformation at $t=0$ along the tangent vector $\frac{d}{d t}$ is precisely given by $\theta$.

Now in view of $(3.10)$, it is even enough to find the coefficients $f_{j k, \nu}^{\alpha}\left(z_{k}\right)$ there (for each $\nu=2,3, \ldots$ ) as holomorphic functions and then prove that the expression on the right-hand-side of (3.10) converges for $t \in \Delta$ (for $\Delta$ sufficiently small) and further that (3.9) and (3.12) are also satisfied.

Before we continue further, we introduce an extension of the notion of Cech cohomology (see (A.9)) with respect to the cover $\mathcal{U}$ on $M$ as follows.

Let $\mathcal{F}$ be any sheaf of abelian groups on $M$ (see (A.1)). An extended 1 -cochain on $\mathcal{U}$ with values in $\mathcal{F}$ is a set $\left\{\sigma_{j k}\right\}$ where for each nonempty intersection $U_{j} \cap U_{k}, \sigma_{j k}$ is a section of $\mathcal{F}$ over this intersection and $\sigma_{j j}=0$ for each $j$.

An extended 2-cocycle on $\mathcal{U}$ with values in $\mathcal{F}$ is a set $\left\{\sigma_{i j k}\right\}$ where for each nonempty intersection $U_{i} \cap U_{j} \cap U_{k}, \sigma_{i j k}$ is a section of $\mathcal{F}$ over this intersection satisfying the conditions $\sigma_{i i k}=\sigma_{i k k}=0$ for each $i, k$ and further on each nonempty intersection $U_{h} \cap U_{i} \cap U_{j} \cap U_{k}$, we have:

$$
\sigma_{i j k}-\sigma_{h j k}+\sigma_{h i k}-\sigma_{h i j}=0 .
$$

For any extended 1-cochain $\left\{\sigma_{i k}\right\}$ we define its coboundary as follows:

$$
\delta\left\{\sigma_{i k}\right\}=\left\{\tau_{i j k}\right\}, \tau_{i j k}:=\sigma_{j k}-\sigma_{i k}+\sigma_{i j} .
$$

Then it is verified that $\delta\left\{\sigma_{i k}\right\}=\left\{\tau_{i j k}\right\}$ is an extended 2-cocycle. Let $\widehat{C}^{1}(\mathcal{U}, \mathcal{F})$ be the abelian group of all extended 1 -cochains and let $\widehat{Z}^{2}(\mathcal{U}, \mathcal{F})$ be the abelian group of all extended 2-cocylces. Now we define the extended 2nd cohomology group relative to $\mathcal{U}$ with values in $\mathcal{F}$ to be the quotient:

$$
\widehat{\mathrm{H}}^{2}(\mathcal{U}, \mathcal{F})=\widehat{Z}^{2}(\mathcal{U}, \mathcal{F}) / \delta \widehat{C}^{1}(\mathcal{U}, \mathcal{F}) .
$$

Notice that if we compare the above definitions with the usual definitions (A.9) of Čech cohomology relative to $\mathcal{U}$ with values in $\mathcal{F}$, then we have:

$$
\begin{gathered}
Z^{2}(\mathcal{U}, \mathcal{F}) \subset \widehat{Z}^{2}(\mathcal{U}, \mathcal{F}), C^{1}(\mathcal{U}, \mathcal{F}) \subset \widehat{C}^{1}(\mathcal{U}, \mathcal{F}) \\
Z^{2}(\mathcal{U}, \mathcal{F}) \cap \delta \widehat{C}^{1}(\mathcal{U}, \mathcal{F})=\delta C^{1}(\mathcal{U}, \mathcal{F}),
\end{gathered}
$$


and hence we have the inclusion of abelian groups

$$
\mathrm{H}^{2}(\mathcal{U}, \mathcal{F}) \hookrightarrow \widehat{\mathrm{H}}^{2}(\mathcal{U}, \mathcal{F})
$$

It can be verified using a partition of unity subordinate to $\mathcal{U}$ and Dolbeault's Lemma that if $\mathrm{H}^{2}(M, \Theta)=0$ then $\widehat{\mathrm{H}}^{2}(\mathcal{U}, \Theta)=0$ where $\Theta$ as usual is the sheaf of germs of holomorphic vector fields on $M$.

Now we resume the discussion preceding the above (digression of) definitions. Given any formal power series in $t$ of the form $f(t)=\Sigma_{\nu=0}^{\infty} f_{\nu} t^{\nu}$, we denote its $n$-th partial sum by $S^{n}(f(t))$ :

$$
S^{n}(f(t)):=\sum_{\nu=0}^{n} f_{\nu} t^{\nu}
$$

So suppose we start with arbitrary power series $\left\{f_{j k}\left(z_{k}, t\right)\right\}$ given by (3.10) and satisfying (3.12). We want these power series to be modified such that they satisfy (3.9), for in such a case, $\theta$ does correspond to an infinitesimal deformation of $M$ as noted earlier. From (3.9) we get the following infinitely many congruences, one for each positive integer $\nu$, all of which put together are equivalent to (3.9):

$$
\begin{aligned}
\mathrm{P}(\nu): E(\alpha, i, j, k, \nu):= & S^{\nu}\left(f_{i k}^{\alpha}\left(z_{k}, t\right)\right) \\
& -S^{\nu}\left(f_{i j}^{\alpha}\left(S^{\nu}\left(f_{j k}^{1}\left(z_{k}, t\right)\right), \ldots, S^{\nu}\left(f_{j k}^{n}\left(z_{k}, t\right)\right), t\right)\right) \\
= & 0 \bmod \left(t^{\nu}\right),(1 \leq \alpha \leq n) .
\end{aligned}
$$

Clearly $\mathrm{P}(1)$ holds, for this can be verified using the assumed relations (3.12).

Let $\Gamma_{i j k, 2}^{\alpha}\left(z_{k}\right)$ be the coefficient of $t^{2}$ in $E(\alpha, i, j, k, 2)$. Then $\Gamma_{i j k, 2}^{\alpha}\left(z_{k}\right)$ is a holomorphic function on $U_{i} \cap U_{j} \cap U_{k}(\neq \emptyset) \subset M$. Now it can be verified that $\mathrm{P}(2)$ holds if and only if the functions $f_{j k, 2}^{\alpha}$ satisfy the following conditions:

$\sum_{\alpha} \Gamma_{i j k, 2}^{\alpha}\left(z_{k}\right) \frac{\partial}{\partial z_{i}^{\alpha}}=\sum_{\beta} f_{j k, 2}^{\beta}\left(z_{k}\right) \frac{\partial}{\partial z_{j}^{\beta}}-\sum_{\alpha} f_{i k, 2}^{\alpha}\left(z_{k}\right) \frac{\partial}{\partial z_{i}^{\alpha}}+\sum_{\alpha} f_{i j, 2}^{\alpha}\left(z_{j}\right) \frac{\partial}{\partial z_{i}^{\alpha}}$.

Now set

$$
\Gamma_{i j k, 2}=\sum_{\alpha} \Gamma_{i j k, 2}^{\alpha}\left(z_{k}\right) \frac{\partial}{\partial z_{i}^{\alpha}}, \sigma_{j k, 2}=\sum_{\beta} f_{j k, 2}^{\beta}\left(z_{k}\right) \frac{\partial}{\partial z_{j}^{\beta}} .
$$

In the above equations, $z_{k}, z_{j}=f_{j k, 0}\left(z_{k}\right)$ and $z_{i}=f_{i j, 0}\left(z_{j}\right)=f_{i k, 0}\left(z_{k}\right)$ are local coordinates of one and the same point of $M$ in $U_{i} \cap U_{j} \cap U_{k} \neq \emptyset$. 
Thus $\mathrm{P}(2)$ holds if and only if $\delta\left\{\sigma_{j k, 2}\right\}=\left\{\Gamma_{i j k, 2}\right\}$. In other words, if $\mathrm{P}(2)$ holds then the element $\Gamma_{2} \in \widehat{\mathrm{H}}^{2}(\mathcal{U}, \Theta)$ defined by the extended 2-cocycle $\left\{\Gamma_{i j k, 2}\right\}$ is zero, Further, a calculation shows that $\Gamma_{2}=\frac{1}{2}[\theta, \theta]$, where $[\theta, \theta]$ is considered as an element of the extended 2 nd cohomology group relative to the open cover $\mathcal{U}$ with values in $\Theta$ via the inclusion $\mathrm{H}^{2}(\mathcal{U}, \Theta) \hookrightarrow \widehat{\mathrm{H}}^{2}(\mathcal{U}, \Theta)$. Hence we see that if there exist holomorphic functions $\left\{f_{j k, 2}\right\}$ such that $\mathrm{P}(2)$ were to hold then the obstruction $[\theta, \theta]$ must vanish. This is the essence of theorem (3.4.1.1).

Now conversely suppose that $\Gamma_{2}=\frac{1}{2}[\theta, \theta]=0$. Hence there exists an extended 1-cochain $\left\{\sigma_{j k}\right\} \in \widehat{C}^{1}(\mathcal{U}, \Theta)$ with $\delta\left\{\sigma_{j k}\right\}=\left\{\Gamma_{i j k, 2}\right\}$. Modify the power series $\left\{f_{j k}\left(z_{k}, t\right)\right\}$ by putting $f_{j k, 2}$ to be the coefficient functions of $\sigma_{j k}$. Then it is easily verified that $\mathrm{P}(2)$ holds. Thus when $\Gamma_{2}=0$, we are able to get $\left\{f_{j k, 2}^{\alpha}\left(z_{k}\right)\right\}$ such that $\mathrm{P}(2)$ holds.

Again let $\Gamma_{2}=0$ and define $\Gamma_{i j k, 3}^{\alpha}$ to be the coefficient of $t^{3}$ in $E(\alpha, i, j, k, 3)$. We can as before show that if $\Gamma_{3}$, the extended 2nd cohomology class defined by the extended 2-cocycle $\left\{\Gamma_{i j k, 3}\right\}$, vanishes, then there exist holomorphic functions $\left\{f_{j k, 3}\right\}$ such that $\mathrm{P}(3)$ holds.

Inductively, we can prove that if $\Gamma_{2}=\Gamma_{3}=\cdots=\Gamma_{\nu}=0$, then we may define $\Gamma_{\nu+1}$ and that if this element vanishes, then there exist holomorphic functions $\left\{f_{j k, \nu+1}\right\}$ such that $\mathrm{P}(\nu+1)$ holds.

Hence we see that $\Gamma_{i} \in \widehat{\mathrm{H}}^{2}(\mathcal{U}, \Theta), i=2,3, \ldots$ are indeed obstructions to infinitesimal deformation of $M$, if they do not vanish. We thus call $\Gamma_{\nu+1}$ the $\nu$-th obstruction. Hence $\Gamma_{2}=\frac{1}{2}[\theta, \theta]$ is called the primary obstruction associated to $\theta$, provided it does not vanish. Note that $\Gamma_{\nu+1}$ is not considered to be defined unless all lower order obstructions are already zero i.e., unless we have $\Gamma_{2}=\cdots=\Gamma_{\nu}=0$. Moreover, note that $\Gamma_{\nu+1}$ depends on the choice of $\mathcal{U}$; further, for each choice of $\mathcal{U}, \Gamma_{\nu+1}$ depends on the choice of $\left\{f_{j k, \nu}\right\}$ with $\delta\left\{f_{j k, \nu}\right\}=\left\{\Gamma_{i j k, \nu}\right\}$.

However, if $\mathrm{H}^{2}(M, \Theta)=0$, then because $\widehat{\mathrm{H}}^{2}(\mathcal{U}, \Theta)=0$ also, we see that all obstructions $\left\{\Gamma_{\nu} ; \nu \geq 2\right\}$ corresponding to any element $\theta \in \mathrm{H}^{1}(M, \Theta)$ and relative to any cover $\mathcal{U}$ vanish. Hence, if $\mathrm{H}^{2}(M, \Theta)=0$, we are atleast able to get formal power series of the form $\left\{f_{j k}\left(z_{k}, t\right)\right\}$ satisfying (3.9), (3.10) and (3.12). Of course, even then, (3.10) needs to be proved to be convergent. Nevertheless, the preceeding formulation of obstructions shows that the condition $\mathrm{H}^{2}(M, \Theta)=0$ can be expected to be a reasonably good condition. This is how Kodaira and Spencer were inspired to take $\mathrm{H}^{2}(M, \Theta)=0$ as the right hypothesis under which they could expect a general theorem on existence of infinitesimal deformations to be true. This is justified by their Theorem of Existence described in the next subsection and also by Kuranishi's more general existence theorem described in (3.6.3). 
Moreover, the above method of constructing formal power series by solving congruences using Čech cohomology has been used by Kodaira-Spencer to prove their Theorems of Existence and Completeness.

\subsubsection{The Theorem of Existence and Number of Moduli}

The following fundamental theorem of Kodaira and Spencer asserts that the hypothesis of vanishing of $\mathrm{H}^{2}(M, \Theta)$ for a compact complex manifold $M$ is indeed sufficient to ensure that every element of $\mathrm{H}^{1}(M, \Theta)$ corresponds to some infinitesimal deformation of $M$. We have already explained in detail in the previous subsection how this hypothesis arises in a natural way. The following theorem also gives necessary and sufficient conditions under which the number of moduli of $M$ is defined under such a hypothesis.

3.4.6.1 Theorem of Existence. Let $M$ be a compact complex manifold such that $H^{2}(M, \Theta)=0$. Then we have the following.

1. There exists a complex analytic family $(\mathcal{M}, B, \phi)=\left\{M_{t}:=\phi^{-1}(t) ; t \in\right.$ $B\}$ with $0 \in B \subset \mathbb{C}^{m}$ such that $\phi^{-1}(0)=M$ and the infinitesimal Kodaira-Spencer map $\rho_{0}: T_{0} B \longrightarrow H^{1}(M, \Theta)$ is an isomorphism. Thus $(\mathcal{M}, B, \phi)$ is both complete and effective at $0 \in B$.

2. The following are equivalent:

(a) The number of moduli is defined for $M$.

(b) The restriction of the family $(\mathcal{M}, B, \phi)$ of (1) above to a polydisc $\Delta$ such that $0 \in \Delta \subset B$ is regular for $\Delta$ sufficiently small.

If (either of) the above hold, then the restricted family $\left(\mathcal{M}_{\Delta}, \Delta, \phi\right)$ is a complete effectively parametrized complex analytic family for $\Delta$ sufficiently small and hence the number of moduli of $M$ is $m$; further, this number of moduli is also equal to the dimension of $H^{1}(M, \Theta)$. Moreover, $\rho_{t}$ for $t \in \Delta$ is an isomorphism for $\Delta$ sufficiently small.

3. If $H^{0}(M, \Theta)=0$, then (b) of (2) above holds and (hence) the number of moduli of $M$ is defined and equals the dimension of $H^{1}(M, \Theta)$.

Proof. For the proof of (1), refer to Kodaira's book [5], sec. 5.3.

The proof of the implication " $(a) \Rightarrow(b)$ " of (2) is as follows: Let $\mu$ be the number of moduli of $M$. Hence there exists a complete effectively parametrized complex analytic family $(\mathcal{N}, D, \pi), 0 \in D \subset \mathbb{C}^{\mu}, \pi^{-1}(0)=M$. 
Since $(\mathcal{N}, D, \pi)$ is complete at $0 \in D$, there exists a sufficiently small disc $\Delta, 0 \in \Delta \subset B$ and a holomorphic map $h: \Delta \longrightarrow D, h(0)=0$, such that $\left(\mathcal{M}_{\Delta}, \Delta, \phi\right)$ is equivalent to $\left(h^{*} \mathcal{N}, \Delta, h^{*} \pi\right)$.

Let $t=\left(t_{1}, \ldots, t_{m}\right)$ where the $t_{i}$ are local complex coordinates at $0 \in B$. Then $\left\langle\frac{\partial}{\partial t_{1}}, \ldots, \frac{\partial}{\partial t_{m}}\right\rangle$ form a basis of $T_{0} B$ and since $\rho_{0}$ is an isomorphism, $\left\langle\left.\frac{\partial M_{t}}{\partial t_{\lambda}}\right|_{t=0} ; 1 \leq \lambda \leq m\right\rangle$ form a basis of $\mathrm{H}^{1}(M, \Theta)$.

Let $h(t)=s=\left(s_{1}, \ldots, s_{\mu}\right)$ where the $s_{j}$ are local complex coordinates at $0 \in D$. Since $(\mathcal{N}, D, \pi)$ is effective implies that it is effective at $0 \in D$, $\left\langle\left.\frac{\partial N_{s}}{\partial s_{\nu}}\right|_{s=0} ; 1 \leq \nu \leq \mu\right\rangle$ are linearly independent in $\mathrm{H}^{1}(M, \Theta)$ where $N_{s}:=$ $\pi^{-1}(s)$. Thus $\mu \leq m$.

On the other hand, by the chain rule (3.3.1.1), the elements $\left\langle\left.\frac{\partial M_{t}}{\partial t_{\lambda}}\right|_{t=0} ; 1 \leq\right.$ $\lambda \leq m\rangle$ (which form a basis of $\mathrm{H}^{1}(M, \Theta)$ ) can be written in terms of the elements $\left\langle\left.\frac{\partial N_{s}}{\partial s_{\nu}}\right|_{s=0} ; 1 \leq \nu \leq \mu\right\rangle$ that are linearly independent in $\mathrm{H}^{1}(M, \Theta)$; this implies $\mu=m$. Thus the number of moduli equals $m=\operatorname{dim}\left(\mathrm{H}^{1}(M, \Theta)\right)$.

Now since $(\mathcal{N}, D, \pi)$ is effective, we have for each $s \in D$ that $\operatorname{dim}\left(\mathrm{H}^{1}\left(N_{s}\right.\right.$, $\left.\left.\Theta_{s}\right)\right) \geq \operatorname{dim}\left(T_{s} D\right)=\mu$. But by the Theorem of Upper-semicontinuity (see assertion (1) of (3.2.11.2)), there exists a neighborhood $\{s \in D ;|s|<\epsilon\}$ of $0 \in D$ such that

$$
\begin{aligned}
|s|<\epsilon \Rightarrow \operatorname{dim}\left(\mathrm{H}^{1}\left(N_{s}, \Theta_{s}\right)\right) & \leq \operatorname{dim}\left(\mathrm{H}^{1}\left(N_{0}, \Theta_{0}\right)\right) \\
& =\operatorname{dim}\left(\mathrm{H}^{1}(M, \Theta)\right)=m=\mu .
\end{aligned}
$$

Hence for $|s|<\epsilon$, we have $\operatorname{dim}\left(\mathrm{H}^{1}\left(N_{s}, \Theta_{s}\right)\right)=\mu$. Thus, the restriction of the family $\mathcal{N}$ to this $\epsilon$-neighborhood of 0 in $D$ is regular.

Choose $\Delta$ sufficiently small so that $h(\Delta) \subset\{s \in D ;|s|<\epsilon\}$. Then $\left(\mathcal{M}_{\Delta}, \Delta, \phi\right)$ is regular because the pullback of a regular family is also regular.

A result depending on the theory of differentiable families of strongly elliptic differential operators asserts that regularity of a family and its effectivity at a point together imply its effectivity in a neighborhood of that point. Hence $\left(\mathcal{M}_{\Delta}, \Delta, \phi\right)$ is effective for $\Delta$ sufficiently small.

Further, for $t \in \Delta$, we have that $\operatorname{dim}\left(\mathrm{H}^{1}\left(M_{t}, \Theta_{t}\right)\right)=\mu=m=\operatorname{dim}\left(T_{t} B\right)$. Thus, for each $t \in \Delta$, we see that $\rho_{t}$ is an isomorphism. Now by the theorem of completeness (3.4.2.3), we see that $\left(\mathcal{M}_{\Delta}, \Delta, \phi\right)$ is an effectively parametrized complete complex analytic family.

This settles the proof of the implication "(a) $\Rightarrow(b)$ " of $(2)$.

The latter part of the above argument settles the converse claim " $(b) \Rightarrow$ (a)" of (2).

Note that all the assertions following (a) and (b) of (2) have also been proved above. 
Proof. (3): We first note that since $\mathrm{H}^{0}(M, \Theta)=\mathrm{H}^{2}(M, \Theta)=0$, by the theorem of upper-semicontinuity we must have $\mathrm{H}^{0}\left(M_{t}, \Theta_{t}\right)=\mathrm{H}^{2}\left(M_{t}, \Theta_{t}\right)=0$ for each $t$ in a sufficiently small neighborhood $\Delta$ of $0 \in B$. It can be shown that if the upper-semicontinuous functions

$$
t \mapsto \operatorname{dim}\left(\mathrm{H}^{i-1}\left(M_{t}, \Theta_{t}\right)\right), \quad t \mapsto \operatorname{dim}\left(\mathrm{H}^{i+1}\left(M_{t}, \Theta_{t}\right)\right)
$$

are independent of $t$ in some neighborhood of a point, then so is the function

$$
t \mapsto \operatorname{dim}\left(\mathrm{H}^{i}\left(M_{t}, \Theta_{t}\right)\right) .
$$

Thus the restricted family $\left(\mathcal{M}_{\Delta}, \Delta, \phi\right)$ is regular QED

3.4.6.2 Remark. In proving the above theorem, Kodaira-Spencer make crucial use of the hypothesis that $\mathrm{H}^{2}(M, \Theta)=0$. The natural question that arises now is whether we can prove a similar theorem for an arbitrary compact complex manifold $M$ i.e., even if $\mathrm{H}^{2}(M, \Theta) \neq 0$. It turns out that there does exist a similar theorem for the general case, but one has to allow the parameter space to be a complex analytic space (see (A.4) and (A.5) of the Appendix) which in general may not be a manifold i.e., it may have singular points. This generalization is due to Kuranishi [14], and we will explain it in (3.6.3). Finally, one can ask more generally whether there is an existence theorem for deformations of the complex analytic space structure on a compact complex analytic space parametrized by another complex analytic space (cf. (3.6.3.1)). It again turns out that there is such a general theorem, proved by Grauert [15], using the Theory of Coherent Analytic Sheaves (the notion of a coherent analytic sheaf is indicated in (A.7.5) of the Appendix).

\subsection{Deformations of Complex Fiber Bundles}

The Theory of Kodaira-Spencer on deformations of compact complex manifolds described so far has been extended by them to the case of deformations of complex fiber bundles. We indicate in this section the definitions relevant to this case and state the main results. For more details, refer to the paper of Kodaira-Spencer [6], chap. III.

\subsubsection{Differentiable and Complex Analytic Families of Complex Fiber Bundles}

3.5.1.1 Definition. Let $(\mathcal{M}, B, \phi)$ be a differentiable family of compact complex manifolds. Let $(\mathbb{B}, \mathcal{M}, \pi)$ be a differentiable fiber bundle over 
$\mathcal{M}$ with structure group a complex Lie group $G$. Let $(\mathbb{P}, \mathcal{M}, \tilde{\pi})$ be the associated principal bundle with both fiber and structure group $G$. Let the complex manifold structure on $\phi^{-1}(t)$ be denoted by $M_{t}$, let $\mathbb{B}_{t}$ denote the restriction of $(\mathbb{B}, \mathcal{M}, \pi)$ to $M_{t}$ and $\mathbb{P}_{t}$ denote the restriction of $(\mathbb{P}, \mathcal{M}, \tilde{\pi})$ to $M_{t}$, for each $t \in B$. We say that $(\mathbb{B}, \mathcal{M}, \pi)$ is a differentiable family of complex fiber bundles over $B$ if $\mathbb{P}_{t}$ is a holomorphic fiber bundle over $M_{t}$ for each $t \in B$.

Notice that, as $t$ varies in $B$, we get various holomorphic fiber bundles $\mathbb{B}_{t}$, but these bundles are defined over different base spaces $M_{t}$. Further, though $(\mathbb{B}, \mathcal{M}, \pi)$ is called a family over $B$, it is actually a bundle over $\mathcal{M}$.

3.5.1.2 Definition. Let $(\mathcal{M}, B, \phi)$ be a complex analytic family of compact complex manifolds. Let $(\mathbb{B}, \mathcal{M}, \pi)$ be a complex analytic fiber bundle over $\mathcal{M}$ i.e., the principal fiber bundle $(\mathbb{P}, \mathcal{M}, \tilde{\pi})$ associated to $(\mathbb{B}, \mathcal{M}, \pi)$ is a holomorphic fiber bundle over $\mathcal{M}$. We call $(\mathbb{B}, \mathcal{M}, \pi)$ a complex analytic family of complex fiber bundles over $B$ if the underlying differentiable fiber bundle of $(\mathbb{B}, \mathcal{M}, \pi)$ is a differentiable family of complex fiber bundles over the underlying differentiable manifold of $B$ in the sense of the previous definition.

3.5.1.3 Definition. Let $(\mathbb{B}, \mathcal{M}, \pi)$ be a differentiable (respectively complex analytic) family of complex fiber bundles over the differentiable (respectively complex analytic) manifold $B$. If the family $\mathcal{M}$ of compact complex manifolds is trivial i.e., it is equivalent to the family $\left(M \times B, B, p_{2}\right)$ where $M$ is a compact complex manifold, then $(\mathbb{B}, \mathcal{M}, \pi)$ is said to be a differentiable (respectively complex analytic) family of complex fiber bundles over $M$ parametrized by $B$; in such a case, if $t^{\prime} \in B$, and if $\mathbb{B}_{t}$ denotes the restriction of the bundle $(\mathbb{B}, \mathcal{M}, \pi)$ to $M_{t}=\pi^{-1}(t) \cong M$, then any bundle $\mathbb{B}_{t}$ considered as a bundle over $M$ is said to be a $C^{\infty}$ (respectively complex analytic) deformation of the bundle $\mathbb{B}_{t^{\prime}}$ over $B$.

3.5.1.4 Example. Let $(\mathcal{M}, B, \phi)$ be a differentiable family of compact complex manifolds and let its fundamental sequence of vector bundles (as in $(3.2 .3))$ be

$$
0 \longrightarrow \mathbb{F} \longrightarrow \mathbb{G} \longrightarrow \mathbb{G} / \mathbb{F} \longrightarrow 0 \text {. }
$$

Recall from (3.2.4) that if $\mathbb{G}_{+}$is the $C^{\infty}$ tangent bundle of $\mathcal{M}$, then $\mathbb{G}$ is a sub-bundle of the complexification $\mathcal{C} \mathbb{G}_{+}$of the first $2 n$ components $(n=$ dimension of any fiber of $\phi$ ) of $\mathbb{G}_{+}$. Further, $\mathbb{F}$ is a direct summand of the complexification $\mathcal{C} \mathbb{F}_{+}$of the sub-bundle $\mathbb{F}_{+}$of $\mathbb{G}_{+}$of tangent vectors along fibers of $\phi$. From the explicit representations of the transition functions that define $\mathbb{F}$ and $\mathbb{G}$ (see $(3.2 .3)$ ), we see that while $\mathbb{F}$ is a differentiable 
family of complex fiber bundles over $(\mathcal{M}, B, \phi), \mathbb{G}$ is not a differentiable family since its structure group is not a complex Lie group.

3.5.1.5 Remark. All the notions defined for families of compact complex manifolds viz. triviality, local triviality, pullback, equivalence, etc., can be analogously defined for families of complex fiber bundles. One can also formulate (cf. (2.3.2.6)) the problems of local moduli and global moduli for deformations of a fixed complex fiber bundle over a compact complex manifold.

\subsubsection{Fundamental Sequences and Diagrams for Families of Complex Fiber Bundles}

Let $(\mathcal{M}, B, \phi)$ be a differentiable family of compact complex manifolds, $(\mathbb{B}, \mathcal{M}, \pi)$ a differentiable family of complex fiber bundles over $B$ and $(\mathbb{P}, \mathcal{M}, \tilde{\pi})$ be the associated differentiable family of complex principal bundles.

The total space $\mathbb{P}$ of the family $(\mathbb{P}, \mathcal{M}, \tilde{\pi})$ can be regarded as a differentiable family of complex manifolds over $B$ in an obvious manner viz. as the family $(\mathbb{P}, B, \phi \circ \tilde{\pi})$ whose fibers are complex analytic principal bundles. Let the fundamental sequence of vector bundles (see (3.2.3)) for this family be given by

$$
0 \longrightarrow \mathbb{F}_{P} \longrightarrow \mathbb{G}_{P} \longrightarrow \mathbb{G}_{P} / \mathbb{F}_{P} \longrightarrow 0 \text {. }
$$

Let $\mathcal{F}_{P}$ be the sub-bundle of $\mathbb{F}_{P}$ composed of those vectors which are tangent to the fibers of $\tilde{\pi}$. Then we also have the following short exact sequence of vector bundles

$$
0 \longrightarrow \mathcal{F}_{P} \longrightarrow \mathbb{G}_{P} \longrightarrow \mathbb{G}_{P} / \mathcal{F}_{P} \longrightarrow 0 \text {. }
$$

Let $G$ be the fiber (and structure group) of $\mathbb{P}$. The group $G$ operates by right translation on $\mathbb{P}$ (where the transition functions operate as usual on the left) and hence it operates on the above short exact sequences. Now let us set

$$
\mathbb{L}=\mathcal{F}_{P} / G, \mathbb{M}=\mathbb{F}_{P} / G \text { and } \mathbb{R}=\mathbb{G}_{P} / G .
$$

Then $\mathbb{L}$ is the bundle over $\mathcal{M}$, with fiber the Lie algebra of $G$ and associated to the principal bundle $(\mathbb{P}, \mathcal{M}, \tilde{\pi})$ obtained by the adjoint action of $G$ on its Lie algebra (which is the tangent space to $G$ at the point representing the identity element of the group operation in $G$ ). Then we obtain the following exact commutative diagram called the fundamental bundle diagram for the family $(\mathbb{P}, \mathcal{M}, \tilde{\pi})$ over $B$ of complex principal bundles: 


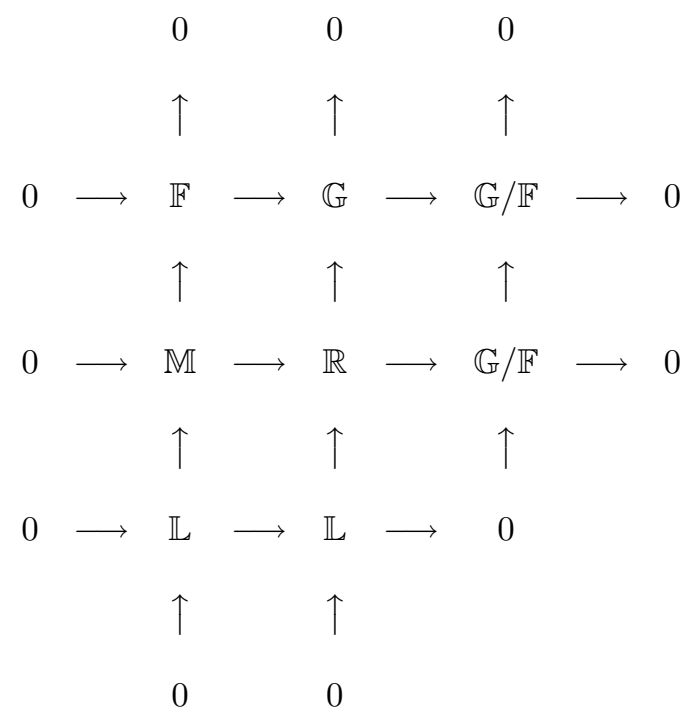

where the short exact sequence

$$
0 \longrightarrow \mathbb{F} \longrightarrow \mathbb{G} \longrightarrow \mathbb{G} / \mathbb{F} \longrightarrow 0
$$

is as usual the fundamental sequence of vector bundles for the family $(\mathcal{M}, B, \phi)$. Next let $\Xi, \Sigma$ and $\Phi$ respectively be sheaves of germs of $C^{\infty}$ sections of $\mathbb{L}, \mathbb{M}$ and $\mathbb{R}$ whose restrictions to each fiber of $\phi$ are holomorphic. Then we get the short exact sequence of sheaves

$$
0 \longrightarrow \Xi \longrightarrow \Phi \stackrel{k}{\longrightarrow} \Psi \longrightarrow 0
$$

where $\Psi$ is the sheaf of germs of $C^{\infty}$ sections of $\mathbb{G}$ whose restrictions to each fiber of $\phi$ are holomorphic. Further let

$$
0 \longrightarrow \Theta \longrightarrow \Pi \stackrel{j}{\longrightarrow} \Upsilon \longrightarrow 0
$$

be the fundamental sequence of sheaves for the family $(\mathcal{M}, B, \phi)$ as defined in (3.2.5). If $\Upsilon_{B}$ is the sheaf associated to the tangent bundle of $B$, then its inverse image sheaf naturally is a subsheaf of the quotient sheaf $\Psi / \Theta$ denoted by $\Upsilon$. Then $\Pi$ is the inverse image of $\Upsilon$ in $\Psi$ under the canonical map $\Psi \longrightarrow \Psi / \Theta$. Let $\Gamma$ be the inverse image sheaf of $\Pi$ under the map $k$ above. Then we get the following short exact sequence of sheaves

$$
0 \longrightarrow \Xi \longrightarrow \Gamma \stackrel{\alpha}{\longrightarrow} \Pi \longrightarrow 0
$$


and we get the following exact commutative diagram of sheaves called the fundamental sheaf diagram for the family $(\mathbb{P}, \mathcal{M}, \tilde{\pi})$ over $B$ of complex principal bundles:

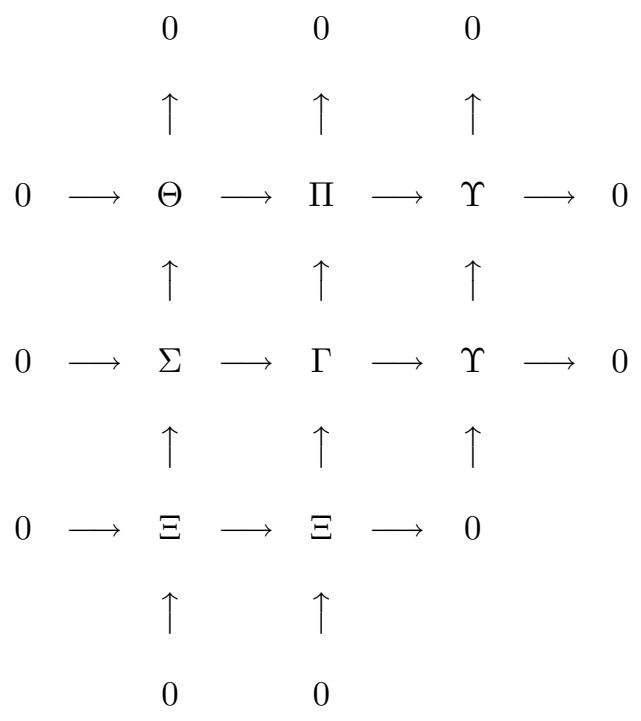

3.5.2.1 Remark. By thinking of any fixed fiber $M_{t}=\phi^{-1}(t)$ as a family over a base space consisting of a single point, we can similarly get fundamental diagrams of vector bundles and sheaves for the fiber over $t \in B$.

\subsubsection{The Global and Infinitesimal Kodaira-Spencer Maps for a Family of Complex Fiber Bundles}

Let $(\mathbb{B}, \mathcal{M}, \pi)$ be a differentiable family of complex fiber bundles over the differentiable family $(\mathcal{M}, B, \phi)$ of compact complex manifolds and $(\mathbb{P}, \mathcal{M}, \tilde{\pi})$ be its associated principal bundle. We continue using the notations of (3.5.2) above. Since sheaf cohomology is a delta functor (see (A.8)), the fundamental sheaf diagram for the associated family $(\mathbb{P}, \mathcal{M}, \tilde{\pi})$ gives rise to an exact commutative cohomology diagram called the deformation diagram for the family of bundles $(\mathbb{B}, \mathcal{M}, \pi)$; this diagram is inset on page 120 . In writing this deformation diagram, we have used the fact that $\mathcal{H}^{0}(\Upsilon) \cong \Upsilon_{B}$. The homomorphism

$$
\eta: \Upsilon_{B} \longrightarrow \mathcal{H}^{1}(\Sigma)
$$

is called the global Kodaira-Spencer map for the family $(\mathbb{B}, \mathcal{M}, \pi)$ on $B$. Of course, $\rho$ is the global Kodaira-Spencer map for the family $(\mathcal{M}, B, \phi)$ as described in (3.2.6). 


\subsubsection{Remarks.}

1. Note that the map $\eta$ is simply the global Kodaira-Spencer map for the differentiable family $(\mathbb{P}, B, \phi \circ \tilde{\pi})$ with the additional restriction that the structure of complex fiber bundle be preserved under deformation.

It is because of this fact that one may expect the results obtained for the global Kodaira-Spencer map for a family of compact complex manifolds, to also hold for the global Kodaira-Spencer map for a family of complex fiber bundles.

2. If $(\mathcal{M}, B, \phi)$ is the trivial family $\left(M \times B, B, p_{2}\right)$ (where $M$ is a compact complex manifold), then the map $\eta$ can be lifted uniquely to a map $\tau: \Upsilon_{B} \longrightarrow \mathcal{H}^{1}(\Xi)$ because of the canonical splitting $\Pi \cong \Theta \oplus \Upsilon$ (cf. $(3.5 .1 .3))$.

3. We could have considered complex analytic families instead of differentiable families above and then we would have arrived at the definition of the global Kodaira-Spencer map for a complex analytic family of complex fiber bundles over a complex analytic family of compact complex manifolds.

4. If we work with a fixed fiber $M_{t}=\phi^{-1}(t), t \in B$, then since we have a corresponding sheaf diagram at $t \in B$, we can similarly obtain the corresponding infinitesimal deformation diagram at $t \in B$ and thus get the following infinitesimal Kodaira-Spencer map for the family of complex fiber bundles in consideration at $t \in B$ (see (3.2.3.1), (3.2.5.1) and (3.2.10.2)):

$$
\eta_{t}: T_{t} B \longrightarrow \mathrm{H}^{1}\left(M_{t}, \Sigma_{t}\right)
$$

Further, when $(\mathcal{M}, B, \phi)$ is isomorphic to the trivial family $\left(M \times B, B, p_{2}\right)$, i.e., when we consider a family of complex fiber bundles over the fixed compact complex manifold $M$ parametrized by $B$, then we get a unique lifting of $\eta_{t}$ to the map

$$
\tau_{t}: T_{t} B \longrightarrow \mathrm{H}^{1}\left(M, \Xi_{t}\right)=\mathrm{H}^{1}\left(M, \mathcal{E} \mathcal{N} \mathcal{D}\left(\mathbb{B}_{t}\right)\right)
$$

where each $M_{t}$ is identified with $M$ and $\mathcal{E N D}$ denotes the sheaf of endomorphisms of the corresponding vector bundle (which is the same thing as the sheaf associated to the endomorphism bundle of the corresponding bundle) (cf. description of $\mathbb{L}$ in (3.5.2)). This discussion applies to both differentiable and complex analytic families of complex fiber bundles.

The following results show that the maps $\eta, \eta_{t}$ are indeed the analogues of the maps $\rho, \rho_{t}$ for the case of families of complex fiber bundles. The results hold irrespective of whether the family of complex fiber bundles in consideration is differentiable or complex analytic. 
Deformation Diagram for $(\mathbb{B}, \mathcal{M}, \pi)$

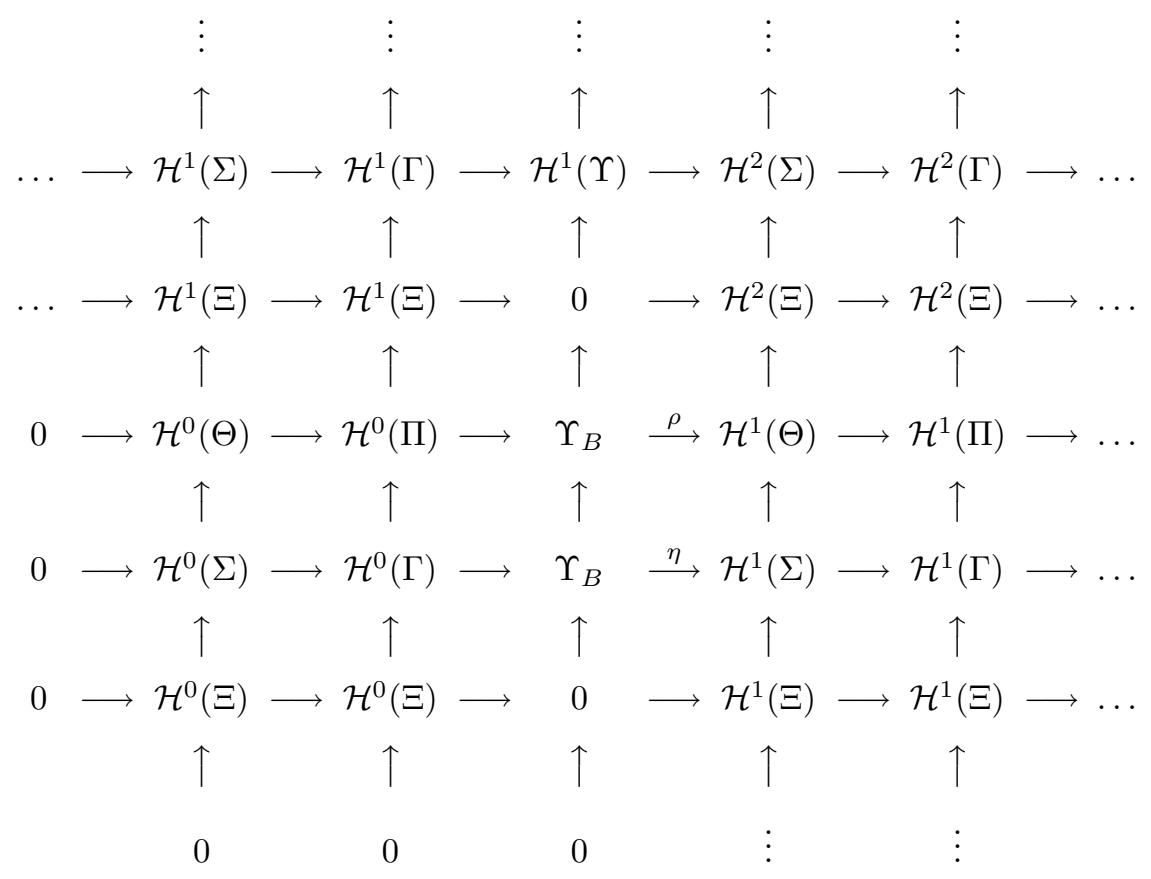

3.5.3.2 Theorem. With the above notations we have the following:

1. The family $(\mathbb{B}, \mathcal{M}, \pi)$ is locally trivial over $B$ if and only if $\eta$ is the zero homomorphism.

2. The family $(\mathbb{B}, \mathcal{M}, \pi)$ is locally trivial over $B$ if it is regular (i.e., the dimension of $H^{1}\left(M_{t}, \Xi_{t}\right)$ is independent of $t \in B$ ) and $\eta_{t}$ vanishes identically for each $t \in B$.

3. If $\eta$ is the zero homomorphism, then $\eta_{t}$ is the trivial linear map for each $t \in B$.

4. (Rigidity Principle) Let $t^{\prime} \in B$ and let $H^{1}\left(M_{t^{\prime}}, \Xi_{t^{\prime}}\right)=0$. Then there exists a neighborhood $U$ of $t^{\prime}$ in $B$ restricted to which $(\mathbb{B}, \mathcal{M}, \pi)$ is trivial.

5. If $(\mathbb{B}, \mathcal{M}, \pi)$ is a family of complex fiber bundles over a fixed compact complex manifold $M$ (i.e., $(\mathcal{M}, B, \phi) \cong\left(M \times B, B, p_{2}\right)$ where $M=$ $\phi^{-1}\left(t^{\prime}\right)=M_{t^{\prime}}$ for some $\left.t^{\prime} \in B\right)$ and if $H^{1}\left(M, \Xi_{t^{\prime \prime}}\right)=0$ for a fixed $t^{\prime \prime} \in B$, then there exists a neighborhood $U$ to $t^{\prime \prime}$ in $B$ restricted to which $(\mathbb{B}, \mathcal{M}, \pi)$ is trivial. 
6. For each $t \in B, \oplus_{q \geq 0} H^{q}\left(M_{t}, \Sigma_{t}\right)$ and $\oplus_{q \geq 0} H^{q}\left(M_{t}, \Xi_{t}\right)$ are graded Lie algebras. Let $[\cdot, \cdot]$ denote the restriction of the Lie algebra multiplication to the first graded pieces. Further let us assume that $(\mathbb{B}, \mathcal{M}, \pi)$ is a family of complex fiber bundles over a fixed compact complex manifold $M$. Then for each point $t \in B$, any element $\zeta \in H^{1}\left(M, \Xi_{t}\right)$ necessarily satisfies $[\zeta, \zeta]=0$ if it corresponds to an infinitesimal deformation of $\mathbb{B}_{t}$.

7. Let $M^{\prime}$ be a compact complex manifold and $\mathbb{B}^{\prime}$ a holomorphic vector bundle such that $H^{2}\left(M^{\prime}, \mathcal{E N} \mathcal{D}\left(\mathbb{B}^{\prime}\right)\right)=0$. Then every element $\zeta \in$ $H^{1}\left(M^{\prime}, \mathcal{E} \mathcal{N} \mathcal{D}\left(\mathbb{B}^{\prime}\right)\right)$ corresponds to an infinitesimal deformation of $\mathbb{B}^{\prime}$. There exists a family of complex vector bundles $(\mathbb{B}, \mathcal{M}, \pi)$ over the trivial family $\left(M^{\prime} \times \Delta, \Delta, p_{2}\right)$ of compact complex manifolds where $\Delta \subset \mathbb{C}^{m}$ is a sufficiently small polydisc with $0 \in \Delta$ such that $\mathbb{B}_{0}=\mathbb{B}^{\prime}$ and further the infinitesimal Kodaira-Spencer map

$$
\tau_{0}: T_{0} \Delta \longrightarrow H^{1}\left(M^{\prime}, \Xi_{0}\right)=H^{1}\left(M^{\prime}, \mathcal{E} \mathcal{N} \mathcal{D}\left(\mathbb{B}^{\prime}\right)\right)
$$

is an isomorphism.

The assertions of the above theorem should be compared with (2) of (3.2.7.1), (2) of (3.2.10.1), (3.2.7.3), (2) of (3.2.8.3), (3) of (3.2.7.1) combined with results of (3.2.9), (3.2.7.5), (3) of (3.2.8.3), (3.4.1.1) and (3.4.6.1).

We describe, in the following sections, the generalisations of the notions of complex analytic family of compact complex manifolds and complex analytic family of complex fiber bundles to the category of complex analytic spaces and also analogues of these notions in the category of schemes.

\subsection{Kuranishi's Theorem and Local Moduli Spaces}

\subsubsection{Deformations of Complex Analytic Spaces}

3.6.1.1 Definition of Analytic Family. Let $B$ be a connected complex analytic space (A.5.4) and let $M_{t}$ be a given complex analytic space for each $t \in B$. We say that $\left\{M_{t} ; t \in B\right\}$ is an analytic family of complex analytic spaces parametrized by $B$ if there exists a morphism $\phi: \mathcal{M} \longrightarrow B$ of complex analytic spaces (A.5.5) such that the following hold:

1. $\phi$ is flat (A.2);

2. $\phi^{-1}(t)=M_{t}$ for each $t \in B$ and 
3. if each $M_{t}$ is a compact complex analytic space, then $\phi$ is proper (A.7.2).

For any fixed complex analytic space $M_{t_{0}}, t_{0} \in B$, any other fiber of $\phi$ viz. $M_{t}$ is called a deformation of $M_{t_{0}}$. B is called the parameter space or the base space and $\mathcal{M}$ is called the total space or deformation space associated to this family. We denote the complex analytic family by $(\mathcal{M}, B, \phi)$.

\subsubsection{Comparison with the Definition of Kodaira-Spencer}

Suppose $(\mathcal{M}, B, \phi)=\left\{M_{t} ; t \in B\right\}$ is an analytic family of compact complex analytic spaces. Suppose in addition that $B, M_{t}(t \in B)$ are all smooth complex analytic spaces i.e., they are all complex manifolds, then it can be shown that $\mathcal{M}$ is itself smooth and that the Jacobian of $\phi$ is of maximal rank at each point of $\mathcal{M}$. Since a smooth complex analytic space is the same as a complex manifold, $(\mathcal{M}, B, \phi)$ becomes a complex analytic family of compact complex manifolds. Conversely, assume that $\psi: \mathcal{N} \longrightarrow T$ is a morphism such that its Jacobian is of maximal rank, $T$ is smooth, the fibers of $\psi$ are smooth and $\psi$ is proper; then it can be shown that $\psi$ is flat, so that $(\mathcal{N}, T, \psi)$ is indeed a complex analytic family of compact complex manifolds. Hence if we want to allow only smooth deformations of a compact complex manifold parametrized by a smooth base space, then the definition of an analytic family $(\mathcal{M}, B, \phi)$ is reduced to requiring that $\phi$ is a proper morphism whose Jacobian is of maximal rank everywhere; in such a case the analytic family becomes a complex analytic family of compact complex manifolds in the sense of Kodaira-Spencer which we stated in (2.4.1.1).

\subsubsection{Analytic Families and Deformation of Complex Analytic Space Structure}

Let $(\mathcal{M}, B, \phi)=\left\{M_{t} ; t \in B\right\}$ be an analytic family of compact complex analytic spaces such that the fiber over each nonsingular point is also nonsingular. Recall that by definition, $B$ is connected. It can then be shown that all fibers are actually topologically isomorphic (more generally, any proper smooth morphism of complex analytic spaces has the property that all the fibers are topologically isomorphic). Of course, if the parameter space is itself nonsingular, then we have seen in this case that all the fibers are actually even diffeomorphic (see (2.3.1.1)).

Thus the notion of an analytic family makes precise the idea of a family of varying complex analytic space structures on a fixed topological space. 


\subsubsection{The Infinitesimal Kodaira-Spencer Maps for an Analytic Family of Complex Analytic Spaces}

In the following discussion we indicate the definition of the infinitesimal Kodaira-Spencer map at a point of the parametrizing complex analytic space only for the case of an analytic family of complex manifolds. For the most general discussion, see [11] and [15].

Let $(\mathcal{M}, B, \phi)$ be an analytic family of compact complex manifolds. We denote the fiber of $\phi$ over $t \in B$ as $M_{t}$. Fix a point $t \in B$. Let the analytic tangent space to $B$ at $t$ be denoted by $T_{t} B$. Define $\mathbb{C}[\epsilon]:=\mathbb{C}[x] /\left(x^{2}\right)$. Any tangent vector $\partial$ (an element of $T_{t} B$ ) corresponds uniquely to a morphism $f=f(\partial)$ of complex analytic spaces, from the first order infinitesimal neighborhood $(\{t\}, \mathbb{C}[\epsilon])$ at $t \in B$, to $B$. Hence, given $\partial$, we get an induced family over $(\{t\}, \mathbb{C}[\epsilon])$ which we denote by $\left(f^{*} \mathcal{M},(\{t\}, \mathbb{C}[\epsilon]), f^{*} \phi\right)$.

Next, using Cech cohomology (A.9), it can be shown that the elements of $\check{\mathrm{H}}^{1}\left(M_{t}, \Theta_{t}\right) \cong \mathrm{H}^{1}\left(M_{t}, \Theta_{t}\right)$ are in bijective correspondence with equivalence classes of families parametrized by $(\{t\}, \mathbb{C}[\epsilon])$ such that the fiber over the closed point of the parameter space is isomorphic to $M_{t}$.

Thus, given an element $\partial$ of $T_{t} B$, we get the morphism $f$ as above and $f$ gives rise to the induced family $\left(f^{*} \mathcal{M},(\{t\}, \mathbb{C}[\epsilon]), f^{*} \phi\right)$ which corresponds to an element $\theta=\theta(f)$ of $\mathrm{H}^{1}\left(M_{t}, \Theta_{t}\right)$ by what we have said in the previous paragraph. Hence we obtain a map

$$
\rho_{t}: T_{t} B \longrightarrow \mathrm{H}^{1}\left(M_{t}, \Theta_{t}\right), \partial \mapsto \theta .
$$

Since $(\{t\}, \mathbb{C}[\epsilon])$ is the first order infinitesimal neighborhood of $t \in B$, the induced family $\left(f^{*} \mathcal{M},(\{t\}, \mathbb{C}[\epsilon]), f^{*} \phi\right)$ can be called an infinitesimal deformation of $M_{t}$ and the above map, which turns out to be a map of $\mathbb{C}$ vector spaces, is called the infinitesimal Kodaira-Spencer map for $(\mathcal{M}, B, \phi)$ at $t \in B$. It can be shown that if $B$ is nonsingular i.e., $B$ is a manifold, then this map coincides with the infinitesimal deformation map for the complex analytic family $(\mathcal{M}, B, \phi)$ as defined earlier in (3.2.8.2).

\subsubsection{Kuranishi's Theorem and Local Moduli Spaces}

The following theorem of Kuranishi [14] is a generalization of the Theorem of Existence (3.4.6.1). Note that we do not assume that $\mathrm{H}^{2}(M, \Theta)=0$. For a complete generalization, see Grauert [15].

3.6.3.1 Theorem of Existence. Let $M$ be a compact complex manifold. Then there exists an analytic family $(\mathcal{M}, B, \phi)=\left\{M_{t}:=\phi^{-1}(t) ; t \in\right.$ $B\}$ of compact complex analytic spaces and a point $t^{\prime} \in B$ with $M_{t^{\prime}}=M$ such that the following hold. 
1. $(\mathcal{M}, B, \phi)$ is complete at $t^{\prime}$ i.e., given any analytic family $(\mathcal{N}, D, \pi)$ with $s^{\prime} \in D$ such that $\pi^{-1}\left(s^{\prime}\right)=M$, there exists a morphism (not necessarily unique) of complex analytic spaces $h: \Delta \longrightarrow B$, where $\Delta$ is a neighborhood of $s^{\prime}$ in $D$ and $h\left(s^{\prime}\right)=t^{\prime}$, such that the family induced by $h$ from $(\mathcal{M}, B, \phi)$ and parametrized by $\Delta$ is equivalent to the restriction of $(\mathcal{N}, D, \pi)$ to $\Delta$.

2. The infinitesimal Kodaira-Spencer map

$$
\rho_{t^{\prime}}: T_{t^{\prime}} B \longrightarrow H^{1}(M, \Theta)
$$

is an isomorphism and the germ of $B$ at $t^{\prime}$ is determined up to a non-canonical isomorphism. $B$ can be chosen to be a complex analytic subset consisting of the common zeros of $l$ holomorphic functions on a polydisc in $\mathbb{C}^{m}$ where $m=\operatorname{dim}_{\mathbb{C}}\left(H^{1}(M, \Theta)\right)$ and $l=$ $\operatorname{dim}_{\mathbb{C}}\left(H^{2}(M, \Theta)\right)$.

3. If $M$ has no "infinitesimal automorphisms", i.e., $H^{0}(M, \Theta)=0$, then the morphism $h$ of (1) above is uniquely determined and the germ of $B$ at $t^{\prime}$ is also uniquely determined up to a canonical isomorphism.

4. If $H^{2}(M, \Theta)=0$, then $t^{\prime}$ is a nonsingular point of $B$ i.e., the complex analytic space structure of $B$ restricted to some neighborhood of $t^{\prime} \in B$ is a manifold. Since $t^{\prime}$ is a smooth point, the dimension of $B$ at $t^{\prime}$ (also equal to the dimension of the germ of $B$ at $t^{\prime}$ ) equals the dimension of $H^{1}(M, \Theta)$ because of (2) above.

5. If $H^{1}(M, \Theta)=0$, then for any analytic family $(\mathcal{N}, D, \pi)$ as in (1) above, there exists a neighborhood of $s^{\prime}$ restricted to which $(\mathcal{N}, D, \pi)$ is a trivial family.

6. If there exist obstructed elements $\theta \in H^{1}(M, \Theta)$ i.e., such that $[\theta, \theta] \in$ $H^{2}(M, \Theta)$ is nonzero, then $B$ has a singularity at $t^{\prime}$.

7. There exists a neighborhood $U$ of $t^{\prime}$ such that $(\mathcal{M}, B, \phi)$ restricted to $U$ is complete (i.e., complete at each point of $U$ ).

The assertions of the above theorem should be compared with results obtained earlier for the smooth category, i.e., with (3) of (3.2.8.3), (3.4.1.1), (3.4.3.2) and (3.4.6.1).

Motivated by the above theorem, we state the following.

3.6.3.2 Definition. With the notations of the above theorem, the germ of $B$ at $t^{\prime}$ is called the local moduli space of $M$. The above theorem says that if $M$ has no infinitesimal automorphisms, then its local moduli space is uniquely determined up to a unique isomorphism; further, if $\mathrm{H}^{2}(M, \Theta)=0$, then this local moduli space is smooth. 


\subsection{Examples of Local Moduli Spaces}

\subsubsection{Riemann's Formula for Moduli: Local Moduli for Curves}

Riemann's formula [16] states that the number of effective independent complex parameters, on which the deformation of complex structure of a compact Riemann surface $M$ of fixed genus $g$ depends, is given by the dimension of the complex Lie group of holomorphic automorphisms of the compact Riemann surface plus the number $(3 g-3)$. Since any compact Riemann surface of genus $g \geq 2$ admits no continuous group of holomorphic automorphisms, we see that the formula for the case $g \geq 2$ is $(3 g-3)$. Of course, when $g=0, M$ has to be biholomorphic to the Riemann sphere $P_{\mathbb{C}}^{1}$. For an elementary proof of this, see [17], chap.V, theorem 8. So the number of moduli of $M$ is zero (notice that the number of moduli of $P_{\mathbb{C}}^{n}$ is also zero for any positive integer $n$ - see (3) of (3.2.8.3)). Next when $g=1, M$ is an elliptic curve and the number of moduli is 1 as shown in sec. 1.5 of chap. 1 or in (3.4.4.1). We assume that $g \geq 2$ in the following discussion.

By Kuranishi's theorem (3.6.3.1), a local moduli space for $M$ exists. Since the (complex) dimension of $M$ is 1 , we have that $\mathrm{H}^{2}(M, \mathcal{F})=0$ for any sheaf $\mathcal{F}$ of abelian groups on $M$ (say by a result of Grothendieck). Thus $\mathrm{H}^{2}(M, \Theta)=0$, which implies that the local moduli space of $M$ is smooth and therefore has dimension equal to

$$
\operatorname{dim}\left(\mathrm{H}^{1}(M, \Theta)\right)=\operatorname{dim}\left(\mathrm{H}^{0}(M, \mathcal{K} \otimes \mathcal{K})\right)
$$

because of Serre Duality where $\mathcal{K}$ is the canonical sheaf of $M$ i.e., the sheaf associated to the holomorphic cotangent bundle $K$ (which is the dual $T^{*}(M)$ of the tangent bundle $T(M)$ ) of $M$ (see (4.4.2.2)). Alternatively, we may also obtain this by directly using the theorem of existence (3.4.6.1) of Kodaira-Spencer without appealing to Kuranishi's theorem.

We will now use the following result: let $L$ be a holomorphic line bundle on a compact Riemann surface $X$ of genus $g \geq 2$, and let the degree of the line bundle $L$ (cf. (4.6.3.1)) be greater than $(2 g-1)$; we then necessarily have $\mathrm{H}^{1}(X, \mathcal{L})=0$, where $\mathcal{L}$ is the sheaf of germs of holomorphic sections of the line bundle $L$ (for a proof of this result, see [18], (2.7)). Now using the Riemann-Roch Theorem (see (4.6.3.5)) we have that

$$
\begin{aligned}
\operatorname{degree}(K) & =\operatorname{dim}\left(\mathrm{H}^{0}(M, \mathcal{K})\right)-\operatorname{dim}\left(\mathrm{H}^{1}(M, \mathcal{K})\right)+(\operatorname{rank}(K)) \cdot(g-1) \\
& =g-\operatorname{dim}\left(\mathrm{H}^{0}\left(M, \mathcal{O}_{M}\right)\right)+(g-1)=2 g-2,
\end{aligned}
$$

where we have used the definition of genus and Serre Duality (see (4.4.2.4)). 
Since we have (see (4.6.3.4))

$$
\operatorname{degree}(K \otimes K)=2 \cdot \operatorname{degree}(K)=2(2 g-2) \geq(2 g-1),
$$

we get that $\operatorname{dim}\left(\mathrm{H}^{1}(M, \mathcal{K} \otimes \mathcal{K})\right)=0$. Again using the Riemann-Roch theorem we have that the number of moduli of $M$ is given by

$$
\begin{aligned}
\operatorname{dim}\left(\mathrm{H}^{0}(M, \mathcal{K} \otimes \mathcal{K})\right) & =\operatorname{dim}\left(\mathrm{H}^{1}(M, \mathcal{K} \otimes \mathcal{K})\right)+\operatorname{degree}(K \otimes K)-g+1 \\
& =0+(4 g-4)-g+1=3 g-3
\end{aligned}
$$

Hence the formula of Riemann coincides with our calculations. Note also that, according to Teichmüller Theory, the number of moduli of $M$ is given by $\operatorname{dim}\left(\mathrm{H}^{0}(M, \mathcal{K} \otimes \mathcal{K})\right)$ (see [19]). Teichmüller's theory relates to the study of the elements of $\mathrm{H}^{0}(M, \mathcal{K} \otimes \mathcal{K})$. These are locally defined by expressions of the form

$$
\psi_{j}=\psi_{j}\left(z_{j}\right) \cdot d z_{j} \otimes d z_{j}
$$

where $\left(U_{j}, z_{j}\right)$ is a local complex coordinate chart on $M$ and $\psi_{j}\left(z_{j}\right)$ is a holomorphic function on $U_{j}$. Such elements are called holomorphic quadratic differentials.

\subsubsection{An Example where the Local Moduli Space has Singularities}

Let $T^{n}$ be an $n$-dimensional complex torus $(n \geq 2)$. Consider the product compact complex manifold $M:=T^{n} \times P_{\mathbb{C}}^{1}$. Then Kodaira-Spencer show in [6], chap.VI, sec.16, that there exists an element $\theta \in \mathrm{H}^{1}(M, \Theta)$ such that the element $[\theta, \theta] \in \mathrm{H}^{2}(M, \Theta)$ is nonzero. Hence the corresponding family $(\mathcal{M}, B, \phi)$ of Kuranishi's theorem (3.6.3.1) with $\phi^{-1}\left(t^{\prime}\right)=M$ has a singularity at $t^{\prime} \in B$ (see assertion (6) of (3.6.3.1)). Thus the germ of $B$ at $t^{\prime}$ cannot be smooth.

This example shows us why we need to allow possibly non-smooth complex-analytic spaces as parameter spaces even if our primary aim was to study only smooth deformations of a smooth complex manifold.

\subsubsection{Local Moduli Spaces for Complex Tori}

Let $M$ be a complex $n$-dimensional torus. We have seen in (3.4.4.1) that there exists a complete effectively parameterized complex analytic family $(\mathcal{B}, S, \phi)$ of deformations of $M$. We have also seen in (3.4.4) that since the infinitesimal Kodaira-Spencer map is an isomorphism at each point of $S$ for this family (that is how we showed that it is effectively parametrized and complete), we have $\operatorname{dim}\left(\mathrm{H}^{1}(M, \Theta)\right)=n^{2}=\operatorname{dim}(S)$. Hence the germ 
of $S$ at each point $s \in S$ is the local moduli space of the corresponding torus $M_{s}:=\phi^{-1}(s)$. Since $S$ is smooth, the dimension of each of these local moduli spaces is $n^{2}$. Of course, if $n \geq 2$ we have seen in (2.6.2) that we do not get an injective parametrization.

\subsection{Deformations and Local Moduli for Vector Bundles over a Compact Riemann Surface}

\subsubsection{Families of Vector Bundles and the Infinitesimal Deformation Maps}

\subsubsection{Family of Vector Bundles}

Let $X$ be a compact Riemann surface and $V=(V, X, \pi)$ a holomorphic vector bundle on $X$ of rank $n$ and degree $d$ (see (4.6.3.3)). By an analytic family of vector bundles of rank $n$ and degree $d$ over $X$ parametrized by a complex analytic space $B$ is meant a complex analytic vector bundle $\mathbb{V}$ on the product complex analytic space $X \times B$ such that for each $t \in B$, the pullback $\mathbb{V}_{t}$ of $\mathbb{V}$ to $X$ under the canonical inclusion

$$
X \cong X \times\{t\} \hookrightarrow X \times B
$$

is a holomorphic vector bundle of rank $n$ and degree $d$ over $X$. If there exists a point $t^{\prime} \in B$ such that $\mathbb{V}_{t^{\prime}}$ is isomorphic to $V$, then we call $\mathbb{V}_{t}$ a deformation of $V$ over $B$ for each $t \in B$. When $B$ is smooth, this definition coincides with the one given in (3.5.1.3).

\subsubsection{Infinitesimal Deformation Maps for a Family}

We continue with the notations of (3.8.1.1) above. Let $(\mathbb{V}, X \times B, \phi)$ be an analytic family of holomorphic vector bundles over the compact Riemann surface $X$ parametrized by the complex analytic space $B$. This is by definition a vector bundle on $X \times B$ and we shall simply denote it by $\mathbb{V}$. We denote the restriction of $\mathbb{V}$ to the fiber $X \cong X \times\{t\}$ by $\mathbb{V}_{t}$ which is a holomorphic vector bundle over $X$ and call it the fiber of $\mathbb{V}$ over $t \in B$.

Fix a point $t \in B$. Let the analytic tangent space to $B$ at $t$ be denoted by $T_{t} B$. Any tangent vector $\partial$ (an element of $T_{t} B$ ) corresponds uniquely to a morphism $f=f(\partial)$ of complex analytic spaces, from the first order infinitesimal neighborhood $(\{t\}, \mathbb{C}[\epsilon])$ at $t \in B$, to $B$. We thus get an induced analytic family of holomorphic vector bundles over $X$ parametrized by $(\{t\}, \mathbb{C}[\epsilon])$ which we denote by $f^{*} \mathbb{V}$. 
Next, using Čech cohomology (A.9), it can be shown that the elements of

$$
\check{\mathrm{H}}^{1}\left(X, \mathcal{E} \mathcal{N} \mathcal{D}\left(\mathbb{V}_{t}\right)\right) \cong \mathrm{H}^{1}\left(X, \mathcal{E} \mathcal{N} \mathcal{D}\left(\mathbb{V}_{t}\right)\right)
$$

are in bijective correspondence with equivalence classes of families parametrized by $(\{t\}, \mathbb{C}[\epsilon])$ for each of which the corresponding fiber over the closed point of this parameter space is isomorphic to $\mathbb{V}_{t}$. Here $\mathcal{E N D}\left(\mathbb{V}_{t}\right)$ denotes the (locally free) sheaf over $X$ of germs of endomorphisms of the bundle $\mathbb{V}_{t}$.

Thus given an element $\partial$ of $T_{t} B$, we have the morphism $f$ as above, and this gives rise to the induced family $f^{*} \mathbb{V}$ which corresponds to an element $\theta=\theta(f)$ of $\mathrm{H}^{1}\left(X, \mathcal{E} \mathcal{N} \mathcal{D}\left(\mathbb{V}_{t}\right)\right)$ by what we have said in the previous paragraph. Hence we obtain a map

$$
\tau_{t}: T_{t} B \longrightarrow \mathrm{H}^{1}\left(X, \mathcal{E} \mathcal{N} \mathcal{D}\left(\mathbb{V}_{t}\right)\right), \partial \mapsto \theta .
$$

Since $(\{t\}, \mathbb{C}[\epsilon])$ is the first order infinitesimal neighborhood of $t \in B$, the induced family $f^{*} \mathbb{V}$ can be called an infinitesimal deformation of $\mathbb{V}_{t}$ and the above map, which turns out to be a map of $\mathbb{C}$-vector spaces, is called the infinitesimal Kodaira-Spencer map for the family $\mathbb{V}$ at $t \in B$. It can be shown that if $B$ is nonsingular i.e., $B$ is a manifold, so that this family becomes a complex analytic family of holomorphic vector bundles, then the infinitesimal deformation map for this complex analytic family as defined earlier in (3.5.3) coincides with the above map.

\subsubsection{Local Moduli for Simple Vector Bundles over a Compact Riemann Surface}

Let $X$ be a compact Riemann surface and let $V$ be a holomorphic vector bundle over $X$ such that it has no nontrivial endomorphisms i.e., any morphism (of holomorphic vector bundles) of $V$ into itself is just given by "multiplication by a complex number"; this is the same as requiring that $H^{0}(X, \mathcal{E} \mathcal{N} \mathcal{D}(V)) \cong \mathbb{C}$. Such bundles $V$ are said to be simple. So let $V$ be a simple bundle of rank $n$ over $X$. Line bundles are always simple and simple bundles of arbitrary rank exist if the genus of the compact Riemann surface $X$ is greater than 1 .

The analogue of Kuranishi's theorem (3.6.3.1) is also true for families of holomorphic vector bundles over any fixed compact complex manifold and parametrized by complex analytic spaces. Thus there exists an analytic family of holomorphic vector bundles $(\mathbb{V}, X \times B, \phi)$ parametrized by a complex analytic space $B$ with $t^{\prime} \in B$ such that the fiber of $\mathbb{V}$ over $t^{\prime}$, which is a holomorphic vector bundle over $X$, is isomorphic (as holomorphic vector bundle) to $V$ and further such that the infinitesimal deformation map

$$
\tau_{t^{\prime}}: T_{t^{\prime}} B \longrightarrow \mathrm{H}^{1}(X, \mathcal{E} \mathcal{N} \mathcal{D}(V))
$$


is an isomorphism. For the existence of such a family for the case $n=1$ of line bundles, see (4.6.6). Since $X$ is of (complex) dimension 1 , we have that $\mathrm{H}^{2}(X, \mathcal{E} \mathcal{N} \mathcal{D}(V))=0$. Thus we see that the point $t^{\prime} \in B$ must be a nonsingular point of $B$ so that we actually get (locally at $t^{\prime}$ ) a complex analytic family.

Therefore also we see that the dimension of the local moduli space (which by definition is the germ of $B$ ) at $t^{\prime} \in B$ must be equal to the dimension of the cohomology group $\mathrm{H}^{1}(X, \mathcal{E N D}(V))$. We will compute this dimension below.

To begin with, we note that the degree of the holomorphic vector bundle $\operatorname{END}(V)$ (whose associated sheaf of germs of holomorphic sections over $X$ is precisely the sheaf $\mathcal{E N D}(V))$ is zero as the following calculation shows (see (4.6.3.4)).

$$
\begin{aligned}
\operatorname{deg}(\operatorname{END}(V)) & =\operatorname{deg}\left(V \otimes V^{*}\right)=(\operatorname{rank}(V)) \cdot\left(\operatorname{deg}(V)+\operatorname{deg}\left(V^{*}\right)\right) \\
& =(\operatorname{rank}(V)) \cdot(\operatorname{deg}(V)-\operatorname{deg}(V))=0 .
\end{aligned}
$$

We have used above the fact that just as in the case of vector spaces, we have an isomorphism of $\operatorname{END}(V)$ with $V \otimes V^{*}$. Using the Riemann-Roch theorem for vector bundles (see (4.6.3.5)) we have the required dimension of the local moduli space for $V$ as

$$
\begin{aligned}
& \operatorname{dim}\left(\mathrm{H}^{1}(X, \mathcal{E N} \mathcal{D}(V))\right) \\
& \quad=\operatorname{dim}\left(\mathrm{H}^{0}(X, \mathcal{E N} \mathcal{D}(V))\right)-\operatorname{deg}(\mathcal{E N} \mathcal{D}(V))+(\operatorname{rank}(\operatorname{END}(V))) \cdot(g-1) \\
& \quad=1-0+n^{2}(g-1)=n^{2}(g-1)+1
\end{aligned}
$$

where $g$ is the genus of $X$. Thus the total number of independent effective complex parameters, on which the structure of a simple holomorphic vector bundle imposed on the fixed underlying topological vector bundle of $V$ depends, is $n^{2}(g-1)+1$.

It can also be shown that in this moduli problem, one obtains an injective parametrization unlike the case of tori (2.6.2).

\subsection{Deformation of Schemes and Geometric Vector Bundles over Schemes}

In this section, we will freely use the terminology explained in the Appendix. We begin with the definition of algebraic deformation of a nonsingular scheme of finite type over $\mathbb{C}$ and also of an algebraic vector bundle (a coherent locally free sheaf of fixed rank (see (4.6.1))) over such a scheme parametrized by a scheme over $\mathbb{C}$. Of course, by a scheme over $\mathbb{C}$, we mean a scheme 
together with a morphism from it into the one-point-scheme Spec $(\mathbb{C})$. Further, we always work only with closed points.

\subsubsection{Algebraic Families of Schemes and Vector Bundles}

3.9.1.1 Definition. An (algebraic) family of schemes of finite type over $\mathbb{C}$ parametrized by a (connected) scheme $B$ over $\mathbb{C}$ is a morphism $\phi: \mathcal{M} \longrightarrow B$ of schemes over $\mathbb{C}$ such that:

1. $\phi$ is flat;

2. $\phi$ is of finite type i.e., if $U=\operatorname{Spec}(R)$ is any affine open subscheme of $B$, then $\phi^{-1}(U)$ is a union of finitely many affine open subschemes $V_{i}=\operatorname{Spec}\left(S_{i}\right)$ of $\mathcal{M}$ such that the natural homomorphisms of rings $R \longrightarrow S_{i}$ (which actually define $\phi$ locally) make each $S_{i}$ into a finitely generated $R$-algebra;

3. if $t \in B$ is a closed point such that the scheme theoretic fiber $M_{t}:=$ $\mathcal{M} \times{ }_{B} \operatorname{Spec}(k(t))$-where $k(t)$ is the residue field at $t$, i.e., the quotient of the local ring at $t$ by the unique maximal ideal of that ringwith underlying topological space $\phi^{-1}(t)$ is proper over $\mathbf{S p e c}(\mathbb{C})$, then $\phi$ is required to be a proper morphism.

The family is denoted by $(\mathcal{M}, B, \phi)=\left\{M_{t} ; t \in B\right\}$.

For any scheme $M$ and a family $(\mathcal{M}, B, \phi)=\left\{M_{t} ; t \in B\right\}$ as above with a point $t^{\prime} \in B$ such that $M_{t^{\prime}} \cong M$, we call any other scheme theoretic fiber $M_{t}$ as a (global algebraic) deformation of $M$ parametrized by $B$. The scheme $\mathcal{M}$ is called the deformation space or the total space and $B$ the parameter space or base space for this family of deformations.

\subsubsection{Remarks.}

1. Let $(\mathcal{M}, B, \phi)$ be a family of schemes of finite type over $\mathbb{C}$ and let $B$ also be a scheme of finite type over $\mathbb{C}$. Then by (A.10) we get an associated analytic family of complex analytic spaces parametrized by a complex analytic space in the sense of definition (3.6.1.1).

2. Alternative Formulation Involving Jacobians. Suppose $(\mathcal{M}, B, \phi)=\left\{M_{t} ; t \in B\right\}$ is an algebraic family of complete smooth separated integral schemes of finite type over $\mathbb{C}$ with the parameter space $B$ also smooth over $\mathbb{C}$. Here by a complete scheme over $\mathbb{C}$ we mean a scheme over $\mathbb{C}$ whose morphism into $\operatorname{Spec}(\mathbb{C})$ (which makes this scheme a scheme over $\mathbb{C}$ ) is a proper morphism. Of course, a scheme of finite type over $\mathbb{C}$ is complete if and only if the associated complex analytic space is compact (see (A.10.4.1)). Under these 
additional hypotheses, it can be shown that $\mathcal{M}$ is itself smooth and that the Jacobian of $\phi$ is of maximal rank at each point of $\mathcal{M}$ (i.e., the differential of the map $\phi$ is surjective at each point of $\mathcal{M})$. Conversely, if we assume that $\psi: \mathcal{N} \longrightarrow T$ is a morphism of schemes over $\mathbb{C}$ such that its Jacobian is of maximal rank, $T$ is smooth, the fibers of $\psi$ are smooth and $\psi$ is proper then it can be shown that $\psi$ is flat, so that $(\mathcal{N}, T, \psi)$ is indeed an algebraic family of smooth schemes over $\mathbb{C}$. Hence if we want to allow only smooth deformations parametrized by a smooth base space, then the definition of a family is simply a proper morphism whose Jacobian is of maximal rank everywhere. In this respect also compare with (3.6.1.2).

3.9.1.3 Definition. Let $X$ be a scheme of finite type over $\mathbb{C}$. By an algebraic vector bundle over $X$ of rank $n$, we mean a coherent locally free sheaf of $\mathcal{O}_{X}$-modules of rank $n$ over $X$ (see the beginning paragraph of (4.6.1)). Of course, by the GAGA principle (A.10.5.1), if $X_{H O L}$ is a compact Riemann surface, then the sheaf associated to any holomorphic vector bundle of rank $n$ over $X_{H O L}$ corresponds to a unique algebraic vector bundle of rank $n$ over $X$ and conversely.

3.9.1.4 Remarks. Just as in the case of holomorphic vector bundles over a complex manifold, we may define the following notions for the category of schemes of finite type over $\mathbb{C}$ :

1. a family of algebraic vector bundles over a smooth scheme parametrized by a scheme;

2. a global algebraic deformation of an algebraic vector bundle over a smooth scheme parametrized by a scheme;

3. equivalence of algebraic families of vector bundles over a fixed smooth scheme and parametrized by a fixed scheme;

4. triviality and local triviality of an algebraic family of vector bundles, and

5. pullback of algebraic families under morphisms of schemes.

Of course, due to definition (3.9.1.3), all the above definitions would involve only coherent locally free sheaves and familiar operations on such sheaves. For example, the pullback of an algebraic vector bundle (or even of a family of algebraic vector bundles) corresponds to the inverse image of the corresponding coherent locally free sheaf as defined in (A.3) which turns out again to be coherent and locally free. 


\subsubsection{Infinitesimal Deformation Maps for Algebraic Families of Schemes}

Let $X$ be a nonsingular irreducible separated scheme of finite type over $\mathbb{C}$ of dimension $n$. Let $\Omega_{X / \mathbb{C}}$ be its sheaf of Kähler differentials as defined in (4.4.2.2). Let us define the tangent sheaf by

$$
\Theta=\mathcal{H} \mathcal{O} \mathcal{M}_{\mathcal{O}_{X}}\left(\Omega_{X / \mathbb{C}}, \mathcal{O}_{X}\right),
$$

where $\mathcal{H} \mathcal{O} \mathcal{M}_{\mathcal{O}_{X}}(\mathcal{F}, \mathcal{G})$ denotes the sheaf of $\mathcal{O}_{X}$-module homomorphisms of $\mathcal{F}$ into $\mathcal{G}$ i.e., the sheaf associated to the presheaf on $X$ given by $U \mapsto$ $\operatorname{HOM}_{\mathcal{O}_{X}(U)}(\mathcal{F}(U), \mathcal{G}(U))$ where $U$ is an open subset of $X$. We also define the canonical sheaf of $X$ by

$$
\omega_{X}=\Lambda^{n}\left(\Omega_{X / \mathbb{C}}\right),
$$

where $\Lambda^{n}\left(\Omega_{X / \mathbb{C}}\right)$ denotes the $n$-th exterior power of the sheaf $\Omega_{X / \mathbb{C}}$. Then $\Omega_{X / \mathbb{C}}, \Theta$ and $\omega_{X}$ are coherent locally free sheaves of ranks $n, n$ and 1 respectively.

Using Cech cohomology, we can show that the elements of the cohomology group

$$
\check{\mathrm{H}}^{1}(X, \Theta) \cong \mathrm{H}^{1}(X, \Theta)
$$

are in a natural bijective correspondence with equivalence classes of triples $(\mathcal{M}, \operatorname{Spec}(\mathbb{C}[\epsilon]), \phi)$ of algebraic families over $\operatorname{Spec}(\mathbb{C}[\epsilon])$ such that the fiber product scheme $\mathcal{M} \times_{\operatorname{Spec}(\mathbb{C}[\epsilon])} \operatorname{Spec}(\mathbb{C})$ is isomorphic to $X(\mathbb{C}[\epsilon]$ was defined in $(3.6 .2))$.

Now we come to the definition of the algebraic analogue of the infinitesimal Kodaira-Spencer map. Let $(\mathcal{M}, B, \phi)$ be an algebraic family of nonsingular irreducible schemes of finite type over $\mathbb{C}$ and let $t^{\prime} \in B$ be a closed point such that the scheme theoretic fiber of $\phi$ over this point is isomorphic to $X$. Hence $(\mathcal{M}, B, \phi)$ is an algebraic family of global deformations of $X$ with base point $t^{\prime} \in B$.

Given any element $\partial$ of the Zariski tangent space $T_{t^{\prime}} B$, we get a unique morphism $f: \operatorname{Spec}(\mathbb{C}[\epsilon]) \longrightarrow B$ mapping onto $t^{\prime} \in B$. Then we have the induced family $f^{*} \mathcal{M}$ over $\operatorname{Spec}(\mathbb{C}[\epsilon])$ which gives an element $\theta=\theta(f)$ of $\mathrm{H}^{1}(X, \Theta)$ as indicated earlier. Define the map

$$
\rho_{t^{\prime}}: T_{t^{\prime}} B \longrightarrow \mathrm{H}^{1}(X, \Theta) \text { by } \partial \mapsto \theta .
$$

Since $\mathbf{S p e c}(\mathbb{C}[\epsilon])$ is the first order infinitesimal neighborhood of $t^{\prime} \in B$, the above family corresponding to $\partial$ on $\operatorname{Spec}(\mathbb{C}[\epsilon])$ is called as the infinitesimal deformation of $X$ along the tangent direction at $t^{\prime} \in B$ prescribed by $\partial$.

The map $\rho_{t^{\prime}}$ is called the infinitesimal deformation map at $t^{\prime} \in B$ for the algebraic family under consideration and is the analogue of the KodairaSpencer map (3.6.2) for a family of complex manifolds. 
Of course, when $X$ is projective over $\mathbb{C}$, by the GAGA principle (A.10.5.1), the underlying complex analytic space of $X$ viz. $X_{H O L}$ (A.10.1) is a compact complex manifold, the sheaf associated to $\Theta$ of $\mathcal{O}_{X_{H O L}}$ modules viz. $\Theta_{H O L}$ (A.10.2) is precisely the sheaf of germs of holomorphic vector fields on $X_{H O L}$ and the Zariski tangent space $T_{t^{\prime}} B$ gets canonically identified with the complex analytic tangent space of $B_{H O L}$ at $t^{\prime}$. Then by (4) of (A.10.5.1), the infinitesimal Kodaira-Spencer map for the associated complex analytic family at $t^{\prime} \in B_{H O L}$ is the same as $\rho_{t^{\prime}}$ above.

\subsubsection{Infinitesimal Deformation Maps for Algebraic Families of Algebraic Vector Bundles}

Let $X$ be a smooth scheme of finite type over $\mathbb{C}$. Let $\mathcal{V}$ be an algebraic vector bundle of rank $n$ on $X$ i.e., $\mathcal{V}$ is a coherent locally free sheaf of $\mathcal{O}_{X}$-modules of rank $n$ (3.9.1.3). of $\mathcal{V}$

Let $\mathcal{E} \mathcal{N} \mathcal{D}(\mathcal{V})$ denote the sheaf of germs of $\mathcal{O}_{X}$-module endomorphisms

Using Cech cohomology, we can show that the elements of the cohomology group

$$
\check{\mathrm{H}}^{1}(X, \mathcal{E} \mathcal{N} \mathcal{D}(\mathcal{V})) \cong \mathrm{H}^{1}(X, \mathcal{E N} \mathcal{D}(\mathcal{V}))
$$

are in bijective correspondence with isomorphism classes of coherent locally free sheaves $\mathcal{W}$ on the product scheme $X \times \mathbf{S p e c}(\mathbb{C}[\epsilon])$ such that the inverse image $\mathcal{O}_{X}$-module of $\mathcal{W}$ under the canonical morphism

$$
X \cong X \times \operatorname{Spec}(\mathbb{C}) \stackrel{1_{X} \times \phi}{\longrightarrow} X \times \operatorname{Spec}(\mathbb{C}[\epsilon])
$$

(where $\phi$ is the closed immersion of the closed point of Spec $(\mathbb{C})$ into Spec $(\mathbb{C}[\epsilon]))$ is isomorphic to $\mathcal{V}$. Such $\mathcal{W}$ are algebraic families of algebraic deformations of $\mathcal{V}$ parametrized by $\operatorname{Spec}(\mathbb{C}[\epsilon])$ and hence are called infinitesimal deformations of $\mathcal{V}$.

Next, let $\mathcal{U}$ be an algebraic family of algebraic (rank $n$ ) vector bundles over $X$ parametrized by a scheme $B$ of finite type over $\mathbb{C}$. Fix a closed point $t \in B$. Let $\mathcal{U}_{t}$ denote the algebraic vector bundle of rank $n$ on $X$ got by pulling back $\mathcal{U}$ under the canonical morphism

$$
X \cong X \times\{t\} \stackrel{1_{X} \times \phi_{t}}{\longrightarrow} X \times B
$$

where $\phi_{t}:\{t\} \hookrightarrow B$ is the closed immersion of the closed point $t$ into $B$.

Given any element $\partial$ of the Zariski tangent space $T_{t} B$, we get a unique associated morphism $f: \operatorname{Spec}(\mathbb{C}[\epsilon]) \longrightarrow B$ mapping onto $t \in B$. Then we have the induced family $f^{*} \mathcal{U}$ over $\operatorname{Spec}(\mathbb{C}[\epsilon])$ which gives an element $\theta=\theta(f)$ of $\mathrm{H}^{1}\left(X, \mathcal{E} \mathcal{N} \mathcal{D}\left(\mathcal{U}_{t}\right)\right)$ as indicated earlier. Define the map

$$
\tau_{t}: T_{t} B \longrightarrow \mathrm{H}^{1}\left(X, \mathcal{E} \mathcal{N} \mathcal{D}\left(\mathcal{U}_{t}\right)\right) \text { by } \partial \mapsto \theta .
$$


The map $\tau_{t}$ is called the infinitesimal deformation map at $t \in B$ for the algebraic family under consideration and is the analogue of the Kodaira-Spencer map for a family of holomorphic vector bundles over a fixed complex manifold.

Of course, when $X$ is projective over $\mathbb{C}$, by the GAGA principle, the infinitesimal Kodaira-Spencer map for the associated complex analytic family at $t \in B_{H O L}$ is the same as $\tau_{t}$ above.

Deformation Theory in the context of schemes is explained in detail in the book of Sernesi [38] and of Hartshorne [41].

We will not go into the technical definition of an "algebraic local moduli space". For details, see Schlessinger's paper [20]. However, see (3) of (4.6.7.3) and (4.7.3.6).

In the next chapter, we study the notion of a "global moduli space". 


\section{Theory of Global Moduli: Fine Moduli Spaces and Coarse Moduli Spaces}

\subsection{Overview of this Chapter}

The notion of a family is fundamental to the formulation of every moduli problem. We have already illustrated the notion of a family of varying complex structures in chapter 2, and we have studied the local properties of such families in the previous chapter. The general definition of a family, which can be used to formulate a general moduli problem, is explained in section 4.2.

Problems of moduli of complex structures are of the following two major types: the problem of the variation of the complex manifold structure on the underlying topological space of a compact complex manifold, and the problem of the variation of the holomorphic vector bundle structure on the underlying topological vector bundle of a holomorphic vector bundle over a compact complex manifold.

The problem of moduli of a compact connected complex manifold is explained in subsection 4.3.1. The simplest case of this problem is that corresponding to a compact connected complex manifold of dimension 1, which is a compact Riemann surface. Among the compact Riemann surfaces, the more simpler ones are the tori, which correspond to compact Riemann surfaces of genus 1. Thus, the problem of moduli of elliptic curves is the simplest example of a problem of variation of complex manifold structures, and this example is explained in subsection 4.3.3. Though this moduli problem is easily formulated, its solution involves nontrivial theory, as shown by sections 4.7 and 4.8 . 
Subsection 4.3.2 explains the formulation of the problem of moduli of a holomorphic vector bundle on a compact Riemann surface.

The definition of a fine moduli space given in subsection 4.5.3 involves the notion of a representable functor. Hence, the notion of a representable functor is introduced earlier in subsection 4.4.1, and is illustrated with examples in subsection 4.4.2. These naturally occurring examples serve the following additional purpose: they are used to define the canonical sheaf and the tangent sheaf to a nonsingular curve. These objects, alongwith the Serre Duality Theorem and the Riemann-Roch Theorem, were already used in computations in subsections 3.7.1 and 3.8.2 of the previous chapter.

The general notion of a family, defined in section 4.2 , is used to define a general moduli problem in functorial terms in subsection 4.5.1.

The best possible solution to a moduli problem, called a fine moduli space, is defined in subsection 4.5.3. The abstract nature of this functorial definition is clarified and made more transparent by the notion of a universal family. The exact relationship between a fine moduli space and a universal family is explained in 4.5.3.3.

A coarse moduli space is a less better solution to a moduli problem than a fine moduli space, but in many moduli problems this is the best we are able to get. The notion of a coarse moduli space is defined in subsection 4.5.4. The functoriality of the definition involved is convenient only to state the definition and to see immediately that every fine moduli space is also a coarse moduli space. However, this definition is not practically useful and hence an alternative definition is indicated. This version is easier to apply, and in fact, is used to obtain the coarse moduli space for elliptic curves in section 4.7 .

There are necessary and sufficient conditions that can ensure that a coarse moduli space is actually a fine moduli space. These conditions are explained in 4.5 .5 .

The method of passing from the local moduli spaces to a global moduli space is illustrated in the constructions of sections 4.6 and 4.7.

Given a compact Riemann surface of genus $g$, the abelian group of isomorphism classes of line bundles of degree zero, with the tensor product as the group operation, can be canonically given the structure of a complex $g$-dimensional torus. This torus is called the Jacobian of the given compact Riemann surface and it turns out to be algebraizable. The aim of section 4.6 is to construct the Jacobian and show that it is a fine moduli space for the problem of moduli of degree zero holomorphic line bundles on the given compact Riemann surface. The associated universal family is called the Poincaré family, which is a complete effectively and injectively parametrized family. 
The rank and the degree of a holomorphic vector bundle on a compact Riemann surface are integer invariants of the underlying topological bundle. Hence these are fixed for the problem of the variation of the holomorphic structure of the bundle.

The definition of degree of a vector bundle involves the notion of the Chern class of a line bundle. In order to define Chern classes, one has to first identify isomorphism classes of line bundles with the elements of the first cohomology group of the compact Riemann surface with values in the sheaf of units associated to the sheaf of rings given by its structure sheaf. Hence, the Picard group of a ringed space is defined in subsection 4.6.1, following which, in subsection 4.6.2, Chern classes are defined.

The notion of the degree of a holomorphic vector bundle and its properties are explained in subsection 4.6.3. The famous Riemann-Roch Theorem is also stated there.

The Jacobian is constructed in subsection 4.6.5, the properties of the Poincaré family are discussed next in 4.6.6 and finally in 4.6.7, it is established that the Jacobian is a universal classifying space.

The problem of moduli of elliptic curves admits a coarse moduli space for its solution, but does not possess a fine moduli space. That the space of complex numbers is a coarse moduli space for this problem, is the content of section 4.7 .

The elliptic modular function, defined on the upper-half complex plane and associated to the natural family of elliptic curves parametrized by this upper-half plane, can be generalized to any arbitrary family of elliptic curves. This generalization is the crucial step in solving this moduli problem, and is dealt with in subsection 4.7.2. The coarse moduli space for elliptic curves is obtained, using this generalization, in 4.7.3.

What are the reasons behind the non-existence of a fine moduli space for elliptic curves? Answers to this question are given in section 4.8.

Subsection 4.8.1 explains how there exist local obstructions to the existence of tautological families at those points of the parameter space which correspond to elliptic curves admitting more automorphisms than a general elliptic curve.

There exist families of elliptic curves, which have isomorphic fibers, but yet are not globally trivial. The existence of such families implies the impossibility of obtaining a fine moduli space and hence such families are called global obstructions. The construction of such a family is sketched in subsection 4.8.2. 


\subsection{The General Definition of a Family}

\subsubsection{Classification Problems and Families}

Suppose we are given a set $S$ of objects of a fixed type in Geometry and an equivalence relation $\sim_{S}$ on $S$. Then we may form the set $S / \sim_{S}$ of equivalence classes. The set-theoretic classification problem is to find some discrete invariants of objects of $S$ such as maps $f: S / \sim_{S} \longrightarrow \mathbb{Z}$ which partition $S / \sim_{S}$.

A structural classification problem attempts to put some "mathematical structure" on $S / \sim_{S}$. A structural classification problem can be said to be a "well-formulated" classification problem if the structure on $S / \sim_{S}$ relates satisfactorily and in a natural way to that on the elements of $S$.

Given a "mathematical structure" which we want to put on $S / \sim_{S}$, we look at the category $\mathcal{C}$ whose objects are sets alongwith the given "mathematical structure" and whose morphisms are maps of underlying sets that "preserve" (or suitably relate to) the chosen mathematical structure. Our aim primarily is to see if the elements of the underlying set $|M|$ of a suitable object $M$ of $\mathcal{C}$ are in a natural bijective correspondence with the elements of the set $S / \sim_{S}$, so that we may "transport the structure" on $|M|$ to $S / \sim_{S}$.

Next, we require this structure to be naturally related to the structures on elements of $S$. This requirement may be made more precise by the notion of a "family of elements of $S$ parametrized by an object of $\mathcal{C}$ " which we define below. For a "well-formulated" classification problem, we want the structure on $S / \sim_{S}$ to reflect properties of "families of objects of $S$ parametrized by objects of $\mathcal{C}$ ". Before making the definition of a family, we will assume that the category $\mathcal{C}$ satisfies certain conditions which we state below.

\subsubsection{Conditions on the Parameter Category $\mathcal{C}$}

The category $\mathcal{C}$ will be assumed to have the following properties:

1. The objects of $\mathcal{C}$ are sets with some additional mathematical structure and the morphisms in $\mathcal{C}$ are maps of the underlying sets relating suitably to the mathematical structure. Further, the underlying map of a composition (in a given order) of a given finite set of morphisms in $\mathcal{C}$ is the composition of the underlying maps corresponding to the given morphisms in the same order. The collection of morphisms from any object to any object in $\mathcal{C}$ is always a set.

2 . Let $\mathcal{S}$ denote the category whose objects are sets and whose morphisms are set-theoretic maps. Define the covariant functor $|\cdot|$ : $\mathcal{C} \longrightarrow \mathcal{S}$ by sending an object $M$ of $\mathcal{C}$ to its underlying set $|M|$, and 
by sending a morphism $f: M \longrightarrow N$ in $\mathcal{C}$ to the underlying mapping of sets $|f|:|M| \longrightarrow|N|$. Then for each pair of objects $N, M \in \mathcal{C}$, the mapping of sets induced by the above functor

$$
\operatorname{Hom}_{\mathcal{C}}(M, N) \longrightarrow \operatorname{Hom}_{\mathcal{S}}(|M|,|N|)
$$

must be injective (here $\operatorname{Hom}_{\mathcal{A}}(M, N)$ denotes the collection of morphisms from an object $M$ to another object $N$ in a given category $\mathcal{A}$ ). Another way of saying this is that a morphism in $\mathcal{C}$ is uniquely determined if the corresponding map of the underlying sets is uniquely determined.

3. There exists a base point object denoted by $P \in \mathcal{C}$ such that its underlying set $|P|$ consists of a single point and there exists a canonical identification of the set $\operatorname{Hom}_{\mathcal{C}}(P, M)$ with $|M|$ for each $M \in \mathcal{C}$ in the sense that if $f: M \longrightarrow N$ is a morphism in $\mathcal{C}$, then the underlying map of sets $|f|:|M| \longrightarrow|N|$ is just given by the natural map

$$
\operatorname{Hom}_{\mathcal{C}}(P, M) \longrightarrow \operatorname{Hom}_{\mathcal{C}}(P, N)
$$

defined by $\psi \rightarrow f \circ \psi$. We are now ready to make the following definition.

4.2.3 Definition. We continue with the same notations as above i.e., let $S$ be a set of objects in Geometry and $\sim_{S}$ be an equivalence relation on this set $S$. Let $\mathcal{C}$ be a category satisfying (4.2.2). The classification problem is to put on the set $S / \sim_{S}$ the structure of an object of $\mathcal{C}$ in a natural manner. A functor of families of objects of $S$ is a contravariant functor $\mathcal{F} \mathcal{A M}: \mathcal{C} \longrightarrow \mathcal{S}$ which satisfies the following conditions:

1. $\mathcal{F} \mathcal{A M}(P)=S$;

2. for each object $T$ of $\mathcal{C}$, there is given an equivalence relation $\sim_{T}$ on the set $\mathcal{F} \mathcal{A M}(T)$ such that when $T=P, \sim_{T}$ reduces to the already given equivalence relation $\sim_{S}$;

3. for each morphism $\phi: T_{1} \longrightarrow T_{2}$ in $\mathcal{C}$, the corresponding map of sets

$$
\mathcal{F} \mathcal{A M}(\phi): \mathcal{F} \mathcal{A M}\left(T_{2}\right) \longrightarrow \mathcal{F} \mathcal{A M}\left(T_{1}\right)
$$

takes $\sim_{T_{2}}$-equivalent families to $\sim_{T_{1}}$-equivalent families.

\subsubsection{Remarks on the Above Definition}

1. For any object $T \in \mathcal{C}$, the elements of $\mathcal{F} \mathcal{A M}(T)$ are called families of objects of $S$ parametrized by $T$. 
2. Hence (1) of the above definition says that families parametrized by the base point object $P$ are just objects of $S$ themselves. Further, condition (2) of the above definition says that the equivalence relation on families parametrized by $P$ is just the equivalence on $S$ already given.

3. For a morphism $\phi: T_{1} \longrightarrow T_{2}$ in $\mathcal{C}$, the corresponding map of sets

$$
\mathcal{F} \mathcal{A M}(\phi): \mathcal{F} \mathcal{A M}\left(T_{2}\right) \longrightarrow \mathcal{F} \mathcal{A M}\left(T_{1}\right)
$$

is denoted by $\phi^{*}$. For any family $F$ parametrized by $T_{2}$, the family $\phi^{*} F$ is called the pullback to $T_{1}$ of $F$ by $\phi$. It is also called the family on $T_{1}$ induced by $\phi$. The operation $\phi \mapsto \phi^{*}$ is functorial by definition. Further, if $F$ is a family parametrized by $T \in \mathcal{C}$ and if $t$ is a point of the underlying set $|T|$ of $T$, since there is a canonical identification $|T|=\operatorname{Hom}_{\mathcal{C}}(P, T)$ due to which we think of $t$ as a morphism $\phi_{t}: P \longrightarrow T$ in $\mathcal{C}$, then the element of $S$ given by $\phi_{t}^{*}(F)$ is called the fiber of $F$ over $t$ and is often denoted by $F_{t}$.

4. Condition (3) of the above definition may also be stated as follows: the pullback operation on families is compatible with the equivalence relations on the respective families.

\subsubsection{The Functor of Equivalence Classes of Families}

Given a functor $\mathcal{F} \mathcal{A M}$ of families of objects of $S$ as in (4.2.3) above, since we want to classify objects of $S$ up to $\sim_{S}$-equivalence, we can define a contravariant functor called the functor of equivalence classes of families of objects of $S$ as follows. This is the functor $\mathcal{F}: \mathcal{C} \longrightarrow \mathcal{S}$ defined by $\mathcal{F}(T)=$ $\mathcal{F} \mathcal{A M}(T) / \sim_{T}$; in other words, for each object $T \in \mathcal{C}, \mathcal{F}(T)$ is the set of $\sim_{T}$-equivalence classes of families of objects of $S$ parametrized by $T$. Notice that $\mathcal{F}(P)=S / \sim_{S}$.

Next let $\phi: T_{1} \longrightarrow T_{2}$ be a morphism in $\mathcal{C}$ and

$$
\phi^{*}=\mathcal{F} \mathcal{A M}(\phi): \mathcal{F} \mathcal{A M}\left(T_{2}\right) \longrightarrow \mathcal{F} \mathcal{A M}\left(T_{1}\right)
$$

be the corresponding map of sets. Then we define the mapping $\mathcal{F}(\phi)$ : $\mathcal{F}\left(T_{2}\right) \longrightarrow \mathcal{F}\left(T_{1}\right)$ by sending the $\sim_{T_{2}}$-equivalence class, of a family $F$ parametrized by $T_{2}$, to the $\sim_{T_{1}}$-equivalence class, of the family $\phi^{*}(F)$ parametrized by $T_{1}$. This is well-defined because the pullback operation on families is compatible with the equivalence on families.

Thus we get the functor $\mathcal{F}$ of equivalence classes of families of objects of $S$ parametrized by objects of $\mathcal{C}$.

In the next section, we illustrate how functors like $\mathcal{F} \mathcal{A M}$ and $\mathcal{F}$ arise naturally in situations involving classification problems. 


\subsection{Examples of Functors of Families}

\subsubsection{Example: Problem of Moduli of a Compact Complex Manifold}

Let $M$ be a compact connected complex manifold. Let $M_{C^{\infty}}$ denote the underlying differentiable manifold of $M$. We wish to study those structures of complex manifold that can be imposed on $M_{C^{\infty}}$ which also occur as deformations of the complex structure $M$ on $M_{C^{\infty}}$.

Let $S$ denote the set of pairs of the form $\left(M_{C^{\infty}},\left\{\left(U_{j}, z_{j}\right) ; j \in \mathbb{N}\right\}\right)$ where $\left\{\left(U_{j}, z_{j}\right) ; j \in \mathbb{N}\right\}$ is a system of local complex coordinates on $M_{C^{\infty}}$ that endows $M_{C^{\infty}}$ with the structure of a complex manifold which occurs as a deformation of $M$.

We define the equivalence relation $\sim_{S}$ on $S$ by declaring two elements of $S$ to be equivalent if and only if they give rise to biholomorphic complex structures on $M_{C^{\infty}}$.

This discussion applies to both complex analytic families and differentiable families. Therefore the words "family", "deformation", "manifold", "map of manifolds" should be replaced throughout by the words "complex analytic family", "holomorphic deformation", "complex manifold", "holomorphic map" respectively, or throughout by the words "differentiable family", " $C^{\infty}$ deformation", "differentiable manifold", " $C$ map" respectively.

Let $\mathcal{C}$ denote the category whose objects are pairs of the form $\left(B, t_{0}\right)$ where $B$ is a connected manifold and $t_{0}$ a point of $B$ called the base point and whose morphisms are maps of manifolds that preserve base points. Let $\mathcal{S}$ denote the category whose objects are sets and whose morphisms are settheoretic maps. Define the association $\mathcal{F} \mathcal{A M}: \mathcal{C} \longrightarrow \mathcal{S}$ as in (2.5.1). As checked in (2.5.1), it is clear that $\mathcal{F} \mathcal{A M}$ is a functor of families of objects of $S$ parametrized by objects of $\mathcal{C}$ that naturally gives rise to the functor $\mathcal{F}$ of equivalence classes of families.

\subsubsection{Example: Problem of Moduli of Vector Bundles on a Compact Riemann Surface}

Fix a compact Riemann surface $X$. Let $S$ denote the set of isomorphism classes of holomorphic vector bundles on $X$ of fixed rank $n$ and fixed degree $d$ (see (4.6.3.3)). Let $\sim_{S}$ denote "equality in $S$ " so that $S / \sim_{S}=S$.

Suppose we want to bijectively parametrize isomorphism classes of holomorphic vector bundles (in $S$ ) by a Hausdorff reduced complex analytic space (see (A.7.1)). Then we consider the category $\mathcal{C}$ whose objects are Hausdorff reduced complex analytic spaces and whose morphisms are morphisms 
of complex analytic spaces. For the base point object $P \in \mathcal{C}$, take the nonsingular complex analytic space (manifold) consisting of a single point $|P|=\{p\}$. Then $\mathcal{C}$ is a category satisfying (4.2.2).

Define a family of holomorphic vector bundles in $S$ parametrized by $T \in \mathcal{C}$ to be the isomorphism class $[V]$ of a holomorphic vector bundle $V=(V, X \times$ $\left.T, p_{V}\right)$ of rank $n$ on $X \times T$ such that for each point $t=\phi_{t} \in|T| \leftrightarrow \phi_{t} \in$ $\operatorname{Hom}_{\mathcal{C}}(P, T)$ (see (3) of (4.2.2)), the pullback of this vector bundle to $X$ by the following composition

$$
X \cong X \times P \stackrel{1_{X} \times \phi_{t}}{\longleftrightarrow} X \times T
$$

(which is a holomorphic vector bundle on $X$ of rank $n$ denoted by $V_{t}$ ) has degree $d$. Next, for each $T \in \mathcal{C}$, define the equivalence relation $\sim_{T}$ on $\mathcal{F} \mathcal{A M}(T)$ by $\left[V_{1}\right] \sim_{T}\left[V_{2}\right] \Leftrightarrow V_{1}$ is isomorphic to the tensor product $V_{2} \otimes p_{T}^{*}(L)$ where $p_{T}: X \times T \longrightarrow T$ is the canonical projection onto the second factor, $L$ is a holomorphic line bundle on $T$, and $p_{T}^{*}(L)$ is the pullback of $L$ by $p_{T}$ to $X \times T$.

The motivation for the above definition of equivalence $\sim_{T}$ is given by the following non-trivial result.

4.3.2.1 "See-Saw" Theorem. Let $X$ be a connected compact complex manifold (respectively an integral separated scheme of finite type over $\mathbb{C}$ such that the structure morphism $X \longrightarrow \operatorname{Spec}(\mathbb{C})$ is proper $)$. Let $T$ be any reduced complex analytic space (respectively any reduced scheme of finite type over $\mathbb{C}$ ). Let $L$ be a holomorphic line bundle (respectively an algebraic line bundle - a coherent locally free sheaf of rank 1) over the product $X \times T$. Then the subset $T_{0}$ of $T$ defined by

$$
T_{0}=\left\{\text { closed points } t \in T ;\left(1_{X} \times \phi_{t}\right)^{*}(L) \text { is trivial on } X \times P \cong X\right\},
$$

is an analytic subset (respectively a Zariski-closed subset) of $T$. Further if $p_{2}^{0}: X \times T_{0} \longrightarrow T_{0}$ is the canonical projection onto the second factor and $i_{0}: T_{0} \longrightarrow T$ is the canonical inclusion, then we must have the following isomorphism of holomorphic (respectively algebraic) line bundles on $X \times T_{0}$ :

$$
\left(1_{X} \times i_{0}\right)^{*}(L) \cong\left(p_{2}^{0}\right)^{*}(M),
$$

where $M$ is some holomorphic (respectively algebraic) line bundle on $T_{0}$.

For the proof of this theorem in the complex analytic category, see [21] (Appendix A); for a proof in the algebraic category see [9], (chap. II, sec. 5).

We now explain the implication of the above theorem regarding the choice of an equivalence relation on families. 
We consider the simplest case viz. $n=1$ corresponding to the moduli problem of holomorphic line bundles of fixed degree.

For classes $\left[V_{1}\right]$ and $\left[V_{2}\right]$ of holomorphic line bundles in $S$ parametrized by $T \in \mathcal{C}$, since the representatives $V_{1}$ and $V_{2}$ are by definition holomorphic line bundles on $X \times T$, we are naturally tempted to define

$\left[V_{1}\right] \sim_{T}\left[V_{2}\right]$ if and only if $V_{1} \cong V_{2}$ as holomorphic line bundles.

Of course, in such a case, it is clear that for each $t \in|T|$, we will have $\left(V_{1}\right)_{t} \cong\left(V_{2}\right)_{t}$ as holomorphic line bundles on $X$. However, conversely if $W_{1}$ and $W_{2}$ are respectively representatives of two families of holomorphic line bundles $\left[W_{1}\right]$ and $\left[W_{2}\right]$ in $S$ parametrized by $T \in \mathcal{C}$ such that they are fiberwise isomorphic i.e., $\left(W_{1}\right)_{t} \cong\left(W_{2}\right)_{t}$ for each point $t$ of $T$, then due to the above theorem, we get only that

$$
W_{1} \cong W_{2} \otimes p_{T}^{*}(M)
$$

for some holomorphic line bundle $M$ on $T$. Therefore $W_{1}$ and $W_{2}$ need not be isomorphic as holomorphic line bundles on $X \times T$. This shows that our definition of $\sim_{T}$ as isomorphism of representative holomorphic line bundles is more restrictive than our original definition.

Later on, we shall define a "fine moduli space" (in (4.5.3)) which is the best global solution one could hope to get to a moduli problem. In case a fine moduli space exists (this is indeed the case for holomorphic line bundles of degree zero - see (4.6.7.2)), we shall show later (in (4.5.3.3.3)) that the equivalence relation on families is determined by the equivalence relation on $S$. Therefore, with the definition of $\sim_{T}$ as isomorphism of representative holomorphic line bundles, we would be unable to get a fine moduli space.

Therefore, the above discussion justifies our original definition of $\sim_{T}$ namely

$$
\left[W_{1}\right] \sim_{T}\left[W_{2}\right] \Leftrightarrow W_{1} \cong W_{2} \otimes p_{T}^{*}(M)
$$

for some holomorphic line bundle $M$ on $T$.

For a morphism $\phi: T_{1} \longrightarrow T_{2}$ in $\mathcal{C}$, we define the pullback $\phi^{*}[V]$ of an element $[V] \in \mathcal{F} \mathcal{A} \mathcal{M}\left(T_{2}\right)$ as follows: $\phi^{*}[V]:=\left[\left(1_{X} \times \phi\right)^{*}(V)\right] \in \mathcal{F} \mathcal{A} \mathcal{M}\left(T_{1}\right)$. It is then routinely verified that $\mathcal{F} \mathcal{A M}$ is indeed a functor of families of vector bundles is $S$ parametrized by Hausdorff reduced complex analytic spaces and hence gives rise to the functor $\mathcal{F}$ of equivalence classes of families given by $\mathcal{F}(T)=\mathcal{F} \mathcal{A} \mathcal{M}(T) / \sim_{T}$.

4.3.2.2 Remark. In the above discussion, if we replace "holomorphic vector bundle" by "algebraic vector bundle", "complex analytic space" by "reduced separated scheme of finite type over $\mathbb{C}$ ", "morphism of complex 
analytic spaces" by "morphism of schemes over $\mathbb{C}$ " respectively and take $P=\operatorname{Spec}(\mathbb{C}), \mathcal{C}$ to be the category of reduced separated schemes of finite type over $\mathbb{C}$, and work only with closed points (i.e., for $T \in \mathcal{C},|T|$ denotes only the closed points of the underlying topological space of $T$ ), then we get verbatim a functor of families naturally arising in Algebraic Geometry. Note that $X$ is algebraizable and since each holomorphic vector bundle on $X$ corresponds to a locally free sheaf (it corresponds to its sheaf of germs of sections), by the GAGA principle (A.10.5.1), we see that each holomorphic vector bundle on $X$ has a unique structure of algebraic vector bundle over the algebraic scheme structure on $X$.

\subsubsection{Example: Problem of Moduli of Elliptic Curves}

Recall from chapter 1 that a compact Riemann surface of genus 1 is called an elliptic curve and recall from (1.5.2.6.2) that it can be embedded as a closed complex submanifold of 2-dimensional complex projective space $P_{\mathbb{C}}^{2}$. By the GAGA principle (A.10.5.1), (the conformal equivalence class of) an elliptic curve actually corresponds to (the algebraic isomorphism class of) a projective algebraic scheme over $\operatorname{Spec}(\mathbb{C})$ viz. a projective nonsingular irreducible 1-dimensional scheme over $\operatorname{Spec}(\mathbb{C})$, which also we shall refer to as an elliptic curve (over $\operatorname{Spec}(\mathbb{C})$ ).

Let $S$ be the set whose elements are pairs of the form $(E, e)$ where $E$ is an elliptic curve and $e \in E$ is a closed point.

Let $\sim_{S}$ be the equivalence relation on $S$ given by $(E, e) \sim_{S}\left(E^{\prime}, e^{\prime}\right) \Leftrightarrow$ there exists an isomorphism of $E$ onto $E^{\prime}$ carrying $e$ onto $e^{\prime}$. Since each elliptic curve comes with an algebraic group structure, we see that the group of automorphisms of an elliptic curve is transitive, so that $S / \sim_{S}$ is precisely the set of isomorphism classes of elliptic curves.

Suppose we want to bijectively parametrize isomorphism classes of elliptic curves (elements of $S / \sim_{S}$ ) by a Hausdorff reduced complex analytic space (A.7.1). Then we consider the category $\mathcal{C}$ whose objects are Hausdorff reduced complex analytic spaces and whose morphisms are morphisms of complex analytic spaces (A.5.5). For the base point object $P \in \mathcal{C}$, take the nonsingular complex analytic space (manifold) consisting of a single point i.e., $|P|=\{p t\}$. Then $\mathcal{C}$ is a category satisfying (4.2.2).

Following (3.6.1.1), we define a family of elliptic curves parametrized by $T \in \mathcal{C}$ to be an analytic family $(F, T, p)$ together with a section $s$ of $p$, where $F \in \mathcal{C}$, the morphism $p: F \longrightarrow T$ (in $\mathcal{C}$ ) is proper, flat and with each fiber $p^{-1}(t),(t \in T)$ an elliptic curve. We may, of course, equivalently require that $p$ be proper and smooth instead of requiring that $p$ be proper and flat.

When $T$ is nonsingular, i.e., a manifold, then $F$ is also a manifold and the differential of the map $p$ is of maximal rank at each point of $F$ so that 
the above definition reduces to that of Kodaira-Spencer (see (2.4.1.1) and (3.6.1.2)).

We shall denote a family $p: F \longrightarrow T$ alongwith the given section $s$ as above simply by $(F, s)$ and denote (for each $t \in T$ ) its fiber $p^{-1}(t)$ by $\left(F_{t}, s(t)\right) \in S$.

Thus $\mathcal{F} \mathcal{A M}(T)$ is the set of families of elliptic curves parametrized by $T \in \mathcal{C}$ alongwith prescribed sections.

Let the equivalence relation $\sim_{T}$ on $\mathcal{F} \mathcal{A M}(T)$ be given by $(F, s) \sim_{T}$ $\left(F^{\prime}, s^{\prime}\right) \Leftrightarrow F$ and $F^{\prime}$ are equivalent as families over $T$ by an isomorphism of $F$ onto $F^{\prime}$ which when composed with $s$ gives $s^{\prime}$.

Given a family $(F, s) \in \mathcal{F} \mathcal{A M}(T)$ and a morphism $\phi: T^{\prime} \longrightarrow T$ in $\mathcal{C}$, we define the pullback of the family $(F, s)$ by $\phi$, denoted $\phi^{*}(F, s) \in \mathcal{F} \mathcal{A M}\left(T^{\prime}\right)$ to be $\left(T^{\prime} \times{ }_{T} F, \phi^{*} s\right)$ where $\left(T^{\prime} \times{ }_{T} F\right)$ is the fibered product (A.7.3) of $T^{\prime}$ and $F$ over $T$ with respect to the maps $p$ and $\phi$ and where $\phi^{*} s$ is the section of $\phi^{*} p$ induced by $s$.

It is routinely verified that $\mathcal{F} \mathcal{A M}$ is indeed a contravariant functor of families from $\mathcal{C}$ to $\mathcal{S}$, which naturally gives rise to the functor $\mathcal{F}$ of equivalence classes of families from $\mathcal{C}$ to $\mathcal{S}$, defined by $\mathcal{F}(T)=\mathcal{F} \mathcal{A M}(T) / \sim_{T}$.

4.3.3.1 Remark. In the above discussion, if we take for $\mathcal{C}$ the category of separated reduced schemes over $\mathbb{C}, P:=\operatorname{Spec}(\mathbb{C})$, let $|T|$ for $T \in \mathcal{C}$ denote only the closed points of $T$, and consider algebraic families of elliptic curves over $\operatorname{Spec}(\mathbb{C})$ parametrized by objects of $\mathcal{C}$ to be families in the sense of definition (3.9.1.1) alongwith prescribed sections, then we obtain the contravariant functors $\mathcal{F} \mathcal{A M}$ and $\mathcal{F}$ from $\mathcal{C}$ to $\mathcal{S}$ respectively of families and of equivalence classes of families of elliptic curves over $\mathbb{C}$.

We have already indicated in (2.5.2) the notion of a moduli problem in terms of the notion of a functor of families. The best kind of solution that one may expect for a moduli problem is called a fine moduli space. In order to understand clearly what this term means, we need to understand the notion of a representable functor which is what we describe in the next section. Further, for many moduli problems, one does not get a fine moduli space but what one often gets is another type of solution to the moduli problem called a coarse moduli space. In order to motivate this concept, we need Yoneda's Lemma which is purely a category-theoretic result and is also proved in the next section.

\subsection{Representable Functors}

\subsubsection{Yoneda's Lemma}

The notion of a representable functor is inspired by the intuition that the "structure" of an object in a "good" category (e.g., a category satisfying 
(4.2.2)) must be completely prescribed by the knowledge of all ("allowed maps") morphisms into it from every other object of that category. This is in fact true and the result is called Yoneda's lemma which we prove in this section in (4.4.1.4).

The relevance of the notion of a representable functor to classification problems is the following. From a well-formulated classification problem arises a functor $\mathcal{F}$ which when evaluated at a "base-point-space" equals the set of equivalence classes of objects on which we want to discover a "good structure" (cf. (4.3)). Now consider the category $\mathcal{C}$ of objects with the "good structure". Every object in this category gives rise to a representable functor (4.4.1.2). Furthermore, this functor evaluated at a "base-pointspace" gives back the underlying set of the object which it represents (recall (3) of (4.2.2)). Now if the functor arising from the classification problem is isomorphic to such a functor defined by an object of the category, then the structure on the set of equivalence classes of objects that we want to classify (transported from the structure on the object of the category) "behaves well" with respect to $\mathcal{F}$ in the sense that we get a "universal family" - see (4.5.3.3). We begin with the following.

4.4.1.1 Definition. Let $\mathcal{C}$ be a category. Note that if $X \in \mathcal{C}$ is an object of $\mathcal{C}$, then

$$
\operatorname{Hom}_{\mathcal{C}}(-, X): \mathcal{C} \longrightarrow \mathcal{S}
$$

is a contravariant functor, where $\mathcal{S}$ denotes the category whose objects are sets and whose morphisms are set-theoretic mappings, and $\operatorname{Hom}_{\mathcal{C}}(X, Y)$ denotes the collection of morphisms from $X$ to $Y$ in $\mathcal{C}$. We define a contravariant functor $F: \mathcal{C} \longrightarrow \mathcal{S}$ to be representable if there exists a natural equivalence (i.e., isomorphism of functors):

$$
\Phi: F \stackrel{\cong}{\longrightarrow} \operatorname{Hom}_{\mathcal{C}}(-, X)
$$

for some object $X$ of $\mathcal{C}$. We also say that the pair $(X, \Phi)$ represents $F$. That the pair $(X, \Phi)$ is uniquely defined up to a unique isomorphism (if it exists) will be implied by Yoneda's lemma (4.4.1.5).

4.4.1.2 Remark. For any $X \in \mathcal{C}$, the functor $F_{X}:=\operatorname{Hom}_{\mathcal{C}}(-, X)$ is representable by definition. It is represented by the pair $\left(X, 1_{F_{X}}\right)$ where $1_{F_{X}}$ is the identity natural equivalence on $F_{X}$.

4.4.1.3 Definition. Let $\mathcal{C}$ be a category and $X$ be an object of $\mathcal{C}$. Let $\operatorname{FUNCT}\left(\mathcal{C}^{o}, \mathcal{S}\right)$ denote the category whose objects are covariant functors, from $\mathcal{C}^{o}\left(\mathcal{C}^{\circ}\right.$ is just the category $\mathcal{C}$ with arrows reversed) to $\mathcal{S}$, and whose morphisms are natural transformations of functors. This is a full subcategory of the category of (covariant) functors. Define the functor 


$$
h: \mathcal{C} \longrightarrow \operatorname{FUNCT}\left(\mathcal{C}^{o}, \mathcal{S}\right)
$$

by $X \mapsto h_{X}$ where $h_{X}: \mathcal{C}^{o} \longrightarrow \mathcal{S}$ is the covariant functor which is defined by $Y \mapsto h_{X}(Y):=\operatorname{Hom}_{\mathcal{C}}(Y, X) \in \mathcal{S}$.

4.4.1.4 Lemma (Yoneda). Let $\mathcal{C}$ be a category and $X$ an object of $\mathcal{C}$. The we have:

(a) if $F: \mathcal{C} \longrightarrow \mathcal{S}$ is any contravariant functor, then the natural transformations of functors from $\operatorname{Hom}_{\mathcal{C}}(-, X)$ to $F$ are in a natural bijective correspondence with the elements of $F(X)$;

(b) the functor $h$ (defined above) is an equivalence of $\mathcal{C}$ with a full subcategory of the category of functors.

Proof. (a) Send a given natural transformation

$$
\alpha: \operatorname{Hom}_{\mathcal{C}}(-, X) \longrightarrow F
$$

to $p_{\alpha}:=(\alpha(X))\left(1_{X}\right) \in F(X)$ where $1_{X}$ is the identity morphism of $X$. Conversely, given an element $p \in F(X)$, define the natural transformation $\alpha_{p}: \operatorname{Hom}_{\mathcal{C}}(-, X) \longrightarrow F$ by $\left(\alpha_{p}(Y)\right)(\phi)=(F(\phi))(p) \in$ $F(Y)$ for each $Y \in \mathcal{C}$ and $\phi \in \operatorname{Hom}_{\mathcal{C}}(Y, X)$. Then the associations $\alpha \mapsto p_{\alpha}$ and $p \mapsto \alpha_{p}$ are inverse to each other.

(b) For any object $X^{\prime}$ in $\mathcal{C}$, set $F=\operatorname{Hom}_{\mathcal{C}}\left(-, X^{\prime}\right)$ and apply part (a)

\section{QED}

4.4.1.5 Remark. From Yoneda's lemma we see that if a contravariant functor is represented by a pair as in (4.4.1.1), then the pair is determined uniquely up to a unique isomorphism.

4.4.1.6 Remark. We may also define a covariant functor $F: \mathcal{C} \longrightarrow \mathcal{S}$ to be representable if it is functorially isomorphic to $\operatorname{Hom}_{\mathcal{C}}(X,-)$ for some object $X$ in $\mathcal{C}$.

\subsubsection{Examples of Representable Functors}

\subsubsection{Example from Commutative Algebra: Existence of Universal Derivations}

Let $A, R$ denote commutative rings. Let $A$ be an $R$-algebra and $M$ an $A$ module. By a derivation of $A$ over $R$ we mean an $R$-linear map $D: A \longrightarrow M$ such that

$$
D(a b)=a \cdot D(b)+b \cdot D(a), \forall a, b \in A .
$$


Then $D(R)=0$ and the set of such derivations naturally forms an $A$-module denoted by $\operatorname{Der}_{R}(A, M)$. We thus get a covariant functor

$$
\operatorname{Der}_{R}(A,-): \operatorname{Mod}_{A} \longrightarrow \operatorname{Mod}_{A}
$$

Here $\operatorname{Mod}_{A}$ denotes the category of all $A$-modules. This functor is representable (see Lang [22], chap. XIX, sec. 3). Equivalently, there exists an $A$-module $\Omega$ and a universal derivation $d: A \longrightarrow \Omega$ such that for $D \in \operatorname{Der}_{R}(A, M)$, there exists a unique $A$-homomorphism $f: \Omega \longrightarrow M$ with $D=f \circ d$ and the association $D \mapsto f$ gives a functorial isomorphism

$$
\Phi: \operatorname{Der}_{R}(A,-) \stackrel{\sim}{\longrightarrow} \operatorname{Hom}_{A}(\Omega,-)
$$

In other words, the pair $(\Omega, \Phi)$ represents $\operatorname{Der}_{R}(A,-)$.

\subsubsection{Remarks: The Canonical Sheaf and Tangent Sheaf of a Compact Riemann Surface}

Let $X$ be a compact Riemann surface and let $T_{X}, T_{X}^{*}$ denote respectively its holomorphic tangent and cotangent (line) bundles. By the GAGA principle (A.10.5.1), since $X$ can be embedded as a closed submanifold of some complex projective space, there is a complex projective algebraic curve $\mathcal{C}$, and coherent locally free sheaves of $\mathcal{O}_{\mathcal{C}}$-modules $\mathcal{T}_{\mathcal{C}}$ (called the tangent sheaf) and $\omega_{\mathcal{C}}$ (called the canonical sheaf) such that, in the notations of (A.10.2) we have

$$
\mathcal{C}_{H O L}=X,\left(\mathcal{T}_{\mathcal{C}}\right)_{H O L}=\widetilde{T}_{X} \quad \text { and } \quad\left(\omega_{\mathcal{C}}\right)_{H O L}=\widetilde{T_{X}^{*}}
$$

where for a holomorphic vector bundle $V$ on $X, \tilde{V}$ denotes the associated sheaf over $X$ of germs of holomorphic sections of $V$.

Over any affine open subscheme $U=\operatorname{Spec}(A) \hookrightarrow \mathcal{C}$ (see (A.4.3) and (A.6.3)), consider the coherent sheaf $\Omega_{U / \mathbb{C}}$ over $U$ which is the sheaf associated to the module (in the sense of (A.6.9)) of universal derivations of $A$ over $\mathbb{C}$ as described above in (4.4.2.1). For various such affine opens $U_{i}$ which cover $\mathcal{C}$, the sheaves $\Omega_{U_{i} / \mathbb{C}}$ glue together to give a coherent locally free sheaf of rank one (invertible sheaf) which we denote by $\Omega_{\mathcal{C} / \mathbb{C}}$. Then we have

$$
\mathcal{T}_{\mathcal{C}}=\mathcal{H O} \mathcal{M}_{\mathcal{O}_{\mathcal{C}}}\left(\Omega_{\mathcal{C} / \mathbb{C}}, \mathcal{O}_{\mathcal{C}}\right) \quad \text { and } \quad \omega_{\mathcal{C}}=\Omega_{\mathcal{C} / \mathbb{C}}
$$

If $g$ is the genus of $X$ i.e., $X$ is topologically a sphere with $g$ handles attached, then we have that $g=\operatorname{dim}_{\mathbb{C}} \mathrm{H}^{0}\left(\mathcal{C}, \omega_{\mathcal{C}}\right)$. 


\subsubsection{Example from Algebraic Geometry: Existence of Dualizing Sheaves}

Let $k$ be any field. Let $X$ be a proper scheme over $\operatorname{Spec}(k)$ (i.e., the given morphism from $X$ to $\operatorname{Spec}(k)$ which makes $X$ a scheme over $k$ is a proper morphism (A.6.7)) of dimension $n$.

Let $X_{c}$ denote the category whose objects are coherent $\mathcal{O}_{X}$-modules (A.6.9) and whose morphisms are morphisms of $\mathcal{O}_{X}$-modules (A.3). Let $\mathrm{FDVS}_{c}$ denote the category whose objects are finite dimensional vector spaces over $k$ and whose morphisms are $k$-linear maps. Given $\mathcal{F}$ in $X_{c}$, we get the following contravariant representable functor

$$
\operatorname{Hom}_{X_{c}}(-, \mathcal{F}): X_{c} \longrightarrow \mathrm{FDVS}_{c} .
$$

Note also that the "top cohomology functor with values in a coherent $\mathcal{O}_{X^{-}}$ module" (see (A.8)) viz.

$$
\mathrm{H}^{n}(X,-): X_{c} \longrightarrow \mathrm{FDVS}_{c}
$$

is a covariant functor. Hence the functor

$$
\mathrm{H}^{n}(X,-)^{*}: X_{c} \longrightarrow \mathrm{FDVS}_{c},
$$

which assigns to each $\mathcal{F} \in X_{c}$, the dual of the $k$-vector space $\mathrm{H}^{n}(X, \mathcal{F})$, is a contravariant functor. A dualizing sheaf for $X$ is a coherent sheaf $\omega_{X}^{o} \in X_{c}$ which represents this contravariant functor. In other words we must have an isomorphism of functors

$$
\Phi: H^{n}(X,-)^{*} \stackrel{\sim}{\longrightarrow} \operatorname{Hom}_{X_{c}}\left(-, \omega_{X}^{o}\right)
$$

so that the pair $\left(\omega_{X}^{o}, \Phi\right)$ represents $\mathrm{H}^{n}(X,-)^{*}$. Grothendieck has shown that dualizing sheaves do exist.

\subsubsection{Remark: Serre Duality on a Compact Riemann Surface}

We continue with the notations of (4.4.2.2). The canonical sheaf $\omega_{\mathcal{C}}$ is a dualizing sheaf for $\mathcal{C}$. A related result is the Serre Duality Theorem which states that if $V$ is a holomorphic vector bundle on $X, V^{*}$ its dual, $\tilde{V}$ and $\widetilde{V^{*}}$ the corresponding sheaves over $X$ (of germs of holomorphic sections) and $\mathcal{K}=\Theta^{*}$, the sheaf associated to the holomorphic cotangent bundle of $X$, then we have canonical isomorphisms of $\mathbb{C}$-vector spaces

$$
\mathrm{H}^{1}(X, \tilde{V})^{*} \cong \mathrm{H}^{0}\left(X, \mathcal{K} \otimes \widetilde{V^{*}}\right)
$$

Of course, all the above statements can also be made in terms of $\mathcal{C}$ and algebraic vector bundles over $\mathcal{C}$. Note that by combining the above with the GAGA principle (A.10.5.1), we have the following expressions for the genus $g$ of $X($ or $\mathcal{C})$

$g=\operatorname{dim}_{\mathbb{C}}\left(\mathrm{H}^{0}\left(\mathcal{C}, \omega_{\mathcal{C}}\right)\right)=\operatorname{dim}_{\mathbb{C}}\left(\mathrm{H}^{1}\left(\mathcal{C}, \mathcal{O}_{\mathcal{C}}\right)\right)=\operatorname{dim}_{\mathbb{C}}\left(\mathrm{H}^{1}\left(X, \mathcal{O}_{X}\right)\right)=\operatorname{dim}_{\mathbb{C}}\left(\mathrm{H}^{0}(X, \mathcal{K})\right)$. 


\subsubsection{Example from Algebraic Topology: Classification of Principal G-Bundles}

Let $\mathcal{H}$ denote the category whose objects are topological spaces and whose morphisms are homotopy-equivalence classes of continuous maps. For an object $B \in \mathcal{H}$, we have the contravariant representable functor

$$
\operatorname{Hom}_{\mathcal{H}}(-, B): \mathcal{H} \longrightarrow \mathcal{S}
$$

where $\mathcal{S}$ denotes the category of sets and set-theoretic mappings.

Let $G$ be a topological group (e.g., a Lie group). For $X \in \mathcal{H}$, let $k_{G}(X)$ denote the set of isomorphism classes of numerable principal $G$-bundles over $X$. Since the pullbacks of a principal $G$-bundle by homotopic maps are isomorphic, we get a contravariant functor

$$
k_{G}(-): \mathcal{H} \longrightarrow \mathcal{S} .
$$

Next, let $\omega$ be a principal $G$-bundle over $B \in \mathcal{H}$. We define a natural transformation (morphism) of functors

$$
\Phi_{B, \omega}: \operatorname{Hom}_{\mathcal{H}}(-, B) \longrightarrow k_{G}(-)
$$

as follows:

$$
[f: X \rightarrow B] \in \operatorname{Hom}_{\mathcal{H}}(X, B) \mapsto\left[f^{*} \omega\right] \in k_{G}(X),
$$

where we use [-] to denote homotopy equivalence class or isomorphism class as relevant to the context of the usage.

It is a theorem of Milnor that $k_{G}(-)$ is representable. In fact, he constructs a topological space $B G$ (called the universal classifying space associated to the given $G$ ) and a principal $G$-bundle $\omega_{G}$ on $B G$ (called the universal principal $G$-bundle on $B G)$ such that the pair $\left(B G, \Phi_{B G, \omega_{G}}\right)$ represents $k_{G}(-)$.

\subsection{Functorial Definitions: Moduli Problems, Fine Moduli Spaces and Coarse Moduli Spaces}

\subsubsection{Moduli Problems}

4.5.1.1 Definition. Let the following data be given:

1. an object $X$ of a category $\mathcal{C}(\mathcal{C}$ satisfying (4.2.2));

2. a collection of objects $S$, belonging to a category whose objects and morphisms are defined intrinsically in terms of $X$; 
3. an equivalence relation $\sim_{S}$ on $S$;

4. a functor of families $\mathcal{F} \mathcal{A M}$ of objects of $S$ parametrized by elements of $\mathcal{C}$ as in (4.2.3).

The problem of (global) moduli of $S$ addresses the following questions:

1. to find an object $M$ in $\mathcal{C}$ such that the elements of the underlying set of $M$ are bijective in a canonical way to the elements of the set of equivalence classes $S / \sim_{S}$;

2. to investigate the ways in which the properties of families influence the structure of $M$.

Now, by Yoneda's lemma (4.4.1.4), we know that the structure on $M$ is uniquely determined by the representable functor $\operatorname{Hom}_{\mathcal{C}}(-, M)$. Hence we may investigate how the properties of the functor $\mathcal{F} \mathcal{A M}$ influence morphisms into $M$. More explicitly, we may pose the following questions:

1. given $Y \in \mathcal{C}, V \in \mathcal{F} \mathcal{A M}(M)$ and $\phi \in \operatorname{Hom}_{\mathcal{C}}(Y, M)$ we have the induced family $F=\phi^{*} V$ on $Y$. We may ask conversely if to each family $F$ on $Y$ there is associated a morphism $\phi_{F}: Y \longrightarrow M$ and a family $V_{F}$ on $M$ such that $\phi_{F}^{*}\left(V_{F}\right)=F$;

2. we could ask if there exists a single family $V$ on $M$ which affirmatively answers (1) above for every family $F$ on $Y$;

3. still better, we could ask if there exists a single family $V$ on $M$ which affirmatively answers (2) above for every $Y \in \mathcal{C}$.

If we are able to answer (3) of the above in the affirmative, then the families parametrized by each $Y \in \mathcal{C}$ determine all the morphisms $Y \longrightarrow M$, so that any known properties of the functor $\mathcal{F} \mathcal{A M}$ should possibly translate into properties of the functor $\operatorname{Hom}_{\mathcal{C}}(-, M)$ which determines $M$. This is what we mean when we require that the structure of an object of $\mathcal{C}$ on $S / \sim_{S}$ should relate naturally to and reflect properties of families of objects of $S$ parametrized by elements of $\mathcal{C}$

Since we do not want to distinguish between equivalent objects of $S$, we could have posed all the above questions in terms of the functor $\mathcal{F}$ of equivalence classes of families induced by the functor $\mathcal{F} \mathcal{A M}$ as explained in (4.2.5).

\subsubsection{Examples of Moduli Problems}

The examples of section 4.3 above, viz. (4.3.1) through (4.3.3), are all examples of moduli problems naturally occurring in Geometry and these may be called the fundamental nontrivial examples.

Note that in the latter two of these examples and in the first example with $M$ a compact projective manifold, the set $S$ is a collection of complex 
analytic objects each of which also has a unique algebraic structure due to the GAGA principle. Hence not only can we investigate if the elements of $S$ could be parametrized by a suitable complex analytic space but also it makes sense to ask if a parametrization could be given by a scheme over $\operatorname{Spec}(\mathbb{C})$. Of course, in the former case the families of objects of $S$ considered should be complex analytic families, whereas in the latter we must consider only algebraic families.

Corresponding to each of these cases, we thus obtain respectively a "complex analytic version" and an "algebraic version" of the problem of moduli of elements of $S$ modulo the equivalence $\sim_{S}$. The study of the relationships between the properties of these two moduli functors not only draws from the deep interplay of complex analytic geometry and algebraic geometry over an algebraically closed field of characteristic zero, but also enriches this interplay by providing new insight.

We shall completely characterize the complex analytic version of the moduli functor of example (4.3.2) for the case $n=1, d=0$ in section 4.6 following which, in section 4.7 we shall completely characterize both versions (complex analytic and algebraic) of the moduli functor of example (4.3.3).

\subsubsection{Fine Moduli Spaces}

4.5.3.1 Definition. Given a moduli problem as in (4.5.1) above, a fine moduli space is a pair $(M, \Phi)$ with $M \in \mathcal{C}$, which represents (in the sense of (4.4.1.1)) the functor $\mathcal{F}$ of equivalence classes of families. The pair $(M, \Phi)$ is determined uniquely up to a unique isomorphism due to Yoneda's lemma (4.4.1.4).

The existence of a fine moduli space is clearly the best solution that we can expect of a moduli problem, since in such a case all the questions posed in (4.5.1) are answered in the affirmative. We will see this in some detail below.

4.5.3.2 Remark. Let $(M, \Phi)$ be a fine moduli space. Then for each $T \in \mathcal{C}$, we have a bijection

$$
\Phi(T): \mathcal{F}(T) \stackrel{\cong}{\longrightarrow} \operatorname{Hom}_{\mathcal{C}}(T, M)
$$

making $\sim_{T}$-equivalence classes of families of objects of $S$ parametrized by $T$ to correspond uniquely to all the morphisms $T \longrightarrow M$.

Taking for $T$ a base-point-object $P$, we get a bijection of the set of equivalence classes of objects of $S$ with the underlying set of $M$. Hence we may transport any structure (e.g., topological, differentiable, complex analytic or algebraic) on $M$ to $S / \sim_{S}$. 
In order to answer how this structure on $S / \sim_{S}$ naturally reflects properties of families of objects of $S$ parametrized by objects of $\mathcal{C}$, just note that for any given family $V$ on $T \in \mathcal{C}$, the set-theoretic map

$$
|T| \longrightarrow S / \sim_{S}: t \mapsto\left[V_{t}\right],
$$

where $V_{t}$ is the fiber of $V$ over $t \in T$ (see (3) of (4.2.4)) and [-] denotes $\sim_{S}$-equivalence class, is actually a morphism from $T$ to $M$ and is in fact the unique morphism corresponding (via $\Phi(T)$ ) to the $\sim_{T}$-equivalence class of $V$ !

Notice that the bijection

$$
\Phi(M): \mathcal{F}(M) \stackrel{\cong}{\longrightarrow} \operatorname{Hom}_{\mathcal{C}}(M, M)
$$

defines a unique $\sim_{M}$-equivalence class of families parametrized by $M$ viz. $\Phi(M)^{-1}\left(1_{M}\right)$, where $1_{M}$ is the identity morphism of $M$ in $\mathcal{C}$. This will turn out to be a "universal family" which we formally define below.

\subsubsection{Universal Families}

4.5.3.3.1 Definition. Given a moduli problem as in (4.5.1) above, a universal family for this problem consists of a family $U=U(M)$, of objects of $S$ parametrized by an object $M$ of $\mathcal{C}$ called the (associated) universal classifying space, satisfying the following universal property: for every family $F$ parametrized by each $T \in \mathcal{C}$, there exists a unique morphism $\phi_{F}: T \longrightarrow$ $M$ such that the corresponding map due to the (contravariant!) functor $\mathcal{F}$ viz.

$$
\mathcal{F}\left(\phi_{F}\right): \mathcal{F}(M) \longrightarrow \mathcal{F}(T),
$$

maps the $\sim_{M}$-equivalence class of $U$ to the $\sim_{T}$-equivalence class of $F$.

In other words, every family arises as a pullback of $U$ by a unique morphism into $M$.

The connection between the concept of a universal family and a fine moduli space is summarized in the following.

4.5.3.3.2 Proposition. Let $(M, \Phi)$ be a fine moduli space. Then any family representing the $\sim_{M}$-equivalence class $\Phi(M)^{-1}\left(1_{M}\right)$ is a universal family and $M$ its associated universal classifying space. Conversely, if $U(N)$ is a universal family parametrized by its associated universal classifying space $N \in \mathcal{C}$, then the pair $(N, \Psi)$ is a fine moduli space, where for each $T \in \mathcal{C}$, we define

$$
\Psi(T): \mathcal{F}(T) \longrightarrow \operatorname{Hom}_{\mathcal{C}}(T, N),[F] \mapsto \phi_{F},
$$

where $\phi_{F}$ is obtained from the hypotheses on $U(N)$.

We omit the proof since it is a formal verification. 
4.5.3.3.3 Remarks. We can deduce the following two important properties of a fine moduli space from the discussion so far. We explicitly mention them here because their validity can be used in some instances to prove the existence of a fine moduli space (see (4.5.5)).

1. Let $U$ be a universal family on $M$. Then for each point $m \in M$, the fiber $U_{m}$ of $U$ over $m$ is in the same $\sim_{S}$-equivalence class as represented by $m$ itself.

2. If two families parametrized by a fixed object of $\mathcal{C}$ are fiberwise $\sim_{S}-$ equivalent, then they are themselves equivalent as families.

Thus we deduce that if a fine moduli space exists, then the equivalence relation on families is completely determined by the equivalence relation $\sim_{S}$ on objects of $S$.

Motivated by the previous proposition, we make the following alternative definition of fine moduli space.

4.5.3.3.4 Definition. Given a moduli problem as in (4.5.1), we may define a fine moduli space to be a pair $(U=U(M), M)$ consisting of a universal family $U$ on the associated universal classifying space $M \in \mathcal{C}$. It is clear that such a pair is uniquely determined up to a unique isomorphism due to the previous proposition and Yoneda's lemma (4.4.1.4).

4.5.3.3.5 Remarks. If one examines the proof of the previous proposition, which consists purely of simple category-theoretic verifications, one then sees that a contravariant functor $\mathcal{G}: \mathcal{A} \longrightarrow \mathcal{S}$ is represented by a pair $(M, \Phi), M \in \mathcal{A}$, if and only if the element $\Phi(M)^{-1}\left(1_{M}\right)$ of $\mathcal{G}(M)$ is a universal element in a sense similar to (4.5.3.3.1).

This universal element corresponds to $d: A \longrightarrow \Omega$ in (4.4.2.1); also the trace map of the Serre Duality Theorem given by the "sum of residue maps" in (4.4.2.3) and finally, in (4.4.2.5) it corresponds to the universal principal $G$-bundle $\omega_{G}$ on the universal classifying space $B G$.

\subsubsection{Coarse Moduli Spaces}

For many important moduli problems addressed in the context of categories such as those of complex analytic spaces, of schemes over $\mathbb{C}$, etc., it is observed that a fine moduli space does not exist except under additional restrictions.

It is clear that, for those moduli problems where there exist families exhibiting the jump phenomenon (see (2.8.2)), a fine moduli space - or even a coarse moduli space more generally - cannot exist even as a non-Haudorff topological space (or, in the context of the category of schemes, cannot exist even as a non-separated scheme.) 
We will explain later (in (4.8.2)) how one can construct "global obstructions" to the existence of a fine moduli space, when some of the objects to be parametrized have nontrivial automorphisms. Further, in many such cases, it can be shown that we cannot even obtain a tautological family - a notion weaker than that of a universal family — so that there cannot exist a universal family.

Another way to see why some moduli problems don't have a fine moduli space is by finding families that have all their fibers mutually equivalent, but which as families are nontrivial. This situation occurs, for example, in the problem of moduli of curves - see (4.8.2). But, as we saw in the previous subsection, the existence of a fine moduli space implies that the equivalence relation on families is completely determined by the equivalence relation on the objects to be parametrized.

However, it happens that in many moduli problems, we may obtain a "coarse moduli space" which still retains some of the good properties of a fine moduli space. We define this notion below. It must be noted that we may, in some situations, first prove that a moduli problem has a coarse moduli space and then construct a universal family to show that it is actually a fine moduli space. This is discussed in the next subsection and is exploited in (1) of (4.6.7.3).

4.5.4.1 Definition. Given a moduli problem as in (4.5.1), a coarse moduli space is a pair $(M, \Phi)$ where $M \in \mathcal{C}$ and

$$
\Phi: \mathcal{F} \longrightarrow \operatorname{Hom}_{\mathcal{C}}(-, M)
$$

is a natural transformation (i.e., a morphism of functors) such that the following hold:

1. for a base-point-object $P \in \mathcal{C}$, the mapping of sets $\Phi(P)$ is bijective;

2. for any object $N \in \mathcal{C}$ and any natural transformation

$$
\Psi: \mathcal{F} \longrightarrow \operatorname{Hom}_{\mathcal{C}}(-, N)
$$

there exists a unique natural transformation

$$
\Omega: \operatorname{Hom}_{\mathcal{C}}(-, M) \longrightarrow \operatorname{Hom}_{\mathcal{C}}(-, N)
$$

such that $\Psi=\Omega \circ \Phi$.

\subsubsection{Remarks.}

1. It trivially follows from the above definition that a fine moduli space is always a coarse moduli space. 
2. Condition (1) of the above definition implies that the set $S / \sim_{S}$ of equivalence classes of objects to be classified is identified by $\Phi(P)$ with the underlying set of $M$. This naturally defines on $S / \sim_{S}$ a structure making it an object of $\mathcal{C}$ (viz. $M$ ).

3. Let us discuss condition (2) of the above definition. Since $\Phi(P)$ is a bijection, we have that $\Omega(P)=\Psi(P) \circ \Phi(P)^{-1}$. By Yoneda's lemma (4.4.1.4), $\Omega$ corresponds uniquely to the morphism $(\Omega(M))\left(1_{M}\right) \in$ $\operatorname{Hom}_{\mathcal{C}}(M, N)$. Further, the underlying map of sets for this morphism is precisely $\Omega(P)$. Thus we can say that $\Omega(P)$ actually has the structure of a morphism in $\mathcal{C}$. This observation may be used to give an alternative definition of coarse moduli space (as we will see in proposition (4.5.4.5)). To discuss a more general observation, we first state the following.

4.5.4.3 Definition. Let $P$ be a base-point-object of $\mathcal{C}$. Given a bijective map of sets

$$
\alpha: S / \sim_{S}=\mathcal{F}(P) \stackrel{\cong}{\longrightarrow} \operatorname{Hom}_{\mathcal{C}}(P, M)=|M|,
$$

and given a family $F$ parametrized by $T \in \mathcal{C}$ we define the set-theoretic map

$$
\nu_{F}:|T|=\operatorname{Hom}_{\mathcal{C}}(P, T) \longrightarrow \operatorname{Hom}_{\mathcal{C}}(P, M)=|M|, h \mapsto \alpha\left(\left[h^{*} F\right]\right),
$$

where we note that $h^{*} F$ is the family on $P$ induced by $h$, hence an object of $S ;\left[h^{*} F\right]$ denotes its equivalence class and hence is an element of $\mathcal{F}(P)=$ $S / \sim_{S}$.

The connection of the above map $\nu_{F}$ to a coarse moduli space is explained in the following.

4.5.4.4 Proposition. Let $(M, \Phi)$ be a coarse moduli space. Take for $\alpha$ in the above definition, the bijection given by $\Phi(P)$. Then:

1. for any $T \in \mathcal{C}$ and any family $F$ parametrized by $T$, the set-theoretic mapping $\nu_{F}$ has the structure of a morphism of $\mathcal{C}$ i.e. it is the underlying map of the morphism $(\Phi(T))([F])$;

2. for any $T \in \mathcal{C}$ and any natural transformation

$$
\Psi: \mathcal{F} \longrightarrow \operatorname{Hom}_{\mathcal{C}}(-, T),
$$

the map of sets $\Psi(P) \circ \alpha^{-1}:|M| \longrightarrow|T|$ has the structure of the morphism $\Omega(P)$ in $\mathcal{C}$.

The proof is a straightforward formal verification and we omit it.

The above proposition suggests that the pair $(M, \alpha)$ may characterize $(M, \Phi)$. We have the following result in this direction. 
4.5.4.5 Proposition. Let $M \in \mathcal{C}$ be an object alongwith a bijective map of sets

$$
\alpha: S / \sim_{S}=\mathcal{F}(P) \stackrel{\cong}{\longrightarrow} \operatorname{Hom}_{\mathcal{C}}(P, M)=|M|
$$

such that the following hold:

1. for any $T \in \mathcal{C}$ and any family $F$ parametrized by $T, \nu_{F}$ has the structure of a morphism $\widetilde{\nu_{F}}: T \longrightarrow M$ in $\mathcal{C}$;

2. for any $T \in \mathcal{C}$ and any natural transformation

$$
\Psi: \mathcal{F} \longrightarrow \operatorname{Hom}_{\mathcal{C}}(-, T),
$$

the map of sets $\Psi(P) \circ \alpha^{-1}:|M| \longrightarrow|T|$ has the structure of a morphism $\widetilde{\Omega_{P}}: M \longrightarrow T$ in $\mathcal{C}$.

Then we have the following:

(i) the association

$$
\Phi: \mathcal{F} \longrightarrow \operatorname{Hom}_{\mathcal{C}}(-, M)
$$

defined for each $T \in \mathcal{C}$ by

$$
\Phi(T): \mathcal{F}(T) \longrightarrow \operatorname{Hom}_{\mathcal{C}}(T, M),[F] \mapsto \widetilde{\nu_{F}},
$$

where $\widetilde{\nu_{F}}$ is given by (1) above, is a natural transformation;

(ii) $(M, \Phi)$ is a coarse moduli space and $\alpha=\Phi(P)$.

The proof is a formal verification and so we omit it.

Motivated by the above proposition, we state the following.

\subsubsection{Remarks.}

1. We may define a coarse moduli space to be the pair $(M, \alpha=\Phi(P))$ equivalently instead of the pair $(M, \Phi)$.

2. As a result of the above proposition, it is easy to check that a coarse moduli space $(M, \Phi)$ is uniquely defined up to a unique isomorphism, provided it exists. For, let $(M, \Phi)$ and $\left(M^{\prime}, \Phi^{\prime}\right)$ be two coarse moduli spaces and let $(M, \alpha)$ and $\left(M^{\prime}, \alpha^{\prime}\right)$ be the corresponding pairs. By the above proposition, we see that the set-theoretic map $\alpha^{\prime} \circ \alpha^{-1}$ : $|M| \longrightarrow\left|M^{\prime}\right|$ has the structure of a morphism $f$ from $M$ to $M^{\prime}$ and it is easy to check by symmetry that this morphism is actually an isomorphism. Further, this is the unique isomorphism that satisfies $|f| \circ \alpha=\alpha^{\prime}$. In the next two sections, we give an example of a fine moduli space, and an example of a moduli problem that has no fine moduli space but does have a coarse moduli space, respectively.

Meanwhile, we note the following clear distinction between a fine moduli space and a coarse moduli space with respect to the equivalence relation on families. 
4.5.4.7 Remark. It follows from the above discussion that the existence of a coarse moduli space does not imply any specific restriction on the choice of equivalence relation on families. However in contrast, as noted in (4.5.3.3.3), the equivalence relation on families is determined by the equivalence relation on $S$ if a fine moduli space were to exist.

\subsubsection{When is a Coarse Moduli Space a Fine Moduli Space?}

We have the following proposition that answers the above question. At this point, also recall (4.5.3.3.3).

4.5.5.1 Proposition. Let $(M, \Phi)$ be a coarse moduli space. Then $(M, \Phi)$ is a fine moduli space if and only if the following conditions hold:

1. there exists a family $U$ parametrized by $M$ such that for each point $m \in|M|$, the fiber of $U$ over $m$ viz. $U_{m}$ has $\sim_{S}$-equivalence class $\Phi(P)^{-1}(m)$;

2. the equivalence relation $\sim_{S}$ completely determines the equivalence relation on families, in the sense that if two families parametrized by the same base are fiberwise equivalent, then they are themselves equivalent (as families).

Proof. It is easy to see that condition (1) is the same as requiring that $\Phi(T)$ is surjective for each $T \in \mathcal{C}$ and that condition (2) is the same as requiring that $\Phi(T)$ is injective for each $T \in \mathcal{C}$ QED

In the next section, we discuss an example of a fine moduli space.

\subsection{Example of a Fine Moduli Space: Holo- morphic Line Bundles and the Jacobian}

We discuss in this section the moduli problem of (holomorphic) line bundles on a compact Riemann surface which is a special case of (4.3.2).

We consider only line bundles of a certain kind viz. those of "degree zero". More generally, we will need the notion of the "degree" of any holomorphic vector bundle on a compact Riemann surface. The following preliminaries are toward the definition and properties of this notion. After these preliminaries, we give the precise formulation in the fourth subsection.

\subsubsection{The Picard Group of a Ringed Space}

Let $X$ be a ringed space i.e., a pair $\left(X, \mathcal{O}_{X}\right)$ as in (A.2). Let $\mathcal{F}$ be a sheaf of $\mathcal{O}_{X}$-modules as defined in (A.3). We say $\mathcal{F}$ is locally free if there exists 
an open cover $\left\{U_{i}\right\}$ of $X$ such that for each index $i$, the restriction $\left.\mathcal{F}\right|_{U_{i}}$ is isomorphic, as a sheaf of $\left.\mathcal{O}_{X}\right|_{U_{i}}$-modules, to a direct sum of copies of $\left.\mathcal{O}_{X}\right|_{U_{i}}$ :

$$
\left.\mathcal{F}\right|_{U_{i}} \cong \oplus_{\alpha \in S_{i}} \mathcal{O}_{\alpha}, \quad \mathcal{O}_{\alpha}:=\left.\mathcal{O}_{X}\right|_{U_{i}}\left(\alpha \in S_{i}\right) .
$$

We define the rank of $\mathcal{F}$ on $U_{i}$ to be the cardinality of the set $S_{i}$.

If the underlying topological space of $X$ is connected, then the rank of a locally free sheaf is the same on each open subset of $X$ and is hence called the rank of the locally free sheaf. A locally free sheaf of rank one is also called a invertible sheaf.

We have the following result whose proof is straightforward.

4.6.1.1 Proposition. Let $X$ be a ringed space. If $\mathcal{L}, \mathcal{M}$ are invertible sheaves on $X$, then so is $\mathcal{L} \otimes \mathcal{M}:=\mathcal{L} \otimes_{\mathcal{O}_{X}} \mathcal{M}$ (the sheaf associated to the presheaf on $X$ given by $U \mapsto \mathcal{L}(U) \otimes_{\mathcal{O}_{X}(U)} \mathcal{M}(U), U$ an open subset of $\left.X\right)$. Define $\mathcal{L}^{-1}=\mathcal{H} \mathcal{O} \mathcal{M}_{\mathcal{O}_{X}}\left(\mathcal{L}, \mathcal{O}_{X}\right)$ (the sheaf associated to the presheaf on $X$ given by $U \mapsto \operatorname{Hom}_{\mathcal{O}_{X} \mid U}\left(\left.\mathcal{L}\right|_{U},\left.\mathcal{O}_{X}\right|_{U}\right), U$ an open subset of $\left.X\right)$. Then we have that

$$
\mathcal{L}^{-1} \otimes \mathcal{L} \cong \mathcal{H} \mathcal{O M}_{\mathcal{O}_{X}}(\mathcal{L}, \mathcal{L}) \cong \mathcal{O}_{X}
$$

Motivated by the above proposition, we state the following.

4.6.1.2 Definition. Let $X$ be a ringed space. Let $\operatorname{Pic}\left(X, \mathcal{O}_{X}\right)$ or simply $\operatorname{Pic}(X)$ denote the set of isomorphism classes of invertible sheaves on $X$. Then under the operation $\otimes, \operatorname{Pic}(X)$ is a group, called the Picard Group of $X$.

Using Čech cohomology (A.9), one easily checks the following.

4.6.1.3 Theorem. Let $X$ be a ringed space. Then $\operatorname{Pic}(X) \cong H^{1}\left(X, \mathcal{O}_{X}^{*}\right)$ where $\mathcal{O}_{X}^{*}$ denotes the sheaf of abelian groups on $X$ whose sections over an open subset $U$ of $X$ are the units in the ring of sections of $\mathcal{O}_{X}$ over $U$, with multiplication as the (abelian) group operation.

In the next subsection, we describe the notion of "Chern class" of a line bundle on a compact Riemann surface.

\subsubsection{The Chern Class of a Line Bundle}

Let $X$ be a compact Riemann surface. Regard $X$ as a ringed space i.e., the pair $\left(X, \mathcal{C}_{X}\right)$ where $\mathcal{C}_{X}$ is the sheaf of germs of continuous complex-valued functions on $X$. Then we can prove the following.

4.6.2.1 Theorem. Given an element $l \in P i c\left(X, \mathcal{C}_{X}\right) \cong H^{1}\left(X, \mathcal{C}_{X}^{*}\right)$ represented with respect to an open covering $\left\{U_{i}\right\}$ of $X$ by the non-vanishing continuous complex-valued functions $g_{i j}$ on $U_{i} \cap U_{j}$, associate the isomorphism class of the topological line bundle (topological vector bundle of rank 1) 
on $X$ defined by taking $\left\{g_{i j}\right\}$ as the transition functions relative to the covering $\left\{U_{i}\right\}$. Then this association is an isomorphism.

Next we regard $X$ as the ringed space $\left(X, \mathcal{O}_{X}\right)$ where $\mathcal{O}_{X}$ is as usual the sheaf of germs of holomorphic functions on $X$. We then have the following analogue of the previous theorem.

4.6.2.2 Theorem. Given an element $l \in \operatorname{Pic}\left(X, \mathcal{O}_{X}\right) \cong H^{1}\left(X, \mathcal{O}_{X}^{*}\right)$ represented with respect to an open covering $\left\{U_{i}\right\}$ of $X$ by the non-vanishing holomorphic functions $g_{i j}$ on $U_{i} \cap U_{j}$, associate the isomorphism class of the holomorphic line bundle (holomorphic vector bundle of rank 1) on $X$ defined by taking $\left\{g_{i j}\right\}$ as the transition functions relative to the covering $\left\{U_{i}\right\}$. Then this association is an isomorphism.

Next we define Chern classes.

4.6.2.3 Definition. Starting with the morphism of short exact sequences of sheaves on $X$ given by

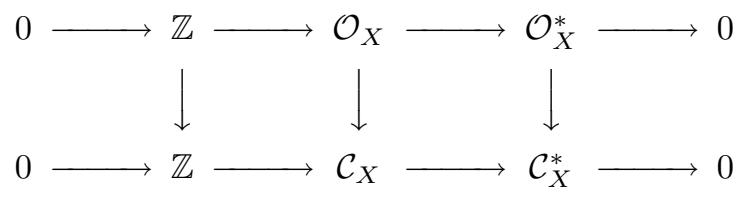

(where $\mathbb{Z}$ represents the constant sheaf on $X$, whose stalk at each point of $X$ is simply $\mathbb{Z}$ ), where the vertical arrows represent the obvious natural maps, the injections are the canonical inclusions and where the surjections are given by the exponential maps $f \mapsto e^{2 \pi i f}(i=\sqrt{-1})$, we get the following exact commutative diagram from the corresponding morphism of long exact sequences in cohomology (this follows from the fact that sheaf cohomology is a delta functor - see (A.8)):

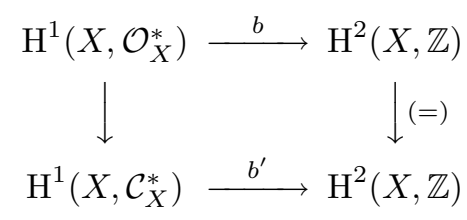

It can be checked that

$$
\mathrm{H}^{1}\left(X, \mathcal{C}_{X}\right)=\mathrm{H}^{2}\left(X, \mathcal{C}_{X}\right)=\mathrm{H}^{2}\left(X, \mathcal{O}_{X}\right)=0
$$

Thus $b$ is a surjection and $b^{\prime}$ is an isomorphism. Let $L$ be a topological (respectively holomorphic) line bundle on $X$ and let it define an element of $\mathrm{H}^{1}\left(X, \mathcal{C}_{X}^{*}\right)$ (respectively an element of $\mathrm{H}^{1}\left(X, \mathcal{O}_{X}^{*}\right)$ ) which we denote by $[L]$ (see (4.6.2.1) and (4.6.2.2)). Define for this line bundle its (first) topological 
Chern class (respectively (first) holomorphic Chern class) to be the element $c_{1}^{\prime}(L):=-b^{\prime}([L]) \in \mathrm{H}^{2}(X, \mathbb{Z})$ (respectively to be the element $c_{1}(L):=$ $\left.-b([L]) \in \mathrm{H}^{2}(X, \mathbb{Z})\right)$. Thus we have the following results.

\subsubsection{Theorem.}

1. Every topological line bundle on a compact Riemann surface can be endowed with the structure of a holomorphic line bundle.

2. Every topological line bundle on a compact Riemann surface is characterized (up to isomorphism) by its topological Chern class.

3. The underlying topological line bundle of a holomorphic line bundle on a compact Riemann surface is characterized (up to isomorphism) by its holomorphic Chern class.

In the next subsection, we define the "degree" of a holomorphic vector bundle on a compact Riemann surface.

\subsubsection{The Degree of a Vector Bundle}

Let $\mathcal{A}^{r}$ denote the sheaf of germs of $C^{\infty}$-differential $r$-forms on the underlying $C^{\infty}$-manifold of a compact Riemann surface $X$ for each $r, r=0,1,2$. Then we have the following exact sequence of sheaves on $X$ :

$$
0 \longrightarrow \mathbb{C} \longrightarrow \mathcal{A}^{0} \stackrel{d^{0}}{\longrightarrow} \mathcal{A}^{1} \stackrel{d^{1}}{\longrightarrow} \mathcal{A}^{2} \longrightarrow 0
$$

where $d^{i}: \mathcal{A}^{i} \longrightarrow \mathcal{A}^{i+1}$ is the exterior differential operator on $i$-forms for $i=0,1$. Here $\mathbb{C}$ denotes the constant sheaf on $X$ (with stalk $\mathbb{C}$ at each point of $X$ ) which is naturally a subsheaf of the sheaf $\mathcal{A}^{0}$ of complex-valued $C^{\infty}$-functions on $X$.

This exact sequence is called a resolution of the sheaf $\mathbb{C}$ and further has the following property: the cohomology groups $\mathrm{H}^{i}(X, \mathbb{C})$ can be calculated by applying the global-sections functor $\Gamma(X,-)$ (see $($ A.8)) to the sequence given by

$$
0 \longrightarrow \mathcal{A}^{0} \stackrel{d^{0}}{\longrightarrow} \mathcal{A}^{1} \stackrel{d^{1}}{\longrightarrow} \mathcal{A}^{2} \longrightarrow 0,
$$

and then by computing the cohomology of the resulting sequence. Thus we get the following de Rham isomorphism:

$$
\mathrm{H}^{2}(X, \mathbb{C})=\mathrm{H}^{0}\left(X, d^{1} \mathcal{A}^{1}\right) / d^{1} \mathrm{H}^{0}\left(X, \mathcal{A}^{1}\right) .
$$

Now given $v \in \mathrm{H}^{2}(X, \mathbb{C})$, choose (by the above isomorphism) a representative global 2 -form $\omega \in \mathrm{H}^{0}\left(X, d^{1} \mathcal{A}^{1}\right)$. Then the map

$$
I^{\prime}: \mathrm{H}^{2}(X, \mathbb{C}) \longrightarrow \mathbb{C}, v \mapsto \int_{X} \omega
$$


(where the integration is with respect to the canonical orientation on the underlying $C^{\infty}$-manifold of $X$ due to its complex manifold structure) is well-defined due to Stokes' Theorem, since

$$
\varphi \in d^{1} \mathrm{H}^{0}\left(X, \mathcal{A}^{1}\right) \Longrightarrow \int_{X} \varphi=0 .
$$

Recall that we have a canonical (actually injective) map $\mathrm{H}^{2}(X, \mathbb{Z}) \longrightarrow$ $\mathrm{H}^{2}(X, \mathbb{C})$ corresponding to the natural inclusion of constant sheaves $\mathbb{Z} \hookrightarrow \mathbb{C}$ on $X$. Let the composition of this map with $I^{\prime}$ be denoted $I: \mathrm{H}^{2}(X, \mathbb{Z}) \longrightarrow$ $\mathbb{C}$. We are now ready to state the following.

4.6.3.1 Definition. Let $L$ be a holomorphic line bundle on the compact Riemann surface $X$ and denote its isomorphism class by $[L] \in \mathrm{H}^{1}\left(X, \mathcal{O}_{X}^{*}\right)$. We define the degree of $L$ by the formula

$$
\operatorname{deg}(L):=I\left(c_{1}(L)\right)
$$

where $c_{1}(L)$ is the Chern class of $L$ as defined in (4.6.2.3) above.

We then have the following result which characterizes line bundles of degree zero.

\subsubsection{Theorem.}

1. I maps $H^{2}(X, \mathbb{Z})$ onto $\mathbb{Z} \subset \mathbb{C}$.

2. A holomorphic line bundle $L$ is of degree zero if and only if its first Chern class $c_{1}(L)=0$.

We next generalize the above notion of degree to holomorphic vector bundles of arbitrary rank - this will enable us to state the famous "Riemann-Roch" theorem, which we have already used in computations in (3.7.1) and (3.8.2).

4.6.3.3 Definition. Let $V$ be a holomorphic vector bundle of rank $n$ on the compact Riemann surface $X$. Define the determinant bundle of $V$ to be the $n$-th exterior power bundle of $V$ viz. $\Lambda^{n}(V)$ and denote it by Det $(V)$. This bundle is just the holomorphic line bundle on $X$ with transition functions given by the determinants of the matrices representing the transition functions of $V$ in $\mathbf{G L}(n, \mathbb{C})$ relative to a fixed open cover for $X$. We then define the degree of the vector bundle $V$ by the formula

$$
\operatorname{deg}(V):=\operatorname{deg}(\operatorname{Det}(V)) .
$$

This notion of degree has the following properties. 


\subsubsection{Lemma.}

1. If we have a short exact sequence of vector bundles on $X$ given by

$$
0 \longrightarrow V^{\prime} \longrightarrow V \longrightarrow V^{\prime \prime} \longrightarrow 0,
$$

then $\operatorname{deg}(V)=\operatorname{deg}\left(V^{\prime}\right)+\operatorname{deg}\left(V^{\prime \prime}\right)$.

2. If $V^{*}$ denotes the dual bundle of $V$, then $\operatorname{deg}\left(V^{*}\right)=-(\operatorname{deg}(V))$.

3. If $V_{1}$ and $V_{2}$ are vector bundles on $X$, then we have

$$
\operatorname{deg}\left(V_{1} \otimes V_{2}\right)=\operatorname{rank}\left(V_{1}\right) \cdot \operatorname{deg}\left(V_{2}\right)+\operatorname{rank}\left(V_{2}\right) \cdot \operatorname{deg}\left(V_{1}\right) .
$$

We now state the following nontrivial theorem, which is of fundamental use in numerical calculations in the Theory of Moduli. The theorem computes the degree of a vector bundle in terms of its rank, the Euler characteristic of the sheaf associated to this vector bundle and the Euler characteristic of the structure sheaf of $X$.

4.6.3.5 Riemann-Roch Theorem for Vector Bundles. Let $V$ be a holomorphic vector bundle of rank $n$ on a compact Riemann surface $X$ of genus $g$. Let $\tilde{V}$ denote the (coherent analytic locally free rank $n$ ) sheaf of germs of holomorphic sections associated to $V$. Then the degree of $V$ is given by the formula

$$
\operatorname{deg}(V)=\operatorname{dim}_{\mathbb{C}}\left(H^{0}(X, \tilde{V})\right)-\operatorname{dim}_{\mathbb{C}}\left(H^{1}(X, \tilde{V})\right)+n(g-1) .
$$

4.6.3.6 Remark. Let $\Theta$ denote the sheaf of germs of holomorphic vector fields on $X$ and let $W^{*}$ denote the dual of a holomorphic vector bundle $W$ on $X$. Since we have

$$
\mathrm{H}^{1}(X, \tilde{V})^{*} \cong \mathrm{H}^{0}\left(X, \mathcal{K} \otimes \widetilde{V^{*}}\right)
$$

by the Serre Duality Theorem stated in (4.4.2.4), we can rewrite the RiemannRoch formula as

$$
\operatorname{deg}(V)=\operatorname{dim}_{\mathbb{C}}\left(\mathrm{H}^{0}(X, \tilde{V})\right)-\operatorname{dim}_{\mathbb{C}}\left(\mathrm{H}^{0}\left(X, \Theta^{*} \otimes \widetilde{V^{*}}\right)\right)+n(g-1) .
$$

In the next subsection, we formulate the problem of moduli for line bundles of degree zero on a compact Riemann surface.

\subsubsection{The Moduli Problem for Degree Zero Line Bundles}

Let $X$ be a compact Riemann surface. We recall the moduli problem described in (4.3.2) for the particular case $n=1, d=0$. The corresponding 
functor $\mathcal{F}$ of equivalence classes of families will be denoted henceforth by $\mathrm{PIC}_{X}^{0}: \mathcal{C} \longrightarrow \mathcal{S}$ with notations as in (4.3.2).

Let $\mathcal{X}$ be the algebraic curve associated to $X$ (i.e., $\mathcal{X}_{H O L}=X$ in the notation of (A.10.1)) and recall that every holomorphic line bundle on $X$ has a unique structure of algebraic line bundle (which is a coherent locally free sheaf of rank 1) on $\mathcal{X}$ from (A.10.5.1).

In the above formulation of $\mathrm{PIC}_{X}^{0}$, if we replace $X$ by $\mathcal{X}$ and take for $\mathcal{C}$ the category of reduced and separated schemes over $\mathbb{C}$, then we get a contravariant moduli functor which we denote by $\mathrm{PIC}_{\mathcal{X}}^{0}: \mathcal{C} \longrightarrow \mathcal{S}$.

\subsubsection{Remarks.}

1. Note that both functors $\mathrm{PIC}_{X}^{0}$ and $\mathrm{PIC}_{\mathcal{X}}^{0}$ can be regarded as contravariant functors into the category of abelian groups and group homomorphisms.

2. Let us denote by $\mathcal{P}$ the category of projective reduced separated schemes over $\mathbb{C}$ and by $\mathcal{P}_{H O L}$ the category of reduced Hausdorff complex analytic spaces which can be embedded into some projective space $P_{\mathbb{C}}^{n}$ over $\mathbb{C}$ so that there is an equivalence of categories between $\mathcal{P}$ and $\mathcal{P}_{H O L}$ by the GAGA principle (A.10.5.1). Then the restrictions of the functors $\mathrm{PIC}_{X}^{0}$ and $\mathrm{PIC}_{\mathcal{X}}^{0}$ to $\mathcal{P}_{H O L}$ and $\mathcal{P}$ respectively can be identified.

\subsubsection{Construction of the Jacobian}

In this subsection, we construct using cohomology theory, the "Jacobian" of a compact Riemann surface which will later turn out to be a universal classifying fine moduli space for families of holomorphic line bundles of degree zero on the compact Riemann surface.

Let $X$ be a compact Riemann surface of genus $g$. Recall that $g=$ $\operatorname{dim}_{\mathbb{C}}\left(\mathrm{H}^{1}\left(X, \mathcal{O}_{X}\right)\right)=\operatorname{dim}_{\mathbb{C}}\left(\mathrm{H}^{0}\left(X, \Theta^{*}\right)\right)$.

It can be shown that if $\mathbb{R}$ denotes the constant sheaf of abelian groups with stalk $(\mathbb{R},+)$ at each point of $X$, then we have an isomorphism of the $\mathbb{R}$-vector space $\mathrm{H}^{1}(X, \mathbb{R})$ with the underlying $\mathbb{R}$-vector space of the complex vector space $\mathrm{H}^{1}\left(X, \mathcal{O}_{X}\right)$.

Next, the short exact sequence of sheaves on $X$ given by

$$
0 \longrightarrow \mathbb{Z} \longrightarrow \mathcal{O}_{X} \longrightarrow \mathcal{O}_{X}^{*} \longrightarrow 0
$$

described in (4.6.2.3) gives rise to a long exact sequence of cohomology groups (see (A.8)) from which we can extract the following short exact sequence of abelian groups

$$
0 \longrightarrow \mathrm{H}^{1}(X, \mathbb{Z}) \longrightarrow \mathrm{H}^{1}\left(X, \mathcal{O}_{X}\right) \longrightarrow \operatorname{PIC}_{X}^{0}(P) \longrightarrow 0,
$$


where $P$ denotes the degenerate one-point complex manifold and where we have used the facts that the canonical map $\mathrm{H}^{1}(X, \mathbb{Z}) \longrightarrow \mathrm{H}^{1}\left(X, \mathcal{O}_{X}\right)$ is injective and that $\operatorname{PIC}_{X}^{0}(P)$ is exactly the set, of isomorphism classes of holomorphic line bundles of degree zero on $X$, which is considered as an additive subgroup of the abelian group $\operatorname{Pic}\left(X, \mathcal{O}_{X}\right) \cong \mathrm{H}^{1}\left(X, \mathcal{O}_{X}^{*}\right)$ as in (4.6.1).

Now $\mathrm{H}^{1}(X, \mathbb{Z})$ is a lattice in the $2 g$-dimensional $\mathbb{R}$-vector space $\mathrm{H}^{1}(X, \mathbb{R})$. But, as we have stated above, this vector space is naturally isomorphic to the underlying real vector space of the complex vector space $\mathrm{H}^{1}\left(X, \mathcal{O}_{X}\right)$, so that we may consider $\mathrm{H}^{1}(X, \mathbb{Z})$ as a lattice in the complex vector space $\mathrm{H}^{1}\left(X, \mathcal{O}_{X}\right)$. The quotient of $\mathrm{H}^{1}\left(X, \mathcal{O}_{X}\right)$ by this lattice is a complex $g$ dimensional torus as explained in (1.5.2.2.1). But the above short exact sequence implies that, as abelian groups, we have the isomorphism $\mathrm{H}^{1}\left(X, \mathcal{O}_{X}\right) / \mathrm{H}^{1}(X, \mathbb{Z}) \cong \mathrm{PIC}_{X}^{0}(P)$. Therefore the abstract group $\mathrm{PIC}_{X}^{0}(P)$ can be given the structure of a complex $g$-dimensional torus such that the abelian group structure is induced from a complex Lie group structure. The resulting complex structure on $\mathrm{PIC}_{X}^{0}(P)$ is denoted by $\mathrm{Pic}^{0}(X)$.

It can be shown that the period matrix of the complex torus $\operatorname{Pic}^{0}(X)$ is a Riemann matrix and hence it is algebraizable by (2.7.1.1). The associated algebraic scheme over $\mathbb{C}$ has underlying set of closed points given by $\operatorname{PIC}_{X}^{0}(P)$ and is denoted by $\operatorname{Pic}^{0}(\mathcal{X})$ where $\mathcal{X}$ is the algebraic scheme associated to $X$.

In view of the above construction, we state the following.

4.6.5.1 Definition. We call $\operatorname{Pic}^{0}(X)$ as the Jacobian of the compact Riemann surface $X$ and denote it simply as $J$ or $J(X)$. Similarly, we call $\operatorname{Pic}^{0}(\mathcal{X})$ as the Jacobian of the curve $\mathcal{X}$ and denote it simply as $\mathcal{J}$ or $\mathcal{J}(\mathcal{X})$. The scheme $\mathcal{J}$ is called an abelian variety because it has a natural structure of commutative group scheme over $\mathbb{C}$ (the group axioms can be defined and verified entirely in terms of morphisms of schemes) and is an integral separated nonsingular scheme of finite type over $\mathbb{C}$ which is also proper over $\operatorname{Spec}(\mathbb{C})$.

4.6.5.2 Remark. When $X$ is an elliptic curve, it can be shown that $J(X)$ may be identified with $X$ and that $\mathcal{J}(\mathcal{X})$ may be identified with $\mathcal{X}$.

\subsubsection{Local Moduli for Degree Zero Line bundles and the Poincaré Family}

Using the Theories of Cohomology, Descent of Principal Bundles and Covering Spaces, one may construct the Poincaré Bundle $\mathbb{P}$ (a holomorphic line bundle) over $X \times J(X)$ for a compact Riemann surface $X$, where $J(X)$ is the Jacobian of $X$ as constructed in the previous subsection. We will 
later show that $\mathbb{P}$ is a universal family of holomorphic line bundles of degree zero on $X$ parametrized by $J(X)$. For the construction of $\mathbb{P}$, see M.S.Narasimhan's article [18]. We state the following results which are obtained as by-products of this construction.

4.6.6.1 Theorem. Let $X$ be a compact Riemann surface and $J:=J(X)$ be its Jacobian. Then there exists a holomorphic line bundle $\mathbb{P}=(\mathbb{P}, X \times$ $J, \pi)$ on the product complex manifold $X \times J$ called the Poincaré bundle which has the following properties:

1. for each holomorphic line bundle $L$ on $X$ of degree zero, if $j_{L} \in|J|$ is the corresponding point on $J$, then the fiber $\mathbb{P}_{j_{L}}$ of the family $\mathbb{P}$ over $j_{L}$ (i.e., the pullback of $\mathbb{P}$ to $X$ via the composition

$$
X \cong X \times P \stackrel{1_{X} \times \phi_{j_{L}}}{\hookrightarrow} X \times J
$$

where $P$ denotes the degenerate one-point complex manifold and the point $j_{L}$ of $J$ is canonically identified with the morphism $\phi_{j_{L}}: P \longrightarrow$ $J$ mapping onto $j_{L}$ ) as holomorphic line bundle on $X$ is of degree zero and is isomorphic to $L$ itself-in other words, the isomorphism class of $\mathbb{P}_{j_{L}}$ is $j_{L}$ itself;

2. for each point $j \in J$, the infinitesimal Kodaira-Spencer map for the family $\mathbb{P}$ of holomorphic line bundles on $X$ parametrized by $J$

$$
\eta_{j}: T_{j} J \longrightarrow H^{1}\left(X, \mathcal{E} \mathcal{N} \mathcal{D}\left(\mathbb{P}_{j}\right)\right)
$$

is an isomorphism.

\subsubsection{Remarks.}

1. Due to the See-Saw theorem (4.3.2.1), we see that $\mathbb{P}$ is uniquely determined, up to tensor product, with the pullback to $X \times J$ of some element of $\operatorname{Pic}(J)$.

We shall fix a Poincaré bundle $\mathbb{P}$ for the rest of this section.

2. For a holomorphic vector bundle $V$ on the compact Riemann surface $X$ we have the canonical isomorphism

$$
\mathcal{E N D}(V) \cong \widetilde{V \otimes V^{*}},
$$

where $\mathcal{E N D}$ denotes the sheaf (of germs of holomorphic sections) associated to the holomorphic vector bundle of endomorphisms of $V, V^{*}$ denotes the dual bundle of $V$ and $\widetilde{W}$ denotes the sheaf associated to a holomorphic vector bundle $W$ on $X$. In particular, if $L$ is a holomorphic line bundle on $X$, we have the canonical isomorphisms

$$
\mathcal{E} \mathcal{N} \mathcal{D}(L) \cong \widetilde{L \otimes L^{*}} \cong \tilde{L} \otimes \widetilde{L^{*}} \cong \tilde{L} \otimes(\tilde{L})^{-1} \cong \mathcal{O}_{X}
$$


since for $\tilde{L} \in(\operatorname{Pic}(X), \otimes)$ the inverse is precisely $\widetilde{L^{*}}$ (see (4.6.1.1)). Therefore we get the identification

$$
\mathrm{H}^{1}\left(X, \mathcal{E} \mathcal{N} \mathcal{D}\left(\mathbb{P}_{j}\right)\right) \stackrel{\cong}{\rightrightarrows} \mathrm{H}^{1}\left(X, \mathcal{O}_{X}\right) .
$$

Now, $T_{j} J$ and $\mathrm{H}^{1}\left(X, \mathcal{O}_{X}\right)$ are both complex vector spaces of the same dimension $g$. By explicit calculations we may check that $\eta_{j}$ is an injective map and hence conclude that this map is an isomorphism.

\subsubsection{Corollary (Local Moduli for Degree Zero Line Bundles).}

For each holomorphic line bundle $L$ of degree zero on a compact Riemann surface $X$, the germ of $J=J(X)$ at the point $j_{L}$ corresponding to $L$ is the local moduli space for $L$ and is smooth of dimension (equal to the dimension of $J$ which is) $g$ where $g$ is the genus of $X$. The Poincaré bundle $\mathbb{P}$ is a complete effectively and injectively parametrized family on $J$.

Completeness of $\mathbb{P}$ at each point of $J$ is proved by applying the analogue of the Theorem of Completeness (3.4.2.3), for families of holomorphic vector bundles, to the family $\mathbb{P}$ in view of (2) of the previous theorem.

Effectivity of $\mathbb{P}$ follows from the fact that the Kodaira-Spencer map is injective at each point of $J$ as guaranteed by (2) of the previous theorem.

$J$ is smooth by its very construction (see the previous subsection) and is of complex dimension $g$ as explained there.

Finally we note that the family $\mathbb{P}$ is injectively parametrized because of (1) of the previous theorem. Recall that these results were discussed more generally in (3.8.2).

\subsubsection{Fine Moduli for Line Bundles}

Recall the contravariant functor of equivalence classes of families of holomorphic line bundles of degree zero on a compact Riemann surface $X$ from (4.6.4) denoted by $\mathrm{PIC}_{X}^{0}: \mathcal{C} \longrightarrow \mathcal{S}$. We continue to use the notations of (4.6.4).

Recall that $|J|=\mathrm{H}^{1}\left(X, \mathcal{O}_{X}\right) / \mathrm{H}^{1}(X, \mathbb{Z})$ as abelian groups so that we have a natural bijection

$$
\alpha: \operatorname{PIC}_{X}^{0}(P) \stackrel{\cong}{\rightrightarrows} \operatorname{Hom}_{\mathcal{C}}(P, J)=|J|
$$

because of the short exact sequence

$$
0 \longrightarrow \mathrm{H}^{1}(X, \mathbb{Z}) \longrightarrow \mathrm{H}^{1}\left(X, \mathcal{O}_{X}\right) \longrightarrow \mathrm{PIC}_{X}^{0}(P) \longrightarrow 0
$$

as noted already in (4.6.5). Our first result is the following. 
4.6.7.1 Proposition. For any $T \in \mathcal{C}$ and any family $F$ of holomorphic line bundles of degree zero on $X$ parametrized by $T$, the set-theoretic map

$$
\nu_{F}:|T|=\operatorname{Hom}_{\mathcal{C}}(P, T) \longrightarrow \operatorname{Hom}_{\mathcal{C}}(P, J)=|J|, h \mapsto \alpha\left(\left[h^{*} F\right]\right)
$$

has the structure of a morphism $\widetilde{\nu_{F}}: T \longrightarrow J$ in $\mathcal{C}$.

Proof. For $t \in|T|$ let $F_{t}$ be the fiber of $F$ over $t$ (which is a holomorphic line bundle of degree zero on $X$ ) and let $j_{t}$ be its isomorphism class in $|J|$. Since the Poincaré bundle $\mathbb{P}$ is a complete family as indicated in the previous subsection, there exists an open neighborhood $U_{t}$ of $t$ in $T$ and a morphism in $\mathcal{C}$ :

$$
f_{t}: U_{t} \longrightarrow J, f_{t}(t)=j_{t},
$$

such that the pullback of $\mathbb{P}$ by $f_{t}$ is equivalent over $U_{t}$ to the restriction of $F$ to $U_{t}:\left.f_{t}^{*}(\mathbb{P}) \sim_{U_{t}} F\right|_{U_{t}}$ from which we get the following implications (using the See-Saw theorem (4.3.2.1))

$$
\left.f_{t}^{*}(\mathbb{P}) \cong F\right|_{U_{t}} \otimes p_{U_{t}}^{*}(L) \text { on } X \times U_{t} \Rightarrow \mathbb{P}_{f_{t}\left(t^{\prime}\right)} \cong F_{t^{\prime}} \text { for each } t^{\prime} \in U_{t},
$$

where $L$ is some holomorphic line bundle on $U_{t}$.

The map $\nu_{F}$ (introduced in (4.5.4.3)) in the present notation is simply given by

$$
\nu_{F}:|T| \longrightarrow|J|, t \mapsto j_{t} .
$$

Let $t^{\prime} \in U_{t}$ and $f_{t}\left(t^{\prime}\right)=j^{\prime}$. Then we get

$$
\mathbb{P}_{j^{\prime}} \cong F_{t^{\prime}} \Rightarrow\left[\mathbb{P}_{j^{\prime}}\right]=j_{t^{\prime}} \Rightarrow j^{\prime}=j_{t^{\prime}}
$$

by the property (1) of $\mathbb{P}$ mentioned in theorem (4.6.6.1). Thus we have $f_{t}\left(t^{\prime}\right)=j_{t^{\prime}}$. Hence the underlying set-theoretic map of $f_{t}$ coincides with the restriction of the map $\nu_{F}$ to $U_{t}$. But $t$ being arbitrary, we see that the morphisms $f_{t}$ patch up to give a unique global morphism $f_{F}: T \longrightarrow J$ whose underlying set-theoretic map is simply $\nu_{F}$ so that we can take $\widetilde{\nu_{F}}=f_{F}$.

Note that the objects of $\mathcal{C}$ are reduced and Hausdorff, which guarantees that $f_{F}$ is the unique morphism whose underlying set-theoretic map is $\nu_{F}$ QED

Next we define the association

$$
\Phi: \mathrm{PIC}_{X}^{0}(-) \longrightarrow \operatorname{Hom}_{\mathcal{C}}(-, J)
$$

for each object $T \in \mathcal{C}$ by the set-theoretic map

$$
\Phi(T): \operatorname{PIC}_{X}^{0}(T) \longrightarrow \operatorname{Hom}_{\mathcal{C}}(T, J)
$$


which sends the $\sim_{T}$-equivalence class $[F]$ of a family $F$ parametrized by $T$ to $\widetilde{\nu_{F}}$ :

$$
\Phi(T):[F] \mapsto \widetilde{\nu_{F}}
$$

This map is well-defined since $\widetilde{\nu_{F}}$ depends only on the equivalence class $[F]$ of $F$.

It is routine to check that the above association $\Phi$ is a contravariant functor.

For $g \in \operatorname{Hom}_{\mathcal{C}}(T, J)$, if we set $F:=g^{*}(\mathbb{P})$, then by the above proof we have that $\widetilde{\nu_{F}}=g$. Therefore we infer that $\Phi$ is surjective.

Next we prove that $\Phi$ is injective as well. To this end, let $F_{1}$ and $F_{2}$ be two families parametrized by $T \in \mathcal{C}$ such that

$$
\widetilde{\nu_{F_{1}}}=\widetilde{\nu_{F_{2}}} \Leftrightarrow \Phi(T)\left(\left[F_{1}\right]\right)=\Phi(T)\left(\left[F_{2}\right]\right) .
$$

Therefore, looking at the underlying set-theoretic maps, we get $\nu_{F_{1}}=$ $\left|\widetilde{\nu_{F_{1}}}\right|=\left|\widetilde{\nu_{F_{2}}}\right|=\nu_{F_{2}}$. But for $t \in|T|$, we have by the very definitions that

$$
\nu_{F_{1}}(t)=\left[\left(F_{1}\right)_{t}\right] \text { and } \nu_{F_{2}}(t)=\left[\left(F_{2}\right)_{t}\right]
$$

This implies that for each $t$ we must have $\left(F_{1}\right)_{t} \cong\left(F_{2}\right)_{t}$. Remembering that $F_{i}$ are by definition holomorphic line bundles on $X \times T$ and applying the See-Saw theorem (4.3.2.1), we get $F_{1} \sim_{T} F_{2} \Rightarrow\left[F_{1}\right]=\left[F_{2}\right]$ so that $\Phi(T)$ is indeed injective as claimed for each $T \in \mathcal{C}$.

To sum up all that we have proved in the above discussion, we state the following.

4.6.7.2 Theorem (Fine Moduli for Line Bundles). Let $X$ be a compact Riemann surface, $J=J(X)$ its Jacobian and $\mathbb{P}$ be a fixed Poincaré bundle on $X \times J$. Then the contravariant global moduli functor $P I C_{X}^{0}$ : $\mathcal{C} \longrightarrow \mathcal{S}$ is representable. The representing pair may be taken to be $(J, \Phi)$, where $\Phi$ is as defined above. Equivalently, the representing pair may also be taken to be $(J, \mathbb{P})$. Thus $J$ is a fine moduli space for holomorphic line bundles of degree zero on $X$ and $\mathbb{P}$ is the universal family for families of such line bundles.

Before we end this section, we note the following.

\subsubsection{Remarks.}

1. We could have proved the above theorem in the following way also. First of all, one verifies that the hypotheses of (4.5.4.5) are satisfied. Hence $(J, \Phi)$ is a coarse moduli space for the moduli problem associated to $\mathrm{PIC}_{X}^{0}$. Next, the hypothesis (1) of (4.5.5.1) is satisfied by $U:=\mathbb{P}$ due to assertion (1) of theorem (4.6.6.1). Finally, the 
hypothesis (2) of (4.5.5.1) is satisfied because of the See-Saw theorem (4.3.2.1). Hence one concludes from (4.5.5.1) that $(J, \Phi)$ is a fine moduli space with universal family $\mathbb{P}$.

2. The functor $\operatorname{PIC}_{\mathcal{X}}^{0}$ is also representable and the representing scheme turns out to be $\operatorname{Pic}^{0}(\mathcal{X})=\mathcal{J}=\mathcal{J}(\mathcal{X})$ (here $\mathcal{X}$ is the scheme associated to $X$ and $\operatorname{Pic}^{0}(\mathcal{X})$ is the scheme associated to $J=J(X)=$ $\operatorname{Pic}^{0}(X)$ with notations as in (4.6.5.1)). Just as we have obtained the global fine moduli space $J(X)$ by glueing local moduli spaces, the scheme $\mathcal{J}(\mathcal{X})$ can also be similarly obtained. The existence of an algebraic local moduli space for any algebraic line bundle on a complete scheme is guaranteed by Schlessinger's Theorem [20], and that such local moduli spaces indeed glue to give the fine moduli scheme $\mathcal{J}(\mathcal{X})$ can be deduced by applying Artin's results [30].

3. Algebraic Local Moduli Space for a Line Bundle of Degree Zero. Whenever an algebraic moduli functor is representable, such as $\mathrm{PIC}_{\mathcal{X}}^{0}$, then the algebraic local moduli space of an element of $\operatorname{PIC}_{\mathcal{X}}^{0}(P)$ (where $P=\operatorname{Spec}(\mathbb{C})$ ) turns out to be the affine scheme given by the completion of the local ring at the point of the representing classifying space. Hence, for $j \in \operatorname{PIC}_{\mathcal{X}}^{0}(P)=|\mathcal{J}|$, the algebraic local moduli space is $\operatorname{Spec}\left(\widehat{\mathcal{O}_{\mathcal{J}, j}}\right)$ (where $\hat{R}$ denotes the completion of a local ring $R)$. Now the natural question is: what is the relationship between the algebraic local moduli space and the complex analytic local moduli space? The answer to this is: $\widehat{\mathcal{O}_{\mathcal{J}, j}} \cong \widehat{\mathcal{O}_{J,{ }_{H O L}}}$.

In the next section, we discuss an example of a moduli problem which has a coarse moduli space for its solution but for which there cannot exist a fine moduli space.

\subsection{The Necessity of the Concept of a Coarse Moduli Space: The Example of Elliptic Curves}

We recall the problem of moduli of elliptic curves described in (4.3.3). We denote respectively by $\mathbb{M}_{1}^{H O L}$ and $\mathbb{M}_{1}^{A L G}$ the complex analytic and algebraic moduli functors of equivalence classes of families of elliptic curves. More precisely, $\mathbb{M}_{1}^{H O L}: \mathcal{H} \longrightarrow \mathcal{S}$ is the contravariant functor, from the category $\mathcal{H}$ of reduced Hausdorff complex analytic spaces, to the category $\mathcal{S}$ of sets, defined for each $T \in \mathcal{H}$ by

$\mathbb{M}_{1}^{H O L}(T)$

$=\{$ complex analytic families of elliptic curves parametrized by $T\} / \sim_{T}$, 
where $\sim_{T}$ denotes complex analytic equivalence of families parametrized by $T$ and $\mathbb{M}_{1}^{A L G}: \mathcal{A} \longrightarrow \mathcal{S}$ is the contravariant functor, from the category $\mathcal{A}$ of reduced separated schemes over $\operatorname{Spec}(\mathbb{C})$, to the category $\mathcal{S}$, defined for each $T \in \mathcal{A}$ by

$\mathbb{M}_{1}^{A L G}(T)=\{$ algebraic families of elliptic curves parametrized by $T\} / \sim_{T}$,

where $\sim_{T}$ denotes algebraic equivalence of families parametrized by $T$.

We shall construct a global coarse moduli space, for the problem of moduli of elliptic curves, by glueing local moduli spaces. This method is similar to that of the previous section, where we constructed a global fine moduli space for the problem of moduli of degree zero line bundles on a compact Riemann surface, by glueing local moduli spaces. The "glueing of local moduli spaces" is implicitly achieved when one uses the existence of local moduli spaces to prove that the corresponding moduli functor has a fine (or a coarse) moduli space for its solution.

To begin with, in the following, we recall the existence of local moduli for elliptic curves (already established in generality — for complex tori of arbitrary dimension — in (3.4.4)).

\subsubsection{Local Moduli for Elliptic Curves}

Consider the natural complex analytic family $(\mathcal{M}, U, \phi)$ of elliptic curves (complex tori of complex dimension 1) parametrized by the upper halfplane $U$ described in (2.4.2). For each $\tau \in U$, the fiber of this family $\mathcal{M}_{\tau}:=\phi^{-1}(\tau)$ is an elliptic curve $T(\tau)=\mathbb{C} / L(\tau)$ where $L=L(\tau)=$ $\{n+m \tau ; n, m \in \mathbb{Z}\}$ is the associated lattice.

At each point $\tau \in U$, the infinitesimal Kodaira-Spencer map for this family

$$
\rho_{\tau}: T_{\tau} U \longrightarrow \mathrm{H}^{1}\left(\mathcal{M}_{\tau}, \Theta_{\mathcal{M}_{\tau}}\right),
$$

is a nonzero map of complex vector spaces of dimension 1 and therefore it is an isomorphism. Hence using the Theorem of Completeness (3.4.2.3) we obtain the following.

4.7.1.1 Theorem (Local Moduli for Elliptic Curves). The natural complex analytic family $(\mathcal{M}, U, \phi)$ of elliptic curves parametrized by the upper half-plane $U$ is a complete effectively parametrized family. Further, for each elliptic curve $E$, if we choose $\tau \in U$ such that $\mathcal{M}_{\tau}:=\phi^{-1}(\tau)$ is isomorphic to $E$, then the germ of $U$ at $\tau$ is the local moduli space of $T$ and this germ is smooth and is of complex dimension 1.

We next define the "elliptic modular function" associated to a family of elliptic curves. 


\subsubsection{The Elliptic Modular Function $J_{F}$ Associated to a Family $F$}

Recall from (1.5.2.6.3) that for $(\mathcal{M}, U, \phi)=\mathcal{M} \in \mathbb{M}_{1}^{H O L}(U)$, we have the automorphic functions $\lambda: U \longrightarrow \mathbb{C}$ and $J: U \longrightarrow \mathbb{C}$. While $\lambda$ is invariant only under a proper subgroup of $\mathbf{P S L}(2, \mathbb{Z})$, recall that the elliptic modular function $J$ is $\mathbf{P S L}(2, \mathbb{Z})$-invariant. Recall further that both $\lambda$ and $J$ are holomorphic, that $\lambda$ never assumes the values $\{0,1\}$ and that $J$ is related to $\lambda$ by the formula

$$
J(\tau)=\frac{4}{27} \frac{\left(1-\lambda(\tau)+(\lambda(\tau))^{2}\right)^{3}}{(\lambda(\tau))^{2}(1-\lambda(\tau))^{2}}
$$

for each $\tau \in U$. Finally recall from (1.5.3.1) that since

$$
\mathcal{M}_{\tau} \cong \mathcal{M}_{\tau^{\prime}} \Leftrightarrow J(\tau)=J\left(\tau^{\prime}\right)
$$

we get a biholomorphic map $\tilde{J}$ from the Riemann surface $U / \mathbf{P S L}(2, \mathbb{Z})$ to C. Since the underlying set of this Riemann surface is naturally identified with the set of conformal equivalence classes of elliptic curves, for any elliptic curve $E$, the quantity $\tilde{J}([E])$ is a unique well-defined complex number called the j-invariant of $E$ which completely characterizes the conformal equivalence class $[E]$ of $E$.

We will henceforth denote $J$ by $J_{\mathcal{M}}$ and $\lambda$ by $\lambda_{\mathcal{M}}$. The reason for this notation will become clear from the following discussion.

Consider an arbitrary family $F \in \mathbb{M}_{1}^{H O L}(T)$, written more explicitly as $p: F \longrightarrow T$, where $T \in \mathcal{H}$. We define the set-theoretic map

$$
J_{F}:|T| \longrightarrow|\mathbb{C}|, \quad J_{F}(t)=\tilde{J}\left(\left[F_{t}\right]\right)=\tilde{J}\left(\left[p^{-1}(t)\right]\right),
$$

where for an elliptic curve $E,[E]$ denotes its isomorphism class.

Then our fundamental result about $J_{F}$ is the following.

4.7.2.1 Theorem. The set-theoretic map $J_{F}$ defined above has the structure of a morphism in $\mathcal{H}$ i.e., it has a unique structure of a global holomorphic function on $T$.

Notation. We will also denote (by abuse of notation) the corresponding holomorphic function by $J_{F}$.

Proof. Fix $t \in T$ and consider the elliptic curve $F_{t}$. Then there exists $\tau \in U$ such that $\mathcal{M}_{\tau} \cong F_{t}$ where $\mathcal{M} \in \mathbb{M}_{1}^{H O L}(U)$ is the natural family of elliptic curves parametrized by the upper half-plane $U$. By theorem (4.7.1.1), $\mathcal{M}$ is a complete complex analytic family of elliptic curves, hence there exists 
a neighborhood $U_{t}$ of $t \in T$, and a (not necessarily unique) morphism $f: U_{t} \longrightarrow U$ (in $\mathcal{H}$ ) with $f(t)=\tau$, such that the pullback family $f^{*} \mathcal{M}$ is equivalent to the restriction of $F$ to $U_{t}$ :

$$
\left.f^{*} M \sim_{U_{t}} F\right|_{U_{t}}
$$

Thus, for each $s \in U_{t}, F_{s}$ is biholomorphic to the elliptic curve $\mathcal{M}_{f(s)}$. Hence we have for $s \in U_{t}$ that $\left(\left.J_{F}\right|_{U_{t}}\right)(s)=\left(J_{\mathcal{M}} \circ f\right)(s)$ which implies that $J_{F}$ restricted to $U_{t}$ is a holomorphic function. Since our choice of $t \in T$ was arbitrary, $J_{F}$ is a global holomorphic function on $T$ QED

4.7.2.2 Remark. Continuing with the notations of the above proof, we see that we can locally define a holomorphic function

$$
\lambda_{\left(U_{t}, f\right)}: U_{t} \longrightarrow \mathbb{C}, s \mapsto\left(\lambda_{\mathcal{M}} \circ f\right)(s) .
$$

Since $f$ is not unique, the above definition depends on $f$ and hence we do not get a global holomorphic function on $T$ which generalizes the holomorphic function $\lambda_{\mathcal{M}}: U \longrightarrow \mathbb{C}$, unlike the case of the holomorphic function $J_{F}$ : $T \longrightarrow \mathbb{C}$ which does generalize $J_{\mathcal{M}}: U \longrightarrow \mathbb{C}$ as we proved above.

It is clear that the global holomorphic function $J_{F}$ restricted to $U_{t}$ is related to the locally defined function $\lambda_{\left(U_{t}, f\right)}$ by the formula

$$
J=\frac{4}{27} \frac{\left(1-\lambda_{\left(U_{t}, f\right)}+\left(\lambda_{\left(U_{t}, f\right)}\right)^{2}\right)^{3}}{\left(\lambda_{\left(U_{t}, f\right)}\right)^{2}\left(1-\lambda_{\left(U_{t}, f\right)}\right)^{2}},
$$

since a similar formula relates $\lambda_{\mathcal{M}}$ and $J_{\mathcal{M}}$.

Next consider the "algebraic version" $\mathbb{M}_{1}^{A L G}$ of the moduli functor $\mathbb{M}_{1}^{H O L}$. Let $F \in \mathbb{M}_{1}^{A L G}(T)$ be an algebraic family of elliptic curves (over $\operatorname{Spec}(\mathbb{C}))$ parametrized by $T \in \mathcal{A}$ where we recall that $\mathcal{A}$ denotes the category of reduced separated schemes over $\operatorname{Spec}(\mathbb{C})$. Further recall that if $P:=\operatorname{Spec}(\mathbb{C})$, then for each $T \in \mathcal{A},|T|=\operatorname{Hom}_{\mathcal{A}}(P, T)$ denotes the set of closed points of $T$. We may, as in the complex analytic case, define the set-theoretic map

$$
J_{F}:|T| \longrightarrow \mathbb{C}, t \mapsto \tilde{J}\left(\left[\left(F_{t}\right)_{H O L}\right]\right)
$$

for each closed point $t \in T$, where $\left(F_{t}\right)_{H O L}$ is the unique underlying compact Riemann surface of genus 1 associated to $F_{t}$ by the GAGA principle (A.10.5.1).

Note that $\mathbb{C}$ is precisely the set of closed points $\left|\mathbb{A}_{\mathbb{C}, x}^{1}\right|$ of the affine line (scheme) over $\mathbb{C}: \mathbb{A}_{(\mathbb{C}, x)}^{1}:=\operatorname{Spec}(\mathbb{C}[x])$.

It is natural to expect the following analogue of the previous theorem. For a proof, see e.g., the beautiful article of Mumford-Suominen [23], chap. 3 . 
4.7.2.3 Theorem. There exists a unique structure of morphism in $\mathcal{A}$ on the above map $J_{F}$ i.e., there is a global regular function $T \longrightarrow \mathbb{A}_{(\mathbb{C}, x)}^{1}:=$ $\operatorname{Spec}(\mathbb{C}[x])$ whose restriction to closed points of $T$ is precisely the settheoretic map $J_{F}$. Further, for each closed point $t \in T$, there exists a (Zariski) open neighborhood $U_{t}$ and a regular function on this neighborhood

$$
\lambda_{U_{t}}: U_{t} \longrightarrow \mathbb{A}_{\mathbb{C}, x}^{1}
$$

such that the restriction of the regular function (associated to $J_{F}$ ) to $U_{t}$ is related to $\lambda_{U_{t}}$ by the formula

$$
J=\frac{4}{27} \frac{\left(1-\lambda_{U_{t}}+\left(\lambda_{U_{t}}\right)^{2}\right)^{3}}{\left(\lambda_{U_{t}}\right)^{2}\left(1-\lambda_{U_{t}}\right)^{2}} .
$$

4.7.2.4 Notation. By abuse of notation, we will denote the global regular function corresponding to $J_{F}$ also by $J_{F}$ itself.

Motivated by the discussion above, we state the following.

4.7.2.5 Definition. Let $F$ be a complex analytic family (respectively an algebraic family) of elliptic curves parametrized by a reduced Hausdorff complex analytic space $T$ (respectively by a reduced separated scheme $T$ over $\mathbb{C}$ ) and $J_{F}$ be the associated global holomorphic function (respectively the associated global regular function) defined on $T$ as described in the above theorems of this subsection. We call $J_{F}$ the elliptic modular function associated to the family $F$.

\subsubsection{The Coarse Moduli Space for Elliptic Curves}

Let $\mathbb{P}_{\mathbb{C}}^{2}$ denote the projective scheme of dimension 2 over $\operatorname{Spec}(\mathbb{C})$ so that its associated complex analytic space is simply the 2-dimensional complex projective space $P_{\mathbb{C}}^{2}$ over $\mathbb{C}$ considered naturally as complex manifold: $\left(\mathbb{P}_{\mathbb{C}}^{2}\right)_{H O L}=P_{\mathbb{C}}^{2}($ see $($ A.6.7) $)$.

Let $\{X, Y, Z\}$ be a set of homogeneous coordinates on $\mathbb{P}_{\mathbb{C}}^{2}$ naturally giving rise to homogeneous coordinates on $P_{\mathbb{C}}^{2}$ which we also denote by $\{X, Y, Z\}$.

Let $\lambda \in \mathbb{C}-\{0,1\}$ and consider the reduced closed subscheme $E_{\lambda}$ of $\mathbb{P}_{\mathbb{C}}^{2}$ defined by the vanishing of the following homogeneous polynomial of degree 3:

$$
p_{\lambda}(X, Y, Z):=Y^{2} Z-X(X-Z)(X-\lambda Z) .
$$

Then it can be checked that $E_{\lambda}$ is irreducible, separated, nonsingular, of finite type and of dimension 1 over $\operatorname{Spec}(\mathbb{C})$. Further, the genus of this curve, defined to be the number of $\mathbb{C}$-linearly independent global algebraic differentials, may be computed to be equal to 1 . Thus $E_{\lambda}$ is an elliptic curve over $\operatorname{Spec}(\mathbb{C})$. 
Next consider the analytic subset of $P_{\mathbb{C}}^{2}$ defined by the vanishing of $p_{\lambda}(X, Y, Z)$ and give it the natural structure of reduced complex analytic subspace. This subspace is verified to be connected and smooth so that it is actually a closed submanifold of $P_{\mathbb{C}}^{2}$ of dimension 1 . Thus it is a compact Riemann surface embedded in $P_{\mathbb{C}}^{2}$. The genus of this compact Riemann surface is verified to be 1 , so it is actually an elliptic curve. It turns out that this elliptic curve is in fact isomorphic to $\left(E_{\lambda}\right)_{H O L}$. All this may be deduced at once from the results of (A.10), in particular from the GAGA principle.

Recall from (1.5.2.6) that associated to $\tau \in U$, where $U$ denotes the upper half-plane in $\mathbb{C}$, we have the lattice $L=L(\tau)=\{n+m \tau ; n, m \in \mathbb{Z}\}$ and the Weierstrass $\wp$-function satisfying the differential equation

$$
\left(\wp^{\prime}(z)\right)^{2}-4(\wp(z))^{3}+g_{2} \wp(z)+g_{3}=0,
$$

using which the elliptic curve $\mathbb{C} / L=\mathcal{M}_{\tau}$ is biholomorphically embedded onto the closed submanifold of $P_{\mathbb{C}}^{2}$ defined by the vanishing of the polynomial

$$
P\left(x_{0}, x_{1}, x_{2}\right)=x_{0} x_{1}^{2}-4 x_{2}^{3}+g_{2} x_{0}^{2} x_{2}+g_{3} x_{0}^{3},
$$

where the $x_{i}$ are the homogeneous coordinates. Then, as stated in (1.5.2.6.3), we have the $j$-invariant of this elliptic curve as

$$
J_{\mathcal{M}}(\tau)=\frac{g_{2}^{3}}{g_{2}^{3}-27 g_{3}^{2}} .
$$

Now, if we solve for $\mu \neq 0,1$ from the equations

$$
g_{2}=\frac{1}{3} \cdot(4)^{1 / 3} \cdot\left(\mu^{2}-\mu+1\right), g_{3}=\frac{1}{27} \cdot(\mu+1) \cdot\left(2 \mu^{2}-5 \mu+2\right),
$$

which are the relations arising from a linear change of coordinates that transforms $P\left(x_{0}, x_{1}, x_{2}\right)$ to $p_{\mu}(X, Y, Z)$ which defines the elliptic curve $E_{\mu}$, then for each such value of $\mu$, we clearly have $\left(E_{\mu}\right)_{H O L} \cong \mathcal{M}_{\tau}$.

It is verified that $\lambda_{\mathcal{M}}(\tau)$ satisfies the above equations in $\mu$.

Choosing $\tau \in U$ such that $\lambda_{\mathcal{M}}(\tau)=\lambda$, we get the j-invariant of $E_{\lambda}$ as

$$
\frac{4}{27} \frac{\left(1-\lambda+\lambda^{2}\right)^{3}}{(\lambda)^{2}(1-\lambda)^{2}}
$$

Hence we have proved the following.

4.7.3.1 Theorem. The value of the function

$$
j(\lambda)=\frac{4}{27} \frac{\left(1-\lambda+\lambda^{2}\right)^{3}}{(\lambda)^{2}(1-\lambda)^{2}},(\lambda \neq 0,1),
$$

characterizes the elliptic curve $E_{\lambda}$ over $\operatorname{Spec}(\mathbb{C})$ up to isomorphism. 
We continue with the notations introduced from the beginning of this section.

The elliptic modular function $\tilde{J}: U / \operatorname{PSL}(2, \mathbb{Z}) \stackrel{\cong}{\longrightarrow}$ gives rise to a set-theoretic bijection

$$
\alpha:=|\tilde{J}|: \mathbb{M}_{1}^{H O L}(P)=S / \sim_{S} \stackrel{\cong}{\longrightarrow} \operatorname{Hom}_{\mathcal{H}}(P, \mathbb{C})=|\mathbb{C}|,
$$

where $S / \sim_{S}$ is the set of isomorphism classes of elliptic curves and $P$ denotes the degenerate one-point manifold.

We use the same map $\alpha$ as above to get a set-theoretic bijection between $\mathbb{M}_{1}^{A L G}(P)=S / \sim_{S}$ and $\left|\mathbb{A}_{\mathbb{C}, x}^{1}\right|=\mathbb{C}$ where $P$ will always denote $\operatorname{Spec}(\mathbb{C})$ in the context of the functor $\mathbb{M}_{1}^{A L G}$.

With these notations, we state the following.

4.7.3.2 Proposition. For any $T \in \mathcal{H}$ (respectively $T \in \mathcal{A})$ and any family $F$ parametrized by $T$ i.e., $F \in \mathbb{M}_{1}^{H O L}(T)$ (respectively $F \in \mathbb{M}_{1}^{A L G}(T)$ ), the set-theoretic map $\nu_{F}:|T| \longrightarrow|\mathbb{C}|$ (respectively $\nu_{F}:|T| \longrightarrow\left|\mathbb{A}_{\mathbb{C}, x}^{1}\right|$ ) defined by $t \mapsto \alpha\left(\left[F_{t}\right]\right)$ has the structure of a morphism $T \longrightarrow \mathbb{C}$ in $\mathcal{H}$ (respectively the structure of a morphism $T \longrightarrow \mathbb{A}_{\mathbb{C}, x}^{1}$ in $\mathcal{A}$.)

Proof. From the results of the previous subsection, we see that $J_{F}$ is the unique structure of morphism on $\nu_{F}$ QED

Next define the associations

$$
J^{H O L}: \mathbb{M}_{1}^{H O L} \longrightarrow \operatorname{Hom}_{\mathcal{H}}(-, \mathbb{C}), J^{A L G}: \mathbb{M}_{1}^{A L G} \longrightarrow \operatorname{Hom}_{\mathcal{A}}\left(-, \mathbb{A}_{\mathbb{C}, x}^{1}\right)
$$

for each $T \in \mathcal{H}$ and each $T^{\prime} \in \mathcal{A}$ by the set-theoretic maps

$$
\begin{aligned}
& J^{H O L}(T): \mathbb{M}_{1}^{H O L}(T) \longrightarrow \operatorname{Hom}_{\mathcal{H}}(T, \mathbb{C}), \\
& J^{A L G}\left(T^{\prime}\right): \mathbb{M}_{1}^{A L G}\left(T^{\prime}\right) \longrightarrow \operatorname{Hom}_{\mathcal{A}}\left(T^{\prime}, \mathbb{A}_{\mathbb{C}, x}^{1}\right)
\end{aligned}
$$

which are in turn defined by

$$
F \mapsto J_{F}, \quad \text { and } \quad F^{\prime} \mapsto J_{F^{\prime}}
$$

Then in view of the previous proposition, it is easy to prove the following.

4.7.3.3 Proposition. The associations $J^{H O L}, J^{A L G}$ are natural transformations i.e., morphisms of functors.

We will show that $\left(\mathbb{C}, J^{H O L}\right)$ and $\left(\mathbb{A}_{\mathbb{C}, x}^{1}, J^{A L G}\right)$ are coarse moduli spaces for $\mathbb{M}_{1}^{H O L}$ and $\mathbb{M}_{1}^{A L G}$ respectively. Now in view of (4.7.3.2) and (4.5.4.5), it is enough to check the hypothesis (2) of (4.5.4.5). 
To the above end, let $R:=\mathbb{C}\left[x,(x(1-x))^{-1}\right]$ be the localisation of the polynomial ring $\mathbb{C}[x]$ (in the variable $x$ over $\mathbb{C}$ ) with respect to the multiplicative subset $\left\{1, f, f^{2}, \ldots,\right\}$ with $f=x(1-x)$.

Then $C:=\operatorname{Spec}(R)$ is an affine scheme of finite type over $\mathbb{C}$ (see (A.4.3) and (A.6.3)) whose associated complex analytic space in the sense of (A.10.1) is just the complement of $\{0,1\}$ in the complex plane: $C_{H O L}=$ $\mathbb{C}-\{0,1\}$. Note that $C \in \mathcal{A}, C_{H O L} \in \mathcal{H}$.

We will "put together" the elliptic curves $E_{\lambda},(\lambda \neq 0,1)$ (respectively $\left.\left(E_{\lambda}\right)_{H O L}\right)$ to obtain a family over $C$ (respectively over $C_{H O L}$ ) with parameter $\lambda$.

Consider the reduced closed subscheme $E$ of $\mathbb{P}_{\mathbb{C}}^{2} \times C$ defined by the vanishing of the following polynomial which is homogeneous of degree 3 in $X, Y, Z$ and is of degree 1 in $x$ :

$$
p(X, Y, Z, x):=Y^{2} Z-X(X-Z)(X-x Z) .
$$

The canonical projection onto the second factor: $\mathbb{P}_{\mathbb{C}}^{2} \times C \longrightarrow C$ induces a projection $p: E \longrightarrow C$.

The $E$ constructed above is checked to be a separated scheme of finite type over $\mathbb{C}$ so that $E \in \mathcal{A}$.

Further, for each closed point $\lambda \in C$, the fiber of $p$ over $\lambda$ is simply the elliptic curve $E_{\lambda}$ described in the beginning of this subsection.

It is checked that $p$ is proper (say because projective spaces are complete) and is also smooth (or flat).

To sum up, $p: E \longrightarrow C$ is an element of $\mathbb{M}_{1}^{A L G}(C)$.

Using the results of (A.10), we see that $p_{H O L}: E_{H O L} \longrightarrow C_{H O L}=$ $\mathbb{C}-\{0,1\}$ is an element of $\mathbb{M}_{1}^{H O L}\left(C_{H O L}\right)$.

It is clear from the above discussion that the elliptic modular function associated to this family viz. $J_{E_{H O L}}: C_{H O L} \longrightarrow \mathbb{C}$ is precisely the holomorphic function $j: C_{H O L} \longrightarrow \mathbb{C}$ defined earlier.

Note that $j$ itself is the underlying morphism of complex analytic spaces associated to a morphism of affine schemes of finite type over $\mathbb{C}$ (which we will also denote by $j$ ) given by

$$
j: C=\operatorname{Spec}\left(\mathbb{C}\left[x,(x(1-x))^{-1}\right]\right) \longrightarrow \operatorname{Spec}(\mathbb{C}[x])=\mathbb{A}_{\mathbb{C}, x}^{1}
$$

canonically corresponding to the $\mathbb{C}$-algebra homomorphism

$$
\mathbb{C}[x] \longrightarrow \mathbb{C}\left[x,(x(1-x))^{-1}\right], x \mapsto j(x)
$$

where $j$ is the rational function defined in theorem (4.7.3.1).

With this (abuse of) notation we denote by $j$ the elliptic modular function associated to $E$ as well as $E_{H O L}$. We are now ready to prove the following key result. 
4.7.3.4 Theorem. Let $T \in \mathcal{H}$ and $T^{\prime} \in \mathcal{A}$ be given respectively alongwith natural transformations

$$
\Psi^{H O L}: \mathbb{M}_{1}^{H O L} \longrightarrow \operatorname{Hom}_{\mathcal{H}}(-, T), \quad \Psi^{A L G}: \mathbb{M}_{1}^{A L G} \longrightarrow H_{\mathcal{A}}\left(-, T^{\prime}\right) .
$$

Then each of the following natural set-theoretic maps

$$
\Psi^{H O L}(P) \circ \alpha^{-1}:|\mathbb{C}| \longrightarrow|T| \quad \text { and } \Psi^{A L G}(P) \circ \alpha^{-1}:\left|\mathbb{A}_{\mathbb{C}, x}^{1}\right| \longrightarrow\left|T^{\prime}\right|
$$

has a unique structure of morphism.

Proof. Since $\Psi^{A L G}$ and $\Psi^{H O L}$ are morphisms of functors, the families $E$ and $E_{H O L}$ with elliptic modular function $j$ give rise to the morphisms

$g^{\prime}:=\Psi^{A L G}(C)([E]): C \longrightarrow T^{\prime}$ and $g:=\Psi^{H O L}\left(C_{H O L}\right)\left(\left[E_{H O L}\right]\right): C_{H O L} \longrightarrow T$ respectively.

Consider the morphism $\left(j, g^{\prime}\right): C \longrightarrow \mathbb{A}_{\mathbb{C}, x}^{1} \times T^{\prime}$ (respectively the morphism $\left.(j, g): C_{H O L} \longrightarrow \mathbb{C} \times T\right)$. From the formula defining $j$ one deduces easily that $j$ is surjective and that $\left(j, g^{\prime}\right.$ ) (respectively $(j, g)$ ) is proper. Now since the image of a proper map is closed, we have that the set $\Gamma^{\prime}:=\operatorname{Image}\left(j, g^{\prime}\right)$ (respectively the set $\left.\Gamma:=\operatorname{Image}(j, g)\right)$ is closed and irreducible in $\mathbb{A}_{\mathbb{C}, x}^{1} \times T^{\prime}$ (respectively is closed and connected in $\mathbb{C} \times T$ ).

A simple computation shows that the morphism $p^{\prime}: \Gamma^{\prime} \longrightarrow \mathbb{A}_{\mathbb{C}, x}^{1}$ (respectively the morphism $p: \Gamma \longrightarrow \mathbb{C}$ ) induced by the canonical projection onto the first factor $\mathbb{A}_{\mathbb{C}, x}^{1} \times T^{\prime} \longrightarrow \mathbb{A}_{\mathbb{C}, x}^{1}$ (respectively by $\mathbb{C} \times T \longrightarrow \mathbb{C}$ ) is injective.

Since $j$ is surjective, so are $p$ and $p^{\prime}$.

Now by the famous Zariski's Main Theorem both $p$ and $p^{\prime}$ are isomorphisms.

Finally define the morphisms

$$
f^{\prime}:=p_{2}^{\prime} \circ\left(p^{\prime}\right)^{-1}: \mathbb{A}_{\mathbb{C}, x}^{1} \longrightarrow T^{\prime} \text { and } f:=p_{2} \circ(p)^{-1}: \mathbb{C} \longrightarrow T
$$

where $p_{2}^{\prime}$ (respectively $p_{2}$ ) is induced by the canonical projection onto the second factor from $\mathbb{A}_{\mathbb{C}, x}^{1} \times T^{\prime}$ (respectively by the canonical projection onto the second factor from $\mathbb{C} \times T)$. We then have $f^{\prime} \circ j=g^{\prime}, f \circ j=g$.

Remembering that $\Psi^{A L G}$ and $\Psi^{H O L}$ are morphisms of functors, a computation reveals that the underlying set-theoretic map of $f^{\prime}$ (respectively of $f$ ) is precisely $\Psi^{A L G}(P) \circ \alpha^{-1}$ (respectively $\Psi^{H O L}(P) \circ \alpha^{-1}$ ) QED

Using the conclusions of proposition (4.7.3.2) and the above theorem as hypotheses and applying (4.5.4.5), we obtain the following main result of this section.

4.7.3.5 Theorem (Coarse Moduli for Elliptic Curves). The pair $\left(\mathbb{C}, J^{H O L}\right)$ (respectively $\left.\left(\mathbb{A}_{\mathbb{C}, x}^{1}, J^{A L G}\right)\right)$ is a coarse moduli space for the problem of moduli of elliptic curves associated to the moduli functor $\mathbb{M}_{1}^{H O L}$ (respectively $\mathbb{M}_{1}^{A L G}$ ). 
4.7.3.6 Remark (Algebraic Local Moduli Space for an Elliptic Curve). The algebraic local moduli space for an elliptic curve with invariant $j$ is $\operatorname{Spec}\left(\widehat{\mathcal{O}_{Y, j}}\right)$ where $Y=\mathbb{A}_{\mathbb{C}, x}^{1}$ and we have the isomorphism of rings $\widehat{\mathcal{O}_{Y, j}} \cong \widehat{\mathcal{O}_{\mathbb{C}, j}}($ see $(3)$ of $(4.6 .7 .3))$.

The main aspect in which the problem of moduli of elliptic curves differs from that of moduli of line bundles considered in the previous section is that one cannot obtain a fine moduli space for the former. We shall highlight the reasons that imply the non-existence of a fine moduli space for elliptic curves in the next section.

\subsection{The Non-existence of a Fine Moduli Space for Elliptic Curves}

\subsubsection{Local Obstructions to Existence of a Tautological Family}

Given a moduli functor (of equivalence classes of families) as in (4.5.1), we have seen that the requirement that there exists a fine moduli space, for the moduli problem associated to this functor, is equivalent to the existence of a universal family (cf. (4.5.3.3.2)). We will show in this subsection how such a universal family cannot exist for the problem of moduli of elliptic curves. We shall in fact show in this case that there does not even exist a "tautological family" - a notion weaker than that of universal family which we define below.

\subsubsection{Tautological Families}

Let $\mathcal{F} \mathcal{A M}: \mathcal{C} \longrightarrow \mathcal{S}$ be a functor of families associated to a moduli problem as in (4.5.1) and let $\mathcal{F}: \mathcal{C} \longrightarrow \mathcal{S}$ be the functor of equivalence classes of families naturally arising from $\mathcal{F} \mathcal{A M}$ (see (4.2.5)).

4.8.1.1.1 Definition. A pair $(F, \beta)$ where $F \in \mathcal{F} \mathcal{A M}(T)$ for some object $T \in \mathcal{C}$ and where $\beta$ is a set-theoretic mapping of $S / \sim_{S}$ onto $|T|$ is called a tautological family for the moduli functor $\mathcal{F}: \mathcal{C} \longrightarrow \mathcal{S}$ if the following conditions are satisfied:

1. The map $\beta$ is a bijection of sets

$$
\beta: \mathcal{F}(P)=S / \sim_{S} \stackrel{\cong}{\longrightarrow} \operatorname{Hom}_{\mathcal{C}}(P, T)=|T|,
$$

which identifies $\sim_{S}$-equivalence classes of objects of $S$ with the underlying points of $T$. Here, as usual, $P$ denotes a base-point-object of $\mathcal{C}$ (see (4.2.2)); 
2. For each point $t \in|T|$ (thought of as a morphism $\phi_{t} \in \operatorname{Hom}_{\mathcal{C}}(P, T)$ ), the $\sim_{S}$-equivalence class of the fiber $F_{t}$ of $F$ at $t$ (viz. $\left[\phi_{t}^{*}(F)\right] \in$ $\left.\mathcal{F}(P)=S / \sim_{S}\right)$ is equal to that determined by $t$ via $\beta$ i.e., $\beta^{-1}(t)$.

4.8.1.1.2 Example. Considering the problem of moduli of degree zero line bundles on a compact Riemann surface $X$, we see from assertion (1) of (4.6.6.1) that the Poincaré bundle $\mathbb{P}$ is a tautological family.

More generally, every universal family is indeed a tautological family (cf. (1) of (4.5.3.3.3)).

It's clear that the non-existence of a tautological family implies the nonexistence of a universal family (or what is the same, of a fine moduli space) - this is how we show below that the problem of moduli of elliptic curves does not have a fine moduli space for its solution.

By analyzing various examples from the Theory of Moduli, it is known that whenever a set of objects in Geometry has to be classified (modulo isomorphism or some suitable equivalence relation), then one can never hope to obtain a fine moduli space (i.e., a universal family) if there exist objects in this set that admit nontrivial automorphisms. We shall explain later the meaning of this statement, in the context of the moduli problem of elliptic curves. To that end, we first describe below the group of automorphisms of an arbitrary elliptic curve.

\subsubsection{The Group of Automorphisms of an Elliptic Curve}

We state the following theorem, whose proof is nontrivial - see [23], chap. 3, or Hartshorne's book Algebraic Geometry, chap. IV, sec. 4, listed under the References at the end. Using the GAGA principle (A.10.5.1), we see that the group of automorphisms of the elliptic curve is the same irrespective of it being considered as a compact Riemann surface of genus 1 or as a projective nonsingular integral curve over $\operatorname{Spec}(\mathbb{C})$ of genus 1 .

4.8.1.2.1 Theorem. Let $E$ be an elliptic curve and let $[E]$ denote its isomorphism class, considered as an element of $S=\mathbb{M}_{1}^{H O L}(P)=\mathbb{M}_{1}^{A L G}(P)$ where $P$ is a degenerate smooth one-point-space. Let $\tilde{J}: U / \mathbf{P S L}(2, \mathbb{Z}) \stackrel{\cong}{\longrightarrow}$ be the (holomorphic) elliptic modular function and $\alpha: S \stackrel{\cong}{\longrightarrow}$ be the settheoretic bijection underlying this function. Then the group of automorphisms $G(E)$ of the elliptic curve $E$ is given up to isomorphism as additive abelian group by:

$$
G(E) \cong \begin{cases}\mathbb{Z} / 2 \mathbb{Z} & \text { if } \alpha([E]) \neq 0,1 \\ \mathbb{Z} / 6 \mathbb{Z} & \text { if } \alpha([E])=0 \\ \mathbb{Z} / 4 \mathbb{Z} & \text { if } \alpha([E])=1\end{cases}
$$


Thus, the two elliptic curves $E$ characterised by $j$-invariants $\alpha([E])=0$ and 1 respectively are precisely those admitting extra automorphisms. More explicitly, on the elliptic curve $E_{(-1)}$ given by the equation $Y^{2} Z=X^{3}-$ $X Z^{2}$ with $j$-invariant 1 we have the automorphism of order 4 given by $X \mapsto-X, Y \mapsto(\sqrt{-1}) Y$ and on $E_{(-\omega)}$ with normalized equation $Y^{2} Z=$ $X^{3}-Z^{3}$ of $j$-invariant 0 there is the automorphism of order 6 given by $X \mapsto \omega X, Y \mapsto-Y$ where $\omega$ denotes a nonreal cube root of unity.

\subsubsection{Local Obstruction to the Existence of a Tautological Family on $\mathbb{C}$}

We have shown in the previous subsection (see theorem (4.7.3.5)) that $\left(\mathbb{C}, J^{H O L}\right)$ (respectively $\left.\left(\mathbb{A}_{\mathbb{C}, x}^{1}, J^{A L G}\right)\right)$ is a coarse moduli space for the functor $\mathbb{M}_{1}^{H O L}$ (respectively $\mathbb{M}_{1}^{A L G}$ ). One naturally asks if these coarse moduli spaces have the additional property of being fine moduli spaces. This is the same as asking if there exist universal families. Since the notion of a universal family is stronger than that of a tautological family, one first therefore asks if there exists a tautological family on $\mathbb{C}$ (respectively on $\mathbb{A}_{\mathbb{C}, x}^{1}$ ). We shall show below that no such tautological family can exist, so that $\left(\mathbb{C}, J^{H O L}\right)$ and $\left(\mathbb{A}_{\mathbb{C}, x}^{1}, J^{A L G}\right)$ cannot be fine moduli spaces.

We shall arrive at a contradiction by supposing that there exists a tautological family $(F, \beta)$ on $\mathbb{C}$ (respectively on $\mathbb{A}_{\mathbb{C}, x}^{1}$ ). Then for each point $x \in \mathbb{C}$ (for each closed point $x \in \mathbb{A}_{\mathbb{C}, x}^{1}$ ) we have $J_{F}(x)=\tilde{J}\left(\left[F_{x}\right]\right)=\tilde{J}\left(\beta^{-1}(x)\right)$. Without loss of generality we may assume $\beta$ to be the underlying settheoretic map of $\tilde{J}: U / \operatorname{PSL}(2, \mathbb{Z}) \stackrel{\cong}{\longrightarrow} \mathbb{C}$ so that $J_{F}$ becomes simply the identity morphism on $\mathbb{C}$ (respectively on $\mathbb{A}_{\mathbb{C}, x}^{1}$ ). Now by the results of (4.7.2) we have for each $x \in \mathbb{C}$ (respectively for each closed point $x \in \mathbb{A}_{\mathbb{C}, x}^{1}$ ) a neighborhood $V$ and a holomorphic function (respectively a regular function) $\lambda$ on $V$ such that

$$
J_{F}(y)=\frac{4}{27} \frac{\left(1-\lambda(y)+(\lambda(y))^{2}\right)^{3}}{(\lambda(y))^{2}(1-\lambda(y))^{2}}=y
$$

for each $y \in V$ (respectively for each closed point $y \in V$ ). We consider two cases below.

Case 1: Local Obstruction at $x=0$. Consider the point $x=0$. Notice that this point corresponds to the elliptic curve with $j$-invariant 0 which admits extra automorphisms (see (4.8.1.2.1)). Since $J_{F}(0)=0$, the function $\lambda^{2}-\lambda+1$ vanishes at 0 and hence $J_{F}$ vanishes triply at 0 . But this cannot happen since $J_{F}$ is the identity morphism.

Case 2: Local Obstruction at $x=1$. Consider next the point $x=1$. Note again that this point corresponds to the elliptic curve with $j$-invariant 1 
which admits extra automorphisms (see (4.8.1.2.1)). Now we must have $\lambda(1)=-1$; for if $y \in V$ such that $\lambda(y)=-1$, then we have also $y=$ $J_{F}(y)=1$ by the formula which relates $\lambda$ locally to $J_{F}$ as above. Clearly the function $\left(J_{F}-1\right)$ vanishes at $x=1$. Further we have

$$
\left(J_{F}-1\right)^{\prime}=J_{F}^{\prime}=\frac{d J_{F}}{d x}=\frac{d J_{F}}{d \lambda} \cdot \frac{d \lambda}{d x} \quad \text { and }\left.\quad \frac{d J_{F}}{d \lambda}\right|_{(\lambda=-1)}=0
$$

so that $\left(J_{F}-1\right)^{\prime}$ also vanishes at $x=1$. Thus, $J_{F}$ assumes the value 1 with multiplicity greater than 1 which is again impossible since $J_{F}$ is the identity morphism.

From the above discussion, the following results are easily deduced.

4.8.1.3.1 Proposition. Let $T \in \mathcal{H}$ (respectively $T \in \mathcal{A})$ and $F$ be a family parametrized by $T$ i.e., $F \in \mathbb{M}_{1}^{H O L}(T)$ (respectively $F \in \mathbb{M}_{1}^{A L G}(T)$ ). Then $J_{F}$ is ramified at each point $t \in T$ (respectively at each closed point $t \in T$ ) at which $J_{F}$ assumes either of the values 0 or 1 .

4.8.1.3.2 Note. This proposition implies that the natural transformations $J^{H O L}$ and $J^{A L G}$ cannot be surjective. Hence the pairs $\left(\mathbb{C}, J^{H O L}\right)$ and $\left(\mathbb{A}_{\mathbb{C}, x}^{1}, J^{A L G}\right)$ cannot be fine moduli spaces.

4.8.1.3.3 Theorem (Local Obstruction to Existence of Tautological Families). It is impossible to find any neighborhood $V$ of any given point of $\mathbb{C}$ (respectively of any given closed point of $\mathbb{A}_{\mathbb{C}, x}^{1}$ ) and a family $F$ parametrized by $V$ whose fibers are mutually non-isomorphic (as elliptic curves) and such that an elliptic curve with extra automorphism occurs as one of the fibers of $F$.

Compare the above result with the remarks at the end of subsection 2.6.2.

4.8.1.3.4 Corollary. The pairs $\left(\mathbb{C}, J^{H O L}\right)$ and $\left(\mathbb{A}_{\mathbb{C}, x}^{1}, J^{A L G}\right)$ cannot be fine moduli spaces.

4.8.1.3.5 Remark. It is natural to hope that if we exclude the elliptic curves admitting extra automorphisms from the present classification, i.e., consider the moduli problem of parametrizing the set of elliptic curves with $j$-invariant different from 0 and 1 , then there may exist a tautological family. This turns out to be true and it can be shown that there exists a tautological family in this case on $\mathbb{C}-\{0,1\}$ for the holomorphic version of this moduli problem and on $C=\operatorname{Spec}\left(\mathbb{C}\left[x,(x(1-x))^{-1}\right]\right)$ for the algebraic version. See for example Mumford's lecture [24].

We next turn to describe "global obstructions" to the existence of a fine moduli space for the problem of moduli of elliptic curves. 


\subsubsection{Global Obstructions to Existence of a Fine Moduli Space}

We have seen in the previous subsection that the coarse moduli spaces

$$
\left(\mathbb{C}, J^{H O L}\right) \text { and }\left(\mathbb{A}_{\mathbb{C}, x}^{1}, J^{A L G}\right)
$$

cannot be fine moduli spaces. We now show that there can never exist fine moduli spaces for these moduli problems. We will prove this by showing the existence of certain families — "global obstructions" — which will contradict the existence of a universal family on the classifying space of any given coarse moduli space.

The discussion below depends on the fact that there exists a nontrivial automorphism on any elliptic curve (see (4.8.1.2.1)). The presence of such an automorphism allows us to construct a family $F$, parametrized by a suitable base $T$, which is locally trivial but not globally trivial. Now if $(M, \Phi)$ were a coarse moduli space, then for any chosen point $t \in|T|$, we have the equality of morphisms (since $F$ is locally trivial)

$$
\begin{aligned}
(\Phi(T))([F]) & =(\Phi(T))\left(\left[T \times F_{t}\right]\right) \\
& =\text { constant morphism } \phi: T \longrightarrow M, t^{\prime} \mapsto \Phi(P)\left(\left[F_{t}\right]\right),
\end{aligned}
$$

where $T \times F_{t} \stackrel{p_{1}}{\longrightarrow} T$ denotes the trivial family parametrized by $T$ with fiber type $F_{t}$. However, we also have that $F$ is not equivalent (under $\sim_{T}$ ) to $T \times F_{t}$ since $F$ is not globally trivial. Thus we see that $\Phi(T)$ itself is not injective. Hence $(M, \Phi)$ cannot be a fine moduli space. Notice also that requiring that $(M, \Phi)$ be a fine moduli space is equivalent to requiring that there exist a universal family $U$ on $M$ (see (4.5.3.3.2)). But if such a $U$ existed, then we would have (by the very definition of a universal family) that $F \sim_{T} \phi^{*}(U) \sim_{T} T \times F_{t}$ which is a contradiction.

\subsubsection{Remarks.}

1. Such an $F$ as above may thus be called a global obstruction to the existence of a fine moduli space.

2. More generally, using an argument similar to the above, we may show the existence of global obstructions whenever some of the objects to be parametrized admit nontrivial automorphisms.

3. The above argument also shows that we would be unable to obtain a fine moduli space (i.e., a universal family) even if we exclude the elliptic curves with extra automorphisms, though we showed earlier that in this case there do exist tautological families. 
The only assertion we need to prove in order to substantiate the discussion above is that there exists a locally trivial family of elliptic curves which is not globally trivial.

To this end, let $T$ be a smooth connected object of $\mathcal{H}$ (respectively a smooth irreducible object of $\mathcal{A}$ ) which admits a fixed-point-free involutory automorphism $\tau: T \stackrel{\cong}{\longrightarrow}$. Thus $G_{\tau}:=\left\{1_{T}, \tau\right\}$ is a finite subgroup of automorphisms of $T$ and acts in a "good" manner (for example, properly discontinuously in the context of the category $\mathcal{H}$ ) and without fixed points on $T$, so that the quotient $T / G_{\tau}$ is also an object of $\mathcal{H}$ (respectively an object of $\mathcal{A})$.

The canonical quotient map $T \longrightarrow T / G_{\tau}$ is a morphism and may be considered as a principal $G_{\tau}$-bundle on $T / G_{\tau}$ (for example in the context of the category $\mathcal{H}$, this canonical quotient map is a covering space map with the fundamental group of $T / G_{\tau}$ isomorphic to $G_{\tau}$ acting as the deck transformation group of this covering — see (1.5.2.1.2)).

Next pick any elliptic curve $E$ and let $-1_{E}$ be the nontrivial involutory automorphism of $E$ generating the subgroup $H:=\left\{1_{E},-1_{E}\right\}$ of $\operatorname{Aut}(E)$. Define the following isomorphism of groups

$$
\psi: G_{\tau} \longrightarrow H, \tau \mapsto-1_{E}
$$

Consider the action of $G_{\tau}$ on the product $T \times H$ defined as follows:

$$
G_{\tau} \times(T \times H) \longrightarrow T \times H,(g,(t, h)) \mapsto g \cdot(t, h):=\left(\tau(t), \psi(g)^{-1} \cdot h\right) .
$$

Then one can prove (say for example using the Theory of Descent of principal bundles) that the quotient $F:=(T \times H) / G_{\tau}$ gives rise to a locally trivial bundle on $T / G_{\tau}$ whose fibers are all elliptic curves isomorphic to $E$ and that this bundle is not globally trivial. Thus $p: F \longrightarrow T / G_{\tau}$ (where the morphism $p$ is the one naturally induced from $T \times E \stackrel{p_{1}}{\longrightarrow} T$ considered as trivial bundle over $T$ with fiber type $E$ ) is a locally trivial family of elliptic curves which is not globally trivial. That $F$ is locally trivial in the context of $\mathcal{H}$ also follows from a theorem of Grauert and Fischer [12] once we verify that $F$ is a family parametrized by $T / G_{\tau} \in \mathcal{H}$. 


\section{Appendix: Analytic Spaces, Schemes and Cohomology}

The aim of the following presentation is to quickly recall some fundamental definitions and results concerning complex analytic spaces, schemes, cohomology and algebraizability that are relevant to this book. For more details, refer to the textbook of R.Hartshorne on Algebraic Geometry and the textbook of Grauert and Remmert on Coherent Analytic Sheaves listed under the References at the end.

\section{A.1 Sheaves}

Let $X$ be a topological space. Define the category $\mathcal{T}_{X}$ whose objects are open subsets of $X$ and whose only morphisms are the inclusions of open sets. Let $\mathcal{C}$ be any category whose objects are sets with some additional mathematical structure.

By a presheaf $\mathcal{F}$ of objects of $\mathcal{C}$ on $X$ we mean a contravariant functor $\mathcal{F}: \mathcal{T}_{X} \longrightarrow \mathcal{C}$. For an open subset $U$ of $X$, the elements of $\mathcal{F}(U)$ are called sections of $\mathcal{F}$ over $U$. If $V \hookrightarrow U$ is an inclusion of open subsets of $X$, then the map induced by the functor $\mathcal{F}$ from $\mathcal{F}(U)$ to $\mathcal{F}(V)$ is called the restriction map and the image of a section $s$ under this map is often denoted by $\left.s\right|_{V}$.

Next, given a presheaf $\mathcal{F}$ as above, we call it a sheaf if it satisfies the following two conditions:

1. if $U$ is any open subset of $X$ and if $\left\{V_{i} ; i \in I\right\}$ is an open covering of $U$, then two sections over $U$, which when restricted to each $V_{i}$ become equal, must already be equal in $U$; in other words, two sections are the same if they are locally the same; 
2. with $U$ and $V_{i}$ as above, if we are prescribed sections, one over each $V_{i}$, which when restricted to the intersections of the $V_{i}$ 's agree, then there must be a section over $U$ which when restricted to each $V_{i}$ gives the prescribed sections (note that by (1) above, this section over $U$ must be unique); in other words, a section needs to be defined only locally in a compatible way - this automatically defines the section globally.

We therefore see that sheaves are those presheaves which depend only on local data.

Henceforth we shall assume that in the category $\mathcal{C}$, every direct system has a direct limit. Then for a presheaf $\mathcal{F}$ of objects of $\mathcal{C}$ on $X$ and for a point $x \in X$, we define the stalk of $\mathcal{F}$ at $x$ to be the direct limit of the direct system consisting of the objects $\mathcal{F}(U)$ (where $U$ is an open subset of $X$ containing $x$ ) alongwith the corresponding restriction maps. The stalk of $\mathcal{F}$ at $x$ thus obtained is also an object of $\mathcal{C}$ and is denoted $\mathcal{F}_{x}$.

By the universal property of direct limits in the category $\mathcal{C}$, this stalk is uniquely determined with canonical maps (which we call the limiting restriction maps) from $\mathcal{F}(U)$ to $\mathcal{F}_{x}$ for each open subset $U$ of $X$ which is a neighborhood of $x$. For such $U$, the image of a section $s$ over $U$ in the stalk by the limiting restriction map is called the germ of $s$ at $x$ and is denoted $s_{x}$.

A morphism of presheaves or sheaves is simply defined to be a morphism of functors. Clearly, such a morphism induces morphisms of the corresponding stalks (of the presheaves at every point of $X$ ) because of universal properties of direct limits. We note the following standard result.

A morphism of sheaves is an isomorphism if and only if each of the induced morphisms of stalks is an isomorphism.

Since there exist presheaves that are not sheaves, we must note that this result cannot hold for presheaves. In order to deal with presheaves that are not sheaves, we have the following result.

Given a presheaf $\mathcal{F}$ of objects of $\mathcal{C}$ on $X$, there exists a sheaf $\tilde{\mathcal{F}}$ (of objects of $\mathcal{C}$ ) on $X$ and a morphism of presheaves $\theta: \mathcal{F} \longrightarrow$ $\tilde{\mathcal{F}}$ satisfying the following universal property. If $\mathcal{G}$ is a sheaf of objects of $\mathcal{C}$ on $X$ and $\phi: \mathcal{F} \longrightarrow \mathcal{G}$ is a morphism of presheaves, then there exists a unique morphism $\psi: \tilde{\mathcal{F}} \longrightarrow \mathcal{G}$ such that $\phi=\psi \circ \theta$. The pair $(\tilde{\mathcal{F}}, \theta)$ is thus defined uniquely up to a unique isomorphism and $\tilde{\mathcal{F}}$ is called the sheaf associated to the presheaf $\mathcal{F}$ or the sheafification of the presheaf $\mathcal{F}$. Further, at each point of $X$, the stalks of $\mathcal{F}$ and $\tilde{\mathcal{F}}$ are the same. Also, $\mathcal{F}$ itself is a sheaf iff $\theta$ is an isomorphism of presheaves. 
The advantage of the above result is explained in the following. Suppose we have an operation defined on objects of $\mathcal{C}$ - say $\mathcal{C}$ is the category whose objects are abelian groups with group homomorphisms for morphisms and consider the operation of taking the quotient of an abelian group by a subgroup. Then we would like to define a corresponding operation on sheaves of objects of $\mathcal{C}$ on $X$. It usually happens that the natural definition gives only a presheaf and not a sheaf: suppose $\mathcal{F}$ and $\mathcal{G}$ are sheaves of abelian groups on $X$ such that the former is a subsheaf of the latter i.e., $\mathcal{F}(U)$ is a subgroup of $\mathcal{G}(U)$ for each open subset $U$ of $X$; then we would naturally be led to define the quotient $\mathcal{G} / \mathcal{F}$ by the association $U \mapsto \mathcal{G}(U) / \mathcal{F}(U)$ which unfortunately only gives a presheaf! The right way out of this problem is to take the sheaf associated to the presheaf that we obtained in the first place. Then it also turns out that this technique suffices for the most general applications of the Theory of Sheaves.

Now let $f: X \longrightarrow Y$ be a continuous map of topological spaces. Let $\mathcal{F}$ be a sheaf of objects of $\mathcal{C}$ on $X$. Define the association $V \mapsto \mathcal{F}\left(f^{-1}(V)\right)$ for each open subset $V$ of $Y$. This is a sheaf of objects of $\mathcal{C}$ on $Y$ denoted $f_{*} \mathcal{F}$ and is called the direct image sheaf or the pushforward of the sheaf $\mathcal{F}$ by $f$.

Next, let $\mathcal{G}$ be a sheaf of objects of $\mathcal{C}$ on $Y$. Pick an open subset $U$ of $X$. Let $V$ be an open subset of $Y$ such that $f(U) \subseteq V$. Then for such $V$, the objects $\mathcal{G}(V)$ alongwith the restriction maps form a direct system in $\mathcal{C}$ and hence have a direct limit which we denote as $\left(f^{-1} \mathcal{G}\right)_{P R E}(U)$. Then the association $U \mapsto\left(f^{-1} \mathcal{G}\right)_{P R E}(U)$ defines a presheaf. We denote by $f^{-1} \mathcal{G}$ the sheaf associated to this presheaf and call it the inverse image sheaf of $\mathcal{G}$ under $f$ or the pullback of $\mathcal{G}$ by $f$.

Let Sheaves $(X, \mathcal{C})$ denote the category of sheaves of objects of $\mathcal{C}$ on $X$. Then we see that the association

$$
f_{*}: \operatorname{Sheaves}(X, \mathcal{C}) \longrightarrow \operatorname{Sheaves}(Y, \mathcal{C})
$$

given by $\mathcal{F} \mapsto f_{*} \mathcal{F}$ and the association

$$
f^{-1}: \operatorname{Sheaves}(Y, \mathcal{C}) \longrightarrow \operatorname{Sheaves}(X, \mathcal{C})
$$

given by $\mathcal{G} \mapsto f^{-1} \mathcal{G}$ are functors.

A.1.1 Note. If $X$ is a topological space and $Y \subseteq X$ is a subset with inclusion map $i: Y \hookrightarrow X$ and $\mathcal{F}$ is a sheaf on $X$, then we call the inverse image sheaf $i^{-1} \mathcal{F}$ as the restriction of $\mathcal{F}$ to the topological subspace $Y$. This restricted sheaf is often denoted by $\left.\mathcal{F}\right|_{Y}$. The stalk of the restricted sheaf at a point of $Y$ is the same as that of the original sheaf at that point. 


\section{A.2 Locally Ringed Spaces}

A ringed space is a pair $\left(X, \mathcal{O}_{X}\right)$ consisting of a topological space $X$ and a sheaf of rings $\mathcal{O}_{X}$ on $X$. In the notation of the previous section, $\mathcal{O}_{X}$ is an element of $\operatorname{Sheaves}(X, \mathcal{R})$, where $\mathcal{R}$ is the category of commutative rings and ring homomorphisms. A morphism of ringed spaces from $\left(X, \mathcal{O}_{X}\right)$ to $\left(Y, \mathcal{O}_{Y}\right)$ is a pair $\left(f, f^{\#}\right)$ consisting of a continuous map $f: X \longrightarrow Y$ and a map $f^{\#}: \mathcal{O}_{Y} \longrightarrow f_{*} \mathcal{O}_{X}$ of sheaves of rings on $Y$. Here $f_{*} \mathcal{O}_{X}$ is the pushforward of $\mathcal{O}_{X}$ by $f$ (see $(\mathrm{A} .1)$ ). The ringed space $\left(X, \mathcal{O}_{X}\right)$ is called a locally ringed space if for each point $x \in X$, the stalk $\mathcal{O}_{X, x}$ (see (A.1)) is a local ring.

A morphism of locally ringed spaces is a morphism $\left(f, f^{\#}\right)$ of ringed spaces as above, such that for each point $x \in X$, the canonically induced map of local rings $f_{x}^{\#}: \mathcal{O}_{Y, f(x)} \longrightarrow \mathcal{O}_{X, x}$ is a local homomorphism of local rings in the sense that the inverse image of the maximal ideal of the target local ring (under this homomorphism of rings) is precisely the maximal ideal of the source local ring. An isomorphism of locally ringed spaces is a morphism with a two-sided inverse. Thus a morphism of locally ringed spaces $\left(f, f^{\#}\right)$ is an isomorphism if and only if $f$ is a homeomorphism of the underlying topological spaces and $f^{\#}$ is an isomorphism of sheaves.

Let $\left(f, f^{\#}\right):\left(X, \mathcal{O}_{X}\right) \longrightarrow\left(Y, \mathcal{O}_{Y}\right)$ be a morphism of locally ringed spaces. For any $x \in X$, we have the local homomorphism of local rings $f^{\#}: \mathcal{O}_{Y, y} \longrightarrow \mathcal{O}_{X, x}$ which makes $\mathcal{O}_{X, x}$ a module over $\mathcal{O}_{Y, y}$ where $y=f(x)$. We say that $f$ is flat if for each $x \in X, \mathcal{O}_{X, x}$ is flat as an $\mathcal{O}_{Y, y}$-module.

\section{A.3 Pullbacks and Pushforwards of Sheaves of Modules}

Let $\left(X, \mathcal{O}_{X}\right)$ be a ringed space as defined in (A.2). A sheaf $\mathcal{F}$ of abelian groups on $X$ is said to be a sheaf of $\mathcal{O}_{X}$-modules if for every open set $U \subset X$, the abelian group $\mathcal{F}(U)$ is a module over the ring $\mathcal{O}_{X}(U)$; further, for an inclusion of open sets $V \hookrightarrow U$, since the module $\mathcal{F}(V)$ over the ring $\mathcal{O}_{X}(V)$ gets, via the restriction map of rings $\mathcal{O}_{X}(U) \rightarrow \mathcal{O}_{X}(V)$, the structure of a module over $\mathcal{O}_{X}(U)$, it is required that the restriction $\mathcal{F}(U) \rightarrow \mathcal{F}(V)$ be a homomorphism of $\mathcal{O}_{X}(U)$-modules. A sheaf of $\mathcal{O}_{X}$-modules is often simply referred to as an $\mathcal{O}_{X}$-module. A morphism of $\mathcal{O}_{X}$-modules $f: \mathcal{F} \longrightarrow \mathcal{G}$ is a morphism of sheaves with the additional condition that for any open subset $U$ of $X$, the homomorphism of abelian groups $f(U): \mathcal{F}(U) \longrightarrow \mathcal{G}(U)$ be a morphism of $\mathcal{O}_{X}(U)$-modules. Let 


$$
\left(f, f^{\#}\right):\left(X, \mathcal{O}_{X}\right) \longrightarrow\left(Y, \mathcal{O}_{Y}\right)
$$

be a morphism of ringed spaces (as defined in the previous section) and let $\mathcal{F}$ be an $\mathcal{O}_{X}$-module. Then the pushforward sheaf $f_{*} \mathcal{F}$ (cf. (A.1)) is an $f_{*} \mathcal{O}_{X}$-module, hence via the morphism of sheaves of rings on $Y$ given by $f^{\#}$, it becomes an $\mathcal{O}_{Y}$-module. This $\mathcal{O}_{Y}$-module is called the direct image $\mathcal{O}_{Y}$-module of $\mathcal{F}$ by $f$ or the pushforward of $\mathcal{F}$ by $f$.

Next let $\mathcal{G}$ be a sheaf of $\mathcal{O}_{Y}$-modules. Then the inverse image sheaf $f^{-1} \mathcal{G}$ (cf. (A.1)) is a sheaf of $f^{-1} \mathcal{O}_{Y}$-modules. If we denote by $\operatorname{HOM}_{S h(X)}(\mathcal{L}, \mathcal{M})$ the set of morphisms of sheaves (of objects of a fixed category) on $X$, then we have for any sheaf $\mathcal{F}$ on $X$ and any sheaf $\mathcal{G}$ on $Y$ a canonical bijection

$$
\operatorname{HOM}_{S h(X)}\left(f^{-1} \mathcal{G}, \mathcal{F}\right) \cong \operatorname{HOM}_{S h(Y)}\left(\mathcal{G}, f_{*} \mathcal{F}\right)
$$

Thus we have in particular

$$
\operatorname{HOM}_{S h(X)}\left(f^{-1} \mathcal{O}_{Y}, \mathcal{O}_{X}\right) \cong \operatorname{HOM}_{S h(Y)}\left(\mathcal{O}_{Y}, f_{*} \mathcal{O}_{X}\right)
$$

Therefore $f^{\#}$ defines (by the above bijection) a unique morphism of sheaves of rings from $f^{-1} \mathcal{O}_{Y} \longrightarrow \mathcal{O}_{X}$ which makes $\mathcal{O}_{X}$ an $f^{-1} \mathcal{O}_{Y}$-module. Since the inverse image sheaf $f^{-1} \mathcal{G}$ is an $f^{-1} \mathcal{O}_{Y}$-module, we can define the tensor product

$$
\left(f^{-1} \mathcal{G}\right) \otimes_{\left(f^{-1} \mathcal{O}_{Y}\right)} \mathcal{O}_{X}
$$

bearing in mind the remarks in (A.1) on dealing with naturally defined presheaves that are not sheaves. This tensor product is naturally an $\mathcal{O}_{X^{-}}$ module, denoted $f^{*} \mathcal{G}$ and is called the inverse image $\mathcal{O}_{X}$-module of $\mathcal{G}$ by $f$ or the pullback of $\mathcal{G}$ by $f$.

It then turns out that for any $\mathcal{O}_{X}$-module $\mathcal{F}$ and any $\mathcal{O}_{Y}$-module $\mathcal{G}$, there is a natural isomorphism of abelian groups

$$
\operatorname{HOM}_{S h\left(\mathcal{O}_{X}\right)}\left(f^{*} \mathcal{G}, \mathcal{F}\right) \cong \operatorname{HOM}_{S h\left(\mathcal{O}_{Y}\right)}\left(\mathcal{G}, f_{*} \mathcal{F}\right)
$$

where $\operatorname{HOM}_{S h\left(\mathcal{O}_{X}\right)}(\mathcal{L}, \mathcal{M})$, for any $\mathcal{O}_{X}$-modules $\mathcal{L}$ and $\mathcal{M}$, denotes the additive abelian group (in fact module over the $\operatorname{ring} \mathcal{O}_{X}(X)$ ) of morphisms of $\mathcal{O}_{X}$-modules.

\section{A.4 Examples of Locally Ringed Spaces}

The following examples of locally ringed spaces are fundamental in the sense that they form the local models based on which structures like manifolds, analytic spaces and schemes are defined. 


\section{A.4.1 The Local Model for Differentiable Manifolds}

Let $X$ be a domain in $\mathbb{R}^{n}$. Let $\mathcal{D}_{X}$ denote the sheaf of germs of $C^{\infty}$ functions on $X$. This means that $\mathcal{D}_{X}$ associates to each open subset $V$ of $X$, the ring of $C^{\infty}$ functions defined on $V$. Then $\left(X, \mathcal{D}_{X}\right)$ is a locally ringed space.

\section{A.4.2 The Local Model for Complex Manifolds}

Let $X$ be a domain in $\mathbb{C}^{n}$. Let $\mathcal{O}_{X}$ denote the sheaf of germs of holomorphic functions on $X$. Then $\left(X, \mathcal{O}_{X}\right)$ is a locally ringed space.

\section{A.4.3 The Local Model for Schemes}

Let $A$ be a commutative ring with (multiplicative) identity element 1 . Let $\operatorname{Spec}(A)$ denote the set of all prime ideals of $A$. For any ideal $I$ of $A$, define the subset $V(I)$ of $\mathbf{S p e c}(A)$ to consist of those prime ideals that contain $I$. Define a topology on $\operatorname{Spec}(A)$ by taking subsets of the form $V(I)$ to be the closed sets - this gives the Zariski topology on $X:=\operatorname{Spec}(A)$.

For any $f \in A$, let $D(f)=\operatorname{Spec}(A) \backslash V(\langle f\rangle)$ where $\langle f\rangle$ is the ideal generated by $f \in A$. Then $D(f)$ is an open set and sets of this type form a base for the Zariski topology on $X$.

Next define the association $D(f) \mapsto A_{f}$ where $A_{f}$ denotes the localization of the ring $A$ with respect to the multiplicative system $\left\{1, f, f^{2}, \ldots\right\}$. Since the subsets of the form $D(f)$ form a base for the Zariski topology on $X$, this association is enough to define a unique sheaf of local rings on $X$ which we denote by $\mathcal{O}_{X}$ and such that the ring of sections of this sheaf over the open set $D(f)$ is isomorphic to $A_{f}$, for each $f \in A$. This sheaf of local rings is called the structure sheaf of regular functions on $X$.

Then $\left(X, \mathcal{O}_{X}\right)$ is a locally ringed space and is called the affine scheme defined by the ring $A$.

Consider the polynomial ring $\mathbb{C}\left[x_{1}, \ldots, x_{n}\right]$ in $n$ indeterminates over $\mathbb{C}$. Then the underlying set of closed points of the affine scheme $\mathbb{A}_{\mathbb{C}}^{n}:=$ Spec $\mathbb{C}\left[x_{1}, \ldots, x_{n}\right]$ can be canonically identified with the points of the $n$ dimensional complex space $\mathbb{C}^{n}$. Thus $\mathbb{A}_{\mathbb{C}}^{n}$ is called the scheme (structure) associated to $\mathbb{C}^{n}$.

\section{A.4.4 The Local Model for Complex Analytic Spaces}

Let $U \subset \mathbb{C}^{n}$ be a domain and let $f_{1}, \ldots, f_{q}$ be holomorphic functions on $U$. Let $X \subset U$ be the analytic subset consisting of common zeros of these holomorphic functions. Let $\mathcal{O}_{U}$ denote the sheaf of germs of holomorphic functions on $U$. Set $\mathcal{I}=\mathcal{O}_{U} f_{1}+\cdots+\mathcal{O}_{U} f_{q}$ which is the ideal sheaf in $\mathcal{O}_{U}$ generated by $f_{1}, \ldots, f_{q}$. This is referred to as a coherent analytic ideal 
sheaf. Consider the quotient sheaf of rings $\mathcal{O}_{U} / \mathcal{I}$ and let $\mathcal{O}_{X}$ denote the restriction of this to $X$. Then $\left(X, \mathcal{O}_{X}\right)$ is a locally ringed space.

\section{A.5 Manifolds, Analytic Spaces and Schemes as Locally Ringed Spaces}

We use the local models defined in (A.4) above to define the following.

\section{A.5.1 Definition of Differentiable Manifold of Class $C^{\infty}$}

A locally ringed space $\left(Y, \mathcal{O}_{Y}\right)$ which is locally isomorphic as locally ringed space to a locally ringed space of the form $\left(X, \mathcal{D}_{X}\right)$ as in (A.4.1) above is called a differentiable manifold of class $C^{\infty}$.

\section{A.5.2 Definition of Complex Manifold}

A locally ringed space $\left(Y, \mathcal{O}_{Y}\right)$ which is locally isomorphic as locally ringed space to a locally ringed space of the form $\left(X, \mathcal{O}_{X}\right)$ as in (A.4.2) above is called a complex manifold.

\section{A.5.3 Definition of Scheme}

A locally ringed space $\left(Y, \mathcal{O}_{Y}\right)$ which is locally isomorphic as locally ringed space to a locally ringed space of the form $\left(X, \mathcal{O}_{X}\right)$ as in $(\mathrm{A} .4 .3)$ above is called a scheme.

\section{A.5.4 Definition of Complex Analytic Space}

A locally ringed space $\left(Y, \mathcal{O}_{Y}\right)$ which is locally isomorphic as locally ringed space to a locally ringed space of the form $\left(X, \mathcal{O}_{X}\right)$ as in $(\mathrm{A} .4 .4)$ above is called a complex analytic space.

A.5.5 Remark. For each of the mathematical structures defined above, we have not indicated the definition of a morphism between two spaces $\left(Y, \mathcal{O}_{Y}\right)$ and $\left(Y^{\prime}, \mathcal{O}_{Y^{\prime}}\right)$ having that structure. However, since these spaces are both locally ringed spaces, we do have the notion of a morphism of locally ringed spaces as explained in (A.2). It turns out that this notion is all we need to define a morphism, irrespective of what type of ringed space we are dealing with. In other words, for e.g., if both these spaces are complex manifolds, then the underlying topological map, of a morphism of locally ringed spaces between these two spaces, itself has the property that 
it is a holomorphic map. This is one of the great advantages of regarding manifolds, schemes and analytic spaces as locally ringed spaces.

\section{A.6 Some Definitions from Scheme Theory}

\section{A.6.1 Affine Schemes and Commutative Rings}

Since the inverse image of a prime ideal under a ring homomorphism is again a prime ideal, given a map of commutative rings $\phi: A \longrightarrow B$ which respects the identity elements for multiplication, there is an obvious map of topological spaces

$$
f_{\phi}: \operatorname{Spec}(B) \longrightarrow \operatorname{Spec}(A)
$$

and a morphism of sheaves of rings

$$
f_{\phi}^{\#}: \mathcal{O}_{\mathbf{S p e c}(A)} \longrightarrow f_{*} \mathcal{O}_{\mathbf{S p e c}(B)}
$$

such that $\left(f_{\phi}, f_{\phi}^{\#}\right)$ is a morphism of locally ringed spaces from $\left(\operatorname{Spec}(B), \mathcal{O}_{\mathbf{S p e c}(B)}\right)$ to $\left(\operatorname{Spec}(A), \mathcal{O}_{\mathbf{S p e c}(A)}\right)$. By $($ A.5.5) above, this is a morphism of affine schemes (cf. (A.4.3)).

Let $\mathcal{R}$ denote the category whose objects are commutative rings with identity element for multiplication and whose morphisms are ring homomorphisms that preserve multiplicative identity elements. Let $\mathcal{A}$ be the category of affine schemes. Then the contravariant functor from $\mathcal{R}$ to $\mathcal{A}$ given by $A \mapsto\left(\operatorname{Spec}(A), \mathcal{O}_{\mathbf{S p e c}(A)}\right)$ and $(\phi: A \longrightarrow B) \mapsto\left(f_{\phi}, f_{\phi}^{\#}\right)$ as defined above is an equivalence of categories. Thus, morphisms of schemes are locally dictated by homomorphisms of commutative rings.

\section{A.6.2 Integral Schemes}

A scheme $\left(X, \mathcal{O}_{X}\right)$ is said to be reduced if for each open set $U \subseteq X$, the $\operatorname{ring} \mathcal{O}_{X}(U)$ is reduced i.e., has no nilpotent elements or equivalently if for each point $x \in X$, the local ring $\mathcal{O}_{X, x}$ is reduced.

A scheme $\left(X, \mathcal{O}_{X}\right)$ is said to be connected (respectively irreducible) if its underlying topological space is connected (respectively irreducible). A topological space is said to be irreducible if it is not the union of two distinct nontrivial proper closed subsets.

A scheme that is both reduced and irreducible is said to be integral - in this case the ring of regular functions $\mathcal{O}_{X}(U)$ on any open subset $U \subset X$ is an integral domain. 


\section{A.6.3 Schemes of Finite Type over $\mathbb{C}$, Open and Closed Subschemes}

A scheme $\left(X, \mathcal{O}_{X}\right)$ is said to be a scheme of finite type over $\mathbb{C}$ if it can be covered by finitely many affine open subschemes each of which is defined by a finitely generated algebra over $\mathbb{C}$. The morphism of schemes from $X$ to $\operatorname{Spec}(\mathbb{C})$ is called the structure morphism of $X$. Here by an open subscheme we mean the canonical structure of scheme $\left(U, \mathcal{O}_{U}:=\left.\mathcal{O}_{X}\right|_{U}\right)$ induced on an open subset $U$ of the topological space underlying the scheme.

A morphism of schemes $\left(f, f^{\#}\right):\left(X, \mathcal{O}_{X}\right) \longrightarrow\left(Y, \mathcal{O}_{Y}\right)$ is said to be an open immersion (respectively closed immersion) if $f(X)$ is an open subset (respectively closed subset) of the topological space $Y$ and $\left(f, f^{\#}\right)$ : $\left(X, \mathcal{O}_{X}\right) \longrightarrow\left(f(X), \mathcal{O}_{f(X)}\right)$ is an isomorphism (respectively if $f$ is a homeomorphism onto $f(X)$ and $f^{\#}$ is surjective).

A closed subscheme is an equivalence class of closed immersions, where two closed immersions are said to be equivalent if their source schemes are isomorphic by a morphism that is compatible with the morphisms that define the closed immersions.

\section{A.6.4 Local Structure of Closed Subschemes}

If $A$ is a commutative ring with identity element for multiplication, $I \subset A$ an ideal, $V(I)$ the closed subset of $\mathbf{S p e c}(A)$ in the Zariski topology that we defined earlier (see (A.4.3)), then the locally ringed space

$$
\left(\operatorname{Spec}(A / I), \mathcal{O}_{\operatorname{Spec}(A / I)}\right)
$$

gives the structure of a closed subscheme of $\left(\mathbf{S p e c}(A), \mathcal{O}_{\mathbf{S p e c}(A)}\right)$ on $V(I)$. Every representative of a closed subscheme of a given scheme is isomorphic to such a scheme $V(I)$, when restricted to any affine open subscheme $\operatorname{Spec}(A)$ of the given scheme.

$$
\begin{gathered}
\text { Hereafter we shorten the notation } \\
\left(f, f^{\#}\right):\left(X, \mathcal{O}_{X}\right) \longrightarrow\left(Y, \mathcal{O}_{Y}\right) \text { to } f: X \longrightarrow Y .
\end{gathered}
$$

\section{A.6.5 Fiber Products}

Let $f: X \longrightarrow Y$ and $g: Z \longrightarrow Y$ be morphisms of schemes. Then there exists a scheme (unique up to a unique isomorphism) denoted by $X \times_{Y} Z$ which is a universally attracting object $P$ in the category of schemes $F$ together with morphisms $f_{F}^{\prime}$ and $g_{F}^{\prime}$ that make the following diagram commute: 


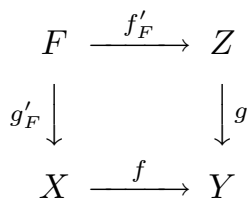

This scheme $X \times_{Y} Z$ is called the fiber product of $X$ with $Z$ over $Y$ via $f$ and $g$; further, $g_{P}^{\prime}$ (respectively $f_{P}^{\prime}$ ) is said to be obtained from $g$ (respectively from $f$ ) by extending the base $Y$ to $X$ via $f$ (respectively by extending the base $Y$ to $Z$ via $g$ ).

\section{A.6.6 Separated Schemes}

Let $X$ be a scheme of finite type over $\mathbb{C}$ and $X \times X$ denote the fiber product of $X$ with itself over $\operatorname{Spec}(\mathbb{C})$ via the canonical structure morphism from $X$ into the one-point-scheme $\operatorname{Spec}(\mathbb{C})$. Let $\Delta: X \longrightarrow X \times X$ be the diagonal morphism. The scheme $X$ is said to be separated over $\mathbb{C}$ if $\Delta$ is a closed immersion (see (A.6.3)) or equivalently if the image of $\Delta$ is closed.

\section{A.6.7 Proper Morphisms and Projective Schemes}

A morphism of schemes $f: X \longrightarrow Y$ is said to be proper if the following hold:

1. the diagonal morphism from $X$ into the fiber product of $X$ with itself over $Y$ via the map $f$ is a closed immersion;

2. the inverse image of any open affine subscheme of $Y$ is covered by finitely many open affine subschemes of $X$ for each of which the defining commutative ring is a finitely generated algebra (by the ring homomorphism given by $f$ locally) over the commutative ring defining the open affine subscheme of $Y$ in consideration (cf. (A.4.3), (A.6.1) and (A.6.3));

3. $f$ as a map of underlying topological spaces is a closed map;

4. for any morphism of schemes $g: Z \longrightarrow Y$, the morphism $f^{\prime}: Z \times_{Y}$ $X \longrightarrow Z$ obtained by base extension (cf. (A.6.5) above) is also a closed map when considered just as a map of the underlying topological spaces.

Consider the polynomial ring $S:=\mathbb{C}\left[x_{0}, \ldots, x_{n}\right]$ in $(n+1)$ indeterminates over $\mathbb{C}$. This is an $\mathbb{Z}$-graded ring with the graded piece of elements of degree $m \in \mathbb{Z}$ being the $\mathbb{C}$-vector space of homogeneous polynomials of degree $m$ if $m$ is a positive integer, $\mathbb{C}$ if $m=0$ and the trivial vector space (0) if $m<0$.

Let $\operatorname{Proj}(S)$ be the set of all homogeneous prime ideals $I \subset S$ which do not contain the ideal $S_{+}:=\oplus_{d \geq 1} S_{d}$. Further we define a topology on 
$\operatorname{Proj}(S)$ by taking closed sets to be of the form $V(I):=\{J \in \operatorname{Proj}(S) ; I \subseteq$ $J\}$ for some homogeneous ideal $I \subset S$. Then for any homogeneous $f \in S_{+}$ the set $D_{+}(f):=\{J \in \operatorname{Proj}(S) ; f$ not in $J\}=\operatorname{Proj}(S) \backslash V(\langle f\rangle)$, where $\langle f\rangle$ is the homogeneous ideal generated by $f$ in $S$, is open and such sets form a base for the topology on $\operatorname{Proj}(S)$.

Next for such a homogeneous element $f$, let $S_{f}$ be the localisation of $S$ at (the multiplicative system consisting of nonnegative integral powers of) $f$ and let $S_{(f)}$ be the subring of $S_{f}$ consisting of degree zero elements $\left(S_{f}\right.$ canonically acquires the structure of a graded ring from $S$ ). There is a canonical bijection between elements of $\operatorname{Spec}\left(S_{(f)}\right)$ and the elements of $D_{+}(f)$ intersected with $S_{(f)}$. Hence, using this bijection, we can transport the structure of locally ringed space on the affine scheme $\operatorname{Spec}\left(S_{(f)}\right)$ to $D_{+}(f)$.

Then as $f$ varies, these structures of locally ringed spaces are compatible on the intersections of the various $D_{+}(f)$ 's and hence define the structure of a locally ringed space on $\operatorname{Proj}(S)$ which becomes a scheme and is denoted by $\mathbb{P}_{\mathbb{C}}^{n}$. The underlying set of closed points of this scheme can be canonically identified with complex projective $n$-dimensional space $P_{\mathbb{C}}^{n}$ and hence we say that $\mathbb{P}_{\mathbb{C}}^{n}$ is the scheme (structure) associated to $P_{\mathbb{C}}^{n}$.

Just like $P_{\mathbb{C}}^{n}$ is covered by $(n+1)$ open subsets (each open set corresponding to nonvanishing of a homogeneous coordinate $x_{i}$ ) isomorphic to $\mathbb{C}^{n}$, the scheme $\mathbb{P}_{\mathbb{C}}^{n}$ is also covered by the corresponding $(n+1)$ affine open subschemes each isomorphic to the scheme $\mathbb{A}_{\mathbb{C}}^{n}$ (cf. (A.4.3)). The canonical structure morphism $\mathbb{P}_{\mathbb{C}}^{n} \longrightarrow \operatorname{Spec}(\mathbb{C})$ is the fundamental example of a proper morphism.

A scheme $X$ is said to be projective over $\mathbb{C}$ if there exists a closed immersion of $X$ into the scheme-theoretic projective space $\mathbb{P}_{\mathbb{C}}^{k}$ over $\mathbb{C}$ for some nonnegative integer $k$. If $X$ is projective over $\mathbb{C}$, then its structure morphism is proper.

\section{A.6.8 Smooth Schemes of Finite Type over $\mathbb{C}$}

A scheme $X$ of finite type over $\mathbb{C}$ is said to be smooth at a point $x \in X$ if the local ring $\mathcal{O}_{X, x}$ is a regular local ring. If $X$ is smooth at each of its points, then it is said to be smooth. The words regular and nonsingular are also often used instead of "smooth".

\section{A.6.9 Quasi-coherent and Coherent Algebraic Sheaves}

Let $X=\operatorname{Spec}(A)$ be an affine scheme and $M$ an $A$-module. For $f \in A$, we have the open subset $D(f)$ to which we associate the localization $M_{f}$ of $M$ by $f$ which is canonically a module over $A_{f}$. Since the open subsets of the form $D(f)$ form a base for the Zariski topology on $X$ (see (A.4.3)), 
this association is sufficient to define a unique sheaf of $\mathcal{O}_{X}$-modules on $X$ (cf. (A.3)) which is denoted $\widetilde{M}$ and is called the sheaf of $\mathcal{O}_{X}$-modules on $X$ associated to $M$ and further satisfies the condition that its module of sections over $D(f)$ is isomorphic to $M_{f}$, for each $f \in A$.

The association $M \mapsto \widetilde{M}$ is an exact, fully faithful covariant functor from the category of $A$-modules to the category of $\mathcal{O}_{X}$-modules. For the notion of exactness, see (A.8).

For an arbitrary scheme $X$, an $\mathcal{O}_{X}$-module is said to be quasi-coherent if its restriction to any open affine subscheme is the sheaf associated to a module over the ring defining that affine subscheme in the sense of the first paragraph above. Further, if this happens and the modules concerned are finitely generated, then we call the quasi-coherent sheaf as coherent. All structure sheaves of schemes are coherent.

\section{A.7 Some Definitions from the Theory of Complex Analytic Spaces}

A.7.1 Definitions. Let $\left(X, \mathcal{O}_{X}\right)$ be a complex analytic space as defined in (A.5.4). We say that $\left(X, \mathcal{O}_{X}\right)$ is

1. Hausdorff if the underlying topological space $X$ is Hausdorff;

2. reduced if for each open set $U \subseteq X$, the $\operatorname{ring} \mathcal{O}_{X}(U)$ is reduced i.e., has no nilpotent elements or equivalently if for each point $x \in X$, the local ring $\mathcal{O}_{X, x}$ is reduced;

3. connected if $X$ is connected;

4. irreducible if $X$ is not the union of two distinct nontrivial analytic proper subsets;

5. smooth (or a manifold) if for each point $x \in X$ there exists an open neighborhood restricted to which the locally ringed space structure is isomorphic to the complex manifold structure (see (A.5.2)) of an open subset of $\mathbb{C}^{n}$ for a suitable nonnegative integer $n$;

6. compact if $X$ is compact.

\section{A.7.2 Proper Morphisms}

A morphism of complex analytic spaces is said to be proper if the underlying topological spaces of both are locally compact and the preimage of any compact subset of the target topological space is a compact subset of the source topological space. 


\section{A.7.3 Fiber Products}

Fiber products exist in the category of complex analytic spaces and the definition of a fiber product is analogous to the one for the category of schemes made in (A.6.5) above.

A.7.4 Remark. Many of the notions defined in (A.6) for the category of schemes (like the notions of fiber product as mentioned above, closed and open immersions etc.,) can also be defined for the category of complex analytic spaces. However, we will conclude this section with the definition of a coherent analytic sheaf.

\section{A.7.5 Coherent Analytic Sheaves}

The notion of a coherent analytic sheaf on a complex analytic space corresponds to the notion of a coherent algebraic sheaf on a scheme as defined in (A.6.9) above. The following definition can also be used in the context of scheme theory to obtain an equivalent definition of a coherent algebraic sheaf over a scheme.

Let $\mathcal{A}_{X}$ be a sheaf of rings on a topological space $X$. Let $\mathcal{F}$ be an $\mathcal{A}_{X^{-}}$ module (see (A.3)). Then $\mathcal{F}$ is said to be of finite type if for each $x \in X$ there exists an open neighborhood $U$ restricted to which $\mathcal{F}$ is a quotient of a finite direct sum of sheaves of the form $\left.\mathcal{A}_{X}\right|_{U}$ (recall (A.1.1)).

A sheaf $\mathcal{F}$ of $\mathcal{A}_{X}$-modules is said to be of relation finite type if for each $x \in X$ there exists an open neighborhood $U$ such that every homomorphism of any finite direct sum of sheaves of the form $\left.\mathcal{A}_{X}\right|_{U}$ into $\left.\mathcal{F}\right|_{U}$ has kernel of finite type.

Now let $\left(X, \mathcal{O}_{X}\right)$ be a complex analytic space. By a coherent analytic sheaf we mean a sheaf of $\mathcal{O}_{X}$-modules which is of finite type and also of relation finite type. $\mathcal{O}_{X}$ is itself a coherent analytic sheaf.

A coherent analytic subsheaf $\mathcal{I}$ of $\mathcal{O}_{X}$ is called a coherent analytic ideal sheaf. The set $Y$ of common zeros of sections of $\mathcal{I}$ locally is an analytic subset of $X$ and the locally ringed space $\left(Y, \mathcal{O}_{Y}\right)$ where $\mathcal{O}_{Y}:=\left.\left(\mathcal{O}_{X} / \mathcal{I}\right)\right|_{Y}$ is also a complex analytic space (cf. (A.4.4) and (A.6.4)).

\section{A.8 Sheaf Cohomology}

Let $X$ be a topological space. Let $\mathcal{A B}$ denote the category of abelian groups and Sheaves $(X, \mathcal{A B})$ denote the category of sheaves of abelian groups on $X$. In the category $\mathcal{A B}$, the following properties are obvious:

1. for any two objects $A, B \in \mathcal{A B}, \operatorname{HOM}_{\mathcal{A B}}(A, B)$ which denotes the set of morphisms from $A$ to $B$, is an abelian group; 
2. the composition of morphisms is $\mathbb{Z}$-bilinear i.e., the map

$$
\begin{aligned}
& \operatorname{HOM}_{\mathcal{A B}}(A, B) \times \operatorname{HOM}_{\mathcal{A B}}(B, C) \\
& \quad \longrightarrow \operatorname{HOM}_{\mathcal{A B}}(A, C) \text { given by }(f, g) \mapsto g \circ f
\end{aligned}
$$

satisfies $g \circ\left(f_{1}+f_{2}\right)=g \circ f_{1}+g \circ f_{2}$ and $\left(g_{1}+g_{2}\right) \circ f=g_{1} \circ f+g_{2} \circ f$;

3. finite direct sums exist in $\mathcal{A B}$;

4. every morphism in $\mathcal{A B}$ has a kernel and a cokernel;

5. every monomorphism in $\mathcal{A B}$ is the kernel of its cokernel;

6. every epimorphism in $\mathcal{A B}$ is the cokernel of its kernel;

7. every morphism in $\mathcal{A B}$ can be factored into an epimorphism followed by a monomorphism.

These properties make $\mathcal{A B}$ into what is called an abelian category. It turns out that the above properties on $\mathcal{A B}$ can be transported by using arrow-theoretic definitions in an obvious manner to $\operatorname{Sheaves}(X, \mathcal{A B})$ which also becomes an abelian category.

Next we define the global sections functor

$$
\Gamma(X, \cdot): \text { Sheaves }(X, \mathcal{A B}) \longrightarrow \mathcal{A B} \text { by } \mathcal{F} \mapsto \Gamma(X, \mathcal{F}):=\mathcal{F}(X)
$$

This functor is covariant, left-exact and additive.

Additivity means that for $\mathcal{F}, \mathcal{G} \in \operatorname{Sheaves}(X, \mathcal{A B})$ the induced map

$$
\operatorname{HOM}_{\text {Sheaves }(\mathbf{X}, \mathcal{A B})}(\mathcal{F}, \mathcal{G}) \longrightarrow \operatorname{HOM}_{\mathcal{A B}}(\Gamma(X, \mathcal{F}), \Gamma(X, \mathcal{G}))
$$

is a homomorphism of abelian groups. A sequence of objects and morphisms in an abelian category is said to be exact at an object of the sequence if the image of the incoming map to that object equals the kernel of the outgoing map from that object. A sequence of objects and morphisms in an abelian category is said to be exact if it is exact at each entry (i.e., object) of the sequence. Now left-exactness of the global sections functor means that for a short exact sequence in $\operatorname{Sheaves}(X, \mathcal{A B})$ i.e., an exact sequence of the form

$$
0 \longrightarrow \mathcal{F} \longrightarrow \mathcal{G} \longrightarrow \mathcal{H} \longrightarrow 0
$$

the following corresponding sequence obtained by applying the global sections functor

$$
0 \longrightarrow \Gamma(X, \mathcal{F}) \longrightarrow \Gamma(X, \mathcal{G}) \longrightarrow \Gamma(X, \mathcal{H})
$$

in the category $\mathcal{A B}$ is also exact. Exactness of the global sections functor would mean that we may append $\longrightarrow 0$ to the right of the above sequence to obtain yet another exact sequence; in other words, exact functors transform exact sequences into exact sequences. 
Let us fix an element $\mathcal{F} \in \operatorname{Sheaves}(X, \mathcal{A B})$. We can inductively construct an exact sequence

$$
0 \longrightarrow \mathcal{F} \stackrel{e}{\longrightarrow} \mathcal{I}^{0} \stackrel{d^{0}}{\longrightarrow} \mathcal{I}^{1} \stackrel{d^{1}}{\longrightarrow} \mathcal{I}^{2} \longrightarrow \cdots
$$

of sheaves of abelian groups on $X$, where the $\mathcal{I}^{k}(k \geq 0)$ are all injective objects i.e., the functor $\mathcal{G} \mapsto \operatorname{HOM}_{\text {Sheaves }(X, \mathcal{A B})}\left(\mathcal{G}, \mathcal{I}^{k}\right)$ carries exact sequences into exact sequences, for each $k \geq 0$.

The truncated exact sequence $\mathcal{I}^{0} \stackrel{d^{0}}{\longrightarrow} \mathcal{I}^{1} \stackrel{d^{1}}{\longrightarrow} \mathcal{I}^{2} \stackrel{d^{2}}{\longrightarrow} \mathcal{I}^{3} \longrightarrow \cdots$ is called an injective resolution of $\mathcal{F}$. If we apply the global sections functor to the sequence $(\dagger)$ above, then we get a left-exact sequence

$$
0 \longrightarrow \Gamma(X, \mathcal{F}) \stackrel{E}{\longrightarrow} \Gamma\left(X, \mathcal{I}^{0}\right) \stackrel{D^{0}}{\longrightarrow} \Gamma\left(X, \mathcal{I}^{1}\right) \stackrel{D^{1}}{\longrightarrow} \Gamma\left(X, \mathcal{I}^{2}\right) \longrightarrow \cdots
$$

of abelian groups which may not be exact, but is still a complex in the sense that $D^{i+1} \circ D^{i}=0$ for every $i \geq 0$. We define the $i$-th sheaf cohomology group of $X$ with respect to the sheaf $\mathcal{F}$ denoted $\mathrm{H}^{i}(X, \mathcal{F})$, to be the quotient (Kernel $\left(D^{i}\right)$ /Image $\left.\left(D^{i-1}\right)\right)$ for each nonnegative integer $i$, where we let $D^{-1}$ denote the zero map from the trival abelian group (denoted by 0 in sequences) to the abelian group of global sections of $\mathcal{I}^{0}$. It is clear that the zeroth sheaf cohomology group of $X$ with values in $\mathcal{F}$ is isomorphic to the global sections of $\mathcal{F}$.

The $i$-th sheaf cohomology group measures the extent to which exactness is lost at the $i$-th entry of the injective resolution of the sheaf after applying the global sections functor.

Further, given a short exact sequence of sheaves of abelian groups on $X$

$$
0 \longrightarrow \mathcal{F} \longrightarrow \mathcal{G} \longrightarrow \mathcal{H} \longrightarrow 0
$$

we get a long exact sequence of cohomology groups in $\mathcal{A B}$ written as

$$
\cdots \longrightarrow \mathrm{H}^{i}(X, \mathcal{F}) \longrightarrow \mathrm{H}^{i}(X, \mathcal{G}) \longrightarrow \mathrm{H}^{i}(X, \mathcal{H}) \stackrel{\delta^{i}}{\longrightarrow} \mathrm{H}^{i+1}(X, \mathcal{F}) \longrightarrow \cdots
$$

where the $\delta^{i}$ are called connecting homomorphisms.

By a morphism of sequences (of objects and morphisms) in an abelian category, we mean a collection of morphisms, one for each entry, from the object corresponding to the entry of the first sequence, to the corresponding object of the second sequence, such that all these morphisms together with morphisms of the individual sequences form a commutative diagram i.e., one in which given any two objects, a morphism from one to the other gotten by composing any sequence of morphisms in the diagram is independent of the choice of the composing morphisms. 
Now given a morphism of short exact sequences of sheaves of abelian groups on $X$, there is automatically a morphism of the corresponding long exact sequences of cohomology groups. Because of these properties, the collection of functors $\left\{\mathrm{H}^{i}(X,-) ; i \geq 0\right\}$ is called a covariant delta-functor from the abelian category of sheaves of abelian groups on $X$ to the abelian category of abelian groups. By a theorem of A.Grothendieck (see his exposition in the Tôhoku Math. Journal cited in the References at the end) this deltafunctor is uniquely defined up to a unique isomorphism of delta-functors and hence is called a universal delta-functor.

\section{A.9 Čech Cohomology}

Let $X$ be a topological space, $\mathcal{U}:=\left\{U_{i} ; i \in I\right\}$ an open covering of $X$ and $\mathcal{F}$ a sheaf of abelian groups on $X$. Fix a well-ordering of the indexing set $I$. Let $U_{i_{0} i_{1} \cdots i_{p}}$ denote the intersection $U_{i_{0}} \cap U_{i_{1}} \cap \cdots \cap U_{i_{p}}$ for any finite subset $\left\{i_{0}, \ldots, i_{p}\right\} \subset I$.

For each integer $p \geq 0$, define the following abelian group:

$$
C^{p}(\mathcal{U}, \mathcal{F})=\Pi_{i_{0}<\cdots<i_{p}} \mathcal{F}\left(U_{i_{0} \cdots i_{p}}\right)
$$

where $\Pi$ denotes the operation of taking a direct product of abelian groups. Thus an element $\alpha \in C^{p}(\mathcal{U}, \mathcal{F})$ is a collection

$$
\alpha=\left\{\alpha_{i_{0} \cdots i_{p}} ; i_{0}<\cdots<i_{p}\right\}
$$

where $\alpha_{i_{0} \cdots i_{p}} \in \mathcal{F}\left(U_{i_{0} \cdots i_{p}}\right)$ for each $(p+1)$-tuple $\left(i_{0}, \ldots, i_{p}\right)$ of elements of $I$ with $i_{0}<\cdots<i_{p}$.

For each integer $p \geq 0$, define the following homomorphism of abelian groups:

$$
d^{p}: C^{p}(\mathcal{U}, \mathcal{F}) \longrightarrow C^{p+1}(\mathcal{U}, \mathcal{F}), \alpha \mapsto d^{p} \alpha,
$$

where for $\alpha=\left\{\alpha_{i_{0} \cdots i_{p}} ; i_{0}<\cdots<i_{p}\right\}$ as above, we define $d^{p} \alpha$ as follows:

$$
d^{p} \alpha=\beta=\left\{\beta_{i_{0} \cdots i_{p+1}} ; i_{0}<\cdots<i_{p+1}\right\}, \quad \beta_{i_{0} \cdots i_{p+1}} \in \mathcal{F}\left(U_{i_{0} \cdots i_{p+1}}\right),
$$

where $\beta_{i_{0} \cdots i_{p+1}}$ is the sum of the restrictions to $\mathcal{F}\left(U_{i_{0} \cdots i_{p+1}}\right)$ of the following $(p+2)$ local sections of $\mathcal{F}$ :

$$
\alpha_{i_{1} \cdots i_{p+1}} ;(-1)^{k} \alpha_{i_{0} \cdots i_{k-1} i_{k+1} \cdots i_{p+1}},(1 \leq k \leq p) ;(-1)^{p+1} \alpha_{i_{0} \cdots i_{p}} .
$$

It is easy to see that $d^{p+1} \circ d^{p}=0$ for each $p \geq 0$, so that Image $\left(d^{p-1}\right) \subset$ Kernel $\left(d^{p}\right)$ for each $p \geq 1$. Therefore the following sequence is a complex of abelian groups and group homomorphisms (cf. (A.8)):

$$
C^{*}(\mathcal{U}, \mathcal{F}): 0 \stackrel{d^{-1}}{\longrightarrow} C^{0}(\mathcal{U}, \mathcal{F}) \stackrel{d^{0}}{\longrightarrow} C^{1}(\mathcal{U}, \mathcal{F}) \stackrel{d^{1}}{\longrightarrow} C^{2}(\mathcal{U}, \mathcal{F}) \stackrel{d^{2}}{\longrightarrow} \cdots
$$


We define the abelian group $\check{\mathrm{H}}^{p}(\mathcal{U}, \mathcal{F})$, for each integer $p \geq 0$, called the $p$-th Čech cohomology group of $X$ with values in the sheaf $\mathcal{F}$ relative to the covering $\mathcal{U}$ as the following quotient:

$$
\check{\mathrm{H}}^{p}(\mathcal{U}, \mathcal{F}):=\operatorname{Kernel}\left(d^{p}\right) / \operatorname{Image}\left(d^{p-1}\right) .
$$

Each map $d^{p}$ is called a coboundary map or a differential map for the complex $C^{*}(\mathcal{U}, \mathcal{F})$. We often denote the kernel of $d^{p}$ by $Z^{p}(\mathcal{U}, \mathcal{F})$ and call its elements as $p$-cocyles. Further, the elements of $C^{p}(\mathcal{U}, \mathcal{F})$ are called $p$-cochains and those of the image of $d^{p-1}$ are called $p$-coboundaries.

Now we further assume that $X$ is a compact complex manifold and that each open set $U_{i}$ is biholomorphic to a polydisc in $\mathbb{C}^{n}$, where $n$ is the dimension of $X$ as a complex manifold, and further that all the higher sheaf cohomology groups of any finite intersection of these open sets with values in the sheaf $\mathcal{F}$ restricted to this intersection, vanish (this condition is satisfied, for example, if one is able to find a cover such that any finite intersection of open sets of this cover is biholomorphic to a polydisc). Such a cover does exist for any compact complex manifold, and is called a Leray Cover.

In all computations involving Čech cohomology of a compact complex manifold in this book, we will work only with a Leray cover, though we may not state this explicitly.

We next define the $p$-th Čech cohomology group of $X$ with values in the sheaf $\mathcal{F}$ to be the abelian group $\check{\mathrm{H}}^{p}(\mathcal{U}, \mathcal{F})$ defined above; further, this group is found to be uniquely determined irrespective of the choice of the cover $\mathcal{U}$, provided it is a Leray cover as required above, and hence we denote this group as $\check{\mathrm{H}}^{p}(X, \mathcal{F})$.

It can be proved that the collection of functors $\left\{\check{\mathrm{H}}^{i}(X, \mathcal{F}) ; i \geq 0\right\}$ is a covariant delta functor (see (A.8)). Further, it is nontrivial to prove that this collection of functors is a universal delta functor isomorphic to the collection of sheaf cohomology functors $\left\{\mathrm{H}^{i}(X, \mathcal{F}) ; i \geq 0\right\}$ (which is also a universal delta functor as stated in (A.8)).

\section{A.10 Algebraizability of Complex Analytic Spaces}

\section{A.10.1 The Complex Analytic Space Associated to a Scheme of Finite Type Over $\mathbb{C}$}

Let $A$ be a finitely generated algebra over $\mathbb{C}$. Thus $A$ is isomorphic to the quotient of the polynomial ring $\mathbb{C}\left[z_{1}, \ldots, z_{n}\right]$ in $n$ variables over $\mathbb{C}$ (for some integer $n \geq 0)$ by a finitely generated ideal $I=\left(f_{1}, \ldots, f_{q}\right)$. Then we get 
the affine scheme $X=\operatorname{Spec}(A)$ which is a closed embedded subscheme of $\mathbb{A}_{\mathbb{C}}^{n}($ see $(\mathrm{A} .4 .3)$ and (A.6.3)). $X$ is trivially a scheme of finite type over $\mathbb{C}$. Now $f_{1}, \ldots, f_{q}$ are polynomial functions on $\mathbb{C}^{n}$, hence holomorphic, so their set of common zeros is a complex analytic subset of $\mathbb{C}^{n}$ (see (A.4.4) and (A.7.5)) which is a complex analytic closed subspace denoted by $X_{H O L}$ and is called the complex analytic space associated to $X=\operatorname{Spec}(A)$.

Next, if $X$ is a scheme of finite type over $\mathbb{C}$ (see (A.6.3)), then $X$ can be covered by a finite number of open affine subschemes of the form $\operatorname{Spec}(A)$ mentioned in the previous paragraph, which glue together to give $X$. Therefore the associated complex analytic spaces $(\mathbf{S p e c}(A))_{H O L}$ (as defined in the previous paragraph) also glue together to give a complex analytic space which is called the complex analytic space associated to $X$ and is denoted by $X_{H O L}$.

The association $X \mapsto X_{H O L}$ from the category of schemes of finite type over $\mathbb{C}$ to the category of complex analytic spaces is a covariant functor.

\section{A.10.2 The Coherent Analytic Sheaf Associated to a Coherent Algebraic Sheaf}

Let $\mathcal{F}$ be a coherent algebraic sheaf on a scheme $X$ of finite type over $\mathbb{C}($ see (A.6.9)). For any open affine subscheme $U=\operatorname{Spec}(A)$ of $X$, by definition the sheaf $\left.\mathcal{F}\right|_{U}$ (recall (A.1.1)) is the sheaf $\widetilde{M}$ associated to a finitely generated module $M$ over $A$. Therefore we have an exact sequence of sheaves of $\mathcal{O}_{U}$-modules

$$
\left.\mathcal{O}_{U}^{m} \stackrel{\phi}{\longrightarrow} \mathcal{O}_{U}^{n} \longrightarrow \mathcal{F}\right|_{U} \longrightarrow 0
$$

equivalent to an exact sequence of $A$-modules $A^{m} \stackrel{\tilde{\phi}}{\longrightarrow} A^{n} \longrightarrow M \longrightarrow 0$ where $A^{m}$ denotes the free module of rank $m$ over $A$ (see (A.6.9) and $($ A.7.5)).

The usual topology (i.e., in the complex analytic sense) is finer than the Zariski topology, so $U_{H O L}$ is open in $X_{H O L}$. Now $\phi$ is defined by the matrix of the map $\tilde{\phi}$ with entries in $A$. These entries can be thought of as sections of $\mathcal{O}_{U}$ over $U$, i.e., as polynomial functions over $U$. Hence they can be also thought of as holomorphic functions over $U_{H O L}$, and thus give rise to sections of the free coherent analytic sheaf $\mathcal{O}_{U_{H O L}}$ of germs of holomorphic functions on $U_{H O L}$. Thus we get a map $\phi_{H O L}$ of coherent analytic sheaves

$$
\mathcal{O}_{U_{H O L}}^{m} \stackrel{\phi_{H O L}}{\longrightarrow} \mathcal{O}_{U_{H O L}}^{n}
$$

and we define $\left.\mathcal{F}_{H O L}\right|_{U}$ to be the cokernel sheaf (i.e., sheaf associated to the presheaf cokernel) of $\phi_{H O L}$. Then as $U$ varies in $X$, the coherent analytic 
sheaves $\left.\mathcal{F}_{H O L}\right|_{U}$ patch up together (in a compatible way - since the sheaves $\left.\mathcal{F}\right|_{U}$ patch up to give $\mathcal{F}$ ) to give a coherent analytic sheaf (recall that the notion of coherence is local) which we denote by $\mathcal{F}_{H O L}$ and define to be the coherent analytic sheaf associated to the coherent algebraic sheaf $\mathcal{F}$.

The association $\mathcal{F} \mapsto \mathcal{F}_{H O L}$ from the category of coherent algebraic sheaves over $X$ to the category of coherent analytic sheaves over $X_{H O L}$ is a covariant functor.

\section{A.10.3 Cohomologies of a Coherent Algebraic Sheaf and its Associated Coherent Analytic Sheaf}

There is an obvious continuous map of topological spaces $\phi: X_{H O L} \longrightarrow X$ sending points of $X_{H O L}$ bijectively onto the set of closed points of $X$. Next, there is a natural morphism of sheaves of rings on $X_{H O L}$, induced by $\phi$, from the inverse image sheaf $\phi^{-1} \mathcal{O}_{X}$ to $\mathcal{O}_{X_{H O L}}$. Under the canonical bijection (cf. (A.3))

$$
\operatorname{HOM}_{S h\left(X_{H O L}\right)}\left(\phi^{-1} \mathcal{O}_{X}, \mathcal{O}_{X_{H O L}}\right) \cong \operatorname{HOM}_{S h(X)}\left(\mathcal{O}_{X}, \phi_{*} \mathcal{O}_{X_{H O L}}\right),
$$

this morphism corresponds to a morphism of sheaves of rings on $X$ which we denote by $\phi^{\#}: \mathcal{O}_{X} \longrightarrow \phi_{*} \mathcal{O}_{X_{H O L}}$ where $\phi_{*} \mathcal{O}_{X_{H O L}}$ is the pushforward sheaf. Then it can be checked that

$$
\left(\phi, \phi^{\#}\right): X_{H O L} \longrightarrow X
$$

is a morphism of locally ringed spaces. Further, for any coherent sheaf $\mathcal{F}$ on $X, \phi^{*} \mathcal{F}$ is isomorphic to $\mathcal{F}_{H O L}$. Then we get natural maps of the sheaf cohomology groups

$$
\alpha_{i}: \mathrm{H}^{i}(X, \mathcal{F}) \longrightarrow \mathrm{H}^{i}\left(X_{H O L}, \mathcal{F}_{H O L}\right),(i \geq 0) .
$$

\section{A.10.4 Properties of the Associated Complex Analytic Space}

We have defined in (A.10.1) the functor $X \mapsto X_{H O L}$ from the category of schemes of finite type over $\mathbb{C}$ to the category of complex analytic spaces. We state the following theorem that compares properties of $X$ with those of $X_{H O L}$.

A.10.4.1 THEOREM. Let $X$ be a scheme of finite type over $\mathbb{C}$ and $X_{H O L}$ its associated complex analytic space. Then:

1. $X$ is separated over $\mathbb{C}$ if and only if $X_{H O L}$ is Hausdorff; 
2. $X$ is connected in the Zariski topology if and only if $X_{H O L}$ is connected in the usual topology of complex analytic spaces;

3. $X$ is reduced if and only if $X_{H O L}$ is reduced;

4. $X$ is smooth over $\mathbb{C}$ if and only if $X_{H O L}$ is a complex manifold;

5. A morphism $f: X \longrightarrow Y$ (where $Y$ is also a scheme of finite type over $\mathbb{C})$ is proper if and only if $f_{\mathrm{HOL}}: X_{\mathrm{HOL}} \longrightarrow Y_{\mathrm{HOL}}$ is proper. In particular, $X$ is proper over $\operatorname{Spec}(\mathbb{C})$ if and only if $X_{H O L}$ is a compact analytic space.

\section{A.10.5 The GAGA Correspondence}

The following fundamental results on algebraizability of complex analytic subspaces of complex projective spaces are due to J-P.Serre (see his GAGA paper listed under the References at the end). An earlier result of Chow [3] also follows as a corollary.

\section{A.10.5.1 THEOREM (GAGA Correspondence).}

1. Let $\mathcal{X}$ be a complex analytic closed subspace of some complex projective space $P_{\mathbb{C}}^{n}$. Then there exists a projective scheme $X$ over $\mathbb{C}$ such that $X_{H O L} \cong \mathcal{X}$ as complex analytic spaces. In other words, we say $\mathcal{X}$ is algebraic or algebraizable.

2. If $X$ and $X^{\prime}$ are two projective schemes over $\mathbb{C}$ such that their associated complex analytic spaces are isomorphic, then they are themselves isomorphic as schemes. In this sense, the scheme $X$ of (1) above is unique.

3. Let $X$ be a projective scheme over $\mathbb{C}$. Then then functor $\mathcal{F} \mapsto \mathcal{F}_{H O L}$ from the category of coherent algebraic sheaves over $X$ to the category of coherent analytic sheaves over $X_{H O L}$ is an equivalence of categories. In particular we have:

(a) given a projective scheme $X$ over $\mathbb{C}$ and a coherent analytic sheaf $\tilde{\mathcal{F}}$ on $X_{H O L}$, there exists a coherent algebraic sheaf $\mathcal{F}$ on $X$ such that $\mathcal{F}_{H O L}$ is isomorphic to $\tilde{\mathcal{F}}$ on $X_{H O L}$;

(b) given a projective scheme $X$ over $\mathbb{C}$ and two coherent algebraic sheaves $\mathcal{E}, \mathcal{F}$ on $X$ such that their associated coherent analytic sheaves are isomorphic on $X_{H O L}$, then they are themselves isomorphic as coherent algebraic sheaves on $X$. In this sense, the coherent sheaf $\mathcal{F}$ of (a) above is unique.

4. For each integer $i \geq 0$, and for each coherent algebraic sheaf $\mathcal{F}$ on $X$, the natural map of cohomology groups

$$
\alpha_{i}: H^{i}(X, \mathcal{F}) \longrightarrow H^{i}\left(X_{H O L}, \mathcal{F}_{H O L}\right)(i \geq 0),
$$

is an isomorphism. 
A.10.5.2 COROLLARY (CHOW'S THEOREM). A compact complex analytic subspace $\mathcal{X}$ of the complex manifold $P_{\mathbb{C}}^{n}$ is canonically algebraizable in the sense that it is the complex analytic space associated to a closed subscheme $X$ of the scheme associated to this projective space (in particular, $X$ is proper over $\mathbb{C}$ ).

A.10.5.3 Note. It can be shown as an application of Zariski's Main Theorem that if a compact complex analytic space is algebraizable, then any algebraic structure on it is uniquely determined up to a unique isomorphism. This assertion is false if we do not assume compactness. 



\section{References}

Note: The following references are ordered in the sequence in which they are quoted from the beginning chapter of this book.

[1] H.M.Farkas, I.Kra Riemann Surfaces Second Edition Graduate Texts in Mathematics GTM 71 Springer-Verlag, New York 1991

[2] O.Forster Lectures on Riemann Surfaces First Edition Graduate Texts in Mathematics GTM 81 Springer-Verlag, New York 1981

[3] W.L.Chow On Compact Complex Analytic Varieties Amer. J. of Math. 711949 pp. 893-914 (errata) Amer, J. of Math. 72 p. 624

[4] L.V.Ahlfors Complex Analysis International Student Edition International Series in Pure and Applied Mathematics Mc Graw-Hill, Singapore $\mathbf{1 9 7 0}$

[5] K.Kodaira Complex Manifolds and Deformation of Complex Structures First Edition A Series of Comprehensive Studies in Math. 283 Springer-Verlag, New York 1986

[6] K.Kodaira, D.C.Spencer On Deformations of Complex Analytic Structures, I-II, III Annals of Math. 671958 pp. 328-466 Annals of Math. $71 \mathbf{1 9 6 0}$ pp. 43-76

[7] C.S.Seshadri Theory of Moduli In Algebraic Geometry, Arcata 1974 Proceedings of Symposia in Pure Math. Ed. R. HARTshorne American Mathematical Society Vol. 291975 pp. 263-304

[8] C.L.Siegel Discontinuous Groups Annals of Math. 441946 pp. $674-689$

[9] D.Mumford Abelian Varieties Second Edition TIFR Studies in Mathematics 5 Oxford University Press, Bombay 1974 
[10] A.Fröhlicher, A.Nijenhuis A Theorem on Stability of Complex Structures Proc. Nat. Acad. Sci., USA 431957 pp. 239-241

[11] H.Grauert Ein Theorem der Analytischen Garbentheorie und die Modulräume Komplexer Strukturen Publ. Math. IHES 5 1960 pp. 233-291

[12] W.Fischer, H.Grauert Lokal-trivial Familen Kompakter Komplexen Mannigfaltigkeiten Nachr. Akad. Wiss. Gottingen II. Math. -Phys. K1 1965 pp. 88-94

[13] K.Kodaina, D.C.Spencer A Theorem of Completeness for Complex Analytic Fiber Spaces Acta Math. 1001958 pp. 281-294

[14] M.Kuranishi On the Locally Complete Families of Complex Analytic Structures Annals of Math. 751962 pp. 536-577

[15] H.Grauert Der Satz von Kuranishi für Kompakter Komplexe Räume Invent. Math. 251974 pp. 107-142

[16] B.Riemann Theorie der Abel'schen Functionen J. Reine Angew. Math. (Crelle's Journal) 541857 pp. 115-155

[17] M.S.Narasimhan, R.R.Simha, R.NARAsimhan, C.S.Seshadri Riemann Surfaces TIFR Mathematical Pamphlets 1 School of Mathematics, TIFR, Bombay 1963

[18] M.S.Narasimhan Vector Bundles on Compact Riemann Surfaces Complex Analysis and its Applications Vol. III Intl. atomic Energy Agency, Vienna 1976 pp. 63-88

[19] O.Teichmüller Extremale Quaskkonforme Abbildungen und Quadratische Differentiale Abh. Preuss. Akad. der Wiss. Math. naturw. Klasse 221939 pp. 1-97

[20] M.Schlessinger Functors of Artin Rings Trans. Amer. Math. Soc. Vol. 1301968 pp. 208-222

[21] H.Lange, C.Birkenhake Complex Abelian Varieties A Series of Comprehensive Studies in Mathematics 302 Springer-Verlag 1992

[22] S. LAng Algebra Third Edition Addison-Wesley Publishing Co., USA 1993 
[23] D.Mumford, K.Suominen Introduction to the Theory of Moduli Lecture Notes Nordic Summer School in Math. Oslo Ed. F.OorT Walters-Noordhoff 1711970 pp. 171-222

[24] D.Mumford Picard Groups of Moduli Problems Arithmetical Algebraic Geometry Harper \& Row New York 1965 p. 58

[25] R.Hartshorne Algebraic Geometry First Edition Graduate Texts in Mathematics GTM 52 Springer-Verlag, New York 1977

[26] H.Grauert, R.Remmert Coherent Analytic Sheaves First Edition A Series of Comprehensive Studies in Mathematics 265 SpringerVerlag, Berlin Heidelberg 1984

[27] A.Grothendieck Sur Quelques Points d'Algébre Homologique Tôhoku Math. J. 91957 pp. 119-221, chap. II, 2.2.1

[28] J.-P.Serre Géométrie Algébrique et Géométrie Analytique (GAGA) Ann. Inst. Fourier 61956 pp. 1-42

[29] L.Bers Uniformization and Moduli Contributions to Function Theory, Bombay Colloquim Tata Inst. of Fundamental Research 1960 pp. $41-50$

[30] M.ArTin The Implicit Function Theorem in Algebraic Geometry Proc. of the Bombay Colloq., on Alg. Geometry 1968

[31] D.Mumford, J.Fogarty, F.KIrwan Geometric Invariant Theory Third Enlarged Edition A Series of Modern Surveys in Mathematics 34 Springer-Verlag Berlin Heidelberg 1994

Advanced Texts on Classification Theory, Deformation Theory and Moduli Theory.

[32] K.Ueno Classification Theory of Algebraic Varieties and Compact Complex Spaces Notes written in collaboration with P. Cherenack. Lecture Notes in Mathematics 439 Springer-Verlag, Berlin-New York, 1975

[33] H.Popp Moduli Theory and Classification Theory of Algebraic Varieties Lecture Notes in Mathematics 620 Springer-Verlag, BerlinNew York, 1977

[34] M.Schlichenmaier An Introduction to Riemann Surfaces, Algebraic Curves and Moduli Spaces Lecture Notes in Physics 322 Springer, Berlin 1989 
[35] Y.I.Manin Frobenius Manifolds, Quantum Cohomology, and Moduli Spaces American Mathematical Society Colloquium Publications 47, American Mathematical Society, Providence, RI 1999

[36] Y.Shimizu, K.Ueno Advances in Moduli Theory Translated from the 1999 Japanese original. Translations of Mathematical Monographs 206, Iwanami Series in Modern Mathematics, American Mathematical Society, Providence, RI 2002

[37] S.MukaI, An Introduction to Invariants and Moduli Translated from the 1998 and 2000 Japanese editions by W. M. Oxbury. Cambridge Studies in Advanced Mathematics 81, Cambridge University Press, Cambridge 2003

[38] E.Sernesi Deformations of Algebraic Schemes Grundlehren der Mathematischen Wissenschaften 334, Springer-Verlag, Berlin 2006

[39] E.Arbarello Moduli Spaces of Curves-Five Lectures IMPA Mathematical Publications, 26th Brazilian Mathematics Colloquium $I M P A$, Rio de Janeiro 2007

[40] M.Schlichenmaier An Introduction to Riemann Surfaces, Algebraic Curves and Moduli Spaces With an introduction by Ian McArthur. Second Edition Theoretical and Mathematical Physics Springer, Berlin 2007

[41] R.Hartshorne Deformation Theory Graduate Texts in Mathematics 257 Springer 2009

Advanced Text on Moduli of Curves.

[42] J.HARris, I.Morrison Moduli of Curves Graduate Texts in Mathematics GTM 187 Springer-Verlag, New York 1998

Advanced Text on Moduli of Vector Bundles.

[43] D.Huybrechts, M.Lehn The Geometry of Moduli Spaces of Sheaves Aspects of Mathematics 31 Vieweg 1997 Cambridge University Press reprint 2010 
Moduli Theory is one of those areas of Mathematics that has fascinated minds from classical to modern times. This has been so because it reveals beautiful Geometry naturally hidden in questions involving classification of geometric objects and because of the profound use of the methods of several areas of Mathematics like Algebra, Number Theory, Topology and Analysis to achieve this revelation. A study of Moduli Theory would therefore give senior undergraduate and graduate students an integrated view of Mathematics. The present book is a humble introduction to some aspects of Moduli Theory.

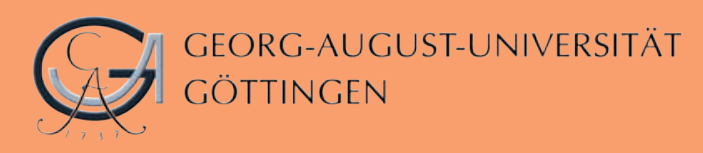

